\title{
Reconstruction of a radioactive source distribution using a tomographic spatial-unfolding method with Compton gamma imager measurements
}

Nathan J. Murtha

A thesis submitted to the Faculty of Graduate and Postdoctoral Affairs in partial fulfilment of the requirements for the degree of

Doctor of Philosophy

in

\author{
Physics \\ Specialization in Medical Physics
}
Ottawa-Carleton Institute for Physics
Department of Physics
Carleton University
Ottawa, Ontario, Canada
2021

(c) 2021 Nathan J. Murtha 


\section{Abstract}

Advanced detection and reconstruction techniques are utilized to characterize the distribution of radioactive materials in radiological safety and security operations. Most radioactive materials can be detected through their gamma-ray emissions. There can arise circumstances in which the measurement of these gamma rays must be made from behind a pre-established perimeter, however. To address this, Natural Resources Canada (NRCan) and the National Research Council of Canada (NRC) have jointly developed novel Compton gamma imaging technology for use in safety and security applications. Compton gamma imaging utilizes the physics of Compton scattering to locate gamma-ray sources, making the technology well-suited to localization at a distance from the suspected source.

A fast tomographic reconstruction method has been developed for use with Compton gamma imaging measurements to provide rapid, in situ source localization. For a more precise offline reconstruction of the data, a spatial unfolding technique has also been developed that fits the observed experimental tomogram with tomographicresponse templates produced by Monte Carlo simulation of spatially-localized sources of radioactivity.

These methods have been applied to two sets of experimental data using measurements acquired with the detector developed by NRCan and the NRC. In one experiment, measurements of a Cs-137 point source were acquired in a laboratory setting. In a second experiment, measurements of a spatially-extended La-140 source were acquired in realistic outdoor operational conditions. The tomographic reconstruction of the sources takes only minutes and demonstrates good localization capability. The spatial-unfolding method shows good localization of experimentally-measured sources but underestimates their activity, while accurately recovering the activity of synthetic sources. The underestimation of experimentally-measured source activities is ascribed to limitations in the simulated representation of the detector response and environmental terrain not included in the simulated world.

The simple tomographic reconstruction method will be useful in guiding radiological safety and security operations in near real-time. The post-process unfolding

method will further be useful in informing follow-on consequence management operations and remediation activities. 


\section{Acknowledgments}

I would like to thank my supervisors, Dr. Laurel Sinclair and Dr. Patrick Saull, for their guidance, their patience, and for providing me with several fantastic opportunities throughout my graduate studies. I have grown as a researcher while working with them, and I will forever be grateful for the lessons learnt. I would also like to thank Dr. Andrew McCann and Dr. Audrey MacLeod, two other core members of the Compton imaging research team, for their support, their encouragement, and for being constantly willing to provide their insight and assistance. Spending these years as part of the "Compton crew" has been one of my most unique and valuable experiences.

My thanks go also to the members of my committee, Dr. Paul Johns and Dr. André Staudte (in addition to Dr. Sinclair and Dr. Saull), for periodically meeting with me during my degree and offering their thoughts and suggestions for my research.

I am thankful to Natural Resources Canada and the National Research Council Canada for hosting me during my time as a graduate student. The groups at each agency were welcoming to me from the beginning, and I feel that it was a valuable experience to be able to work with each of them. I would also like to extend my thanks to the team at Defence Research and Development Canada Suffield Research Centre, without whom the trial presented in chapter 6 would not have been possible.

To Dr. Frédéric Tessier, Dr. Reid Townson, and Dr. Ernesto Mainegra-Hing, a warm thank you for answering my questions about the EGSnrc toolkit. A special thank you to Dr. Tessier for demonstrating endless patience in the face of my many questions about collimated source classes!

Of course, my family supported me greatly throughout my degree. For their advice, patience, and love, my sincere thanks go to my parents and stepparents. My thanks also to the rest of my family, who similarly offered their support and understanding when it was needed.

A final thanks to my fiancée, Brooklyn, whose selfless patience and unwavering support made even the most difficult of times feel (relatively) painless. Your doodles made a difference!

The detector technology presented in this thesis was funded by Defence Research and Development Canada's (DRDC) Chemical, Biological, Radiological-Nuclear and 
Explosives Research and Technology Initiative (CRTI) under project CRTI 07-0193, and through DRDC's Centre for Security Science (CSS) projects CSSP-2014-TA2074, CSSP-2015-CP-2096, and CSSP-2018-TI-2390. The work presented in chapter 6 was supported by CSS project CSSP-2016-TI-2290. Additional funding support was provided from the Natural Sciences and Engineering Research Council of Canada (NSERC), the Ontario Graduate Scholarship (OGS) program, Carleton University, the National Research Council's Metrology Research Centre, and Natural Resources Canada's Canadian Hazards Information Service. 


\section{Statement of contribution}

This thesis gives a summary of my research as a $\mathrm{PhD}$ student at Carleton University. I was supervised by Dr. Laurel Sinclair and Dr. Patrick Saull and worked as part of a team that additionally consisted of Dr. Andrew McCann and Dr. Audrey MacLeod, each of whom contributed to the research presented in this thesis. The coding, data analysis, and writing, where not otherwise indicated below, were performed by me.

Dr. Sinclair provided me with code to extract Global Navigation Satellite System (GNSS) data from a proprietary binary file format. I used this code to extract GNSS information, and used the GNSS information to obtain position and orientation estimates for a detector as it was placed during an experiment.

I inherited EGSnrc Monte Carlo simulation code from Dr. Saull that contained a simulated representation of the detector used for the work presented in this thesis, along with some associated code scripts to process the output. To the simulated representation of the detector, I added a carbon fibre body as well as carbon fibre enclosures for the detector planes. I created new material density correction data for simulations as needed. Additionally, I added functionality to the EGSnrc application code that allows for the emission location of all primary histories to be tracked and used in later analysis, and wrote the code required to process this information. Finally, I wrote code that adjusts the empirical energy smearing applied during analysis of the simulated data so that the smearing better reflects the calibration status of each detector channel as it existed in experimental conditions.

To increase the efficiency of Monte Carlo simulations performed in EGSnrc, I proposed the use of the EGS_CollimatedSource class with a rectangular collimated target shape. Dr. Saull advised the use of a spherical target shape, and subsequent conversations about this approach with Dr. Frédéric Tessier led to a request being made of Dr. Reid Townson to implement a collimation target shape consisting of a circular surface whose normal is always parallel with the initial trajectory of the primary emission direction for a given simulated history; this target shape class now exists in the EGSnrc code as the egs_circle_perpendicular class. Following Dr. Townson's implementation of egs_circle_perpendicular, I conducted very minor testing of the class and suggested corrections for a couple of small bugs in the code.

Dr. Sinclair suggested that the subject of my thesis be on the application of tomographic reconstruction techniques to Compton gamma imaging measurements. Dr. 
Saull suggested that Dr. Sinclair's previous work in spatial unfolding of aerial survey measurements could be applied to tomographic reconstructions made with Compton gamma imaging measurements, and Dr. Sinclair was the first to conceptualize how the method could be translated to tomographic Compton imaging measurements. I took Dr. Sinclair's vision of the unfolding method and formalized it, developing the algorithms and writing the code necessary for the analysis. Using the EGSnrc code that I developed as described above, I created a method to submit fewer simulation jobs to computer clusters while still allowing for generation of the required template data for use in the spatial-unfolding method. I wrote the codes that processed the experimental and simulated data and that subsequently performed tomographic image reconstruction and spatially-unfolded reconstructions. Dr. Sinclair proposed the Monte Carlo regeneration method of statistical uncertainty estimation that is presented for the results of the spatial unfolding analysis, and I proposed the bootstrapping method of statistical uncertainty estimation.

Dr. Saull proposed the point-model fit method presented in this thesis. I developed the parametrization scheme used in modelling the response of the detector to a point source.

Dr. Sinclair and Dr. Saull suggested the use of the MINUIT minimizer for all minimizations carried out in my code. Inspired by this, I instead applied the updated Minuit2 minimizer for all function minimizations required by the spatial-unfolding method and the point-model fit method.

For the experiment presented in chapter 5, measurements of a Cs-137 point source were conducted by Dr. Saull and his University of Waterloo co-op student, Iain Cornick. Mr. Cornick processed the LIDAR data and provided a coordinate system and detector position/orientation estimates that were subsequently used in my analysis. Dr. Saull provided information regarding the experimental trial and the status of the detector during the experiment.

For the experiment presented in chapter 6, the planning and deposition of an extended La-140 source was carried out by Dr. Sinclair and a team of scientists at Defence Research and Development Canada. I chose the measurement positions that were used when collecting experimental data for tomographic reconstruction, and I operated the detector during the measurements.

Prior to the experiment presented in chapter 6, I wrote a "user's manual" that explained how to operate the Compton gamma imaging detector. This manual was later used in subsequent experiments and operational scenarios. 


\section{Published and presented works}

\section{Peer-reviewed papers}

- L.E. Sinclair, A. McCann, P.R.B. Saull, R.L. Mantifel, C.V.O. Ouellet, P.L. Drouin, A.M.L. MacLeod, B. Le Gros, I. Summerell, J.H. Hovgaard, S. Monkhouse, F. Stanescu, G. Jonkmans, N. Murtha. "End-user experience with the SCoTSS Compton imager and directional survey spectrometer", Nuclear Inst. and Methods in Physics Research A, 954 (2020):161683, doi: 10.1016/j.nima.2018.11.142

\section{Submitted papers}

- N. Murtha, L.E. Sinclair, P.R.B. Saull, A. McCann, A.M.L. MacLeod. "Tomographic reconstruction of a spatially-extended source from the perimeter of a restricted-access zone using a SCoTSS Compton gamma imager", Journal of Environmental Radioactivity, 2021.

\section{Papers in preparation}

- N. Murtha, P.R.B. Saull, L.E. Sinclair, A.M.L. MacLeod, A. McCann. "A tomographic spatial-unfolding method for Compton gamma imaging measurements", 2021.

- N. Murtha, P.R.B. Saull, A.M.L. MacLeod, L.E. Sinclair, A. McCann. "Application of Compton gamma imaging tomography to the reconstruction of multiple extended sources", 2021.

\section{Conference proceedings paper}

(* indicates presenting author) 
- N.J. Murtha*, P.R.B. Saull, L.E. Sinclair, R. Mantifel, A. McCann, A.M.L. MacLeod, B. Beckman, B. Fairbrother, C. Ouellet, P.-L. Drouin. "Reconstruction of an Inaccessible, Large-Area Extended Source with a SCoTSS Compton Gamma Imager", 2018 IEEE Nuclear Science Symposium and Medical Imaging Conference Proceedings (NSS/MIC), Nov. 2018, pp. 1-7, doi: 10.1109/NSSMIC.2018.8824407

\section{Conference abstracts}

$\left({ }^{*}\right.$ indicates presenting author)

- L. Sinclair*, A. McCann, P. Saull, N. Murtha, A. MacLeod. "Unfolding Directional Aerial Radiation Survey Maps to enable Extrapolation and Improved Precision". e-Poster, CTBT Science and Technology 2021 Conference, Jun. 2021. https://conferences.ctbto.org/event/7/contributions/ 915/contribution.pdf

- L.E. Sinclair*, P.R.B. Saull, A. McCann, A.M.L. MacLeod, N.J. Murtha, A. El-Jaby, G. Jonkmans. "Gamma Imagers for Nuclear Security and Nuclear Forensics". Oral presentation, International Conference on Nuclear Security 2020, Vienna, Austria, Feb. 2020. https://conferences.iaea.org/event/ 181/contributions/15694/

- N. Murtha, L.E. Sinclair*, P.R.B. Saull, A.M.L. MacLeod, A. McCann. "Gamma imaging for on-site inspection: reconstruction of an extended source in a restricted-access zone". Oral presentation, CTBT Science and Technology 2019 Conference, Vienna, Austria, Jun. 2019. https://conferences.ctbto. org/event/3/contributions/231/

- A. McCann*, L.E. Sinclair, P.R.B. Saull, A.M.L. MacLeod, N. Murtha. "Surround imaging with traditional two-plane and novel $4 \pi$-optimized Compton imagers". Poster presentation, IEEE Nuclear Science Symposium and Medical Imaging Conference, Manchester, UK, Oct. 2019. https://www.eventclass . org/contxt_ieee2019/online-program/session?s=N-05

- A.M.L. MacLeod, A. McCann, N. Murtha*, P.R.B. Saull, L.E. Sinclair. "Applications of Compton gamma imaging to radiation protection and nuclear security". Oral presentation, Canadian Radiation Protection Association Annual Conference, Ottawa, Canada, May 2019. 


\title{
List of abbreviations and symbols
}

\author{
ADC .... Analogue-to-digital converter \\ ARM . . . . Angular resolution measure \\ BV206 . . . . Hägglunds Bandvagn 206 tracked vehicle \\ CsI(Tl) . . . Thallium-doped cesium iodide \\ CTBT . . . C Comprehensive Nuclear-Test-Ban Treaty \\ CZT .... Cadmium Zinc Telluride \\ DRDC . . . Defence Research and Development Canada \\ FNEP . . . . Federal Nuclear Emergency Plan \\ FWHM . . . Full-width at half-maximum \\ GNSS . . . Global navigation satellite system \\ HPGe . . . High-purity germanium \\ ICP . . . . I Iterative closest point \\ ICRU . . . . International Commission on Radiation Units and Measurements \\ LIDAR . . . . Light Detection and Ranging \\ MC ..... Monte Carlo \\ NORM . . . Naturally occurring radioactive materials \\ NRCan . . . Natural Resources Canada \\ NRC . . . . National Research Council \\ SCoTSS . . S Silicon photomultiplier-based Compton Telescope for Safety and \\ Security \\ SiPM . . . S Silicon photomultiplier \\ SPAD ... Single-photon avalanche diode
}


UAV .... . Unmanned aerial vehicle

UGV . . . . Unmanned ground vehicle

UTM .... . Universal Transverse Mercator

A ...... Atomic mass number

$\alpha \ldots \ldots \quad$ Fine structure constant [unitless] or polar angle of Compton cone axis [radians]

$\alpha^{\prime} \ldots \ldots \ldots$ Complementary polar angle of Compton cone axis, given by $\pi-$ $\alpha$ [radians]

c ..... Speed of light in vacuum $\left[\mathrm{m} \mathrm{s}^{-1}\right]$

$E_{\text {abs }} \ldots \ldots$ Energy deposition in absorber plane $[\mathrm{MeV}]$

$E_{\mathrm{b}} \ldots \ldots \ldots$ Binding energy of electron $[\mathrm{eV}]$

$E_{\gamma} \ldots \ldots$ Energy of photon $[\mathrm{MeV}]$

$E_{\gamma}^{\prime} \ldots \ldots$ Energy of scattered photon $[\mathrm{MeV}]$

$E_{\text {sca }} \ldots \ldots$ Energy deposition in scatter plane $[\mathrm{MeV}]$

$f_{\text {ang }}\left(\theta_{\text {axis }}\right) \ldots$ Unitless correction for response to a point source placed at angle $\theta_{\text {axis }}$ relative to central axis of detector.

$f_{\text {dist }}(r) \ldots . \quad$ Two-hit event-detection efficiency for a point source at a distance $r$ along the central axis of the detector [events decay ${ }^{-1}$ ]

$f_{i} \ldots \ldots$ Spatially-localized template of radioactivity $\left[\mathrm{Bq} \mathrm{m}^{-2}\right]$

$\tilde{f} \ldots \ldots$ Estimate of $f(x, y)$ produced by spatial-unfolding method [Bq $\left.\mathrm{m}^{-2}\right]$

$f(x, y) \ldots \ldots$ Radioactive source distribution $\left[\mathrm{Bq} \mathrm{m}^{-2}\right]$

$g_{i} \ldots \ldots \quad$ Spatial-response template to spatially-localized source $f_{i}$ [backprojections $\mathrm{s}^{-1}$ ]

€..... Unitless ratio of incident photon energy to the rest mass of the electron, $E_{\gamma} / m_{\mathrm{e}} c^{2}$

$\lambda \ldots \ldots$ Wavelength of photon $[\mathrm{m}]$

$M_{A} \ldots \ldots \quad$ Molar mass of material with atomic number $A\left[\mathrm{~kg} \mathrm{~mol}{ }^{-1}\right]$

$m_{\mathrm{e}} \ldots \ldots$ Mass of electron $[\mathrm{kg}]$ 


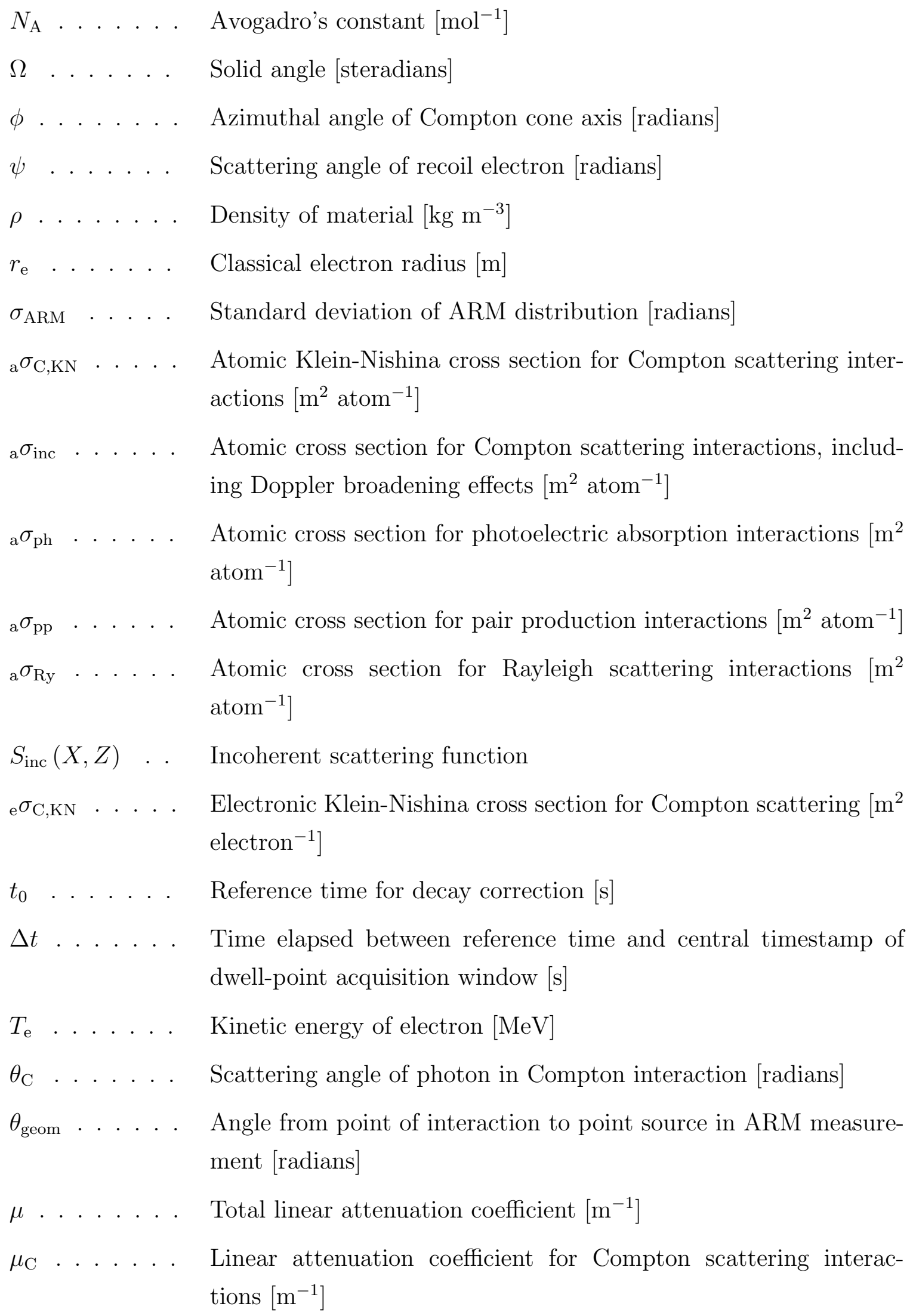


$\mu_{\mathrm{ph}} \ldots . . \quad$ Linear attenuation coefficient for photoelectric absorption interactions $\left[\mathrm{m}^{-1}\right]$

$\mu_{\mathrm{pp}}$.... Linear attenuation coefficient for pair production interactions $\left[\mathrm{m}^{-1}\right]$

$\mu_{\mathrm{Ry}}$.... Linear attenuation coefficient for Rayleigh scattering interactions $\left[\mathrm{m}^{-1}\right]$

$\mu_{\mathrm{tp}} \ldots . . . \quad$ Linear attenuation coefficient for triplet production interactions $\left[\mathrm{m}^{-1}\right]$

$Q$..... Energy released in a radioactive decay $[\mathrm{MeV}]$

$q_{\mathrm{e}} \ldots \ldots$ Charge of electron $[\mathrm{C}]$

$\vec{r}_{\mathrm{abs}} \ldots . . \quad$ Location of interaction in the absorber plane

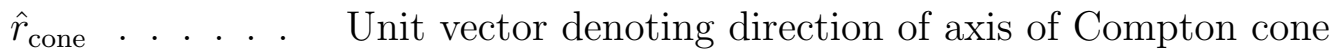

$\vec{r}_{\text {sca }} \ldots . . \quad$ Location of interaction in the scatter plane

$V_{\mathrm{bd}} \ldots \ldots$ Breakdown voltage in a SPAD $[\mathrm{V}]$

$V_{\text {bias }} \ldots . . \quad$ Operating bias of an SiPM $[\mathrm{V}]$

$V_{\text {over }} \ldots . . \quad$ Overvoltage applied to an SiPM [V]

$\hat{v}_{\mathrm{s}}$. . . . U Unit vector pointing from detector to point source in ARM measurement

$X \ldots$. . . Momentum-transfer parameter, $\sin \left(\theta_{\mathrm{C}} / 2\right) / \lambda\left[\mathrm{m}^{-1}\right]$

$Z$..... Atomic number [protons atom ${ }^{-1}$ ] 


\section{Contents}

Abstract $\quad$ ii

Acknowledgments $\quad$ iv

Statement of contribution vi

Published and presented works viii

List of abbreviations and symbols $\quad \mathrm{x}$

Contents $\quad$ xiv

List of Tables $\quad$ xix

List of Figures $\quad$ xx

1 Introduction 1

1.1 Motivation for research . . . . . . . . . . . . . . 1

1.2 Research objectives and hypotheses . . . . . . . . . . . . . 4

1.3 Organization of this thesis . . . . . . . . . . . 5

$\begin{array}{lll}2 & \text { Overview of required concepts } & 7\end{array}$

2.1 Radioactive decay . . . . . . . . . . . . . . . . . . 7

2.1.1 Alpha decay . . . . . . . . . . . . . . . . 7 
2.1.2 Beta decay and electron capture . . . . . . . . . . 8

2.1.3 Gamma decay and internal conversion . . . . . . . . . . 9

2.2 Interactions of gamma rays with matter . . . . . . . . . . . . 9

2.2.1 Photoelectric absorption . . . . . . . . . . . . . . 11

2.2.2 Rayleigh Scattering . . . . . . . . . . . . . . 12

2.2.3 Compton scattering . . . . . . . . . . . . . . . 13

Electron binding effects ............... 16

2.2.4 Pair production . . . . . . . . . . . . . . 16

Triplet production . . . . . . . . . . . . . . . 18

2.3 Compton gamma imaging . . . . . . . . . . . . . . . . 18

2.3.1 Angular resolution measure .............. 20

2.4 Scintillator physics . . . . . . . . . . . . . . . . 20

2.4.1 Energy spectrum in gamma-ray spectroscopy . . . . . . . . . . 21

2.4.2 Energy resolution .................. . . 23

2.4.3 Inorganic scintillators . . . . . . . . . . . . . . . . . . 24

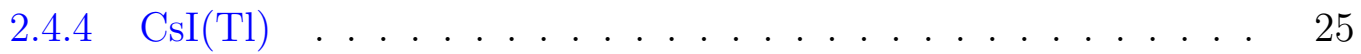

2.5 Silicon photomultipliers . . . . . . . . . . . . . . . . 27

2.5.1 p-type and n-type semiconductors . . . . . . . . . . . . 27

2.5.2 Semiconductor diodes . . . . . . . . . . . . . . . . . 29

2.5.3 Single-photon avalanche diodes . . . . . . . . . . . . 30

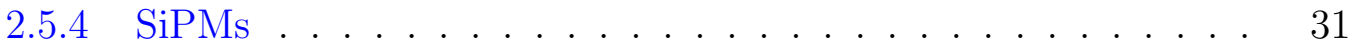

2.6 Natural background radioactivity . . . . . . . . . . . . . 35

2.7 Unfolding methods . . . . . . . . . . . . . . . . . . 43

$\begin{array}{lll}3 & \text { Instrumentation } & 46\end{array}$

3.1 The 3x3-module SCoTSS Compton gamma imager . . . . . . . . . 46 
3.2 Sensitive material: $\mathrm{CsI}(\mathrm{Tl}) \ldots \ldots \ldots$. . . . . . . . . . . 48

3.3 Light readout: SiPMs . . . . . . . . . . . . . . . . . 49

3.4 Detector electronics and data acquisition . . . . . . . . . . . 50

4 Common methodology $\quad 55$

4.1 Energy calibration ...................... 55

4.2 Event selection . . . . . . . . . . . . . . 56

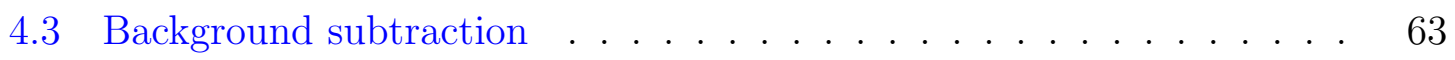

4.4 Making a Compton gamma image . . . . . . . . . . . . 63

4.5 Tomographic projection method . . . . . . . . . . 68

4.6 Monte Carlo representation of the 3x3-module SCoTSS Compton gamma imager . . . . . . . . . . . . . . . . 71

4.7 Point-model activity estimation . . . . . . . . . . . . 74

4.8 Spatial-unfolding method . . . . . . . . . . . 76

5 Point-source trial $\quad 83$

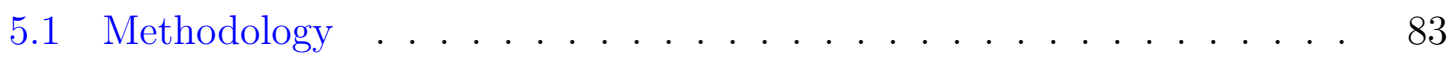

5.1.1 Experimental setup and data collection . . . . . . . . . 83

Data quality ................... 86

Event selection . . . . . . . . . . . . . . . 96

Individual dwell-point Compton gamma images . . . . . . . . 96

5.1.2 Estimating the position and orientation of the 3x3-module SCoTSS detector . . . . . . . . . . . . . . 102

5.1.3 Monte Carlo simulation of detector response . . . . . . . . . 103

Simulation geometry . . . . . . . . . . . . . 103

Comparison of MC simulation to real data . . . . . . . . 105

Point-model response . . . . . . . . . . . . . . . . 115 
Verification of tomographic reconstruction using MC simulation 117

Verification of point-model response method using MC simulation119

Response templates . . . . . . . . . . . . . . . . . 119

5.2 Results and discussion . . . . . . . . . . . . . . . . . . . . . 120

5.2.1 Uncorrected tomographic reconstruction . . . . . . . . . . 120

5.2.2 Point-model estimate . . . . . . . . . . . . . . . 123

5.2.3 Spatially-unfolded distribution . . . . . . . . . . . . . 123

Investigation of the effect of using a "tile source" in reconstructing a point source . . . . . . . . . . . . 127

Effects not included in simulated detector response . . . . . . 134

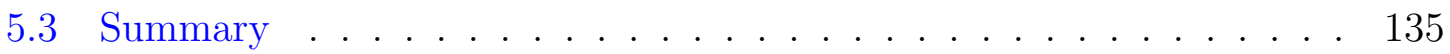

6 Spatially-extended source trial 136

6.1 Methodology ..................... 136

6.1.1 Experimental setup . . . . . . . . . . . . 136

6.1.2 Experimental data collection . . . . . . . . . . . . . . 139

Data quality . . . . . . . . . . . . . . . 141

Event selection . . . . . . . . . . . . . . . . . . 149

Individual dwell-point Compton gamma images . . . . . . . . 151

6.1.3 Estimating the position and orientation of the $3 \times 3$-module

SCoTSS detector . . . . . . . . . . . . . 156

6.1.4 Monte Carlo simulation of detector response . . . . . . . . . . 157

Simulation geometry . . . . . . . . . . . . 157

Comparison of MC simulation to real data . . . . . . . . . . 161

Point-model response . . . . . . . . . . . . . . . . . . . . 168

Verification of tomographic reconstruction using MC simulation 170 
Verification of point-model response method using MC simulation172

Response templates . . . . . . . . . . . . . . . . 172

6.2 Results and discussion . . . . . . . . . . . . . . . . . 173

6.2.1 Uncorrected tomographic reconstruction . . . . . . . . . . . 173

6.2 .2 Point-model estimate . . . . . . . . . . . . . . 176

6.2.3 Spatially-unfolded distribution . . . . . . . . . . . . 177

Tomographic fit . . . . . . . . . . . . . . . 177

Unfolded distribution and uncertainties . . . . . . . . . . . . . 182

Effect of environmental terrain not included in MC response . 190

6.3 Summary . . . . . . . . . . . . . . . . . . 195

$\begin{array}{llr}7 & \text { Conclusions and future work } & 197\end{array}$

7.1 Conclusion . . . . . . . . . . . . . . . . . . . 197

7.2 Future work . . . . . . . . . . . . . . . . . . 199

$\begin{array}{ll}\text { Appendices } & 201\end{array}$

A Relationship between attenuation coefficient and atomic cross sec$\begin{array}{ll}\text { tion } & 202\end{array}$

B Significance testing with the Z-score 204

$\begin{array}{ll}\text { References } & 206\end{array}$ 


\section{List of Tables}

5.1 Energy cuts used in event selection for the $662 \mathrm{keV}$ photons in the experimental point-source measurement. . . . . . . . . . 96

5.2 Number of events accepted at each dwell point in the experimental point-source measurement. . . . . . . . . . . . . . . . . . 996

5.3 Detector coordinates and orientations for the dwell-point positions during the experimental point-source measurement. . . . . . . . . . 103

5.4 Vertical back-projection angles used in the experimental point-source reconstruction. . . . . . . . . . . . . . . . . 120

6.1 Start times and measurement durations for experimental dwell-point measurements acquired during the Suffield trial. . . . . . . . . . . 140

6.2 Gamma-ray emissions of La-140. . . . . . . . . . . . . . 150

6.3 Energy cuts used in event selection for the $1.596 \mathrm{MeV}$ emission of La-140.151

6.4 Number of events accepted at each dwell point in the Suffield experiment.151

6.5 Detector coordinates and orientations for the experimental dwell-point positions during the Suffield trial. . . . . . . . . . . . 157

6.6 Total estimated activity and associated uncertainties from spatial unfolding of La-140 distribution in the Suffield experiment. . . . . . . 187

6.7 Composition of the soil assumed for attenuation calculations in the Suffield experiment. . . . . . . . . . . . . . . . . 190 


\section{List of Figures}

2.1 Mass attenuation coefficients for $\mathrm{CsI}(\mathrm{Tl}) \ldots \ldots \ldots \ldots$

2.2 Schematic illustration of photoelectric absorption. . . . . . . . 12

2.3 Schematic illustration of Compton scattering. . . . . . . . . . . 14

2.4 Polar plot of the differential electronic Klein-Nishina cross section. . . 15

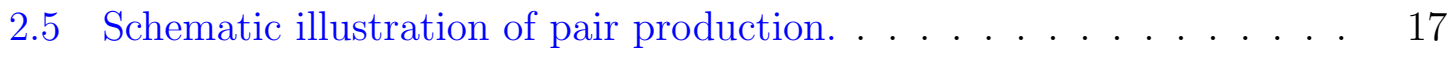

2.6 Illustration of Compton imaging on a representative two-plane detector design. . . . . . . . . . . . . . . . . . . . . . 19

2.7 Schematic of theoretical energy spectrum for a scintillator measuring monoenergetic gamma rays of energy $E_{\gamma} \ldots \ldots \ldots \ldots \ldots$

2.8 Illustration of the energy band structure for an inorganic scintillator. 25

2.9 Scintillation emission spectrum for $\mathrm{CsI}(\mathrm{Tl}) \ldots \ldots \ldots \ldots \ldots$

2.10 Temperature dependence of light output in $\mathrm{CsI}(\mathrm{Tl}) \ldots \ldots \ldots \ldots$

2.11 Illustration of a representative n-type material. . . . . . . . . 28

2.12 Illustration of a representative p-type material. . . . . . . . . . . 29

2.13 Illustration of semiconductor junctions for unbiased and reverse-biased diodes. . . . . . . . . . . . . . . . . . 30

2.14 Photon detection efficiency for an SiPM operated at an overvoltage of

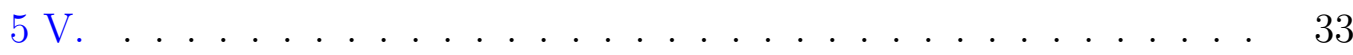

2.15 Dependence of SiPM dark-count rate on overvoltage. . . . . . . . . 34

2.16 Dependence of SiPM dark-count rate on temperature. . . . . . . . . 34 
2.17 U-238 decay series. . . . . . . . . . . . . . . . . . . . 36

2.18 Th-232 decay series. . . . . . . . . . . . . . . . . . 37

2.19 K-40 decay series. . . . . . . . . . . . . . . . . . . . . 38

2.20 Spectrum of $\mathrm{U}-238 \ldots \ldots \ldots \ldots \ldots$

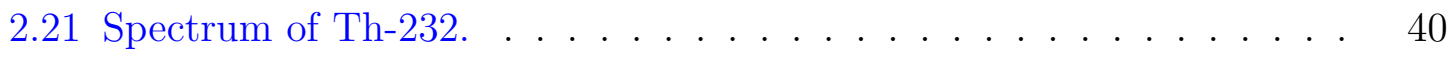

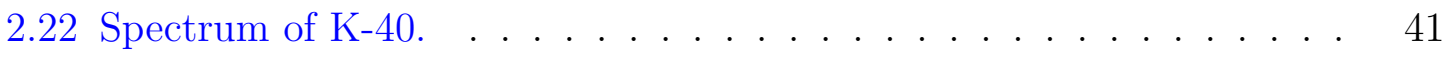

2.23 Spectrum of NORM background. . . . . . . . . . . . . . . 42

2.24 Relationship between measured energy spectra for synthetic Co-60 point source with and without detector response unfolded. . . . . . . 45

3.1 Interior view of the 3x3-module SCoTSS Compton gamma imager. . . 47

3.2 Examples of SiPM arrays used to read scintillator elements in the scatter plane of the 3x3-module SCoTSS detector. . . . . . . . . . . . 49

3.3 Pulse profile measured from a $\mathrm{CsI}(\mathrm{Tl}) / \mathrm{SiPM}$ unit irradiated by Cs-137. 51

3.4 Energy spectrum for two-hit events measured from a Cs-137 point source using the $3 \times 3$-module SCoTSS detector. . . . . . . . . . . 53

3.5 Representative Cs-137 energy spectra for single detector components for experimental data. . . . . . . . . . . . . . . . . . . 54

4.1 Example of an energy spectrum measured during calibration of the energy scale. . . . . . . . . . . . . . . . 56

4.2 Demonstration of gain drift correction for experimentally measured La-140 data. . . . . . . . . . . . . . . . . . . . . 60

4.3 Representative illustration of energy cuts used in event selection. . . . 61

4.4 Energy exchange in a Compton scattering event as a function of scattering angle. . . . . . . . . . . . . .

4.5 Coordinate system used for image reconstruction with the $3 \times 3$-module SCoTSS detector. . . . . . . . . . . . . . . . . . . . . . 64 
4.6 Coordinate system used for plotting Compton rings in the field of view of the detector. . . . . . . . . . . . . . . . . 65

4.7 Demonstration of the Compton gamma image reconstruction process for experimentally measured data . . . . . . . . . . . . . . . . . 67

4.8 Schematic of the components of a tomographic back-projection for a single ray. . . . . . . . . . . . . . . . . . .

4.9 Interior view of the geometry used in Monte Carlo simulations of the 3x3-module SCoTSS detector. . . . . . . . . . . . . . 72

4.10 Empirical energy resolution function used to smear energy deposits in Monte Carlo simulation. . . . . . . . . . . . . . . . . . . . 73

4.11 Schematic illustration of spatial-unfolding method. . . . . . . . . 78

4.12 Example of bootstrap resampling of Compton event data. . . . . . . . 82

5.1 Schematic representation of the experimental point-source measurement scenario. . . . . . . . . . . . . . . . . . . . . . . . . . . 84

5.2 LIDAR and 3x3-module SCoTSS detector used for the experimental point-source measurement. . . . . . . . . . . . . . . .

5.3 Energy spectrum at dwell point 0 in the experimental point-source measurement. . . . . . . . . . . . . . . . . . .

5.4 Energy spectrum at dwell point 1 in the experimental point-source measurement. . . . . . . . . . . . . . . . . 88

5.5 Energy spectrum at dwell point 2 in the experimental point-source measurement. . . . . . . . . . . . . . . . . .

5.6 Energy spectrum at dwell point 3 in the experimental point-source measurement. . . . . . . . . . . . . . . . . . 90

5.7 Energy spectrum at dwell point 4 in the experimental point-source measurement. . . . . . . . . . . . . . . . . . .

5.8 Energy spectrum at dwell point 5 in the experimental point-source measurement. . . . . . . . . . . . . . . . 
5.9 Energy spectrum at dwell point 6 in the experimental point-source measurement. . . . . . . . . . . . . . . . . . . . 93

5.10 Energy spectrum at dwell point 7 in the experimental point-source measurement. . . . . . . . . . . . . . . . .

5.11 Energy spectrum at dwell point 8 in the experimental point-source

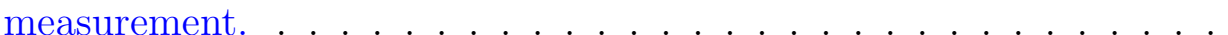

5.12 Field-of-view gamma image at dwell point 0 in the experimental pointsource measurement. . . . . . . . . . . . . . . . . .

5.13 Field-of-view gamma image at dwell point 1 in the experimental pointsource measurement. . . . . . . . . . . . . . . . . . . . . . . . . . . . 98

5.14 Field-of-view gamma image at dwell point 2 in the experimental pointsource measurement. . . . . . . . . . . . . . . . . . . . . 98

5.15 Field-of-view gamma image at dwell point 3 in the experimental pointsource measurement. . . . . . . . . . . . . . . . . . . . . .

5.16 Field-of-view gamma image at dwell point 4 in the experimental pointsource measurement. . . . . . . . . . . . . . . . . . .

5.17 Field-of-view gamma image at dwell point 5 in the experimental pointsource measurement. . . . . . . . . . . . . . . . . . . . . . . 100

5.18 Field-of-view gamma image at dwell point 6 in the experimental pointsource measurement.

5.19 Field-of-view gamma image at dwell point 7 in the experimental pointsource measurement. . . . . . . . . . . . . . . . . . . . . . . 101

5.20 Field-of-view gamma image at dwell point 8 in the experimental pointsource measurement. . . . . . . . . . . . . . . . . . . . . . 101

5.21 Point cloud of NRC laboratory generated by LIDAR scanner. . . . . . 102

5.22 View of the simulated 3x3-module SCoTSS detector geometry used for the experimental point-source measurement. . . . . . . . . . . . . . . 104

5.23 Comparison of simulated and experimental Compton imaging measurements for point source at dwell point $0 . \ldots$. . . . . . . . . . . 106 
5.24 Comparison of simulated and experimental Compton imaging measurements for point source at dwell point 1. . . . . . . . . . . . . . 107

5.25 Comparison of simulated and experimental Compton imaging measurements for point source at dwell point 2 . . . . . . . . . . . . . . . 108

5.26 Comparison of simulated and experimental Compton imaging measurements for point source at dwell point 3. . . . . . . . . . . . . . . 109

5.27 Comparison of simulated and experimental Compton imaging measurements for point source at dwell point 4. . . . . . . . . . . . . . . 110

5.28 Comparison of simulated and experimental Compton imaging measurements for point source at dwell point 5. . . . . . . . . . . . . . 111

5.29 Comparison of simulated and experimental Compton imaging measurements for point source at dwell point 6 . . . . . . . . . . . . . . . . 112

5.30 Comparison of simulated and experimental Compton imaging measurements for point source at dwell point 7. . . . . . . . . . . . . 113

5.31 Comparison of simulated and experimental Compton imaging measurements for point source at dwell point 8. . . . . . . . . . . . . . . . . 114

5.32 Point-response parametrization of a monoenergetic $662 \mathrm{keV}$ point source. 116

5.33 Tomographic reconstruction of synthetic monoenergetic $662 \mathrm{keV}$ point source with point-response approximation overlaid. . . . . . . . . . 118

5.34 ARM distribution for a synthetic monoenergetic $662 \mathrm{keV}$ point source. 121

5.35 Tomographic reconstruction of experimental point source with pointresponse approximation overlaid. . . . . . . . . . . . .

5.36 Tomographic reconstruction of experimentally-measured $662 \mathrm{keV}$ point source with fit produced by spatial unfolding. . . . . . . . . . . 125

5.37 Unfolded distribution with statistical error estimates for experimentally-measured $662 \mathrm{keV}$ point source. . . . . . . . . . . 126

5.38 Low-statistic tomographic reconstruction of synthetic $662 \mathrm{keV}$ point source with fit to data tomogram produced by spatial unfolding. . . . 129 
5.39 High-statistic tomographic reconstruction of synthetic $662 \mathrm{keV}$ point source with fit to data tomogram produced by spatial unfolding. . . . 130

5.40 Low-statistics tomographic reconstruction of synthetic $662 \mathrm{keV}$ "tile source" with fit to data tomogram produced by spatial unfolding. . . 131

5.41 High-statistics tomographic reconstruction of synthetic $662 \mathrm{keV}$ "tile source" with fit to data tomogram produced by spatial unfolding. . . 132

5.42 Comparison of spatially-unfolded synthetic point- and tile-source data. 133

6.1 Schematic representation of the Suffield experimental site. . . . . . . 137

6.2 Sprayer UGV used to deposit La-140 during the Suffield experiment. 139

6.3 Transportation of the 3x3-module SCoTSS detector using a BV206 tracked vehicle during the Suffield experiment. . . . . . . . . . . . . . 140

6.4 Energy spectrum at dwell point 0 in the Suffield experiment. . . . . . 143

6.5 Energy spectrum at dwell point 1 in the Suffield experiment. . . . . . 144

6.6 Energy spectrum at dwell point 2 in the Suffield experiment. . . . . . 145

6.7 Energy spectrum at dwell point 3 in the Suffield experiment. . . . . . 146

6.8 Energy spectrum at dwell point 4 in the Suffield experiment. . . . . . 147

6.9 Energy spectrum at dwell point 5 in the Suffield experiment. . . . . . 148

6.10 Field-of-view gamma image at dwell point 0 in the Suffield experiment. 152

6.11 Field-of-view gamma image at dwell point 1 in the Suffield experiment. 153

6.12 Field-of-view gamma image at dwell point 2 in the Suffield experiment. 153

6.13 Field-of-view gamma image at dwell point 3 in the Suffield experiment. 154

6.14 Field-of-view gamma image at dwell point 4 in the Suffield experiment. 154

6.15 Field-of-view gamma image at dwell point 5 in the Suffield experiment. 155

6.16 View of the simulated 3x3-module SCoTSS detector geometry used for the Suffield experiment. . . . . . . . . . . . . . . . . . . . . 158 
6.17 Experimentally-measured FWHM used to apply energy smearing in simulations of the Suffield experiment. . . . . . . . . . . . . 160

6.18 Comparison of simulated and experimental Compton imaging measurements for extended source at dwell point 0 . . . . . . . . . . . . . . 162

6.19 Comparison of simulated and experimental Compton imaging measurements for extended source at dwell point 1. . . . . . . . . . . . . 163

6.20 Comparison of simulated and experimental Compton imaging measurements for extended source at dwell point 2 . . . . . . . . . . . . . . . 164

6.21 Comparison of simulated and experimental Compton imaging measurements for extended source at dwell point 3. . . . . . . . . . . . . . . . 165

6.22 Comparison of simulated and experimental Compton imaging measurements for extended source at dwell point 4. . . . . . . . . . . . . 166

6.23 Comparison of simulated and experimental Compton imaging measurements for extended source at dwell point 5. . . . . . . . . . . . . 167

6.24 Point-response parametrization of an La-140 point source. . . . . . . 169

6.25 Tomographic reconstruction of synthetic La-140 "L-polygon" source with point-response approximation overlaid. . . . . . . . . . . . . 171

6.26 ARM distribution for a synthetic La-140 point source. . . . . . . . . . 174

6.27 Tomographic reconstruction of experimental La-140 source with pointresponse approximation overlaid. . . . . . . . . . . . . . . 175

6.28 Schematic representation of the Suffield experimental site in coordinates relative to south-western border. . . . . . . . . . . . . . 178

6.29 Tomographic reconstruction of experimentally-measured extended source with fit to data tomogram produced by spatial unfolding. . . . 180

6.30 Tomographic reconstruction and fit result for synthetic data made from simulation of unfolded La-140 distribution. . . . . . . . . . . . . . . . 181

6.31 Unfolded distribution of experimental La-140 distribution with systematic and statistical error maps. . . . . . . . . . . . . . . 183 
6.32 Components of systematic uncertainty on unfolded experimental La140 distribution. . . . . . . . . . . . . . . . . . . 186

6.33 Effect of snow and soil on measurable La-140 activity in the Suffield

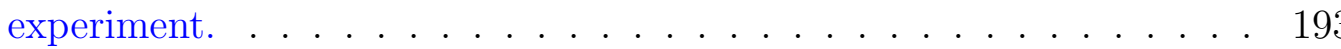

6.34 Unfolded distribution of synthetic La-140 L-shaped distribution with uniform surface activity concentration. . . . . . . . . . . . . . 194

B.1 Illustration of one-sided vs two-sided P-value. . . . . . . . . . . . 205 


\section{Chapter 1}

\section{Introduction}

\subsection{Motivation for research}

Since the discovery of radioactivity in the late $19^{\text {th }}$ century, advances in our understanding of atomic and nuclear energy have translated to useful everyday applications. These uses include nuclear power, radiology, nuclear medicine, product sterilization, food preservation, and more. However, the benefits of atomic and nuclear energy come with risks. For example, though occurrences of nuclear power plant disasters are low, the relative frequency of large-scale disasters remains large enough to be of continued concern. ${ }^{1}$ The Fukushima nuclear energy disaster of 2011 and the Chernobyl nuclear energy disaster of 1986 are perhaps the two most infamous cases. Lost radioactive sources, sometimes referred to as "orphan sources", may also present safety and security threats. If weaponized, for example by pairing the source with conventional explosives to form a "dirty bomb", the resulting fallout may cause negative health effects in victims and may contaminate the area in which the weapon was deployed. These examples showcase the value in developing instruments that can locate radioactive materials, as these instruments can be used in radiological safety and security applications to assist in the prevention of terrorist events or in the remediation of radiological accidents.

Most radioactive materials can be detected through the gamma rays that they emit, and this has resulted in a large body of research and a collection of technologies for the detection of radioisotopes from their gamma-ray emissions. Natural Resources Canada (NRCan) has developed technology for use on unmanned aerial 
vehicles, ${ }^{2}$ and has successfully used aerial-survey measurements with conventional aircraft in mapping radioactive contamination following detonation of a radiological dispersal device. ${ }^{3}$ Other groups have investigated a range of other detection methods for different scenarios. Mobile ground-based sensor networks have been investigated for the detection of anomalous radioactive materials. ${ }^{4-7}$ Networks of stationary detectors have been developed for localization and characterization of point sources. ${ }^{8}$ In any of these situations, pedestrial teams may be called in to perform finer searches of the area following the detection of radioactive materials. ${ }^{9}$ To remediate an area, for example in the late phase of a response to a large scale nuclear power disaster, further application of technology and analytical techniques may be required. ${ }^{10-14}$

To organize the radiological response capabilities of the Canadian government, the Federal Nuclear Emergency Plan (FNEP) ${ }^{15}$ was established. As part of the FNEP, NRCan is responsible for maintaining the capability to assess potential radiological hazards, which includes providing emergency response radiological surveying capabilities when required. Researchers from NRCan and the National Research Council Canada (NRC) partnered with researchers from McGill University prior to 2013 to develop Compton gamma imaging technologies capable of visualizing gamma-ray sources overlaid on an optical photograph of the environment. One of the detectors produced by this effort was based on bars of solid scintillator with conventional photomultipliers on either side for light readout and position reconstruction. ${ }^{16}$ The other detector was based on pixelated scintillation elements paired with silicon photomultipliers (SiPMs) for light readout. ${ }^{17,18}$ The SiPM-based detector developed by NRCan and the NRC was named the Silicon photomultiplier-based Compton Telescope for Safety and Security (SCoTSS), and became the technological foundation for the detector used in acquiring the measurements for this thesis. ${ }^{19}$ The SCoTSS detector technology employs pixelated $\mathrm{CsI}(\mathrm{Tl})$ scintillators coupled to SiPMs.

The SCoTSS detector is not the only Compton gamma imager designed for radiological safety and security operations. The Polaris-H Compton gamma imager series based on Cadmium Zinc Telluride (CZT) was developed by H3D (Ann Arbor, $\mathrm{MI})^{20}$ for use in radiological safety and security applications. The high efficiency multimode imager (HEMI) detector developed by researchers in Berkeley ${ }^{21}$ similarly employs CZT. CZT is a room-temperature compound semiconductor and can achieve better energy resolution than inorganic scintillators like $\operatorname{CsI}(\mathrm{Tl}) .{ }^{20,22,23}$ However, it 
is difficult to grow CZT crystals beyond a few $\mathrm{cm}$ in size, and doing so is more expensive than obtaining similarly-sized inorganic scintillators. High-purity Ge (HPGe) and $\mathrm{Si}(\mathrm{Li})$ semiconductors have also been investigated for use in Compton gamma imaging for safety and security purposes. ${ }^{24}$ While a detector based on HPGe and $\mathrm{Si}(\mathrm{Li})$ can achieve excellent energy resolution, its operation would rely on the use of cryogenic cooling systems, making such a device expensive and impractical for operation in varied operational conditions. Mechanical cooling options, such as Pelletier cooling ${ }^{25}$ may improve the ruggedness of a system that would otherwise need cryogens. However, mechanical cooling systems add cost and bulk to the detector. The choice to employ CsI $(\mathrm{Tl})$ with SiPMs in the SCoTSS detector allows for the production of a high-sensitivity and cost-effective gamma imager and spectrometer that is rugged enough to operate in real-world operational conditions.

Compton gamma imaging utilizes the physics of Compton scattering within the sensitive material of the detector to provide directional information regarding the origin of the gamma ray. The directional information provided by Compton gamma imagers is especially advantageous in situations where a detector cannot be brought into or over an area within which it is suspected that radioactive materials are present. One illustrative example of such a scenario would be an on-site inspection performed under the Comprehensive Nuclear-Test-Ban-Treaty (CTBT). Once the treaty enters into force, a member state suspected of having tested nuclear weapons technology would be required to submit to a search of the suspected test site by a team made up of personnel from other member states. The member state being investigated would be allowed to declare restricted-access sites, for example to protect sensitive information, provided that they are not individually larger than $4 \mathrm{~km}^{2}$ and do not exceed $50 \mathrm{~km}^{2}$ taken together. ${ }^{26}$ There are additional examples that could also arise outside of the context of the CTBT, such as in remediation efforts following nuclear energy disasters (e.g. in a situation similar to the Fukushima disaster) or terrorist attacks in which a "dirty bomb" containing radioactive materials is exploded. It may be difficult or dangerous to send manned or unmanned teams to survey within the site in the conditions following these kinds of incidents. In such scenarios, technologies and techniques that could reconstruct the spatial distribution and quantity of radioactivity inside of a restricted-access zone from measurements taken at the perimeter of the zone would be valuable. 
Compton gamma imaging is a modality well-suited to the reconstruction of a radioactive distribution from measurements taken at the perimeter of a restricted-access zone. The directional information from a Compton gamma image enables the use of tomographic reconstruction techniques. Tomographic reconstruction techniques are frequently used in fields such as medical imaging, and Compton gamma imaging has been investigated for use in tomographic applications in nuclear medicine. ${ }^{27}$ This thesis applies the fundamentals of tomographic imaging to radiological survey data from a Compton gamma imager, using the novel SCoTSS detector technology. Though the tomographic application of Compton gamma imaging presented in this thesis borrows from the fundamentals of tomographic back-projection methodology utilized in fields such as medical imaging, it is applied over much larger spatial extents and for data measured under outdoor operational conditions that are less controlled than the conditions found inside laboratories or hospitals. The development of tomographic reconstruction techniques that can be used to provide estimates of radioactive distributions in the field immediately post-acquisition, as well as advanced post-processing techniques that can be used to provide detailed quantitative localization of the distribution of radioactive materials from data acquired at the perimeter of a restrictedaccess zone, will assist NRCan in its efforts to provide radiological survey emergency response. We further anticipate that the methodology will find application in other end-use cases such as lost source, defence, medical imaging, nuclear safeguards, and nuclear weapons treaty verification.

\subsection{Research objectives and hypotheses}

A major research objective of this thesis is to demonstrate that the SCoTSS detector technology can be successfully deployed in experimental conditions mimicking the conditions which may be encountered in operational use for radiological response activities, such as in a CTBT on-site inspection. Additionally, this thesis focuses on the development of tomographic applications of Compton gamma imaging for use in radiological response. The techniques must be compatible with data acquired under operational conditions, which are frequently outdoors and less controlled than a laboratory setting. A result must be obtainable in the field immediately post-acquisition, presenting operators with a quick estimate of the distribution of radioactive materials 
and of the total activity contained within a restricted-access zone. A more detailed reconstruction made in post-processing should provide a map of the restricted-access zone showing spatially-localized quantitative activity estimates with systematic and statistical error estimates. To meet these outcomes, experimental studies of a simple point source and an extended source are required.

The specific hypotheses of this thesis are listed below:

1. The SCoTSS detector technology can be successfully deployed in realistic operational conditions.

2. Compton gamma imaging data acquired in realistic operational conditions, which may have low statistical power, can be used successfully to generate tomographic reconstructions that can guide operational efforts in near real time.

3. The response of the Compton gamma imager to a point source may be parameterized by simple functions generated using data simulated by Monte Carlo (MC), and this parametrization may be applied to experimental data to estimate the activity of an assumed point-source model. If the underlying distribution of radioactivity is spatially extended, the assumed point-source model activity estimate will agree with the activity of the source distribution.

4. A spatial-unfolding method can be successfully developed wherein a tomographic reconstruction of the data is fit with tomographic response templates produced by simulated data, allowing for determination of the quantity of radioactivity as a function of position.

\subsection{Organization of this thesis}

This thesis is organized into seven chapters. Chapter 2 provides an overview of the physics relevant to the research that was conducted. A description of gamma-ray interactions in materials is first presented, followed by the theory of Compton gamma imaging, the physics of inorganic scintillators and silicon photomultipliers, a review of naturally occurring radioactive materials, and methods of signal reconstruction by unfolding the response of a detector. Chapter 3 explains the detector technology and 
associated hardware that were used in data collection. Chapter 4 outlines methodology common to all chapters that follow it. It begins by describing the energy cuts required in data selection, and then describes the process of forming Compton images in the field of view of a Compton gamma imager. Forming tomographic images of the distribution of radioactive substances within a restricted-access zone by combining multiple sets of Compton imaging data is then described. The chapter closes with descriptions of how the detector was simulated with a MC toolkit and of the spatial-unfolding method developed to provide spatially-localized quantitative maps of the distribution of radioactivity within a restricted-access zone. Chapters 5 and 6 then present experimental trials of a point-source of Cs-137 and an extended-source of La-140 in a large-area restricted-access zone, respectively, including specific methodologies and discussions of the results. Chapter 7 concludes the thesis, providing closing statements and a summary of the results. 


\section{Chapter 2}

\section{Overview of required concepts}

The purpose of this chapter is to provide a review of the concepts related to the material in this thesis. The modes of radioactive decay are presented, followed by a discussion of the various ways in which gamma rays may interact with matter. The concept of Compton gamma imaging is then demonstrated. Following this, the physics of scintillators and silicon photomultipliers is discussed. Naturally occurring background radioactivity is then briefly reviewed. The chapter closes with a presentation of the principles of unfolding methods.

\subsection{Radioactive decay}

\subsubsection{Alpha decay}

Alpha decay occurs when a nucleus emits an alpha particle, which consists of two protons and two neutrons (i.e. a He-4 nucleus). This process occurs mainly in heavy nuclei. ${ }^{28}$ For an atomic species $\mathrm{X}$ with atomic number $Z, N$ neutrons, and mass number $A$, an alpha decay results in a new atomic species $\mathrm{Y}$ via

$$
{ }_{Z}^{A} \mathrm{X}_{N} \rightarrow{ }_{Z-2}^{A-4} \mathrm{Y}_{N-2}+\alpha .
$$

The energy released by a radioactive decay is denoted $Q$. The value of $Q$ for an alpha decay, $Q_{\alpha}$, goes into the kinetic energy of the alpha particle, $T_{\alpha}$, the kinetic energy of the recoiling daughter nucleus, $T_{\mathrm{Y}}$, and possibly to the energy of an excited state in which the daughter nucleus is formed, $E_{\mathrm{Y}}$ :

$$
Q_{\alpha}=T_{\alpha}+T_{\mathrm{Y}}+E_{\mathrm{Y}} .
$$




\subsubsection{Beta decay and electron capture}

There are two forms of beta decay. Beta-minus decay occurs when a nucleus emits a $\beta^{-}$particle, which is an electron. Beta-minus decay occurs within nuclei which tend to have an excess of neutrons, ${ }^{28}$ and follows a decay scheme of the form

$$
{ }_{Z}^{A} \mathrm{X}_{N} \rightarrow{ }_{Z+1}^{A} \mathrm{Y}_{N-1}+\beta^{-}+\bar{\nu}_{e}
$$

where $\bar{\nu}_{e}$ is an anti-electron neutrino. Beta-minus decay effectively converts a neutron into a proton. The energy released by a beta-minus decay, $Q_{\beta^{-}}$, is given by the sum of the maximum kinetic energy available to the electron emitted, $T_{\beta_{\max }^{-}}$, and the energy level of the daughter nucleus, $E_{\mathrm{Y}}$ :

$$
Q_{\beta^{-}}=T_{\beta_{\max }^{-}}+E_{\mathrm{Y}}
$$

The recoil energy of the daughter nucleus and the energy of the anti-electron neutrino is negligible and is omitted from equation $2.4 .^{28}$

Beta-plus decay occurs when a nucleus emits a $\beta^{+}$particle, which is a positron. Opposite to beta-minus decay, beta-plus decay involves the conversion of a proton into a neutron and usually occurs in nuclei with an excess of protons. ${ }^{28}$ This process follows the form

$$
{ }_{Z}^{A} \mathrm{X}_{N} \rightarrow{ }_{Z-1}^{A} \mathrm{Y}_{N+1}+\beta^{+}+\nu_{e}
$$

where $\nu_{e}$ is an electron neutrino. The value of $Q_{\beta^{+}}$is given by

$$
Q_{\beta^{+}}=T_{\beta_{\max }^{+}}+E_{\mathrm{Y}}+2 m_{\mathrm{e}} c^{2}
$$

where $m_{\mathrm{e}} c^{2}$ is the rest energy of an electron. Once again, the recoil energy of the daughter nucleus has been omitted in equation 2.6 because it is negligibly small. ${ }^{28}$

Electron capture decay is a process which competes with beta-plus decay, occurring within nuclei that have an abundance of protons. ${ }^{28}$ Electron capture involves the parent nucleus capturing one of the K- or L-shell electrons and converting a proton into a neutron and an electron neutrino:

$$
{ }_{Z}^{A} \mathrm{X}_{N} \rightarrow{ }_{Z-1}^{A} \mathrm{Y}_{N+1}+\nu_{e}
$$

The vacancy left by the inner-shell electron is replaced by an outer-shell electron. As the outer-shell electron drops into the inner-shell, it may either emit an x ray 
with an energy equal to the difference in energy levels of the two orbitals or it may transfer this energy to another electron. In the former case, the $\mathrm{x}$ rays emitted are referred to as characteristic $\mathrm{x}$ rays of that radioisotope because their energy signature is characteristic of that element. In the latter case, the process of one electron ejecting another electron by a transfer of energy in a transition is referred to as the Auger effect.

\subsubsection{Gamma decay and internal conversion}

In the alpha- and beta-decay processes, the daughter nucleus is frequently left in an excited state. In this situation, the excited daughter nucleus may decay to its ground state by emitting a gamma ray with an energy $E_{\gamma}$ equal to the difference in the energy levels of the excited state, $E_{\mathrm{Y}}$, and the ground state, $E_{\mathrm{Y}, \mathrm{g}}$ :

$$
E_{\gamma}=E_{\mathrm{Y}}-E_{\mathrm{Y}, \mathrm{g}}
$$

Transitions between intermediate energy levels are also possible, and in this case the value $E_{\mathrm{Y}, \mathrm{g}}$ in equation 2.8 is replaced by the energy of the intermediate energy level.

Internal conversion is a process that competes with gamma decay wherein an excited nucleus may transfer excess energy to one of its atomic electrons. The electron is then ejected from the atom with a kinetic energy $T_{\mathrm{e}}$ given by

$$
T_{\mathrm{e}}=\left(E_{\mathrm{Y}}-E_{\mathrm{Y}, \mathrm{g}}\right)-E_{\mathrm{b}}
$$

where $E_{\mathrm{b}}$ is the binding energy of the electron's orbital shell.

\subsection{Interactions of gamma rays with matter}

If a beam of $N_{0}$ gamma rays is incident upon a slab of material of finite thickness, the average number $N$ that will emerge on the other side of the slab without having undergone any interaction within the slab is given by

$$
N=N_{0} \exp (-\mu r)
$$

where $\mu$ is the linear attenuation coefficient of the material and $r$ is the path length of the beam in the material. The attenuation of the gamma rays is due to a combination of competing interaction processes, which occur with varying probabilities that 
are dependent on the energy of the gamma rays and on the material in which they interact. The total value of $\mu$ is made up of contributions from photoelectric absorption (denoted $\mu_{\mathrm{ph}}$ ), Rayleigh scattering (denoted $\mu_{\mathrm{Ry}}$ ), Compton scattering (denoted $\mu_{\mathrm{C}}$ ), and pair and triplet production (denoted $\mu_{\mathrm{pp}}$ and $\mu_{\mathrm{tp}}$, respectively). The linear attenuation coefficients are related by

$$
\mu=\mu_{\mathrm{ph}}+\mu_{\mathrm{Ry}}+\mu_{\mathrm{C}}+\mu_{\mathrm{pp}}+\mu_{\mathrm{tp}} .
$$

As will be discussed in further detail in chapter 3, the detector used to acquire the data presented in this thesis used $\mathrm{CsI}(\mathrm{Tl})$ scintillators as the sensitive material. Figure 2.1 shows the density-normalized values of the linear attenuation coefficient, known as the mass attenuation coefficient, for each of these interaction processes as well as the total mass attenuation coefficient in $\mathrm{CsI}(\mathrm{Tl})$ for gamma rays of energies ranging from $0.001 \mathrm{MeV}$ to $1000 \mathrm{MeV}$, presented on a logarithmic scale. A description of each of the interaction processes is given in the following subsections.

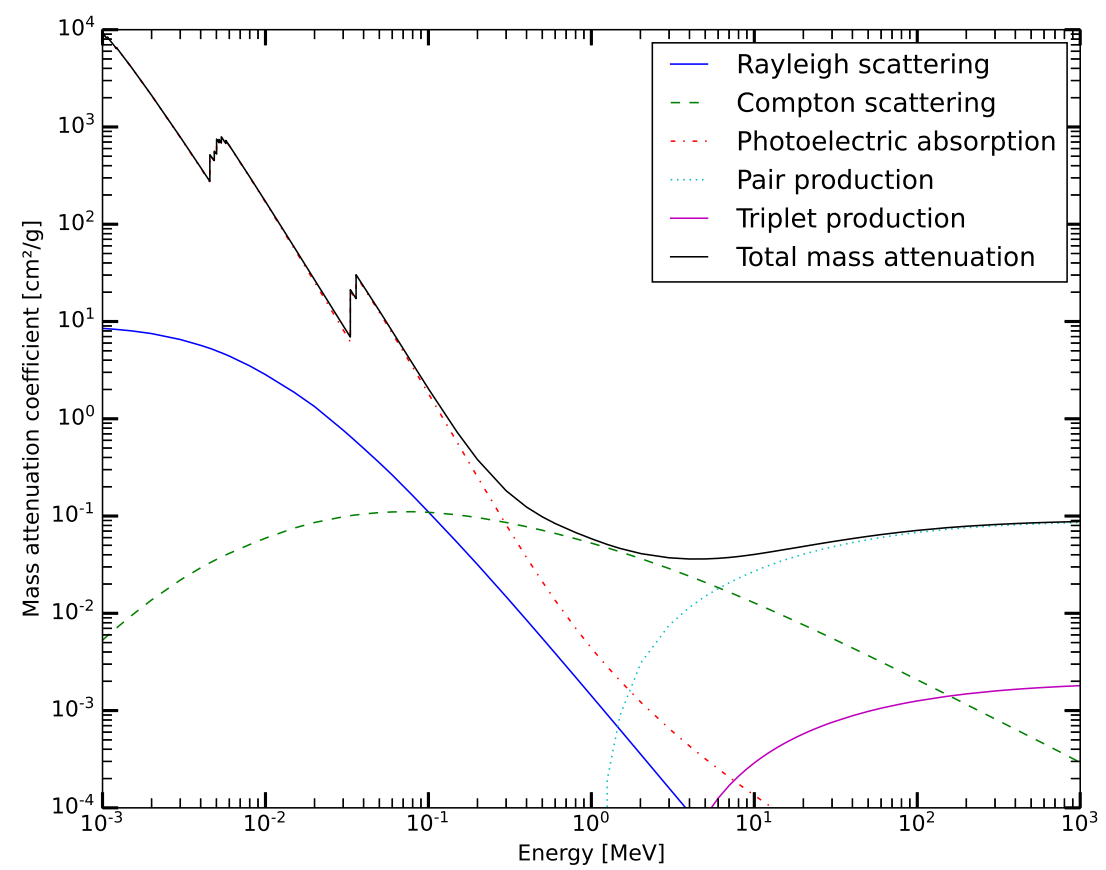

Figure 2.1: Mass attenuation coefficients for interaction processes in CsI(Tl) as a function of photon energy. This figure was generated using data from the XCOM database. ${ }^{29}$

Equation 2.10 can be derived from fundamental interaction probabilities de- 
scribed by the atomic cross sections ${ }_{\mathrm{a}} \sigma$ for each interaction process as a particle traverses a slab of infinitesimal thickness; the full derivation is given in appendix A. The linear attenuation coefficient $\mu$ is related to ${ }_{\mathrm{a}} \sigma$ through the number density of atoms in a sample:

$$
\mu=\left({ }_{\mathrm{a}} \sigma\right) \frac{\rho N_{\mathrm{A}}}{M_{A}}
$$

where $M_{A}$ is the molar mass of the material, $\rho$ is the density of the material, and $N_{\mathrm{A}}$ is Avogadro's constant and is equal to $6.022 \times 10^{23} \mathrm{~mol}^{-1}$.

For processes where the interactions take place with electrons, descriptions in terms of the electronic cross section ${ }_{\mathrm{e}} \sigma$ are frequently used. A neutral atom with atomic number $Z$ has $Z$ electrons, and the relationship between ${ }_{\mathrm{a}} \sigma$ and $\mathrm{e}_{\mathrm{e}} \sigma$ is simply

$$
{ }_{\mathrm{a}} \sigma=\left({ }_{\mathrm{e}} \sigma\right) Z
$$

\subsubsection{Photoelectric absorption}

A photoelectric absorption interaction involves an interaction between an incident gamma ray and an atomic electron. ${ }^{28}$ Figure 2.2 shows a schematic illustration of the photoelectric effect. Electrons, represented as grey circles, orbit the atomic nucleus in their atomic shells, represented as a blue circle and concentric black rings respectively. An incident photon, illustrated with a wavy red line, carries energy $E_{\gamma}$ and interacts with an electron that is bound to the atom with a binding energy $E_{\mathrm{b}}$. If $E_{\gamma}>E_{\mathrm{b}}$, the photon can give all of its energy to the electron which is then ejected from the atom, indicated in figure 2.2 as a solid purple arrow. Assuming that the kinetic energy imparted to the atom is negligible, the electron is ejected with kinetic energy $T_{\mathrm{e}}$ given by

$$
T_{\mathrm{e}}=E_{\gamma}-E_{\mathrm{b}}
$$

Notable in figure 2.1 is the presence of discontinuities in the mass attenuation coefficient for the photoelectric absorption interaction process. These discontinuities occur whenever the energy of the incident photon is equal to the binding energy of an atomic orbital level of one of the atomic species in the sample, namely Cs or I in the case of $\mathrm{CsI}(\mathrm{Tl})$. Depending on whether these discontinuities are associated with the binding energy of the K-shell, L-shell, or M-shell of the atomic species, the 


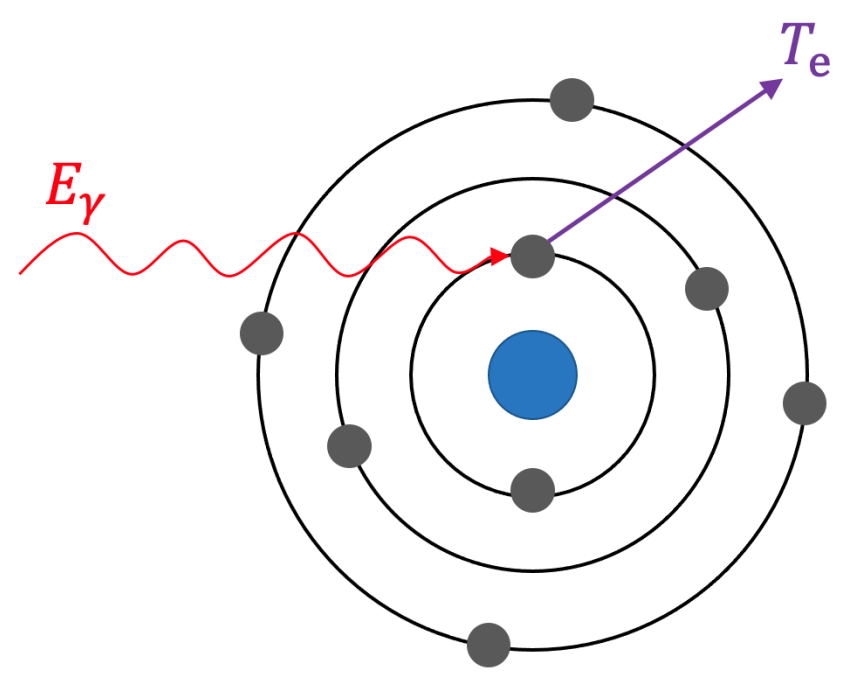

Figure 2.2: Schematic illustration of photoelectric absorption. Atomic electrons are illustrated as grey circles, atomic shells as black rings, the atomic nucleus as a blue circle, the incident photon by the solid red wavy line, and the trajectory of the recoil electron by the purple arrow.

discontinuities are referred to as the K-edge, L-edge, or M-edge; these may be further broken down into contributions from subshells. K-edge and L-edge effects are visible in figure 2.1 at energies of approximately $0.035 \mathrm{MeV}$ and $0.0055 \mathrm{MeV}$, respectively. ${ }^{29}$

Figure 2.1 shows that photoelectric absorption is the dominant interaction process in $\mathrm{CsI}(\mathrm{Tl})$ for low energies, up to approximately $0.3 \mathrm{MeV}$. The atomic cross section for photoelectric absorption, ${ }_{\mathrm{a}} \sigma_{\mathrm{ph}}$, is strongly dependent on the atomic number $Z$ and on the reciprocal of the energy of the incident photon. It may be approximately described as

$$
{ }_{\mathrm{a}} \sigma_{\mathrm{ph}} \stackrel{\propto}{\sim} \frac{Z^{n}}{E_{\gamma}^{m}}
$$

where $n$ is a constant varying between 4 and 5 as $E_{\gamma}$ increases, and $m$ is a constant varying between 3 and 1 as $E_{\gamma}$ increases. ${ }^{30}$ In general, photoelectric absorption dominates at low energies.

\subsubsection{Rayleigh Scattering}

Rayleigh scattering, also known as coherent scattering, occurs when an incident photon is scattered by interacting with the entire ensemble of electrons orbiting an atom. ${ }^{28}$ A Rayleigh scattering interaction does not result in the ejection of an electron from 
the atom, nor does it involve an electron being raised to an excited state. Because the photon interacts with the entire orbital cloud of electrons, the entire atom absorbs the momentum that is transferred from the photon; due to the relatively large mass of an atom, the energy transferred is negligible and the scattered photon has essentially the same energy as the incident photon.

Figure 2.1 shows that Rayleigh scattering is more likely than Compton scattering for energies below approximately $0.1 \mathrm{MeV}$ in $\mathrm{CsI}(\mathrm{Tl})$, but remains less probable than a photoelectric interaction. The atomic cross section, a $\sigma_{\mathrm{Ry}}$, for Rayleigh scattering behaves as ${ }^{30}$

$$
{ }_{\mathrm{a}} \sigma_{\mathrm{Ry}} \propto \frac{Z^{2}}{E_{\gamma}^{2}} .
$$

In general, Rayleigh scattering is a significant interaction channel at low values of $E_{\gamma}$ and at high $Z$.

\subsubsection{Compton scattering}

Compton scattering occurs when a photon interacts with an electron and transfers an amount of energy that exceeds the binding energy of the electron, producing a recoil electron and a scattered photon. ${ }^{28}$ Figure 2.3 provides a schematic illustration of a Compton scattering interaction in which electrons, represented as grey circles, orbit the atomic nucleus in their atomic shells, illustrated as a blue circle and concentric black rings respectively. An incoming photon, represented as a solid wavy red line, carries energy $E_{\gamma}$ and interacts with an electron. The photon imparts some of its energy to the electron, which is ejected from the atom at an angle $\psi$ relative to the trajectory of the incident photon, indicated by the solid purple line. The photon is scattered through an angle $\theta_{\mathrm{C}}$ and departs with energy $E_{\gamma}^{\prime}<E_{\gamma}$, represented by the dashed wavy red line.

The kinematics of Compton scattering can be employed to reconstruct the direction of an incoming gamma ray; this will be discussed in section 2.3. Because the binding energy of the electron that takes part in a Compton scattering interaction is typically small, an approximation is made in the equations presented in this section that the electron is unbound. Additionally, it is assumed that the electron momentum may be neglected. These assumptions become more accurate when $E_{\gamma} \gg E_{\mathrm{b}}$. Under these assumptions, conservation of energy and of momentum results in the following 


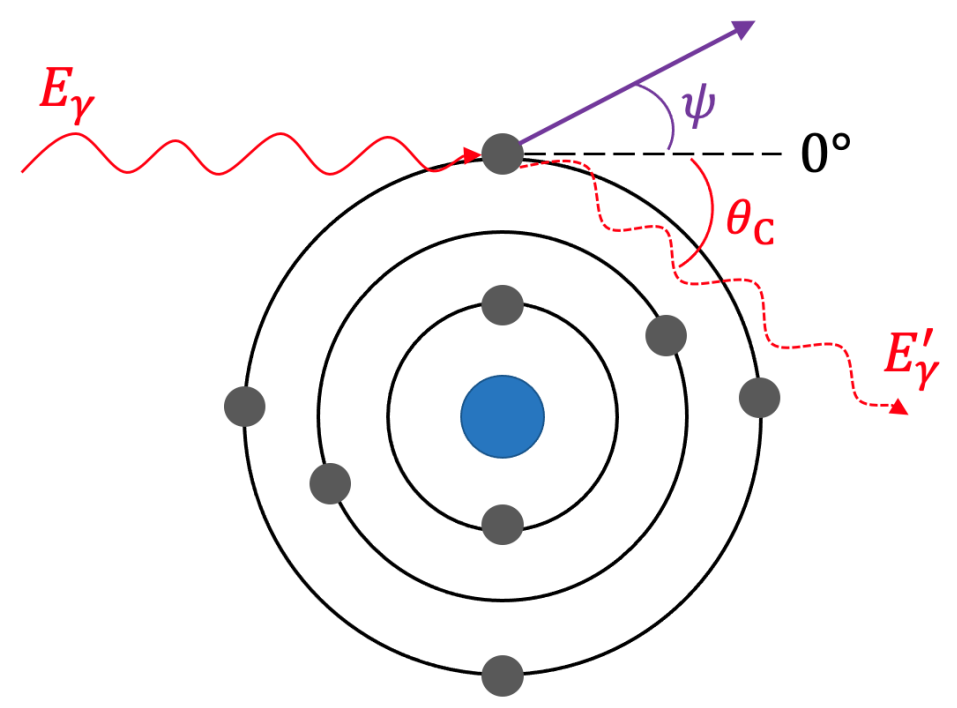

Figure 2.3: Schematic illustration of Compton scattering. Atomic electrons are illustrated as grey circles, atomic shells as black rings, the atomic nucleus as a blue circle, the incident photon by the solid red wavy line, the scattered photon by the dashed red wavy line, and the trajectory of the recoil electron by the purple arrow.

relationship between the incoming and outgoing photon energies:

$$
E_{\gamma}^{\prime}=\frac{E_{\gamma}}{1+\epsilon\left(1-\cos \theta_{\mathrm{C}}\right)}
$$

where $\epsilon=E_{\gamma} / m_{\mathrm{e}} c^{2}$, and $m_{\mathrm{e}} c^{2}$ is the rest energy of the electron. ${ }^{28}$ The kinetic energy transferred to the recoil electron is given by

$$
T_{\mathrm{e}}=E_{\gamma}-E_{\gamma}^{\prime}
$$

Figure 2.1 shows that Compton scattering is the dominant interaction process in $\mathrm{CsI}(\mathrm{Tl})$ for energies between approximately $0.3 \mathrm{MeV}$ and $6 \mathrm{MeV}$. In general, Compton scattering interactions dominate at intermediate energies. The differential electronic cross section for Compton scattering for unbound electrons at rest can be described by the Klein-Nishina formula. ${ }^{28}$ The differential electronic Klein-Nishina cross section ${ }_{\mathrm{e}} \sigma_{\mathrm{C}, \mathrm{KN}}$ per unit solid angle $\Omega$ is given by

$$
\frac{\mathrm{d}_{\mathrm{e}} \sigma_{\mathrm{C}, \mathrm{KN}}}{\mathrm{d} \Omega}=\frac{r_{\mathrm{e}}^{2}}{2}\left(1+\cos ^{2} \theta_{\mathrm{C}}\right) \frac{1}{\left[1+\epsilon\left(1-\cos \theta_{\mathrm{C}}\right)\right]^{2}}\left[1+\frac{\epsilon^{2}\left(1-\cos \theta_{\mathrm{C}}\right)^{2}}{\left[1+\epsilon\left(1-\cos \theta_{\mathrm{C}}\right)\right]\left(1+\cos ^{2} \theta_{\mathrm{C}}\right)}\right]
$$

where $r_{\mathrm{e}}$ is the classical electron radius and is approximately equal to $2.82 \mathrm{fm}$. Substitution of $\mathrm{d} \Omega=2 \pi \sin \theta_{\mathrm{C}} \mathrm{d} \theta_{\mathrm{C}}$ into equation 2.19 gives the electronic Klein-Nishina 
cross section per unit photon scattering angle:

$$
\frac{\mathrm{d}_{\mathrm{e}} \sigma_{\mathrm{C}, \mathrm{KN}}}{\mathrm{d} \theta_{\mathrm{C}}}=2 \pi \sin \theta_{\mathrm{C}} \frac{\mathrm{d}_{\mathrm{e}} \sigma_{\mathrm{C}, \mathrm{KN}}}{\mathrm{d} \Omega} .
$$

Figure 2.4 shows polar plots of equation 2.19 for several values of $E_{\gamma}$ in units of $\mathrm{mb} / \mathrm{sr} /$ electron along the increasing radial, where the polar angle represents $\theta_{\mathrm{C}}$. As the value of $E_{\gamma}$ increases, photons undergoing Compton scattering interactions scatter at shallower angles.

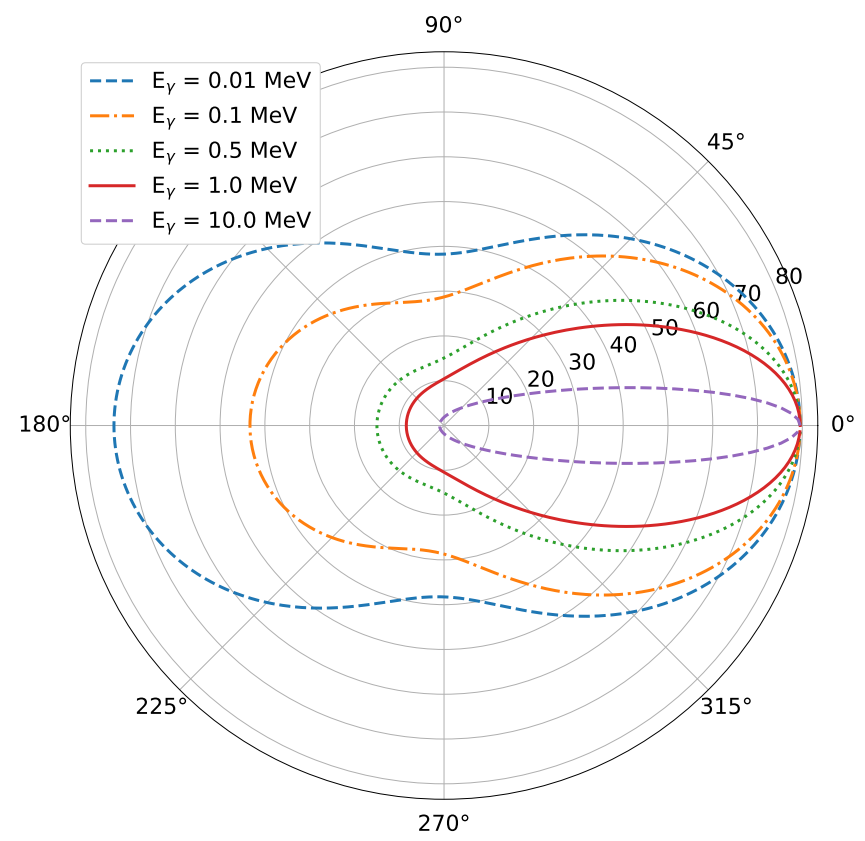

Figure 2.4: Polar plot of the differential electronic Klein-Nishina cross section per unit solid angle, $\frac{\mathrm{d}_{\mathrm{e}} \sigma_{\mathrm{C}, \mathrm{KN}}}{\mathrm{d} \Omega}$, for various values of $E_{\gamma}$. The units along the radial component of the plot are mb/sr/electron.

The electronic Klein-Nishina cross section is obtained by integrating equation 2.19 over the full space of solid angles into which a photon may scatter, yielding:

$$
{ }_{\mathrm{e}} \sigma_{\mathrm{C}, \mathrm{KN}}=2 \pi r_{\mathrm{e}}^{2}\left[\frac{1+\epsilon}{\epsilon^{2}}\left(\frac{2(1+\epsilon)}{1+2 \epsilon}-\frac{\ln (1+2 \epsilon)}{\epsilon}\right)+\frac{\ln (1+2 \epsilon)}{2 \epsilon}-\frac{1+3 \epsilon}{(1+2 \epsilon)^{2}}\right] .
$$

For an atom with $Z$ electrons, the atomic Klein-Nishina cross section ${ }_{\mathrm{a}} \sigma_{\mathrm{C}, \mathrm{KN}}$ is:

$$
{ }_{\mathrm{a}} \sigma_{\mathrm{C}, \mathrm{KN}}=\left({ }_{\mathrm{e}} \sigma_{\mathrm{C}, \mathrm{KN}}\right) Z
$$


The behaviour of ${ }_{\mathrm{a}} \sigma_{\mathrm{C}, \mathrm{KN}}$ is of particular important as it influences the design of a Compton gamma imager.

\section{Electron binding effects}

Derivation of the Klein-Nishina cross sections assumes stationary and unbound electrons. Under conditions when $E_{\gamma} \approx E_{\mathrm{b}}$, these assumptions begin to break down and the effect of the non-zero binding energy and momentum of the electron is noticeable. This results in the Doppler broadening effect, wherein the distributions of $\theta_{\mathrm{C}}$, $\psi$, and $T_{\mathrm{e}}$ are broadened around their expected values. The broadening of the distribution of $\theta_{\mathrm{C}}$ results in a smearing of image reconstructions for Compton gamma imagers which cannot measure the kinematic properties of the scattered electron in a Compton interaction.

The differential electronic Klein-Nishina coefficient given by equation 2.19 can be amended to include electron binding and momentum effects with the use of the incoherent scattering function $S_{\text {inc }}(X, Z)$ as

$$
\frac{\mathrm{d}_{\mathrm{a}} \sigma_{\mathrm{inc}}}{\mathrm{d} \Omega}=\frac{\mathrm{d}_{\mathrm{e}} \sigma_{\mathrm{C}, \mathrm{KN}}}{\mathrm{d} \Omega} S_{\mathrm{inc}}(X, Z)
$$

where ${ }_{\mathrm{a}} \sigma_{\mathrm{inc}}$ is the atomic incoherent-scattering cross section, and $X=\sin \left(\theta_{\mathrm{C}} / 2\right) / \lambda$ is the momentum-transfer parameter for a photon of wavelength $\lambda$. Further details of $S_{\text {inc }}(X, Z)$ are outside the scope of this thesis, and the reader is directed to reference 28 for more information.

\subsubsection{Pair production}

Pair production occurs when a photon interacts with the Coulomb field of an atomic nucleus, disappearing and giving rise to an electron and a positron. ${ }^{28}$ Figure 2.5 shows a schematic illustration of a pair production interaction. An incident photon of energy $E_{\gamma}$, represented by the wavy red line, approaches an atomic nucleus, represented by the blue circle. The photon interacts with the Coulomb field of the nucleus, illustrated by the orange wavy line, and produces an electron and a positron, represented by a grey and green circle, respectively. The electron recoils at an angle $\psi_{\mathrm{e}^{-}}$with kinetic energy $T_{\mathrm{e}^{-}}$, indicated by the purple arrow, while the positron recoils at an angle $\psi_{\mathrm{e}^{+}}$ with kinetic energy $T_{\mathrm{e}^{+}}$, indicated by the black arrow. 


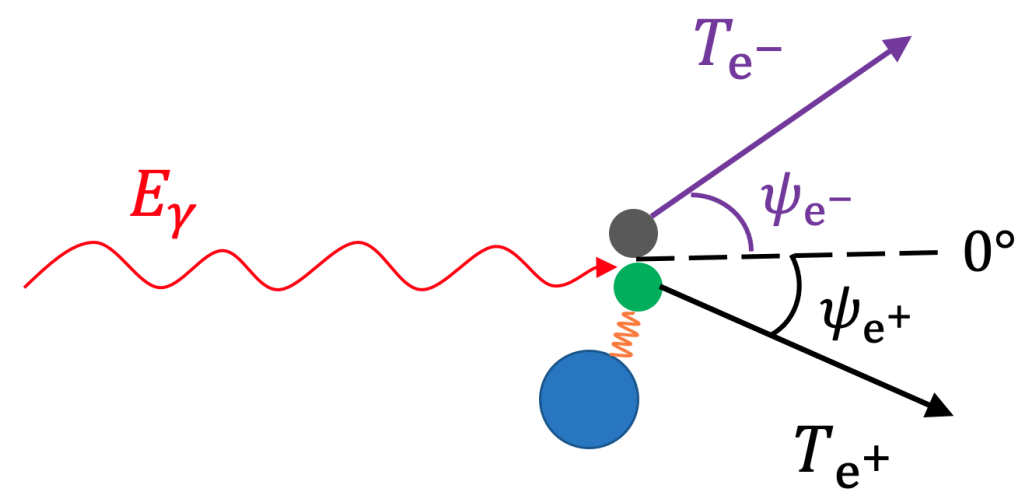

Figure 2.5: Schematic illustration of pair production. The atomic nucleus is represented by the blue circle, the incident photon is represented by the solid red wavy line, the interaction of the photon with the nuclear Coulomb force field is represented by the orange wavy line, the electron produced is represented by the grey circle with trajectory represented by the purple arrow, and the positron produced is represented by the green circle with trajectory represented by the black arrow.

Pair production has a threshold energy of $2 m_{\mathrm{e}} c^{2}$. When a pair production interaction occurs, the kinetic energies imparted to the electron and the positron are in general not equal. ${ }^{28}$ The average energy $\bar{T}$ received by each of the electron and the positron is

$$
\bar{T}=\frac{E_{\gamma}-2 m_{\mathrm{e}} c^{2}}{2} .
$$

The average recoil angle $\bar{\psi}$ is dependent on the reciprocal of $\bar{T}$ :

$$
\bar{\psi}=\frac{m_{\mathrm{e}} c^{2}}{\bar{T}}
$$

The atomic cross section of pair production interactions, ${ }_{\mathrm{a}} \sigma_{\mathrm{pp}}$, is given by

$$
{ }_{\mathrm{a}} \sigma_{\mathrm{pp}}=\alpha r_{\mathrm{e}}^{2} Z^{2} F_{\mathrm{pp}}\left(E_{\gamma}, Z\right)
$$

where $\alpha$ is the fine structure constant and $F_{\mathrm{pp}}\left(E_{\gamma}, Z\right)$ is a function dependent on $E_{\gamma}$ and $Z ;^{30}$ the details of $F_{\mathrm{pp}}\left(E_{\gamma}, Z\right)$ are beyond the scope of this thesis. In general, pair production becomes the dominant interaction mode at higher values of $E_{\gamma}$. Figure 2.1 shows that pair production dominates at high values of $E_{\gamma}$, exceeding Compton scattering interactions in $\mathrm{CsI}(\mathrm{Tl})$ past energies of approximately $6 \mathrm{MeV}$. Pair production is not a dominant interaction channel for the range of radioisotope energies involved in this thesis. 


\section{Triplet production}

A phenomenon similar to pair production may occur when a photon interacts in the Coulomb field of an electron. This is known as triplet production, and it requires a minimum energy of $4 m_{\mathrm{e}} c^{2} .30$

\subsection{Compton gamma imaging}

Compton gamma imaging is an approach that uses the kinematics of Compton scattering to infer directional information from an incident gamma ray. Figure 2.6 demonstrates the principles of Compton gamma imaging for a representative two-plane detector design, similar to the detector design that was used for the work presented in this thesis and that will be discussed in chapter 3. The detector contains two position- and energy-sensitive planes, represented in figure 2.6 by two yellow rectangular prisms. A gamma-ray source, represented by a magenta star, emits a photon of energy $E_{\gamma}$, represented by a magenta arrow, towards the detector. The photon interacts at position $\vec{r}_{\text {sca }}$ in the first detector plane and ideally undergoes a single Compton scattering interaction, depositing energy $E_{\text {sca }}$ in the detector. The photon scatters through an angle $\theta_{\mathrm{C}}$ with energy $E_{\gamma}^{\prime}=E_{\gamma}-E_{\text {sca }}$. Ideally, the photon then undergoes a photoelectric absorption interaction in the second detector plane, depositing its remaining energy $E_{\text {abs }}=E_{\gamma}^{\prime}$ at location $\vec{r}_{\text {abs }}{ }^{*}$. Because of the desired interaction process in each detector plane, the first plane is referred to as the scatter plane and the second plane is referred to as the absorber plane. The measured interactions define a conical surface of half-opening angle $\theta_{\mathrm{C}}$ about an axis aligned with the unit vector $\hat{r}_{\text {cone }}$, where $\hat{r}_{\text {cone }}$ is given by

$$
\hat{r}_{\text {cone }}=\frac{\vec{r}_{\mathrm{sca}}-\vec{r}_{\mathrm{abs}}}{\left\|\vec{r}_{\mathrm{sca}}-\vec{r}_{\mathrm{abs}}\right\|} .
$$

The path of the incident gamma ray must lie along this conical surface, which has been illustrated as a blue cone in figure 2.6. Using equation 2.17, the Compton scattering angle may be calculated from the measured energy depositions as:

$$
\cos \theta_{\mathrm{C}}=1+m_{\mathrm{e}} c^{2}\left(\frac{1}{E_{\mathrm{sca}}+E_{\mathrm{abs}}}-\frac{1}{E_{\mathrm{abs}}}\right) .
$$

*It can also happen that the photon undergoes another scattering event and escapes the detector without having deposited all of its energy, though the detector is designed to minimize the probability of this occurring. 
Each gamma ray that undergoes a "two-hit" event such as that described above defines another conical surface. Two additional representative cones are indicated in figure 2.6 by red and green surfaces. In the absence of Doppler broadening effects discussed in section 2.2.3, and if the detector has perfect position and energy resolution, the cones will intersect at the location of the gamma-ray source, allowing its position to be determined.

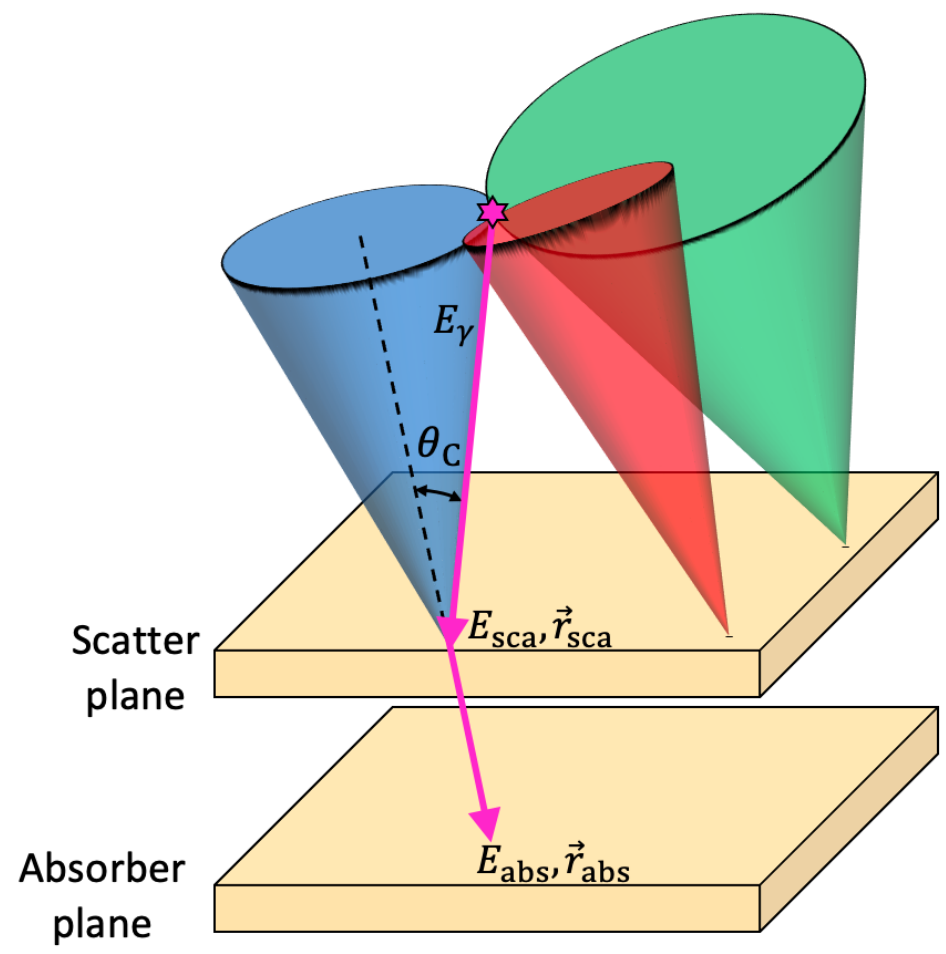

Figure 2.6: Illustration of Compton imaging on a representative two-plane detector design. The yellow rectangular prisms represent the two detector planes. A gammaray source, represented by the magenta star, emits a gamma ray of energy $E_{\gamma}$ that scatters within the first detector plane and is absorbed in the second detector plane, represented by the solid magenta arrows. The interaction locations $\vec{r}_{i}$ and energy deposits $E_{i}$ define the axis and half-opening angle $\theta_{\mathrm{C}}$, respectively, of a conical surface illustrated in blue. Two additional representative conical surfaces that arise from additional gamma rays are illustrated in green and red.

When designing a Compton gamma imager, the Compton scattering and photoelectric absorption interactions discussed in section 2.2 are of prime importance. In the discussion of Compton gamma imaging presented above for a representative two-plane detector, it was assumed that the measurement of $E_{\text {sca }}$ resulted from a 
single Compton scattering interaction in the scatter plane, whereas the measurement of $E_{\text {abs }}$ resulted from a complete capture of the scattered photon via a photoelectric interaction. For such a detector design, the materials and geometry must therefore be chosen in order to maximize the likelihood of an incident photon to undergo a single Compton scattering interaction in the scatter plane and then leave without further interaction. Similarly, the absorber plane must be designed in order to maximize the area into which a scattered photon may cross into, and to provide a high likelihood that the photon will deposit all of its energy locally via photoelectric absorption. These effects are energy-dependent, and optimization of the parameters of the detector may be achieved by MC simulation studies. ${ }^{31}$

\subsubsection{Angular resolution measure}

The angular resolution of a Compton gamma imager is quantified by the angular resolution measure (ARM) using measurements of a point source. Let $\hat{v}_{\mathrm{s}}$ be the unit vector which points from the interaction site $\vec{r}_{\text {sca }}$ in the scatter plane to the point source. The angle $\theta_{\text {geom }}$ from the point of interaction in the detector to the source is given by

$$
\theta_{\text {geom }}=\arccos \left(\hat{v}_{\mathrm{s}} \cdot \hat{r}_{\text {cone }}\right)
$$

where $\hat{r}_{\text {cone }}$ is given by equation 2.27. The ARM is calculated as

$$
A R M=\theta_{\mathrm{C}}-\theta_{\text {geom }}
$$

Because $\theta_{\mathrm{C}}$ is reconstructed from the measured energy deposits and $\theta_{\text {geom }}$ is reconstructed from the interaction locations, the ARM combines both the energy and spatial resolution of the detector into a single metric. Each accepted Compton event contributes a single ARM measurement. The standard deviation $\sigma_{\mathrm{ARM}}$ of the distribution of the ARM values may be used to characterize the precision of the detector.

\subsection{Scintillator physics}

A scintillator is a material capable of converting the kinetic energy of charged particles into visible light. Gamma rays that interact with a scintillator will produce secondary

electrons, for example through photoelectric absorption or Compton scattering, and 
the secondary electrons will then interact with the structure of the scintillator and produce scintillation light. The amount of scintillation light that is produced can be related to the energy deposited by the secondary electrons, allowing for measurements of the scintillation light to serve as an appropriate measure of the energy deposited in the detector. Scintillation light is converted to an electronic signal for analysis by a photodetector.

There are two general classes of scintillation materials: inorganic and organic. ${ }^{22}$ The scintillation process in organic scintillators arises from energy-level transitions in single molecules within the material, whereas the mechanism in inorganic scintillators originates from the crystal lattice of the material. The detector utilized for the work presented in this thesis employs thallium-doped cesium iodide, $\mathrm{CsI}(\mathrm{Tl})$, which is an inorganic scintillator. This section provides more details about scintillation detectors, the scintillation mechanism of inorganic scintillators, as well as some discussion of the properties of $\mathrm{CsI}(\mathrm{Tl})$ as a scintillator.

\subsubsection{Energy spectrum in gamma-ray spectroscopy}

Figure 2.7 shows a schematic illustration of the theoretical energy spectrum that would be obtained in a scintillation detector of intermediate volume and perfect energy resolution when measuring a gamma-ray source that emits monoenergetic photons of energy $E_{\gamma}$, where "intermediate volume" is defined in such a way that a gamma ray may undergo more than one interaction within the volume of the scintillator but does not necessarily deposit all of its energy within the scintillator. Neither subfigure is to scale. Figure 2.7a illustrates the spectrum that would be expected when $E_{\gamma}$ is less than the threshold energy of $2 m_{\mathrm{e}} c^{2}$ for pair production. The photopeak at $E_{\gamma}$ has been indicated, which for perfect energy resolution would have no width; however, for the purposes of visualization, it has been represented in figure 2.7 a with a small finite width. Other notable features that have been indicated include the Compton edge, the Compton valley, and the Compton continuum. The photopeak occurs when a gamma ray deposits all of its energy within the scintillator. The Compton edge is defined by the maximum energy which can be deposited by a gamma ray that undergoes a single Compton scattering interaction, which occurs when it scatters through $180^{\circ}$ within the volume of the scintillator and departs with no further interaction. The Compton 
continuum is made up from gamma rays which undergo Compton interactions of all different scattering angles within the volume of the scintillator; gamma rays that undergo multiple interactions before escaping from the scintillator may deposit energies in excess of the Compton edge, populating counts in the Compton valley.

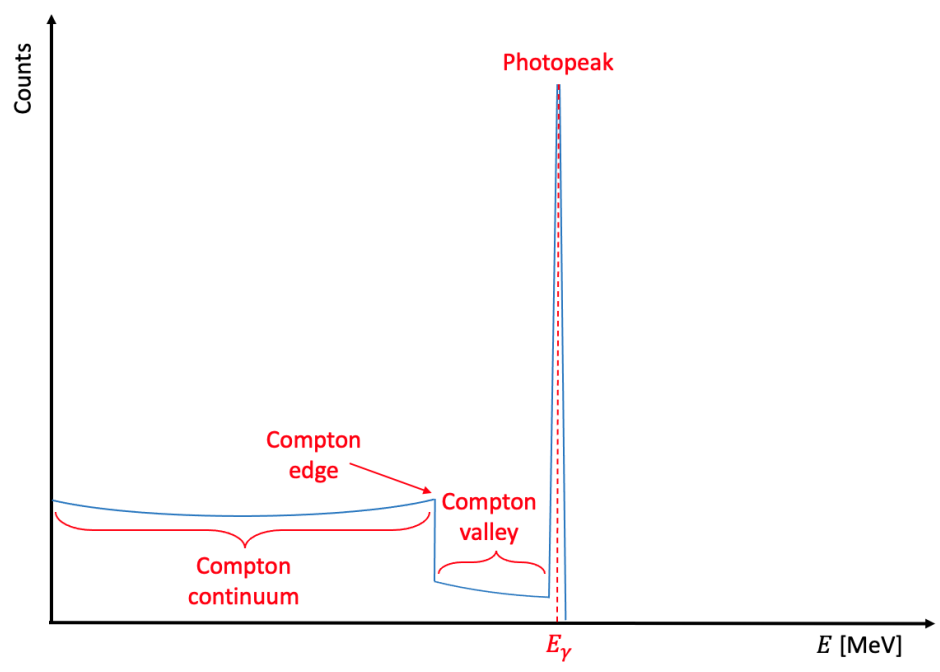

(a)

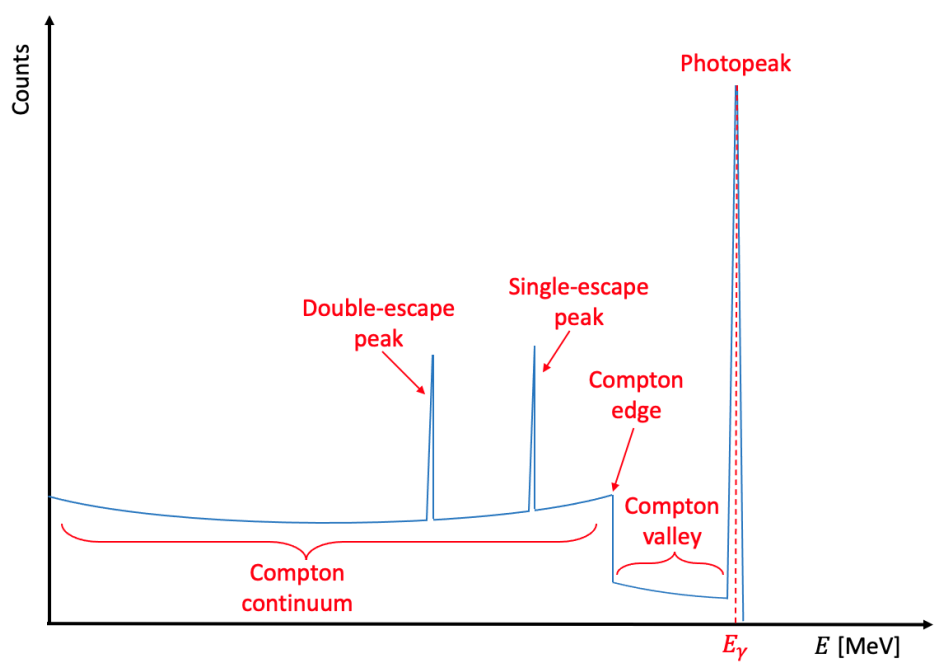

(b)

Figure 2.7: Schematic of theoretical energy spectrum for a scintillator of intermediate size measuring monoenergetic gamma rays of energy $E_{\gamma}$. a) Spectrum when $E_{\gamma}<2 m_{\mathrm{e}} c^{2}$. b) Spectrum when $E_{\gamma}>2 m_{\mathrm{e}} c^{2}$. In both sub-figures, peaks have been represented with finite width for the purposes of visualization. Neither sub-figure is to scale. 
Figure 2.7b illustrates the spectrum that would be expected when $E_{\gamma}$ is larger than $2 m_{\mathrm{e}} c^{2}$. The features described for figure 2.7 a remain, but the possibility of pair-production inside the scintillator introduces single- and double-escape peaks. A double-escape peak occurs when a pair-production interaction takes place within the volume of the scintillator and both of the $511 \mathrm{keV}$ photons that result from interaction of the produced positron with an atomic electron escape the scintillator without further interaction, resulting in a peak displaced $1022 \mathrm{keV}$ from the photopeak. The single-escape peak occurs when only one of the $511 \mathrm{keV}$ photons escapes, resulting in a peak displaced $511 \mathrm{keV}$ from the photopeak.

The escape of characteristic $\mathrm{x}$ rays from the detector may give rise to an $\mathrm{x}$-ray escape peak, which is not pictured in figure 2.7 but can occur in either of the energy regimes described above. Characteristic x rays are produced following a photoelectric absorption event when an atomic electron from a higher energy level than the one that was ejected drops to the lower energy level to fill the vacancy, emitting the energy difference in the form of an $\mathrm{x}$ ray. If the resulting $\mathrm{x}$ ray has energy $E_{X}$ and escapes from the detector without further interaction, then the x-ray escape peak would occur on the energy scale at $E_{\gamma}-E_{X}$.

In real detectors, lack of perfect energy resolution results in a broadening of the peaks in the spectrum. The binding energy of the electron also results in the introduction of a finite slope to the Compton edge, rather than a sharp cut-off. The presence of noise may additionally affect the shape of the spectrum, especially at lower energies. Finally, scattering of gamma rays from materials in the vicinity of the detector can introduce a back-scatter peak and x-ray peaks due to characteristic x-ray emissions from the nearby materials. Examples of experimentally measured energy spectra for $\mathrm{CsI}(\mathrm{Tl})$ will be shown in chapter 3 .

\subsubsection{Energy resolution}

The energy resolution $R\left(E_{\gamma}\right)$ of a scintillation detector as a function of energy $E_{\gamma}$ is defined by

$$
R\left(E_{\gamma}\right)=\frac{\operatorname{FWHM}\left(E_{\gamma}\right)}{E_{\gamma}} \times 100 \%
$$

where FWHM $\left(E_{\gamma}\right)$ is the full-width at half-maximum of a photopeak that would be measured for a source emitting photons of energy $E_{\gamma} \cdot{ }^{22}$ In practice, a scintillation 
detector is composed not only of a scintillator but also contains a photodetector and readout electronics. The overall energy resolution $R\left(E_{\gamma}\right)$ is obtained by a quadrature sum of the resolutions of each component of the detector. In general, the energy resolution for a scintillation detector may be approximated as

$$
R^{2}\left(E_{\gamma}\right)=R_{\text {scint }}^{2}\left(E_{\gamma}\right)+R_{\text {phot }}^{2}\left(E_{\gamma}\right)+R_{\text {elec }}^{2}\left(E_{\gamma}\right)
$$

where $R_{\text {scint }}\left(E_{\gamma}\right)$ is the energy resolution which can be ascribed to the scintillator, $R_{\text {phot }}\left(E_{\gamma}\right)$ is the energy resolution of the photodetector, and $R_{\text {elec }}\left(E_{\gamma}\right)$ is the resolution component which can be attributed to the readout electronics.

\subsubsection{Inorganic scintillators}

The scintillation process in an inorganic scintillator arises from the transition of secondary electrons through the energy states available within the crystal lattice. ${ }^{22}$ Figure 2.8a illustrates the energy band structure of a pure inorganic scintillator, in which the vertical axis represents the increasing energy levels in the band structure. Electrons bound to atoms in the lattice occupy the lower available energy bands, with the highest energy of these bands being referred to as the valence band. Electrons that obtain sufficient energy can be excited to the conduction band, which is the next energy band after the valence band; electrons in the conduction band are those which have been liberated from atomic binding and may move throughout the crystal. The energy required to excite an electron from the valence band to the conduction band is referred to as the band gap. In a pure crystal, such as that illustrated in figure 2.8a, the de-excitation of electrons from the conduction band back to the valence band is inefficient, the probability of photon emission with such an electron transition is low, and photons that are released have too high of an energy to be reliably detected by standard photodetectors. ${ }^{22}$

To produce more efficient inorganic scintillators, the material is altered to introduce intermediate energy levels inside the band gap in which electrons cannot normally exist in the pure material. This is accomplished by adding impurities to the lattice, known as activators or dopants, which replace atoms of the original material in the crystal lattice. Inorganic scintillators that have had activators added to them are referred to as being doped. Figure $2.8 \mathrm{~b}$ illustrates the energy band structure of a doped inorganic scintillator. The addition of the activator introduces intermediate 
energy states in the band gap, depicted as dashed blue lines. The additional activator energy states allow efficient de-excitation of electrons from the conduction band to the valence band. As the electron de-excites through the energy levels made available by the activator, visible photons, illustrated by a red wavy line, are released that are subsequently measured as part of the scintillation signal.

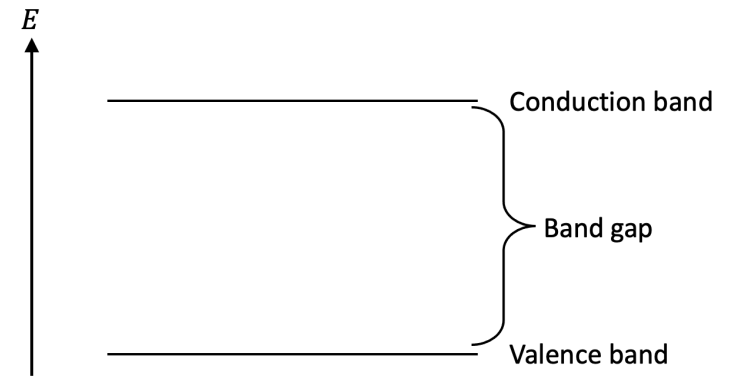

(a)

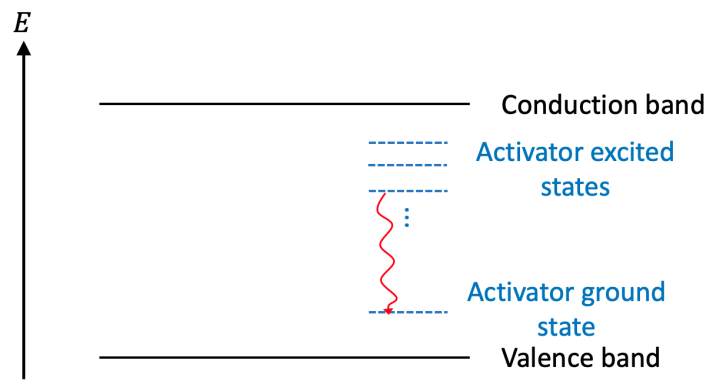

(b)

Figure 2.8: Illustration of the energy band structure for an inorganic scintillator. a) Energy band structure for pure crystalline lattice. b) Energy band structure for a crystalline lattice with activator impurities added. Transitions between activator energy states release visible light photons, illustrated by a wavy red line. The energy $E$ of the bands increases along the vertical axis in both subfigures.

\subsection{4 $\mathrm{CsI}(\mathrm{Tl})$}

CsI is an alkali halide with a density of $4.51 \mathrm{~g} / \mathrm{cm}^{3}$ that is relatively soft, does not cleave readily under mechanical shock, and is only mildly hygroscopic. ${ }^{22,23,32}$ This makes it well suited for use in gamma-ray detectors which may operate in outdoor conditions.

CsI is frequently doped with thallium, producing $\mathrm{CsI}(\mathrm{Tl})$. The concentration of $\mathrm{Tl}$ in $\mathrm{CsI}(\mathrm{Tl})$ varies, but is typically several hundred to several thousand parts per million. ${ }^{33} \mathrm{CsI}(\mathrm{Tl})$ has a light yield of approximately 40,000 to 67,000 scintillation photons per $\mathrm{MeV}$ of energy deposited. ${ }^{32,34,35}$ Figure 2.9 shows the emission spectrum of $\mathrm{CsI}(\mathrm{Tl})$, generated from data provided in reference 32. The peak emission wavelength occurs at approximately $550 \mathrm{~nm}$, green in colour. The decay of the scintillation output from $\mathrm{CsI}(\mathrm{Tl})$ consists of two components, one with a $3.5 \mu$ s decay time and 
one with a $0.6 \mu$ s decay time. The average primary decay time may be taken as $1 \mu \mathrm{s}$ for $\operatorname{CsI}(\mathrm{Tl})$ for gamma rays. ${ }^{32}$

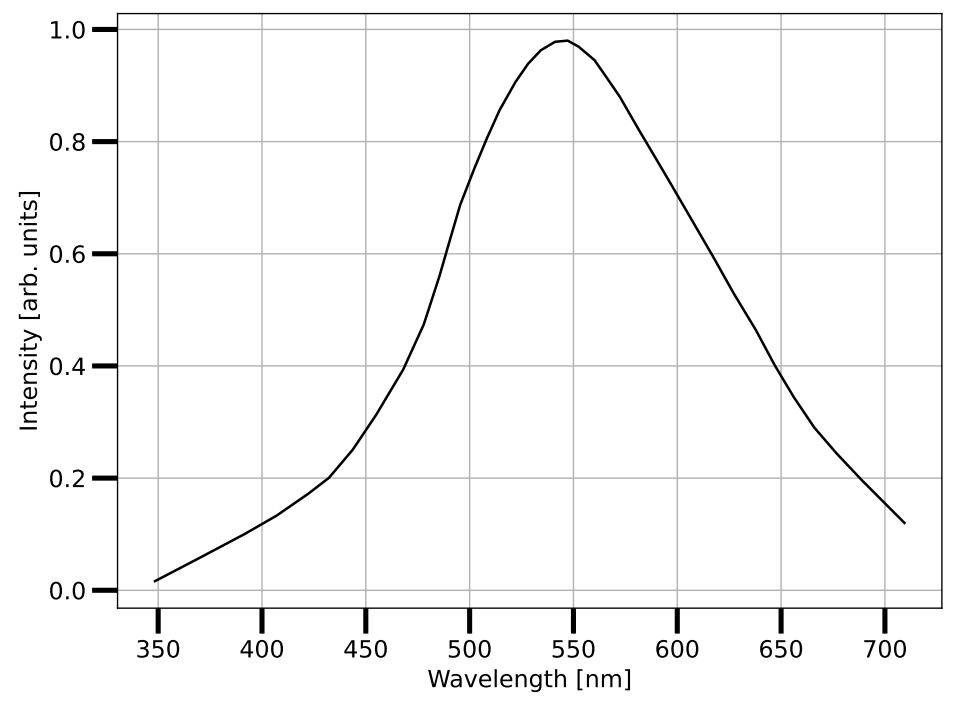

Figure 2.9: Scintillation emission spectrum for CsI(Tl). This figure was generated using data provided in reference 32.

Figure 2.10 shows the temperature dependence of the scintillation light output for $\mathrm{CsI}(\mathrm{Tl})$, generated from data provided in reference 32. The relative light output of $\mathrm{CsI}(\mathrm{Tl})$ is at a maximum for temperatures between $25^{\circ} \mathrm{C}$ and $30{ }^{\circ} \mathrm{C}$, and begins to fall for temperatures outside of this range; at a temperature of $-10{ }^{\circ} \mathrm{C}$, the relative light output of $\mathrm{CsI}(\mathrm{Tl})$ is approximately $88 \%$ of the maximum. This has an effect on measurements taken by a detector that uses $\mathrm{CsI}(\mathrm{Tl})$, such as the detector utilized for the work presented in this thesis, and must be accounted for.

Measurements with large-area avalanche photodiodes optimized to the scintillator being measured have determined that the energy resolution of $\mathrm{CsI}(\mathrm{Tl})$ is between $4.8 \%^{36}$ and $4.9 \%^{35}$ at $662 \mathrm{keV}$. 


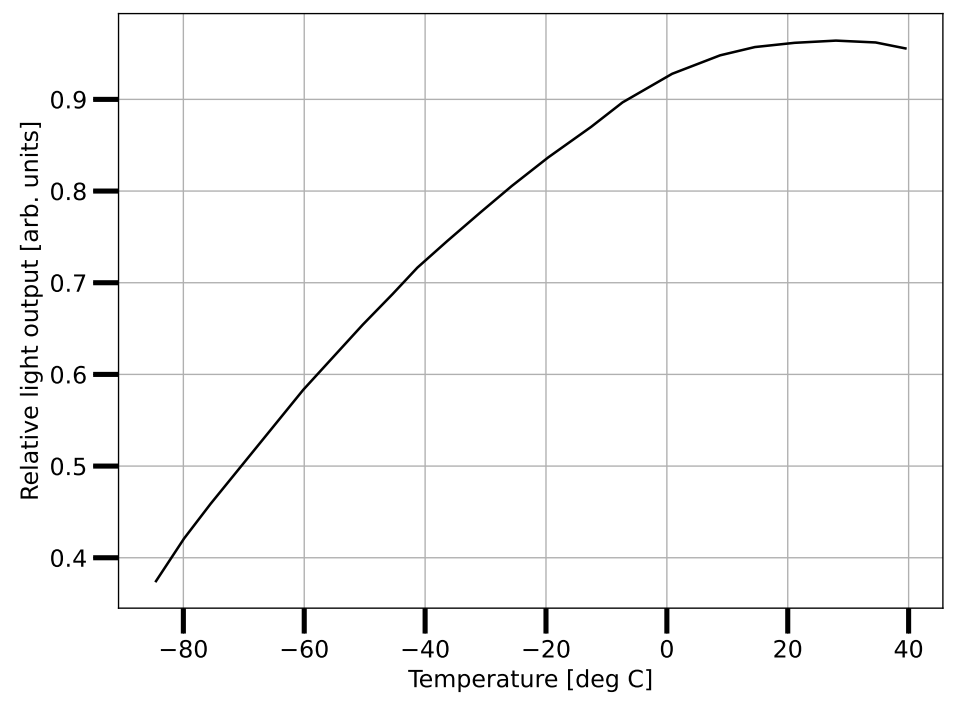

Figure 2.10: Temperature dependence of light output in CsI(Tl). This figure was generated using data provided in reference 32.

\subsection{Silicon photomultipliers}

Silicon photomultipliers (SiPMs) are a modern alternative to the conventional photomultiplier tube. Their low-voltage requirements, small profile, and mechanical robustness make them well-suited to applications in outdoor operational scenarios. The detector used for the work presented in this thesis employs SiPMs to measure the scintillation light produced in $\mathrm{CsI}(\mathrm{Tl})$ scintillation crystals. This section briefly reviews the physics of semiconductor diodes before presenting an overview of SiPMs.

\subsection{1 p-type and n-type semiconductors}

An n-type semiconductor is one in which a doping agent containing one extra valence electron compared to the original semiconductor species is added, resulting in a surplus of electrons relative to the original lattice structure. ${ }^{22}$ An example of this is illustrated in figure 2.11, where a lattice of silicon ( $\mathrm{Si}$ ) atoms, represented by blue circles, has had a phosphorous (P) dopant added, represented by the grey circle. Each of the Si atoms has four valence electrons and forms a covalent bond with four other $\mathrm{Si}$ atoms, represented in figure 2.11 by the solid black lines. However, $\mathrm{P}$ atoms have five valence electrons, and when introduced to a lattice of Si atoms will form four covalent bonds, leaving the fifth valence electron unbound. The free electron, represented in 
figure 2.11 by the small yellow circle with dashed line, is loosely bound to the $\mathrm{P}$ atom and may drift throughout the material, contributing to an excess of negative charge carriers.

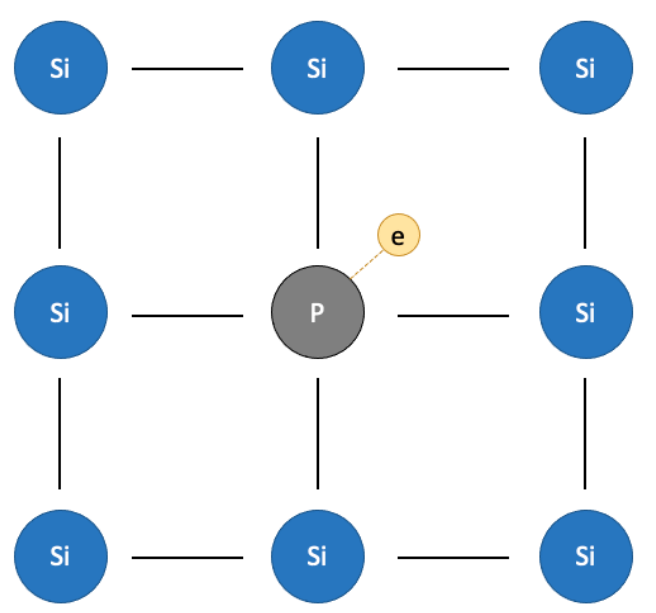

Figure 2.11: Illustration of a representative n-type material. The blue circles represent Si atoms, the grey circle represents a $P$ atom, the solid black lines represent covalent bonds, and the small yellow circle represents the surplus valence electron on $P$.

A p-type semiconductor is formed in a similar way to an n-type semiconductor, except the dopant that is added to the sample will have one fewer valence electrons compared to the original semiconductor species. ${ }^{22}$ Figure 2.12 illustrates an example of a p-type material, where a boron (B) atom has been added to sample of $\mathrm{Si}$ atoms, illustrated as grey and blue circles respectively. Boron has only three valence electrons and can therefore form only three covalent bonds with the surrounding $\mathrm{Si}$ atoms, drawn in figure 2.12 by the solid black lines. The fourth unfilled binding site around the $\mathrm{B}$ atom contributes to an excess of positive charge carriers known as holes, represented by the white circle in figure 2.12 . 


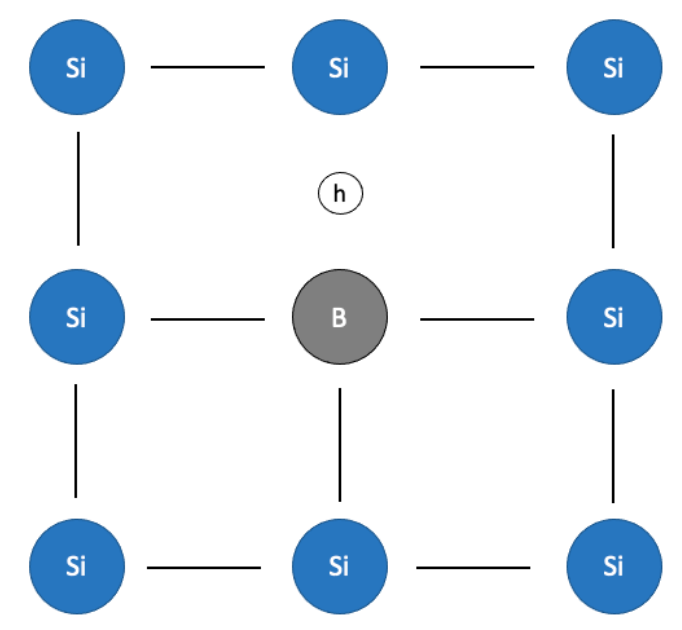

Figure 2.12: Illustration of a representative p-type material. The blue circles represent Si atoms, the grey circle represents a B atom, the solid black lines represent covalent bonds, and the small white circle represents a hole at the unbound site around the $B$ atom.

\subsubsection{Semiconductor diodes}

When a p-type material is brought into good thermodynamic contact with an ntype material, a p-n junction is created within the resulting solid. ${ }^{22}$ The gradient in electron and hole concentrations at the junction causes electrons from the n-type material to diffuse towards the p-type material, while holes from the p-type material diffuse towards the n-type material. As electrons from the n-type material diffuse into the p-type material, the ionized donor impurities left behind in the n-type material near the junction contribute to a positive space charge. Similarly, as holes from the p-type material diffuse into the n-type material, the acceptor impurities left behind in the p-type material near the junction acquire an excess of negative charge. The diffusion process reaches a steady-state equilibrium when the space charges on either side of the junction builds up an electric field strong enough to stop the net diffusion of charge carriers across the junction. The region at the junction of the two materials where the diffusion of charge carriers is balanced is referred to as the depletion region. This is illustrated in figure $2.13 \mathrm{a}$, which shows a p-type material in blue connected to an n-type material in green, with the depletion layer in grey at the junction. Positive holes are represented by white circles, while negative electrons are represented by yellow circles. 
An external voltage may be applied to a material with a $\mathrm{p}-\mathrm{n}$ junction to alter its properties. If the p-type material is made negative with respect to the $\mathrm{n}$-type material, the p-n junction will be reverse biased. ${ }^{22}$ This results in an extension of the depletion region, and is illustrated in figure 2.13b. A p-type material, drawn in blue, is attached to the negative terminal of an external voltage source. A n-type material, represented in green, is attached to the positive terminal of an external voltage source. Holes in the p-type material, represented by white circles, will be attracted towards the negative source, whereas electrons in the n-type material, represented by yellow circles, will be attracted towards the positive source. The depletion region, illustrated in grey, is drawn wider than was illustrated in figure 2.13a for a $\mathrm{p}-\mathrm{n}$ junction with no biasing applied.

\begin{tabular}{|c|c|c|c|c|c|c|c|c|}
\hline \multirow{2}{*}{\multicolumn{9}{|c|}{ depletion region }} \\
\hline & & & & & 7 & n-typ & & \\
\hline \multirow{4}{*}{$\begin{array}{c}\oplus \\
\oplus\end{array}$} & & & $\oplus$ & $\odot$ & $\stackrel{+}{\oplus}$ & $\odot$ & $\Omega$ & $\odot$ \\
\hline & $A$ & ๑ & & $\odot$ & ( & $\odot \odot$ & & \\
\hline & & & (†) & $\odot$ & $\oplus$ & $\odot$ & 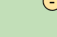 & \\
\hline & † & & $\oplus$ & $\odot$ & ๑ & $\odot \quad \odot$ & $\odot$ & $\odot$ \\
\hline
\end{tabular}

(a)

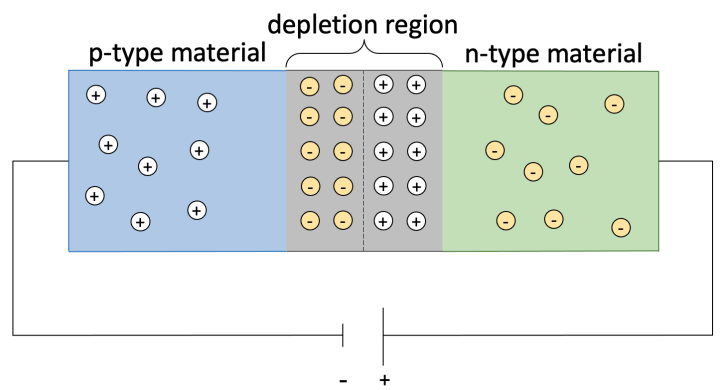

(b)

Figure 2.13: Illustration of semiconductor junctions for unbiased and reverse-biased diodes. a) Unbiased diode. b) Reverse-biased diode. In both subfigures, the p-type material is represented in blue, the n-type material in green, the depletion region at the junction in grey, positive holes by white circles, and negative electrons by yellow circles.

\subsubsection{Single-photon avalanche diodes}

If a silicon diode is operated under a sufficiently high reverse bias, electron-hole pairs liberated in the material by incident photons will be accelerated with sufficient kinetic energy to liberate secondary charged particles by impact ionization. The voltage after which this phenomena occurs is called the breakdown voltage, $V_{\mathrm{bd}}$. Impact ionization results in a cascade of ionizations throughout the silicon, which amplifies a single electron-hole pair liberated by one photon into a measurable macroscopic flow. The 
process forming the ionization cascade is known as Geiger discharge, and a silicon diode operated in this Geiger mode is known as a single-photon avalanche diode (SPAD). ${ }^{37}$ The cascade of ionization is sometimes referred to as a Geiger avalanche.

If left unchecked, the Geiger avalanche in the SPAD can damage the material. To stop this from happening, a series resistor referred to as the quenching resistor is used to limit the current which can be drawn by the SPAD when in a Geiger mode breakdown. This has the effect of lowering the magnitude of the reverse bias at the diode below the breakdown voltage, reducing the kinetic energy of the charge-carrier pairs in the material and stopping the flow of current in the diode. After a short period, the SPAD will recharge to the normal reverse bias voltage and will be able to undergo another cascade when initiated by an incident photon. The time that it takes the SPAD to recharge is much less than the average decay time of scintillation output from $\mathrm{CsI}(\mathrm{Tl})$.

Although SPADs produce measurable electronic signals from photon interactions, they are binary in nature. The number of photons interacting within the volume of the silicon does not influence the magnitude of the electronic signal once the SPAD begins conducting electricity, and a new signal is not measurable until the SPAD has undergone a quenching and recharging cycle.

\subsubsection{SiPMs}

The combination of a single SPAD and its associated quenching resistor is referred to as a microcell. An SiPM is a photodetector made from a dense array of independently operating microcells, typically a few to several $\mathrm{mm}$ in size. The electronic signals generated from each of the microcells are summed together in the SiPM to provide a signal that is proportional to the incident photon flux.

Typical microcell densities on an SiPM are ${ }^{37}$ between $100 \mathrm{~mm}^{-2}$ and several $1000 \mathrm{~mm}^{-2}$. The density of microcells on the surface of an SiPM is quantified by the fill factor, which is the percentage of the surface of the SiPM which is covered by the active face of the SPADs. Each microcell necessarily introduces some inactive area to the SiPM due to the need to provide electronic and optical isolation from its neighbours. Larger microcells result in a higher fill factor, but at the expense of having fewer microcells on the SiPM. A higher fill factor is desirable because it avoids 
loss of optical photons in the dead material between SPADs, which improves energy resolution. However, there must also be a sufficient number of microcells on the $\mathrm{SiPM}$ in order to provide a sufficient dynamic range with respect to energy. Too few microcells would result in the SiPM producing a constant signal above some threshold energy at which there are enough scintillation photons produced to trigger all of the microcells.

An SiPM is operated at a bias that is slightly higher than the breakdown voltage of the SPADs in the microcells. The difference between the breakdown voltage and the operating voltage, $V_{\text {bias }}$, is known as the overvoltage, $V_{\text {over }}$ :

$$
V_{\mathrm{bias}}=V_{\mathrm{bd}}+V_{\text {over }}
$$

Typical values of $V_{\mathrm{bd}}$ in a silicon SPAD may be around $25 \mathrm{~V}$, and an overvoltage $10 \%$ to $25 \%$ higher than the breakdown voltage is typically used when operating an SiPM. ${ }^{37}$

The gain of an SiPM, $G_{\mathrm{SiPM}}$, corresponds to the amount of charge created for each detected photon and is proportional to the value of $V_{\text {over }}:{ }^{37}$

$$
G_{\mathrm{SiPM}}=\frac{C_{\mathrm{mc}} V_{\mathrm{over}}}{q_{\mathrm{e}}}
$$

where $C_{\mathrm{mc}}$ is the capacitance of the microcell and $q_{\mathrm{e}}$ is the charge of an electron.

The photon detection efficiency is a quantitative measure of the sensitivity of an SiPM to photons of different wavelengths. The photon detection efficiency increases as $V_{\text {over }}$ is made larger. This is because larger values of $V_{\text {over }}$ increase the avalanche initiation probability within a SPAD. Figure 2.14 shows the photon detection efficiency for the model of SiPM used in the detector that made the measurements for this thesis, operated at an overvoltage of $5 \mathrm{~V}$. Figure 2.14 was generated using data provided in reference 38. The relationship between the peak emission of the $\mathrm{CsI}(\mathrm{Tl})$ shown in figure 2.9 and the photon detection efficiency for an SiPM shown in figure 2.14 will be discussed in more detail within chapter 3 .

Even in the absence of an incident photon fluence, a signal will be measured from an SiPM. This is known as the dark-count rate, and is primarily due to thermal electrons generated in the active material. The dark-count rate is the main source of noise in an SiPM. ${ }^{37}$ Thermal electrons will initiate a Geiger avalanche that is 


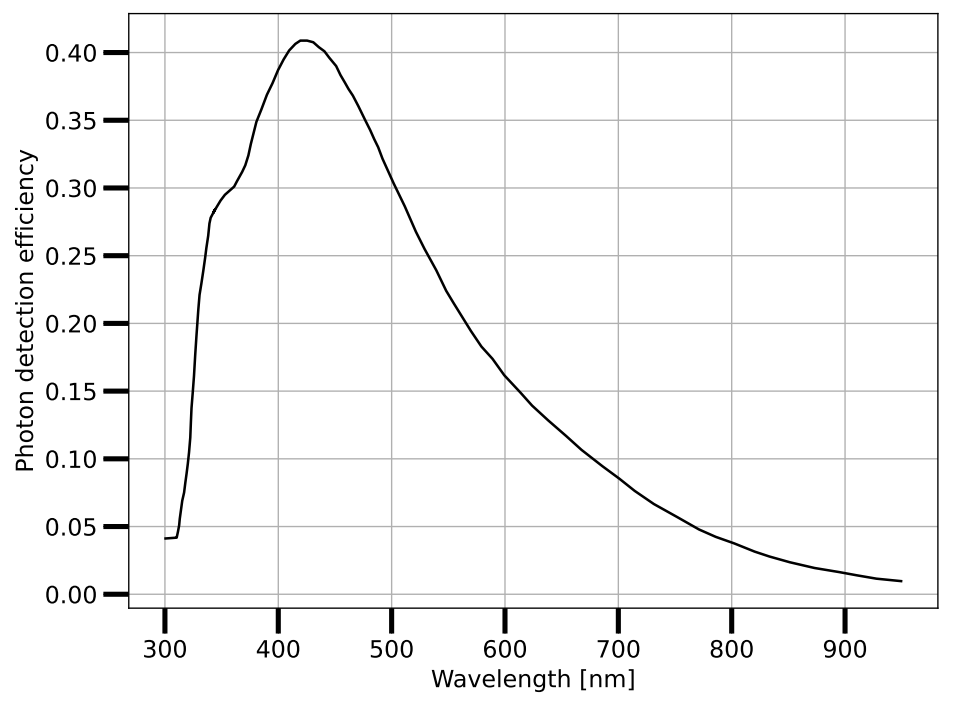

Figure 2.14: Photon detection efficiency for an SiPM operated at an overvoltage of $5 \mathrm{~V}$. This figure was generated using data provided in reference 38.

indistinguishable from one initiated by a photon interacting in the material. Setting a trigger threshold above the single-photon level assists in reducing the number of false triggers due to dark-count events, though the dark-count rate will still contribute to the overall measured signal. The dark-count rate depends on the active area of the SiPM, the overvoltage at which it is operated, and the temperature of the material. The dark-count rate can be reported as a rate (e.g. kCounts/s), or as an areanormalized rate (e.g. kCount/s per $\mathrm{mm}^{2}$ ). Figure 2.15 shows the dependence of the dark-count rate on the overvoltage applied to an SiPM of the type used in the detector for the work presented in this thesis; the dark-count rate increases linearly with increasing overvoltage. Figure 2.16 shows the dependence of the dark-count rate on the temperature of an SiPM of the type used in the detector for the work presented in this thesis, for an SiPM operated at a bias of $29.5 \mathrm{~V}$. Each $10{ }^{\circ} \mathrm{C}$ increase in the temperature results in a 50\% increase in the dark-count rate. Chapter 3 will discuss the choice of operating voltage for the detector used for the work presented in this thesis, and chapter 4 will discuss how temperature effects of the combined $\mathrm{CsI}(\mathrm{Tl})$ and SiPM detector elements are mitigated. 


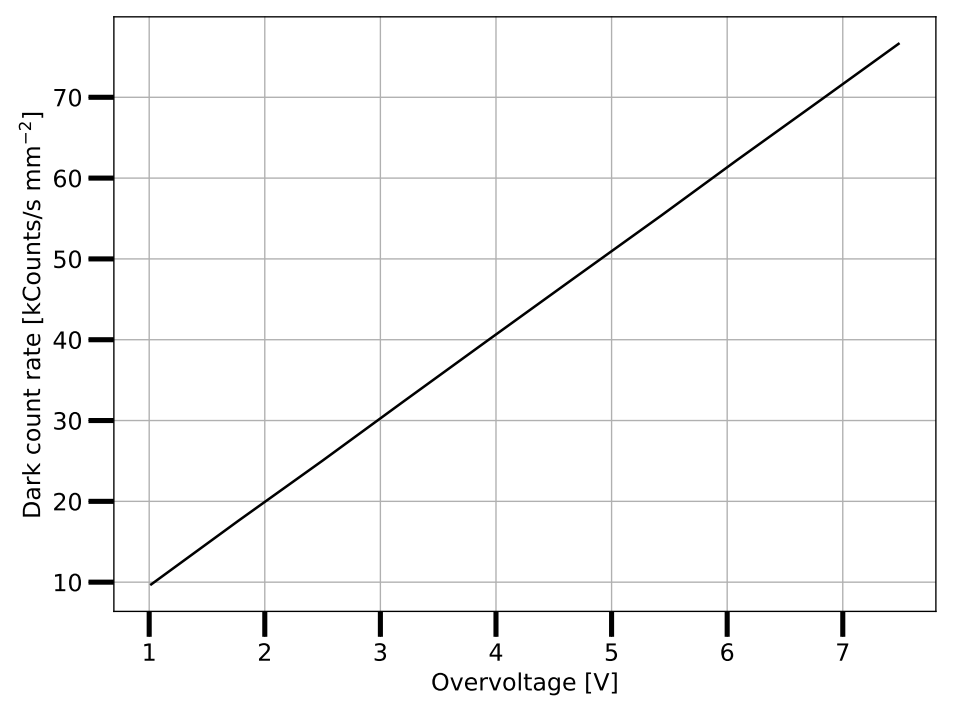

Figure 2.15: Dependence of SiPM dark-count rate on overvoltage. This figure was generated using data provided in reference 38.

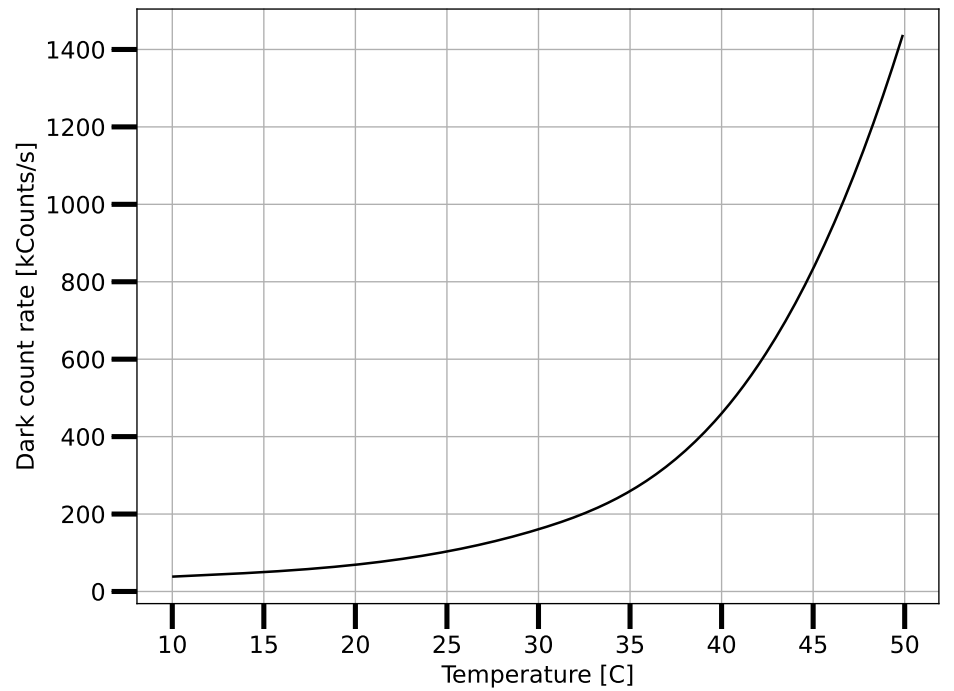

Figure 2.16: Dependence of SiPM dark-count rate on temperature for an SiPM operated at a bias of $29.5 \mathrm{~V}$. This figure was generated using data provided in reference 37. 


\subsection{Natural background radioactivity}

For any radiation measurement, the naturally occurring radioactive materials (NORM) that contribute to a background signal must be accounted for. The primary contributors to the NORM background are radionuclides in the U-238, Th-232, and K-40 series. ${ }^{39-42}$ Figure 2.17 shows the U-238 decay series, figure 2.18 shows the Th-232 decay series, and figure 2.19 shows the decay modes available to K-40, each with the half-lives of each intermediate radioisotope and the associated decays where applicable.

Not all gamma emissions occurring within the various stages of each NORM decay series occur with equal probability and some appear more prominently in gamma spectroscopy studies. Experimentally measured spectra from each of the NORM contributors using a $\mathrm{NaI}(\mathrm{Tl})$ detector, an inorganic scintillator with performance similar to $\mathrm{CsI}(\mathrm{Tl})$, are presented below.

Figure 2.20a shows the relative intensity of some gamma emissions associated with the U-238 decay series, presented as a line spectrum. Several gamma-ray emissions are illustrated, and some have been annotated with the radioisotope in the decay series that emits them along with the specific energy of the emission. Figure 2.20b shows an experimentally measured spectrum resulting from the U-238 decay series. The finite energy resolution of the detector has caused smearing of the line spectrum shown in figure 2.20a, but some peaks, such as those at $0.609 \mathrm{MeV}$ and at $1.765 \mathrm{MeV}$, remain visible. Figure 2.21a shows a line spectrum for Th-232 similar to that which was presented for U-238 in figure 2.20a, and figure 2.21b shows an experimentally measured spectrum resulting from the Th-232 decay series. The energy resolution of the detector once again results in smearing of the line spectrum presented in figure 2.21a, but peaks at e.g. $0.911 \mathrm{MeV}$ and at $2.614 \mathrm{MeV}$ remain visible. Figure 2.22a and figure $2.22 \mathrm{~b}$ show a line spectrum for K-40 and an experimentally measured spectrum from K-40, respectively. The simpler decay scheme of K-40 results in a single prominent peak at $1.460 \mathrm{MeV}$. A comprehensive catalogue of gamma-ray spectra and the emissions associated with some of the radioisotopes in the different decay series present in the NORM background may be found in references 44 and 45 .

Although figures 2.20, 2.21, and 2.22 present the spectra associated with each 


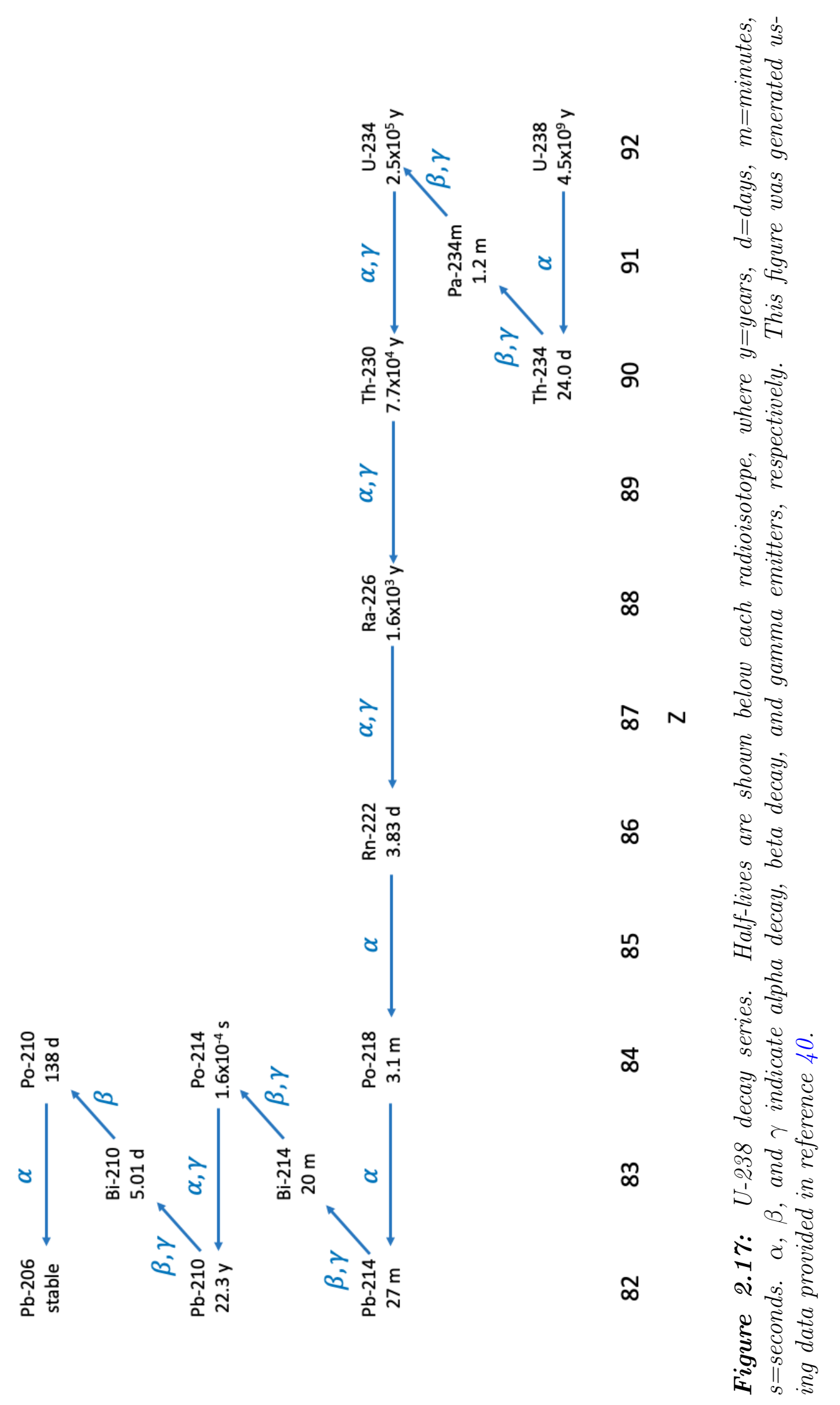




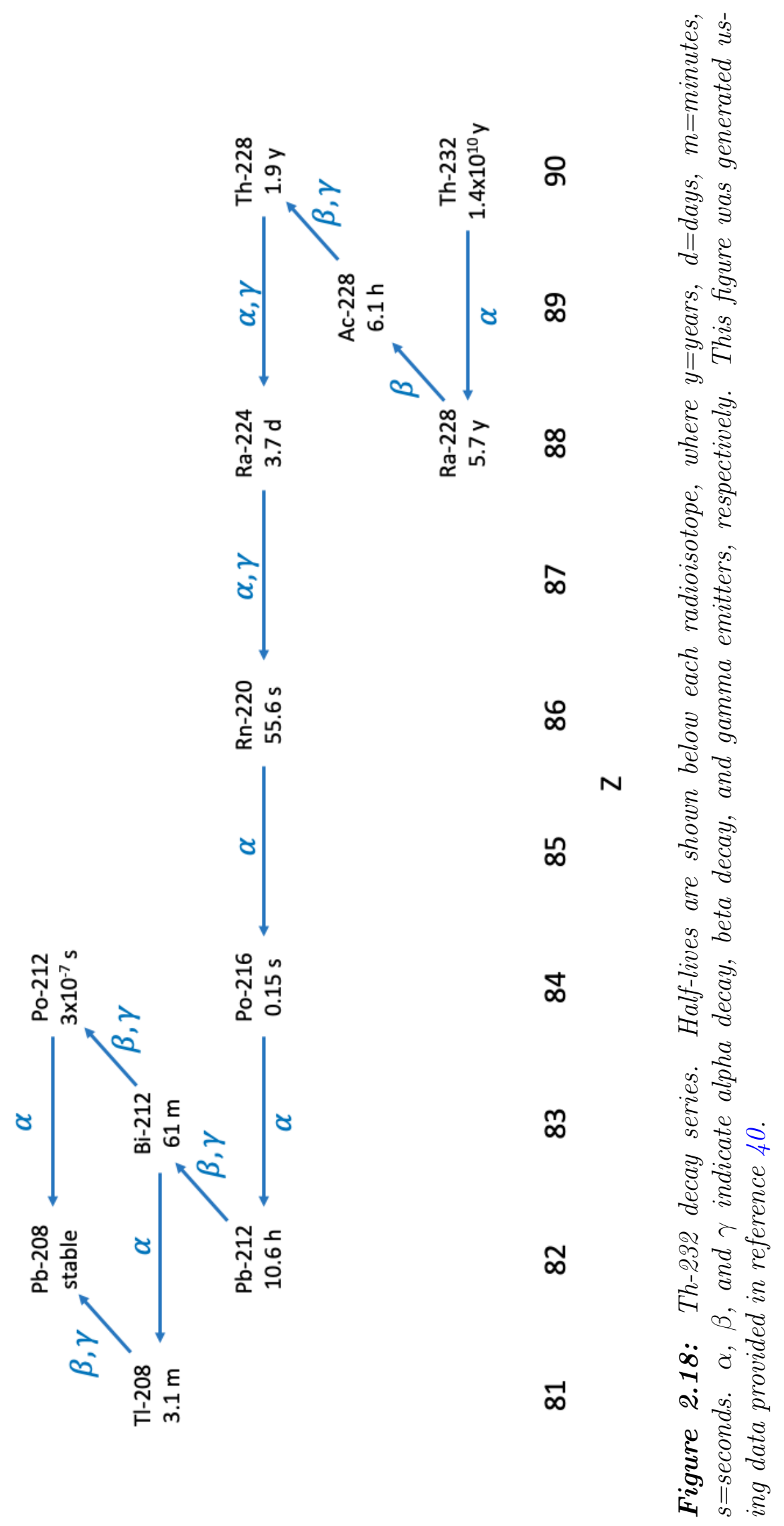




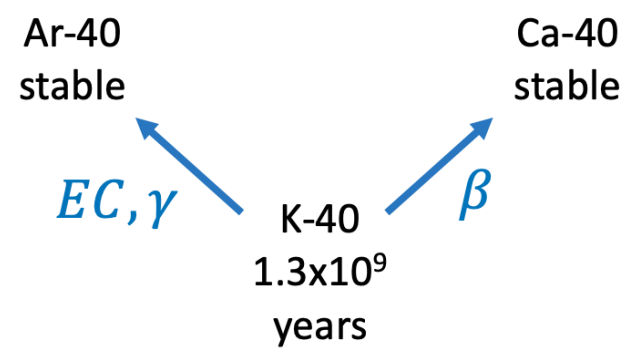

$18 \quad 19 \quad 20$

Figure 2.19: $K-40$ decay series. EC, $\beta$, and $\gamma$ indicate electron capture, beta decay, and gamma emission, respectively. This figure was generated using data provided in reference 40 .

of the individual parent radioisotopes that contribute to the NORM background, in practice the measured NORM background signal contains contributions from U-238, Th-232, and K-40 all together. Figure 2.23 shows an experimentally measured NORM background spectrum using an $\mathrm{NaI}(\mathrm{Tl})$ detector. Within figure 2.23, the finite energy resolution of the detector causes some of the closely spaced emssions from each of the NORM contributors to be smeared together (e.g. the $0.583 \mathrm{MeV}$ emission from Th232 and the $0.609 \mathrm{MeV}$ emission from U-238). However, some prominent peaks can be primarily associated with one of the individual parent radioisotopes that contribute to the NORM background; ${ }^{43}$ these have been indicated within figure 2.23. 


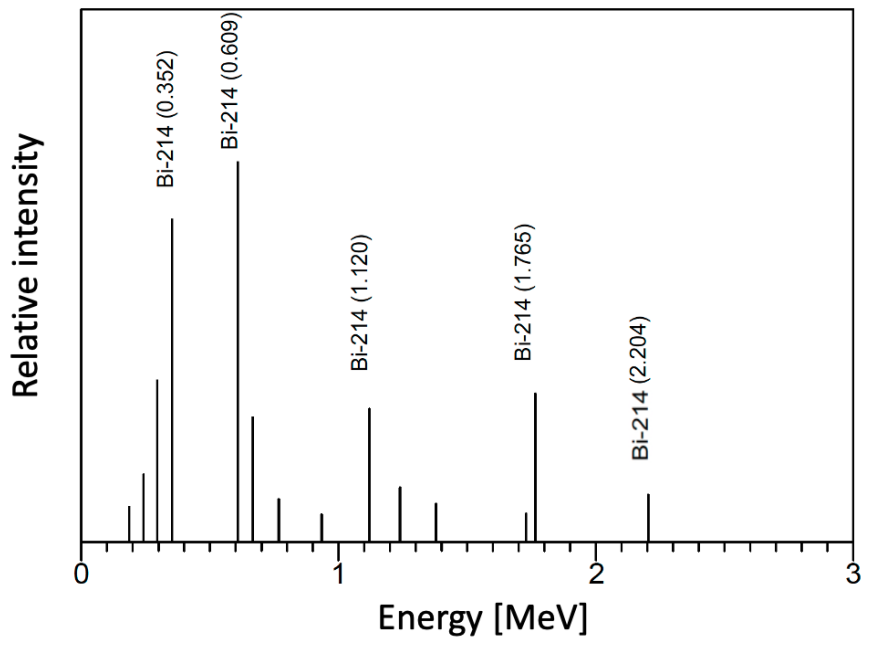

(a)

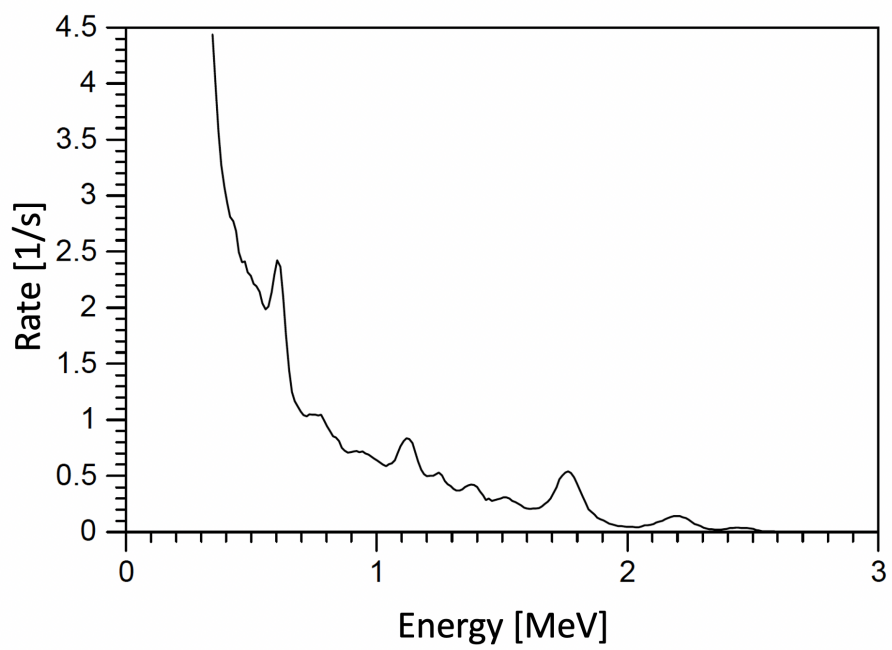

(b)

Figure 2.20: Spectrum of U-238 decay series. a) Line spectrum. b) Experimentally measured spectrum using a $\mathrm{NaI}(\mathrm{Tl})$ detector. Figures taken from reference 43. 


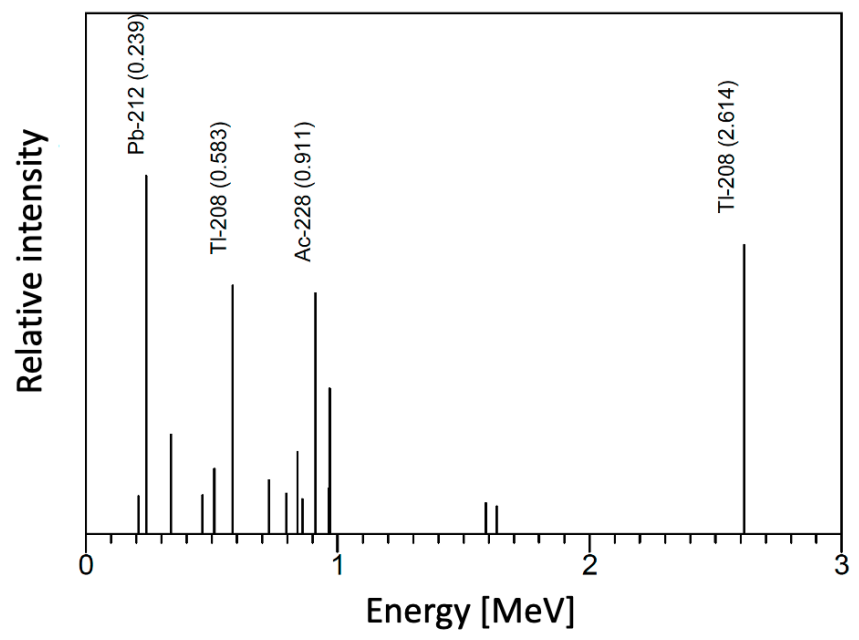

(a)

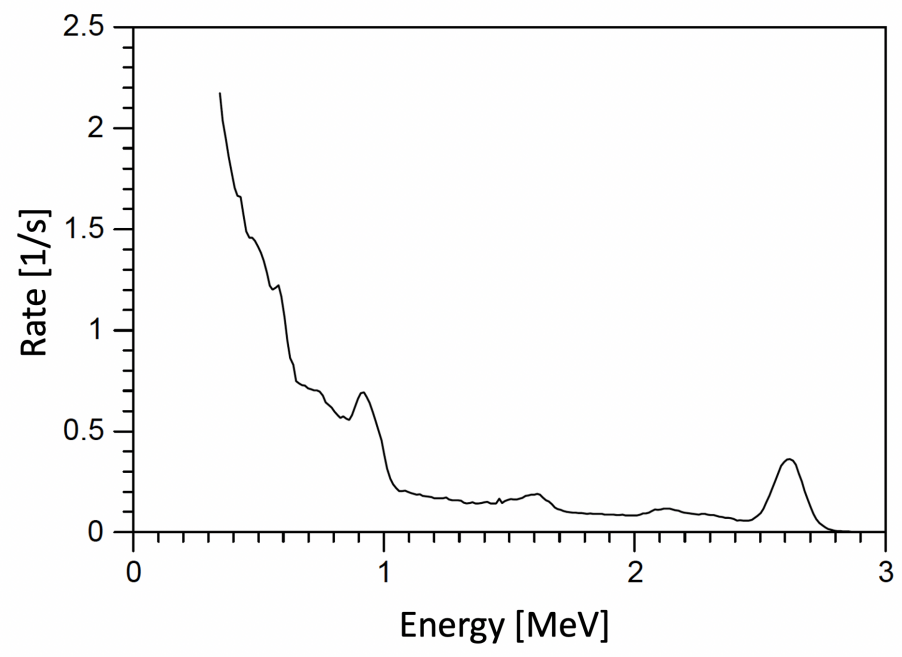

(b)

Figure 2.21: Spectrum of Th-232 decay series. a) Line spectrum. b) Experimentally measured spectrum using a NaI(Tl) detector. Figures taken from reference 43. 


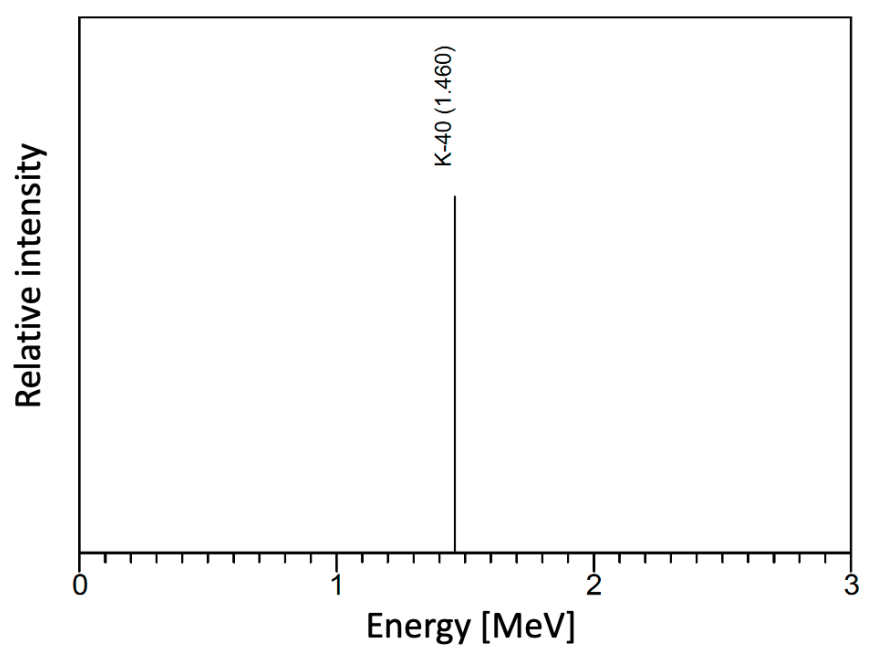

(a)

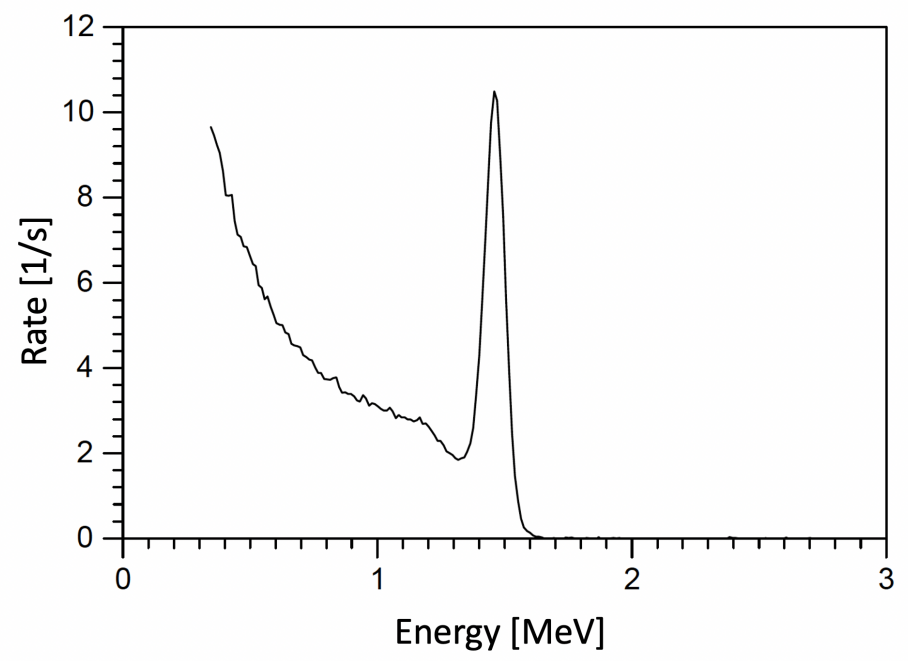

(b)

Figure 2.22: Spectrum of $K-40$ decay series. a) Line spectrum. b) Experimentally measured spectrum using a $\mathrm{NaI}(\mathrm{Tl})$ detector. Figures taken from reference 43. 


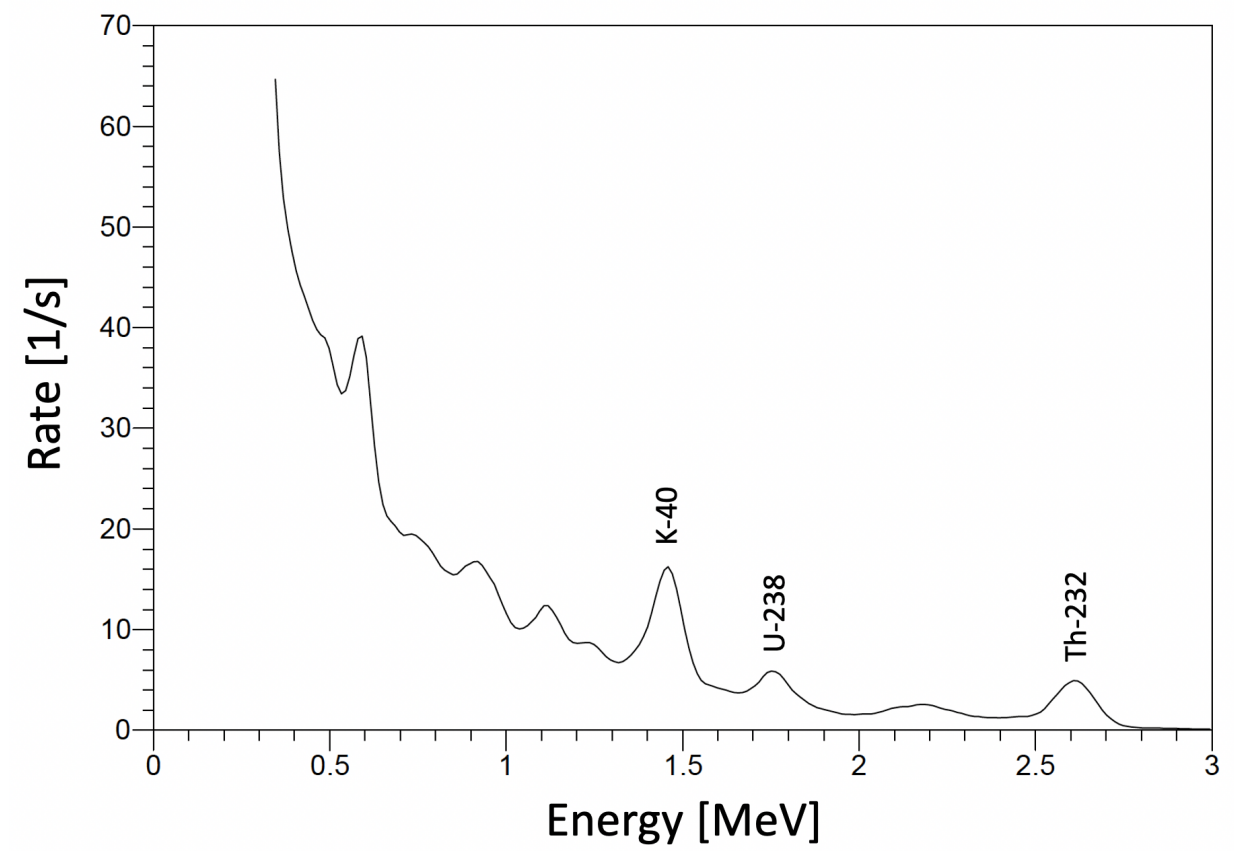

Figure 2.23: Spectrum of NORM background measured using a NaI(Tl) detector. Figure adapted from reference 43. 


\subsection{Unfolding methods}

The combined effects of limited detector resolution, instrument offsets and calibration errors, background, limited detection efficiency, and limited geometric efficiency of a radiation detector, such as a Compton gamma imager, distort the measurement relative to the true underlying signal. The process of undoing the distortions to recover the underlying signal is known as unfolding. ${ }^{22,46}$

Unfolding methods have been applied in radiological security applications in the past. Unfolding measurements of a La-140 deposited plume acquired by truckborne survey using a directional spectrometer were performed by fitting count rates acquired in each detector element. ${ }^{47} \mathrm{~A}$ method has been developed for aerial survey measurements wherein the response of an airborne gamma spectrometer to templates of radioactive materials calculated via $\mathrm{MC}$ simulation is used to recover the underlying distribution. ${ }^{48}$ This section illustrates the principles of unfolding methods in order to familiarize the reader. Methodology developed specifically for this thesis is presented in chapter 4 .

The principles of unfolding methods can be illustrated by considering the measurement of energy depositions resulting from gamma-ray interactions in a detector. $^{22,46}$ The spectrum presented in this section was generated by MC simulation of a Co-60 point source incident on the Compton gamma imager utilized for the measurements presented in this thesis; for details of the MC implementation, refer to chapter 4 . The principles of unfolding are, however, independent of the detector used to illustrate them. Co-60 was chosen for the purposes of illustration because of its simple emission spectrum.

Figure 2.24 illustrates the scenario for which the problem of unfolding a measured energy spectrum is demonstrated. A $2.5 \mathrm{MBq}$ point-source of Co-60 is represented by a blue circle and emits gamma rays represented as wavy red lines. The true emitted spectrum each second $f(E)$ is illustrated underneath the representation of the Co-60 source. The gamma rays are measured by a detector that sits $2 \mathrm{~m}$ away, represented by a grey box. Each second, the detector measures a spectrum $g\left(E^{\prime}\right)$ as illustrated underneath the representation of the detector. The relationship between $f(E)$ and $g\left(E^{\prime}\right)$ is described by the response function $R\left(E^{\prime} \mid E\right)$, which gives the probability for 
the detector to measure an energy deposition $E^{\prime}$ given that a gamma ray with energy $E$ was emitted from the source. $R\left(E^{\prime} \mid E\right)$ is described in further detail below.

Let $S\left(E^{\prime} \mid E\right)$ be the energy resolution of the detector, which describes the probability for measuring an energy $E^{\prime}$ given that a gamma ray of energy $E$ interacted within the detector, and let $\epsilon_{\operatorname{det}}(E)$ and $\epsilon_{\text {geom }}$ by the efficiency of the detector for detecting gamma rays of energy $E$ and the geometric acceptance of the detector, respectively. For this simplified discussion we will assume that there is no background, the detector is well calibrated and there are no biasing effects not contained in the resolution and efficiencies. The response function $R\left(E^{\prime} \mid E\right)$ containing all of the combined effects of $S\left(E^{\prime} \mid E\right), \epsilon_{\text {det }}(E)$, and $\epsilon_{\text {geom }}$,

$$
R\left(E^{\prime} \mid E\right)=S\left(E^{\prime} \mid E\right) \epsilon_{\mathrm{det}}(E) \epsilon_{\text {geom }}
$$

therefore contains all of the smearing effects. The relationship between $g\left(E^{\prime}\right)$ and $f(E)$ through $R\left(E^{\prime} \mid E\right)$ is: ${ }^{46}$

$$
g\left(E^{\prime}\right)=\int R\left(E^{\prime} \mid E\right) f(E) \mathrm{d} E .
$$

The action of equation 2.36 is to "fold" the response $R\left(E^{\prime} \mid E\right)$ of the detector into the measurement. Reversing the procedure is referred to as "unfolding" the response of the detector, and it allows recovery of the true underlying spectrum $f(E)$ from the measured spectrum $g\left(E^{\prime}\right)$.

Measurements of energy in a real detector are recorded in discrete windows. The discrete form of equation 2.36 is

$$
g_{i}=\sum_{j} R_{i j} f_{j}
$$

where $g_{i}$ is the number of counts of measured energy corresponding to the $i$ th window, $f_{j}$ is the intensity of emissions with true energy in the $j$ th window, and $R_{i j}$ is the entry of the response matrix that describes the probability that a measurement will be placed in the $i$ th energy window given that it had a true value occurring in the $j$ th window.

Various methods can be used to invert equation 2.37, such as attempting to invert the response matrix when it is square ${ }^{46}$ or seeking a solution for the $f_{j}$ that minimizes the residuals when compared to the measurements $g_{i}$ in a fitting procedure. ${ }^{22}$ The end result of this unfolding is an estimate of the actual emission spectrum $f(E)$. 


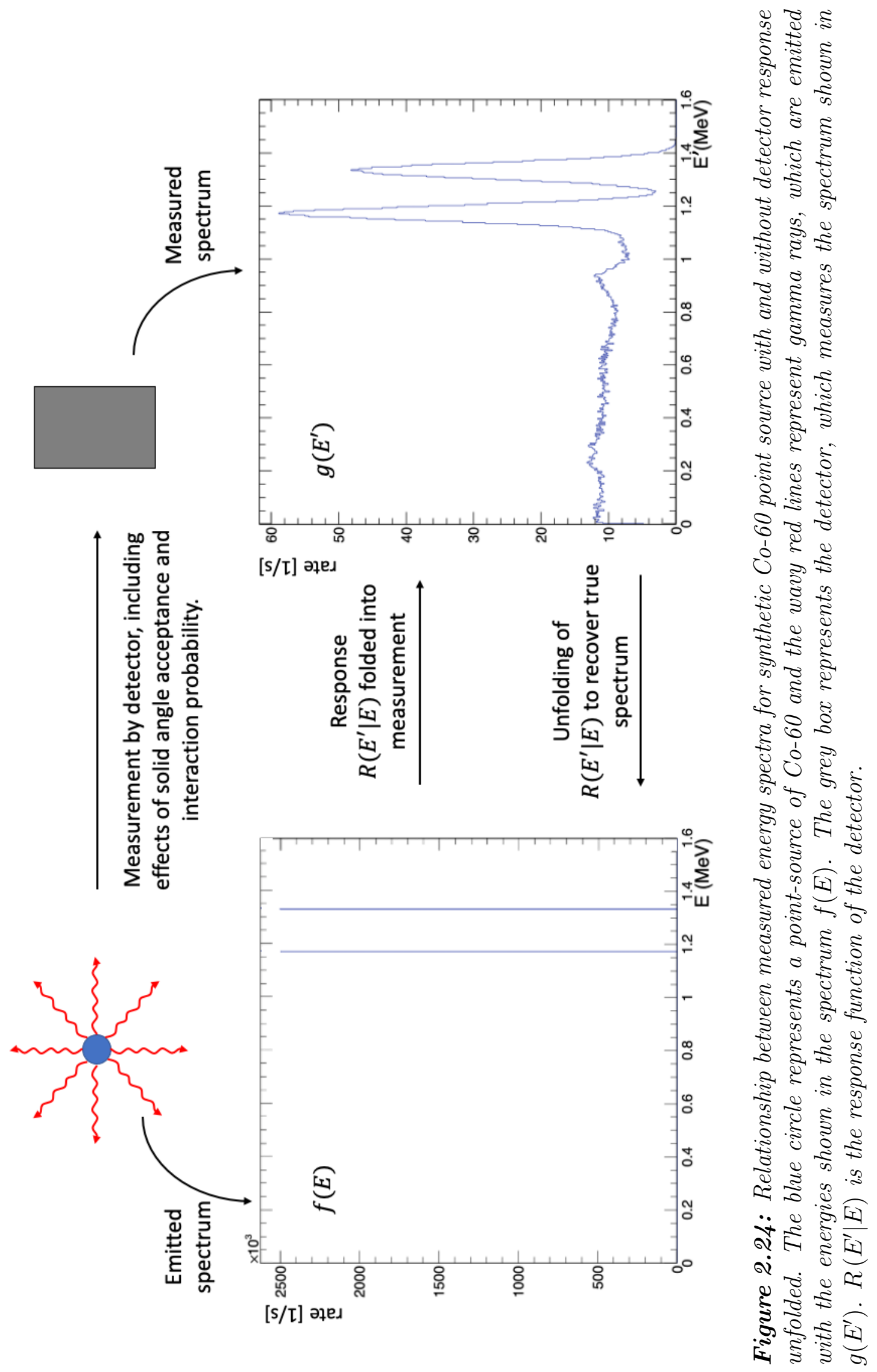




\section{Chapter 3}

\section{Instrumentation}

This chapter presents the Silicon photomultiplier-based Compton Telescope for Safety and Security (SCoTSS) detector used to collect the data for this thesis. An overview of the design of the detector is provided, before detailing some aspects of the hardware and electronics.

\subsection{The 3x3-module SCoTSS Compton gamma imager}

The SCoTSS detector uses a two-plane design, similar to that which was presented in figure 2.6 when illustrating the principles of Compton gamma imaging. Both detector planes are built from arrays of detector modules. The modular design of the SCoTSS technology allows the user to combine an appropriate number of modules for their applications, exchanging portability for sensitivity as needed. For the work presented in this thesis, a 3x3 array of modules was used in each detector plane. This configuration will be referred to as the 3x3-module SCoTSS detector. Figure 3.1 shows an interior view of the 3x3-module SCoTSS detector from the rear, where the scatter and absorber planes have been labelled and one module from each plane has been highlighted in blue.

The detector modules in each plane consist of a 4x4 array of $\mathrm{CsI}(\mathrm{Tl})$ scintillating crystals coupled to SiPMs with optical adhesive. The CsI(Tl) crystals and SiPMs are individually discussed in more detail in sections 3.2 and 3.3, respectively. Each $\mathrm{CsI}(\mathrm{Tl}) / \mathrm{SiPM}$ pair is surrounded by reflective tape to avoid loss of photons. The $4 \mathrm{x} 4$ 


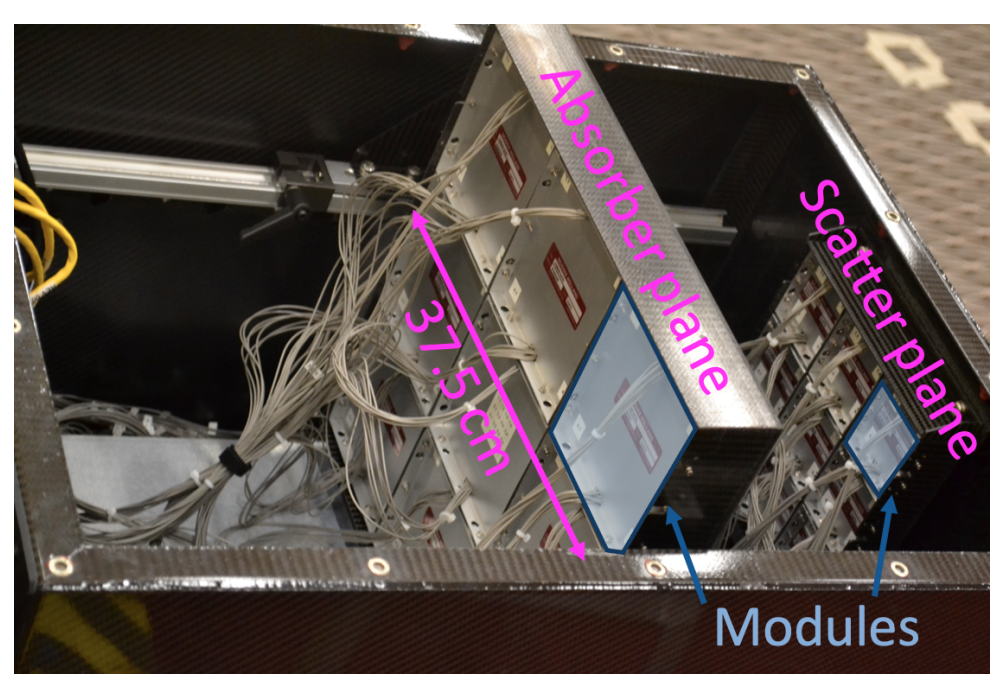

Figure 3.1: Interior view of the 3x3-module SCoTSS Compton gamma imager, with the front of the imager at the right side of the image. The detector planes have been labelled, and one module from each detector plane has been highlighted in blue.

array is then all enclosed in a layer of aluminum. This configuration provides the 3x3-module SCoTSS detector with a total of $288 \mathrm{CsI}(\mathrm{Tl}) / \mathrm{SiPM}$ pairs.

The absorber plane in the 3x3-module SCoTSS detector can be moved relative to the scatter plane. Small inter-plane distances provide lower-resolution images, whereas larger inter-plane distances provide higher-resolution images. ${ }^{19}$ The planes were fixed at a distance of $20 \mathrm{~cm}$ for the work presented in this thesis.

The 3x3-module SCoTSS detector is equipped with a Global Navigation Satellite System (GNSS) antenna for geolocation purposes. The on-board GNSS provides second-by-second coordinates. Note that GNSS is an umbrella term that refers to any system of satellites which provides positioning information. Specific examples of GNSS satellite constellations include the Global Positioning System (GPS) network owned by the United States government and the Globalnaya Navigazionnaya Sputnikovaya Sistema (GLONASS) network owned by Russia. The positioning accuracy of GNSS depends on the arrangement of available satellites and on the environment in which the antenna is placed, but is typically $2-3 \mathrm{~m}$.

The detector is also equipped with an optical camera mounted at the front of the enclosure, in front of the scatter plane, with a field of view of approximately $\pm 53^{\circ}$ in the horizontal direction. The optical camera allows for images of the operational environment to be acquired in real time, onto which a gamma-ray image may 
be co-registered for in-situ visualization of gamma-ray sources in the environment. Formation of gamma-ray images is described in detail in chapter 4 .

\subsection{Sensitive material: $\mathrm{CsI}(\mathrm{Tl})$}

Scintillating CsI $(\mathrm{Tl})$ crystals are used in the 3x3-module SCoTSS detector because of their good energy resolution and sensitivity characteristics, as well as for their reasonably robust mechanical properties. ${ }^{19,49}$ Additionally, the spectral emission profile of $\mathrm{CsI}(\mathrm{Tl})$, which was presented in figure 2.9 when discussing the properties of $\mathrm{CsI}(\mathrm{Tl})$, is well-suited for the SiPMs used in the detector, whose photon detection efficiency was presented in figure 2.14. The range of spectral emissions from $\mathrm{CsI}(\mathrm{Tl})$ extends from approximately $350 \mathrm{~nm}$ to $700 \mathrm{~nm}$ and peaks at approximately $550 \mathrm{~nm}$. Although the peak photon detection efficiency for the SiPMs used in the detector occurs at approximately $420 \mathrm{~nm}$, the range of wavelengths to which the SiPMs are sensitive includes the full spectrum of $\mathrm{CsI}(\mathrm{Tl})$ emissions, extending from approximately $300 \mathrm{~nm}$ to $950 \mathrm{~nm}$.

All crystals are cubic in shape. Those in the absorber plane measure $2.8 \mathrm{~cm}$ per side, while those in the scatter plane measure $1.35 \mathrm{~cm}$ per side. ${ }^{19}$ With these dimensions, the $3 \times 3$-module configuration provides a total of $15.8 \mathrm{~kg}$ of sensitive material. The dimensions of the crystals in the scatter plane were chosen in order to minimize the probability of a single photon undergoing multiple scattering events, while still maintaining good position and energy resolution. Similarly, the dimensions of the absorber plane $\mathrm{CsI}(\mathrm{Tl})$ elements were chosen to give a high probability of absorbing all of the energy of the photons scattered in the scatter plane, while still maintaining reasonable spatial resolution. MC simulation studies were used to optimize the crystal dimensions and achieve the required balances mentioned above, using the procedure described in reference 31 . 


\subsection{Light readout: SiPMs}

The scintillation light produced by the CsI(Tl) crystals is measured using SiPMs produced by SensL* (model MICROFC-60035-SMT-TR1). SiPMs were chosen because of their thin profile, which introduces minimal dead material into the detector. ${ }^{49}$

The individual SiPMs are $6 \mathrm{~mm}$ in size and employ $35 \mu \mathrm{m}$ microcells, for a total of 18,980 microcells per SiPM and a fill factor of $64 \% .{ }^{38}$ Within the scatter plane each $\mathrm{CsI}(\mathrm{Tl})$ crystal is coupled to a two-by-two array of SiPMs, and within the absorber plane each $\mathrm{CsI}(\mathrm{Tl})$ crystal is coupled to a three-by-three array of SiPMs. Figure 3.2 shows examples of three SiPM arrays of the type used in the scatter plane of the 3x3-module SCoTSS detector, where a single SiPM has been outlined with a dashed red line and pointed to by a red arrow for context. The signals produced by each $\mathrm{SiPM}$ in an SiPM array are summed together when measuring scintillation light. Each $\mathrm{CsI}(\mathrm{Tl})$ crystal element in the $3 \times 3$-module SCoTSS detector is individually coupled to an SiPM array and is optically isolated.

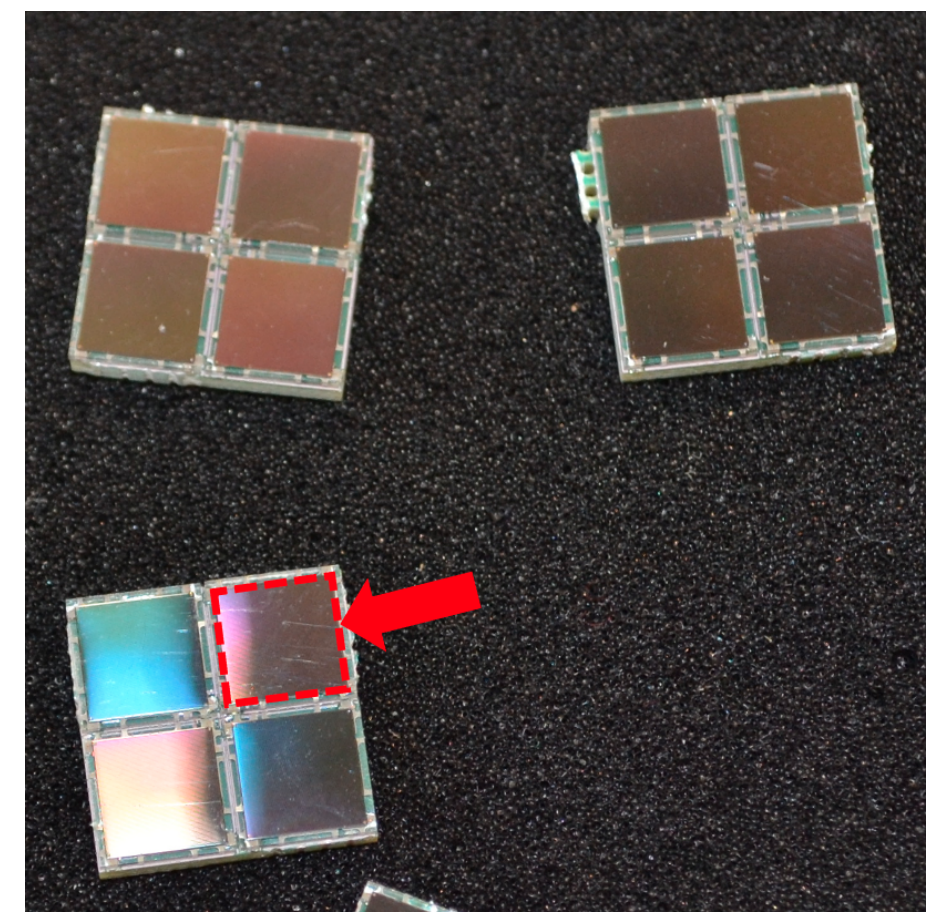

Figure 3.2: Examples of SiPM arrays used to read scintillator elements in the scatter plane of the 3x3-module SCoTSS detector. An individual SiPM in one of the arrays has been outlined with a dashed red line and indicated by the red arrow.

*SensL (Cork, Ireland) was integrated into ON Semiconductor in 2018. 
The photon detection efficiency for the model of SiPMs used in the 3x3-module SCoTSS detector was presented in figure 2.14 when discussing the operating principles of SiPMs. The spectral range of the SiPMs extends from $300 \mathrm{~nm}$ to $950 \mathrm{~nm}$ in wavelength, with peak photon detection efficiency at approximately $420 \mathrm{~nm} \cdot{ }^{38}$ As was discussed in section 3.2, this range is sufficient to cover the emission spectrum of the $\mathrm{CsI}(\mathrm{Tl})$ scintillators used in the detector.

The SiPMs are operated at a constant bias of $30 \mathrm{~V}$. The breakdown voltage increases by $21.5 \mathrm{mV} /{ }^{\circ} \mathrm{C}$ for the model of SiPM used in the $3 \times 3$-module SCoTSS detector. ${ }^{38} \mathrm{~A}$ variation in the breakdown voltage changes the overvoltage applied to the SiPM as the overall bias is held constant. The effect of changing overvoltage on the dark-count rate for the model of SiPMs used in the 3x3-module SCoTSS detector was presented in figure 2.15 when discussing dark-count rates in SiPMs. Likewise, the temperature dependence of the dark-count rate for the model of SiPM used in the 3x3module SCoTSS detector was presented in figure 2.16. Methods of correcting for the change in response of the combined $\mathrm{SiPM} / \mathrm{CsI}(\mathrm{Tl})$ detector elements to environmental factors such as temperature are presented in chapter 4.

\subsection{Detector electronics and data acquisition}

The 3x3-module SCoTSS detector is intended for use in mobile survey applications. Therefore, custom miniaturized electronics were developed to achieve portability, and the instrument can be powered from a $12 \mathrm{~V}$ battery. The custom electronics serve the 288 channels of the detector, where a channel is defined as a CsI(Tl) and SiPM unit. The electronics provide the SiPM voltage bias of $30 \mathrm{~V}$. The electronics also provide signal pre-amplification and noise filtering.

Pulses produced in each channel of the 3x3-module SCoTSS detector are sampled and digitized every $16 \mathrm{~ns}$ and at a resolution of 14 bits. Samples that rise above a channel-dependent voltage threshold, specified in analogue-to-digital converter $(\mathrm{ADC})$ counts, trigger the readout, causing the pulse to be integrated within a $4 \mu$ s window. The pulse integration is done on board the digitizers in the detector and the pulse profile is not saved.

For the purposes of illustrating a representative pulse profile, figure 3.3 shows a 
sample pulse profile from a single $\mathrm{CsI}(\mathrm{Tl}) / \mathrm{SiPM}$ unit irradiated by Cs-137, measured using a CAEN DT5724 14-bit desktop digitizer ${ }^{50}$ and digitized at a rate of $100 \mathrm{MHz}$. The vertical axis is in units of ADC values. The pulse and pedestal integration windows that were used with the CAEN DT5724 desktop digitizer are illustrated in figure 3.3 as a set of finely dashed red and coarsely dashed blue lines, respectively.

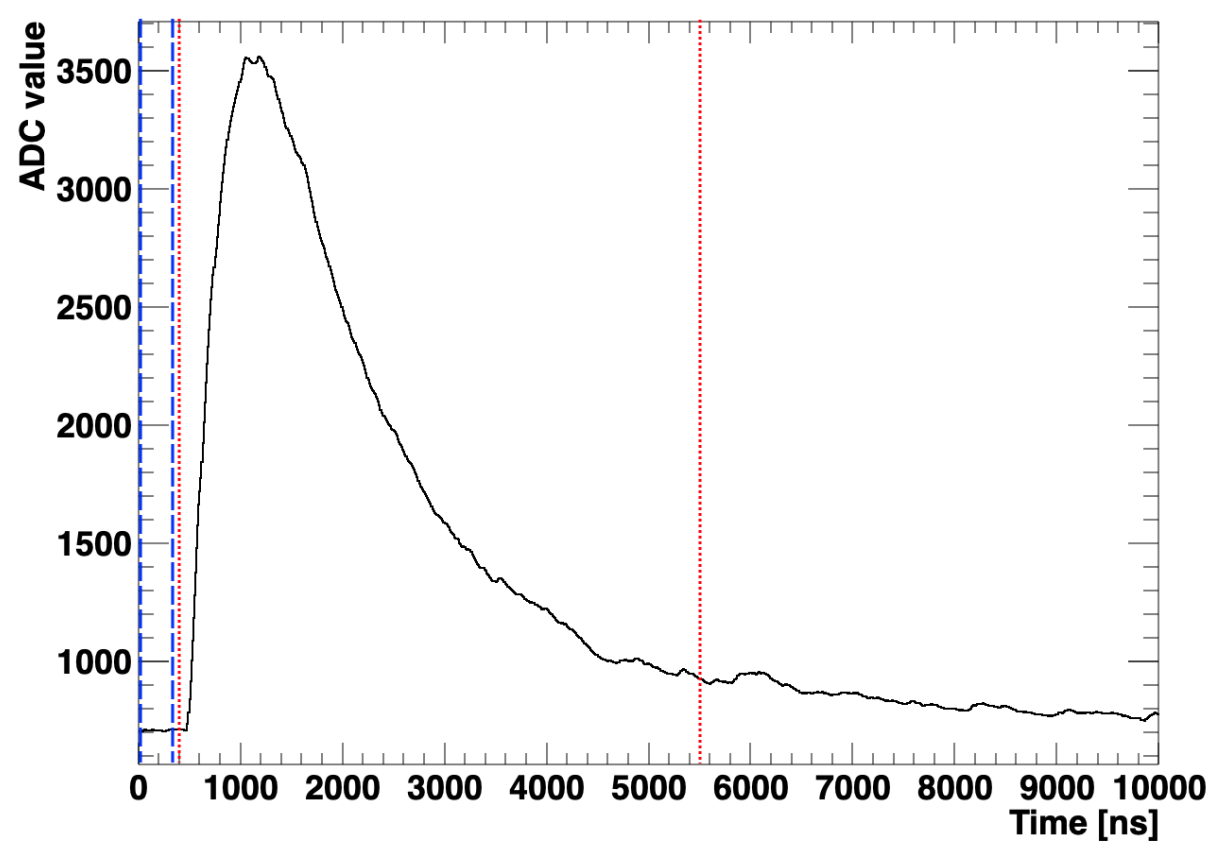

Figure 3.3: Pulse profile measured from a CsI(Tl)/SiPM unit irradiated by Cs137, measured using a CAEN DT5724 14-bit desktop digitizer and digitized at a rate of $100 \mathrm{MHz}$. Finely dashed red lines indicate the window within which the pulse was integrated, and coarsely dashed blue lines indicate the window within which a measurement of the pedestal was taken.

The pulse integration process is done simultaneously for all channels in the detector when a trigger is generated in any one channel. All channels are integrated in the time window defined by the triggering channel, taking into account the baseline level of the channel. A channel-dependent cut on the pulse areas in ADC counts is applied to the integrated pulse values to reduce noise contributions. Only those hits above a user-defined noise threshold are written to disk. The relationship between energy in $\mathrm{ADC}$ counts and in $\mathrm{MeV}$ was determined in a separate process using multiple sources with different photopeak energies. The ADC values in the event record on 
disk are converted to $\mathrm{MeV}$ using this calibration curve and are available for further processing. The event record thus contains records of hits with the number of hits per event, their channel numbers, the deposited energies, and the timestamp of the triggering channel.

The event record is examined for events that contain two hits, one in the scatter plane with at least $12 \mathrm{keV}$ of deposited energy, one in the absorber plane with at least $17 \mathrm{keV}$ of deposited energy, and with a combined total energy of at least $150 \mathrm{keV}$. This reduced sample of two-hit events is then written to disk for Compton image reconstruction. Figure 3.4 shows an example of a two-hit spectrum from an experimentally measured Cs-137 point source using the 3x3-module SCoTSS detector. A function comprised of a Gaussian peak superimposed on a linear function has been fit to the peak at $662 \mathrm{keV}$, and the achieved energy resolution $R$ of $7.5 \%$ has been indicated at the photopeak.

Figure 3.5 shows experimentally-measured energy spectra for a single CsI(Tl)/SiPM detector component from each detector plane for a Cs-137 point source, measured using a CAEN DT5724 14-bit desktop digitizer. Figure 3.5a shows a spectrum from a representative scatter plane component, while figure 3.5b shows a spectrum from a representative absorber plane component. In both subfigures, a solid red line indicates a fit of a Gaussian-plus-straight-line function that was used in calculating the energy resolution at $662 \mathrm{keV}$, which has also been indicated within each subfigure.

A separate event record is generated wherein the total energy is histogrammed on a second-by-second basis and saved with the current GNSS coordinates of the detector. The per-second energy histograms are displayed in real time, allowing for operators to monitor data quality during an exercise along with spatially-tagging locations where there are notable gamma-ray emissions. 


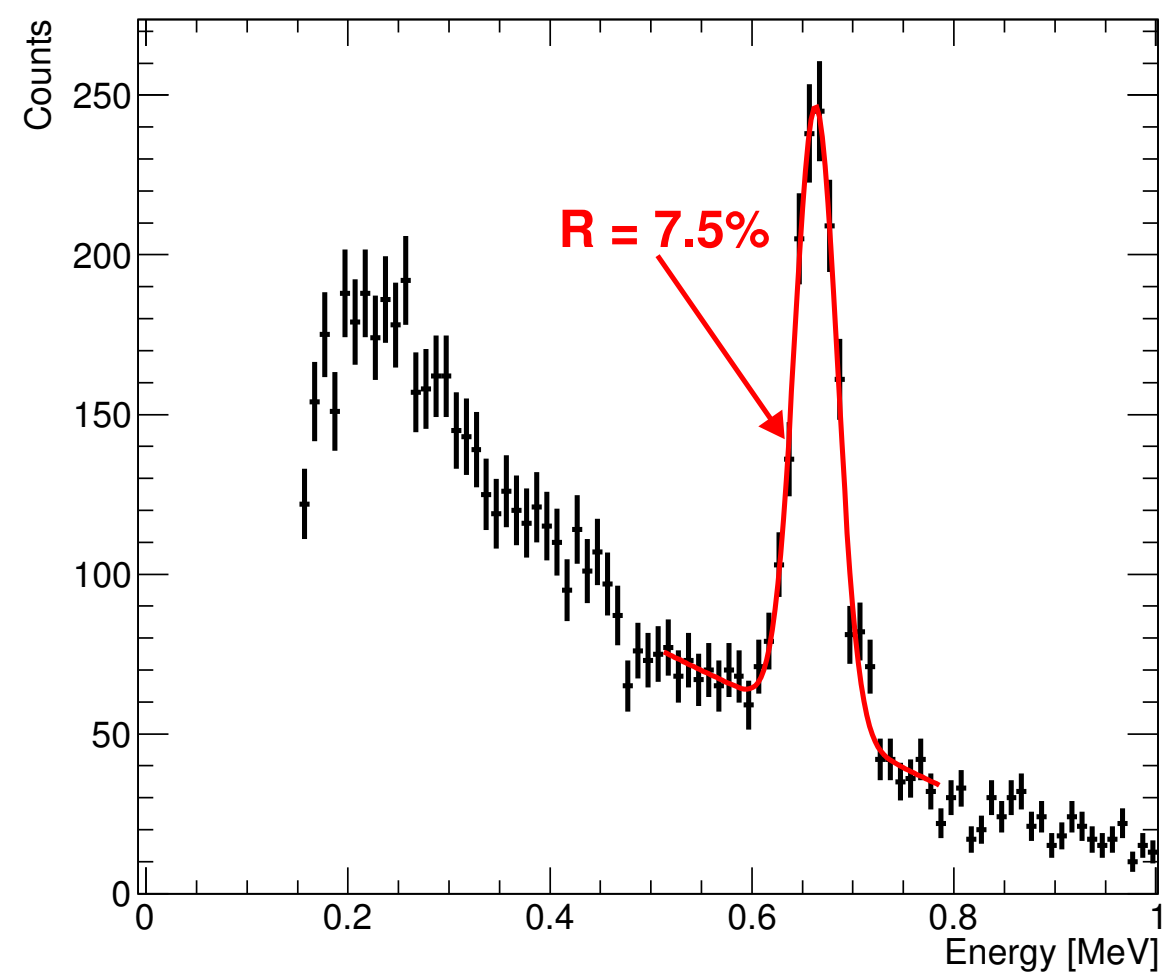

Figure 3.4: Total energy spectrum for two-hit events measured from a Cs-137 point source using the 3x3-module SCoTSS detector. The solid red line is a fit of a Gaussian peak with a linear function added to it, and the energy resolution $R$ has been indicated at the 662 keV peak. 


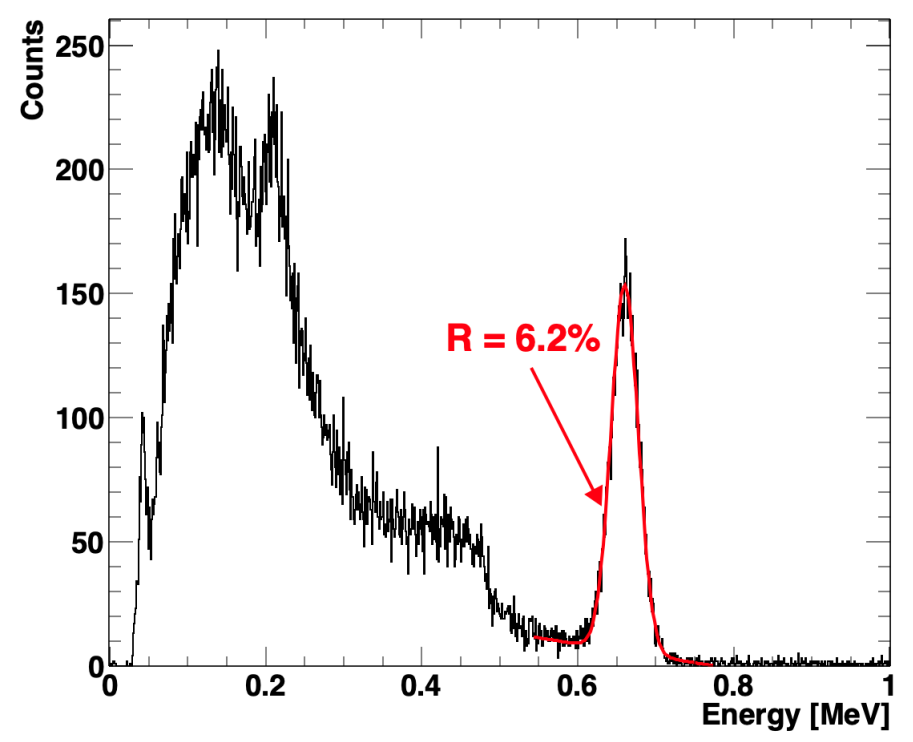

(a)

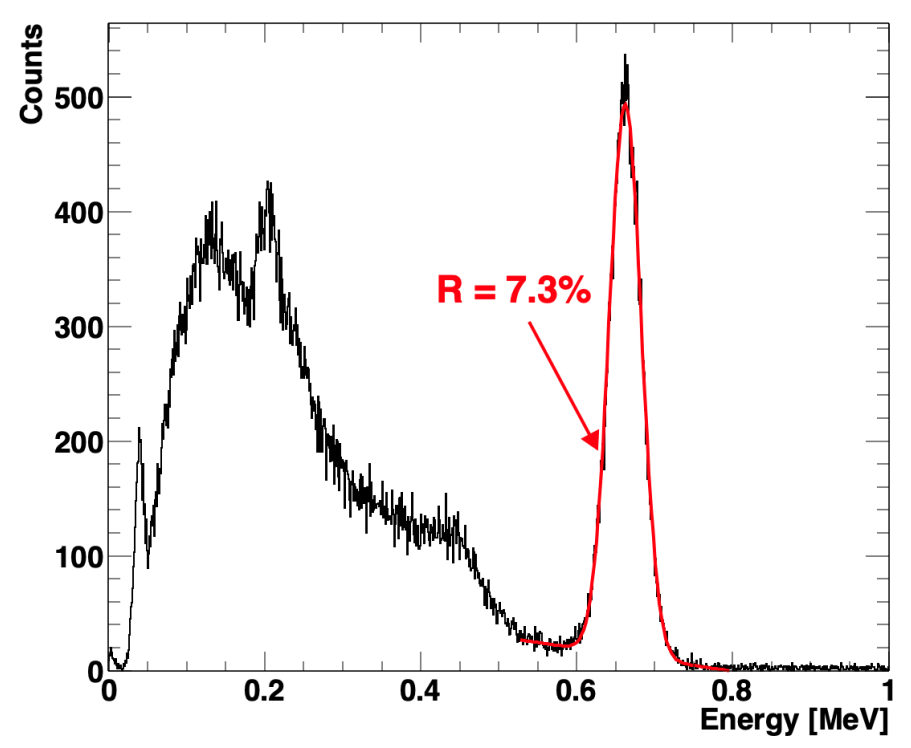

(b)

Figure 3.5: Representative Cs-137 energy spectra for single detector components for experimental data, measured using a CAEN DT5724 desktop digitizer. a) Scatter plane CsI(Tl)/SiPM channel. b) Absorber plane CsI(Tl)/SiPM channel. In both subfigures the solid red line is a fit of a Gaussian peak with a linear function added to it, and the energy resolution $R$ has been indicated at the 662 keV peak. 


\section{Chapter 4}

\section{Common methodology}

This chapter presents methodology that is common to both the Cs-137 point source experiment presented in chapter 5 and the distributed La- 140 source experiment presented in chapter 6 . The calibration procedure for the $3 \times 3$-module SCoTSS detector is presented, and the event-selection criteria used when reconstructing data acquired with the detector are outlined. The process of forming a Compton gamma image is then detailed, as is the process of creating tomographic images from the acquired data. The MC representation of the 3x3-module SCoTSS detector is outlined, and the chapter finishes by presenting the principles of two methods employed to estimate properties of radioactive distributions.

\subsection{Energy calibration}

Due to variations in the response of the CsI(Tl) scintillators and/or the SiPMs used in the 3x3-module SCoTSS detector, such as for changing temperatures as discussed in chapter 3 , the energy scale may drift. To correct for these effects an energy calibration is performed using the $662 \mathrm{keV}$ gamma-ray emissions from a Cs-137 check source. The end result of the calibration is that each of the channels in the detector receives a unique correction factor that is used to adjust the energy scale in that channel such that the known $662 \mathrm{keV}$ emission occurs at the proper location.

To perform an energy calibration, the Cs-137 check source is brought into close proximity with the detector and the detector is allowed to accumulate data. A function made of a straight line superimposed with a Gaussian curve is fit to the photopeak 
in each channel. Figure 4.1 provides an example of an energy spectrum measured in a single absorber plane channel during an energy calibration; the blue histogram is the measured energy spectrum, the solid red line is a fit of the straight line plus Gaussian function, and the dashed magenta line marks the position of $662 \mathrm{keV}$ on the energy scale. The detector is allowed to continue gathering data until the photopeak in each channel is represented with sufficient statistics to obtain a fit of the straight line plus Gaussian function. As shown in figure 4.1, the mean of the fit to the photopeak may not correspond to the true location of $662 \mathrm{keV}$ on the energy scale and a multiplicative factor is calculated to adjust the energy scale in each channel such that the photopeak is properly aligned to $662 \mathrm{keV}$. The process is carried out simultaneously for all 288 channels.

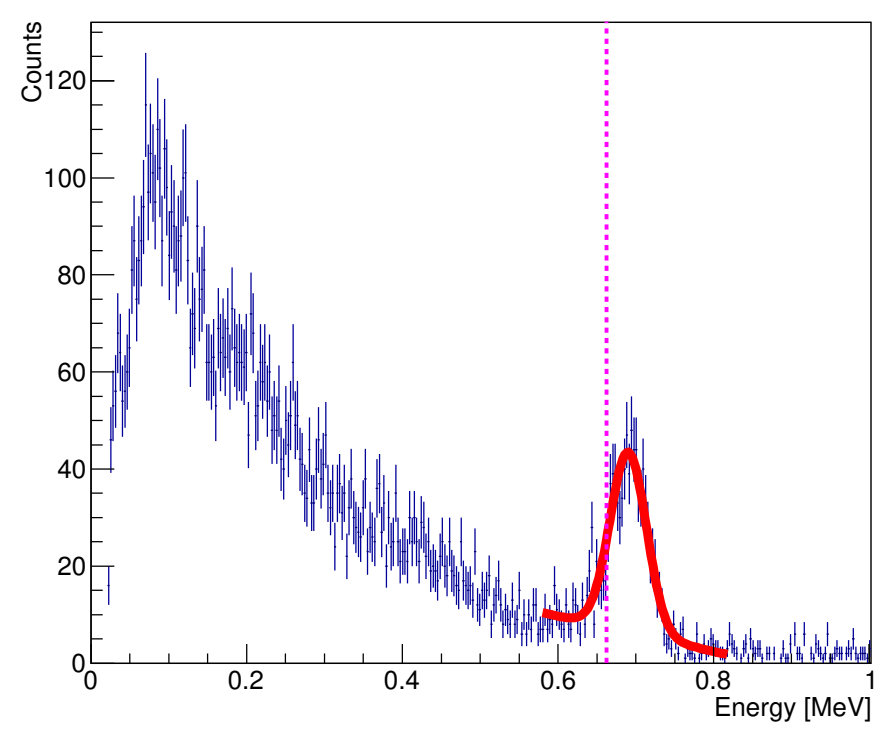

Figure 4.1: Example of an energy spectrum measured during calibration of the energy scale of the 3x3-module SCoTSS detector. The solid red curve is a fit of a straight line plus Gaussian function, and the dashed magenta line marks the position of $662 \mathrm{ke} V$ on the energy scale.

\subsection{Event selection}

This section presents the concepts behind the energy cuts that were employed in postprocessing when analyzing data acquired by the 3x3-module SCoTSS detector. The 
detector is optimized to be most sensitive in the forward direction, and the eventselection criteria presented in this section are designed to select for gamma rays that are incident first on the scatter plane. Specific numeric values will change depending on the gamma-ray emission energy that is chosen for imaging, but the motivation for the cuts follows the underlying principles given below. The event-selection criteria are presented in the order in which they are applied in analysis, and are illustrated on a representative set of experimentally measured La-140 data where the $1.596 \mathrm{MeV}$ gamma-ray emission was selected.* When analyzing simulated data, the same eventselection criteria are enforced.

The energy scale of the 3x3-module SCoTSS detector can drift slightly due to temperature-induced changes in CsI output or SiPM response when operated in outdoor experimental conditions, even after an initial energy calibration is performed. However, the drift will be small if an energy calibration has recently been performed and if the operational conditions have not changed significantly. To correct for small drifts in the energy scale in post-processing, the $1.46 \mathrm{MeV}$ emission from the K-40 contribution to the NORM background is used as a reference for a global gain drift correction. Assuming that the gain drift is constant across the energy scale, the measured energy depositions are multiplied by a common scaling factor chosen in order to align the K-40 peak in the spectrum of total energy deposited in the detector with the expected $1.46 \mathrm{MeV}$ value. This global gain correction differs from the procedure described in section 4.1 in that the correction factor is not tuned to each individual channel. Figure 4.2 demonstrates the gain correction process made in post-processing. In figure 4.2, the black histograms show a NORM background measurement, the blue histograms show a measurement of a La-140 source, and each histogram has been normalized by the acquisition time of the measurement and presented on a logarithmic scale. A dashed red line indicates the position of $1.46 \mathrm{MeV}$ on the energy scale. Figure $4.2 \mathrm{a}$ shows the measured spectra without application of a gain correction to the NORM + La-140 data; the expected K-40 peak can be seen to sit below the $1.46 \mathrm{MeV}$ mark. Figure $4.2 \mathrm{~b}$ shows the measured spectra following application of a gain correction factor of 1.03, where the K-40 peak in the NORM + La-140 data overlaps with the K-40 peak in the NORM measurement.

*The La-140 data used for illustration comes from the experiment which will be described in chapter 6 .

4.2. EVENT SELECTION 
Figure 4.3 illustrates the energy cuts used in event selection; each cut is described in further detail below. Figure 4.3a shows a two-hit total-energy spectrum, where a two-hit event is defined as an event in which one interaction occurs in each of the scatter and absorber planes. Vertical magenta lines enclose the range of energy depositions accepted by the cuts. A more detailed view of the event selection criteria is given in figure $4.3 \mathrm{~b}$, which shows the same data as a coincidence spectrum wherein the energy depositions in each detector plane measured for each two-hit event are plotted against each other. Within figure 4.3b, magenta lines enclose two regions of energy deposition combinations that are accepted by energy cuts.

A set of preselection and noise rejection cuts are enforced during post-processing after a gain correction is applied. There must be at least one interaction in each of the scatter and absorber planes, with a minimum of $15 \mathrm{keV}$ deposited in the scatter plane and $25 \mathrm{keV}$ deposited in the absorber plane. Additionally, the combined total energy of depositions in the scatter plane and the absorber plane must exceed $150 \mathrm{keV}$. Evidence of these selection criteria is visible in figure $4.3 \mathrm{~b}$, where the smaller of the two enclosed regions stops at a lower limit of $15 \mathrm{keV}$ on the scatter plane energy deposition axis.

A photopeak selection cut is then enforced that selects for events in which the total energy deposited in the detector is consistent with the gamma-ray emission of interest. For the example of the $1.596 \mathrm{MeV}$ emission from La-140, the photopeak selection cut accepts events with a total energy between $1.52 \mathrm{MeV}$ and $1.68 \mathrm{MeV}$. Within figure $4.3 \mathrm{a}$, only the photopeak selection cut is visible and is shown by the two vertical magenta lines that enclose a peak at $1.596 \mathrm{MeV}$. Within figure $4.3 \mathrm{~b}$, the photopeak selection cut defines the width of the two regions enclosed by magenta lines. This is because events with a total energy of $1.596 \mathrm{MeV}$ lie along a diagonal connecting the $1.596 \mathrm{MeV}$ points on each of the axes in figure $4.3 \mathrm{~b}$.

Each event passing the photopeak selection cut is then tested against a set of back-scatter rejection criteria. The back-scatter rejection cut is intended to reject events in which the gamma ray first interacts within the absorber plane. Events in which the gamma ray scatters first from the absorber plane and then into the scatter plane produce only a small range of energy depositions in each detector plane and the scattering angle cannot be reliably distinguished from the resulting energy 
measurements. This is evident in figure 4.4, which shows the energy deposited by a $1.596 \mathrm{MeV}$ photon as a function of scattering angle as a solid black line, and the corresponding energy of the scattered photon as a dashed red line. At high angle, the deposited and escaping energies flatten out considerably. This selection cut is thus accomplished by excluding events whose scatter plane energy deposition would be consistent with a back-scattering event, which are the events falling into the flatter regions shown in figure 4.4. For the $1.596 \mathrm{MeV}$ emission of La-140, events with a scatter plane deposition between $0.18 \mathrm{MeV}$ and $0.30 \mathrm{MeV}$ are excluded. This cut is visible in figure $4.3 \mathrm{~b}$ as the gap between the two regions enclosed by the magenta lines.

Finally, a geometrically-motivated cut is made which rejects events for which true forward-going Compton events have low probability in the 3x3-module SCoTSS detector. Events whose scatter plane energy deposition were less than $0.8 \mathrm{MeV}$ are used when imaging the $1.596 \mathrm{MeV}$ emission of La-140, and this value defines the upper limit along the vertical axis of the larger of the two enclosed regions in figure 4.3b. This cut is motivated by relative probability of a photon to scatter at shallower angles compared to larger angles, corresponding to less energy deposited in the scatter plane. The Klein-Nishina cross sections shown in figure 2.4 demonstrate the relatively large probability for photons to scatter at shallow angle with increasing energy, and it is this information that is used to guide the geometrically-motivated cut.

Table 6.3 summarizes the energy cuts presented above for reconstructing images from the $1.596 \mathrm{MeV}$ emission of La-140, and table 5.1 shows similar cuts for reconstructing images from the $662 \mathrm{keV}$ emission of Cs-137. 


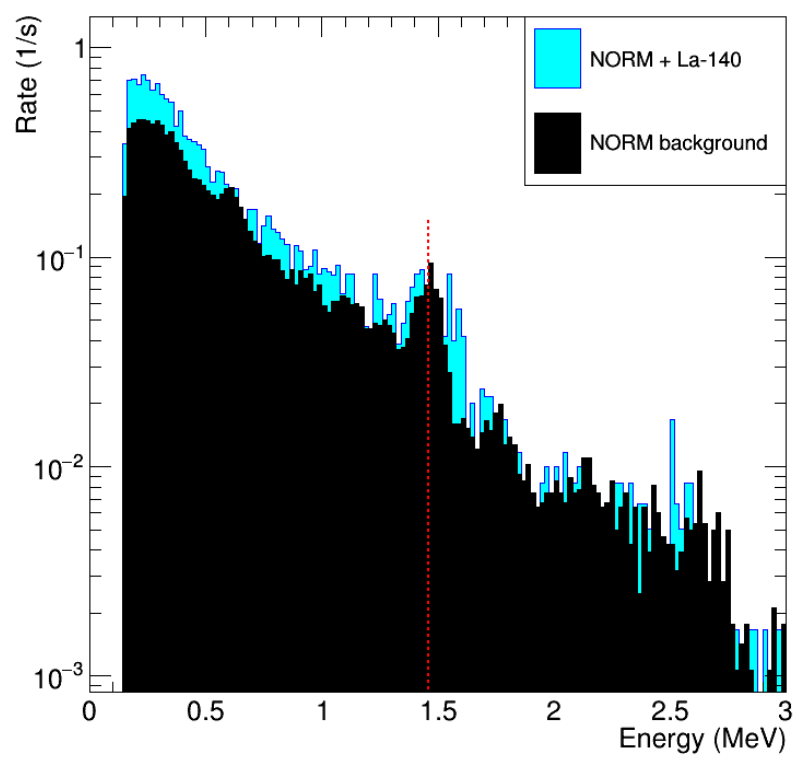

(a)

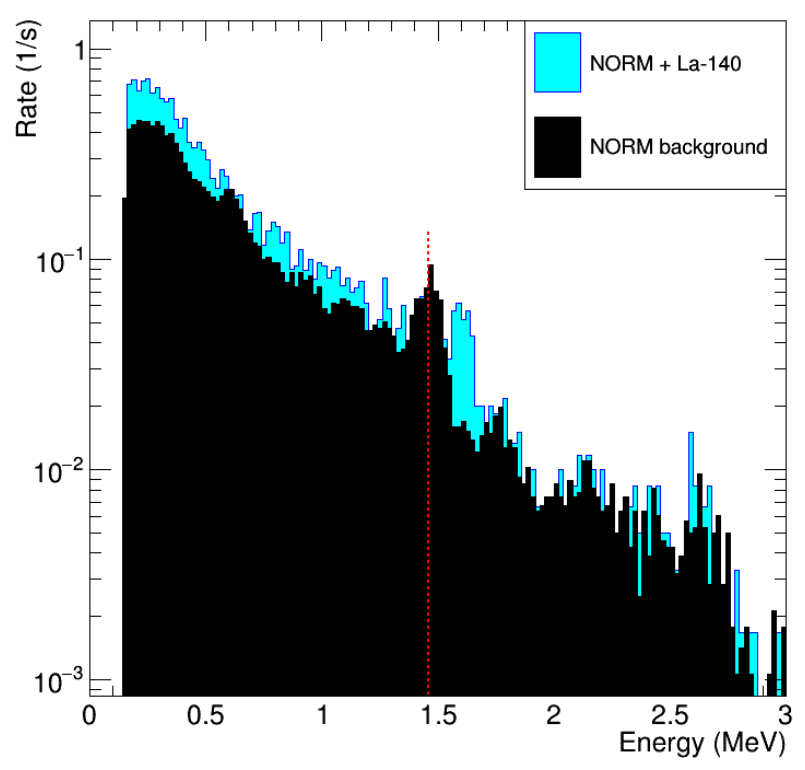

(b)

Figure 4.2: Demonstration of gain drift correction for experimentally measured La140 data. a) No gain drift correction applied. b) Gain drift correction factor of 1.03 applied. In both subfigures the histograms show the sum of energy depositions in the scatter and absorber planes, the black histogram represents a NORM background measurement, the blue histogram represents a measurement of an La-140 source, and each histogram has been normalized by the acquisition time and presented on a logarithmic scale. The dashed red line indicates the position of $1.46 \mathrm{MeV}$. Bin widths are $20 \mathrm{keV}$. 


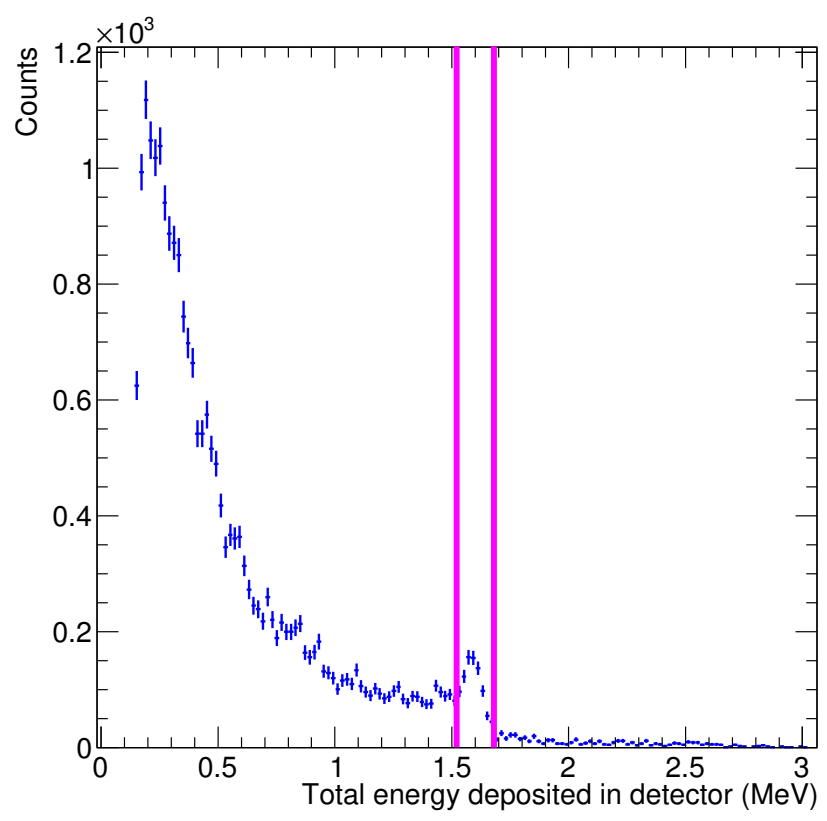

(a)

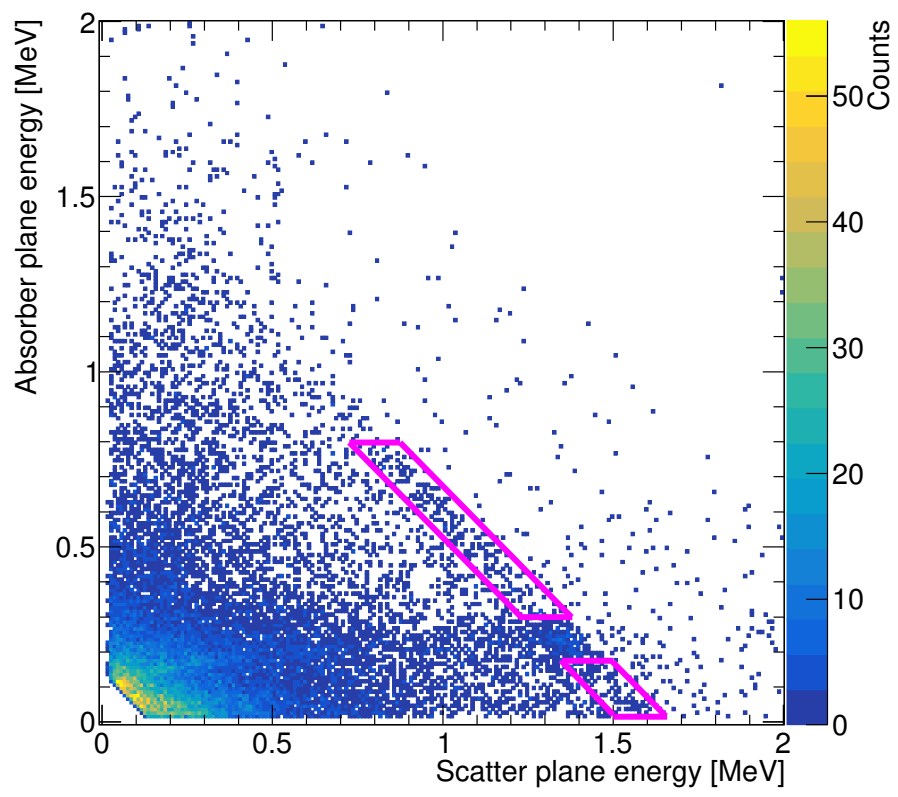

(b)

Figure 4.3: Representative illustration of energy cuts used in event selection, applied to experimentally measured La-140 data. a) Event selection criteria illustrated on a total-energy spectrum using $20 \mathrm{keV}$ bins. b) Event selection criteria illustrated on a coincidence energy spectrum using $10 \mathrm{keV}$ bins along each axis. In both subfigures, magenta lines enclose the regions accepted by the energy cuts used in event selection. See text for description of each cut. 


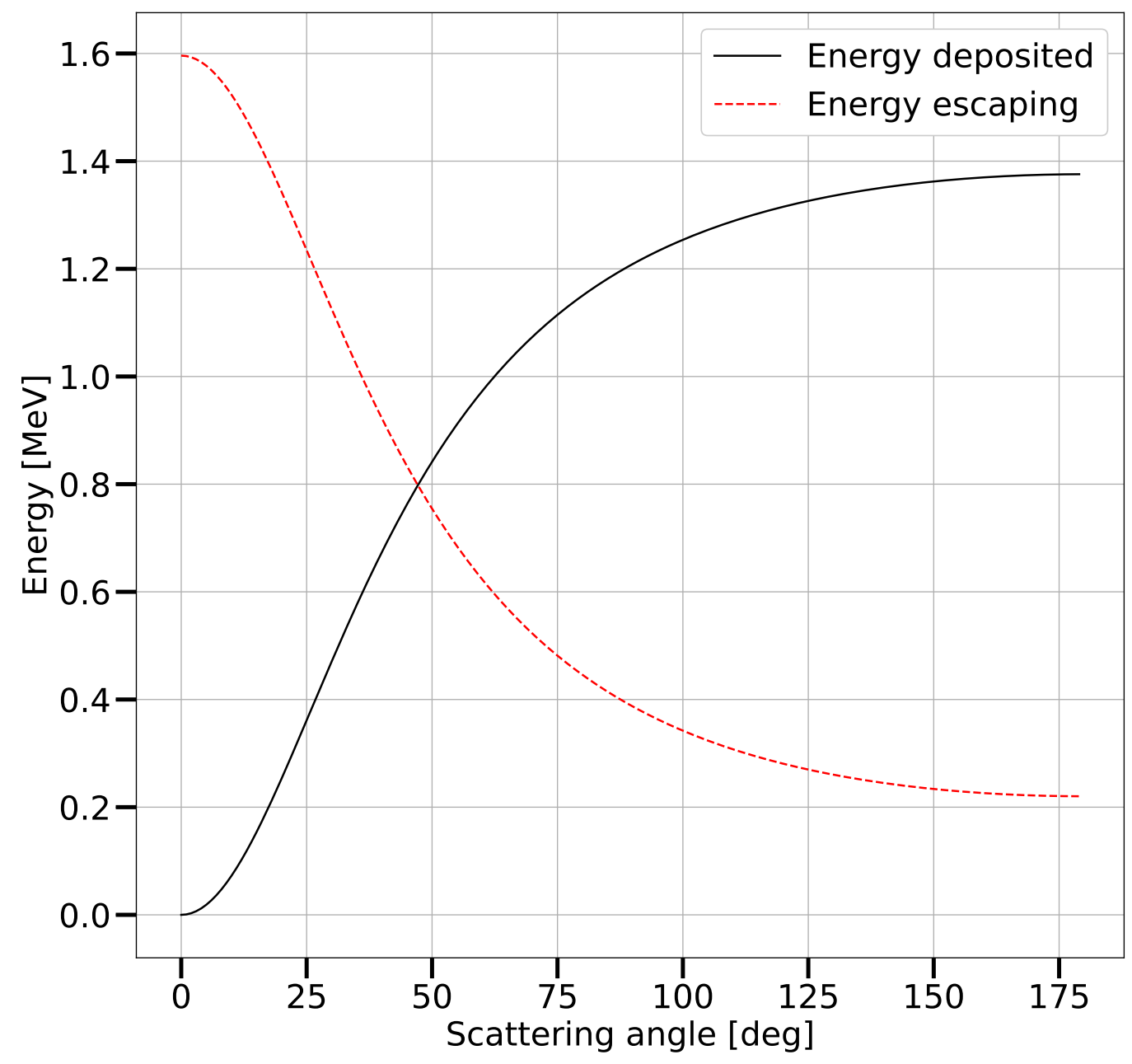

Figure 4.4: Energy exchange in a Compton scattering event as a function of scattering angle, for a $1.596 \mathrm{MeV}$ photon. 


\subsection{Background subtraction}

Background subtraction was performed by scaling the two-hit total-energy spectrum acquired from a NORM background measurement to match the shape of the two-hit total-energy spectrum resulting from a signal+background measurement. This was performed by first choosing a lower energy limit above which the signal+background spectrum contained contributions primarily from the NORM background. The value of this lower energy limit depends upon the radionuclide being measured. The sum of counts between that lower energy limit and an upper limit of $3 \mathrm{MeV}$ in each of the background and signal+background spectra was then calculated. The ratio of the calculated counts in the signal+background spectrum to that calculated in the background spectrum was taken, and the background spectrum was scaled by this ratio. A background-subtracted spectrum was then obtained by subtracting the scaled background spectrum from the signal+background spectrum. One example of a background-subtracted spectrum may be seen in figure $6.4 \mathrm{~b}$.

\subsection{Making a Compton gamma image}

Figure 4.5 shows the coordinate system used for image reconstruction with the $3 \times 3$ module SCoTSS detector. Within figure 4.5, the 3x3-module SCoTSS detector has been represented as two yellow rectangular prisms and a representative Compton cone has been illustrated as a grey surface with an axis given by a red arrow. The polar angle $\alpha$ of the cone axis is measured from the z-axis and is drawn in green in figure 4.5, while the azimuthal angle $\phi$ is measured from the $\mathrm{x}$-axis and is drawn in blue in figure 4.5. Calculation of these angles, along with the reconstruction of the Compton cone, is described below.

Consider the reconstruction of a single Compton cone. The azimuthal and polar angles, along with the complementary polar angle $\alpha^{\prime}$, are calculated as

$$
\begin{aligned}
\phi & =\arctan \left(\frac{r_{y}}{r_{x}}\right) \\
\alpha & =\arctan \left(\frac{\sqrt{r_{x}^{2}+r_{y}^{2}}}{r_{z}}\right) \\
\alpha^{\prime} & =\pi-\alpha
\end{aligned}
$$




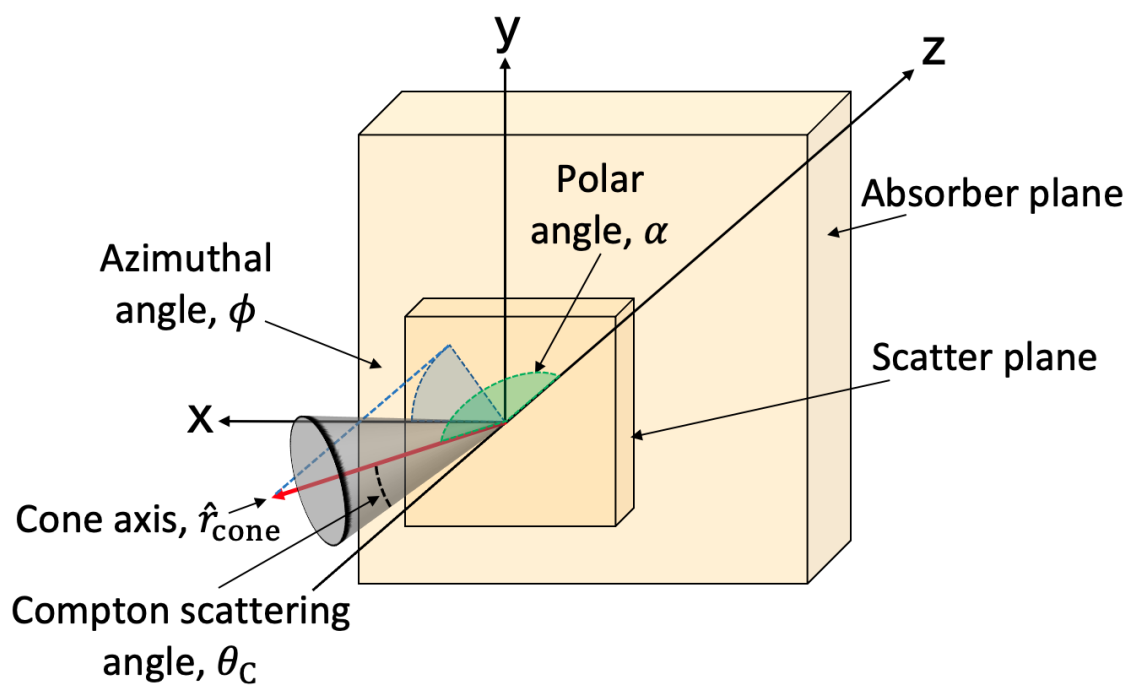

Figure 4.5: Coordinate system used for image reconstruction with the 3x3-module SCoTSS detector, which has been represented as two yellow rectangular prisms. A representative Compton cone has been shown in grey, with an axis represented by the red arrow. The azimuthal angle of the cone axis has been drawn in blue, and the polar angle has been drawn in green. Image not to scale.

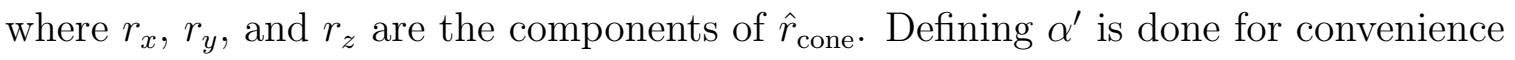
during the reconstruction process, since the source lies in the $-z$ hemisphere of the coordinate system of the $3 \times 3$-module SCoTSS detector.

The Compton scattering angle $\theta_{\mathrm{C}}$ is calculated from the energy deposits in the scatter and absorber plane, $E_{\text {sca }}$ and $E_{\text {abs }}$, respectively, via equation 2.28.

Due to the large distances involved in the measurements and image reconstructions presented in this thesis, a simplifying assumption is made that the vertex of the Compton cone is at the centre of the scatter plane rather than at the specific scatter plane crystal involved in the interaction. The Compton cone is then defined as the surface oriented with an axis along $\hat{r}_{\text {cone }}$ and at a half-opening angle of $\theta_{\mathrm{C}}$ relative to the axis, as illustrated in figure 4.5.

Figure 4.6 shows a schematic illustration of the coordinate system used to represent the Compton cone in angular coordinates, with one axis representing the horizontal angle with respect to the central axis of the 3x3-module SCoTSS detector and a second axis representing the vertical angle with respect to the central axis. The coordinate system is shown with a $\pm 53^{\circ}$ field of view along each axis for the purposes of illustration. The extent of the field of view may, however, be changed if 
needed when forming a gamma image. In this representation, the Compton cone is

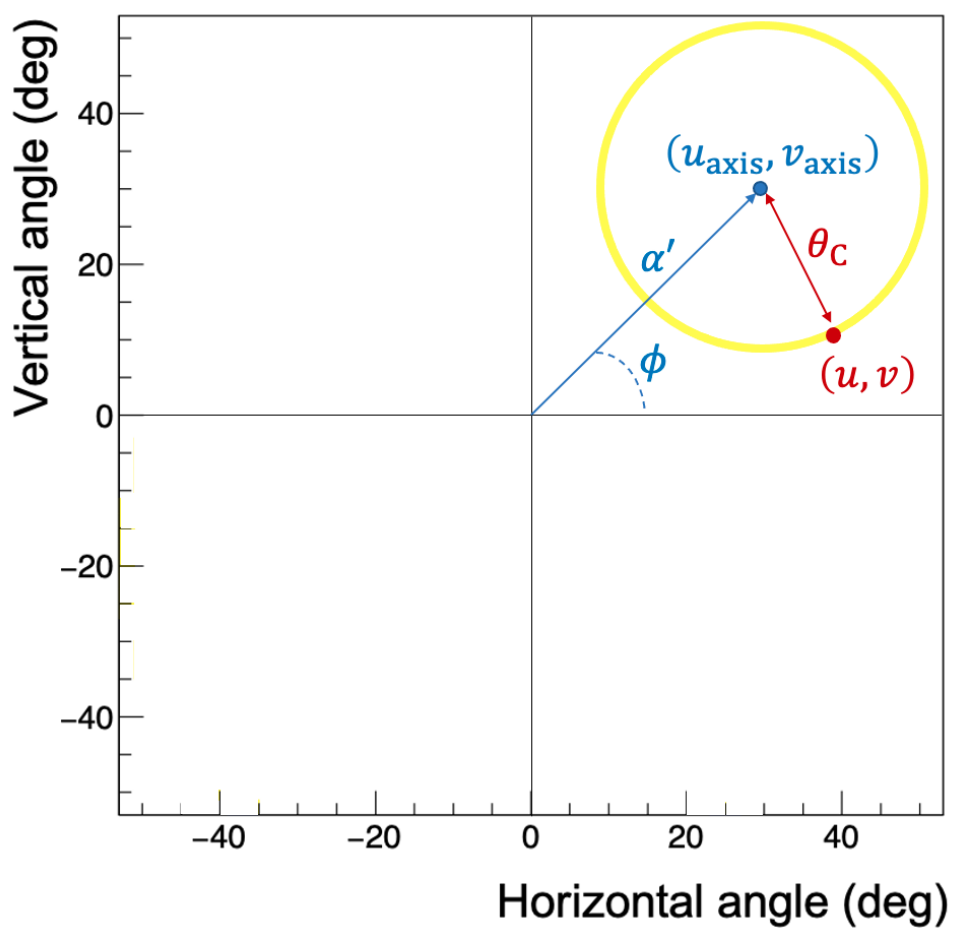

Figure 4.6: Schematic illustration of the coordinates used for plotting Compton rings in the field of view of the 3x3-module SCoTSS detector. A Compton ring is represented in yellow, with a central point $\left(u_{\text {axis }}, v_{\text {axis }}\right)$ denoted by a blue point. A single point $(u, v)$ on the Compton ring has been illustrated by a red circle.

visualized as a circular structure, known as a Compton ring, and is shown as a yellow circle. The angular coordinate system behaves as a set of polar coordinates, where the coordinates of the centre of the Compton ring $\left(u_{\text {axis }}, v_{\text {axis }}\right)$, represented as a blue point, are at a radial angular distance of $\alpha^{\prime}$ from the origin and at an angle $\phi$ from the horizontal axis. A single location $(u, v)$ on the Compton ring has been illustrated as a red point to demonstrate that points on the Compton ring form a circle with radius $\theta_{\mathrm{C}}$ about the centre of the Compton ring. The angular coordinates of the centre of the Compton ring are given by

$$
\begin{aligned}
& u_{\text {axis }}=\alpha^{\prime} \cos \phi \\
& v_{\text {axis }}=\alpha^{\prime} \sin \phi
\end{aligned}
$$

and the perimeter of the Compton ring described by

$$
\left(u-u_{\text {axis }}\right)^{2}+\left(v-v_{\text {axis }}\right)^{2}=\theta_{\mathrm{C}}^{2}
$$


Figure 4.7 shows a demonstration of building a Compton gamma image from experimentally measured La-140 data. The La-140 dataset was acquired for the experiment described in chapter 6, and is described in further detail there. A single Compton ring is shown in figure 4.7a. Events passing the selection criteria are continually reconstructed and added to the gamma image in the field of view of the 3x3-module SCoTSS detector; figure 4.7b shows the addition of several more Compton rings to the field of view. As the number of reconstructed Compton rings builds up, a fully populated Compton gamma image, shown in figure $4.7 \mathrm{c}$, is produced that indicates the position of the gamma ray source. Within figure $4.7 \mathrm{c}$, yellow indicates the highest intensity and blue indicates the lowest intensity. If in situ visualization of the gamma-ray source is desired, the Compton gamma image may be co-registered to an optical image acquired by the on-board camera. Figure $4.7 \mathrm{~d}$ shows an example of a gamma image that has been co-registered to an optical image, where the coloured contours indicate the position of the gamma-ray source.*

*The white circle shown in figure $4.7 \mathrm{~d}$ is a source-direction estimate obtained using a gammashadowing method, which is outside the scope of this thesis. Further details may be found in reference 19 . 


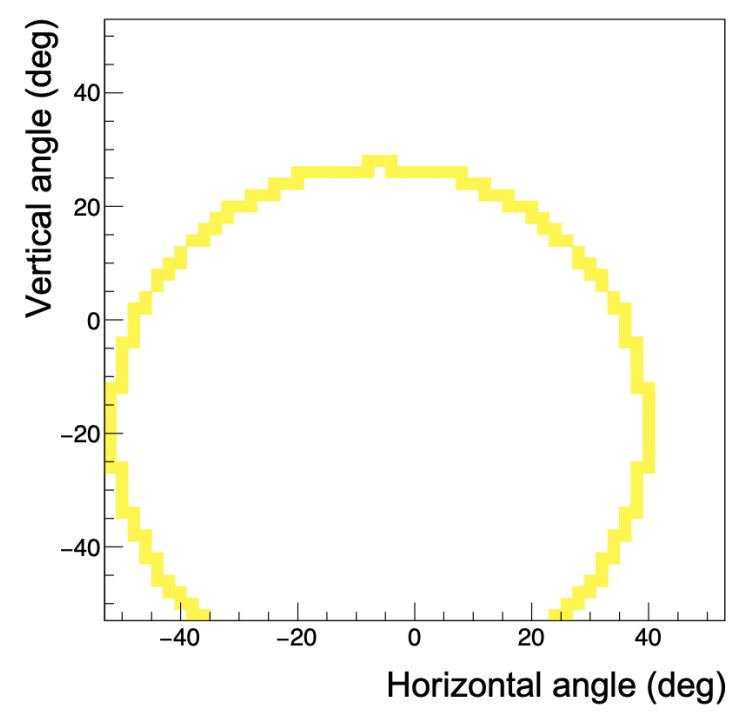

(a)

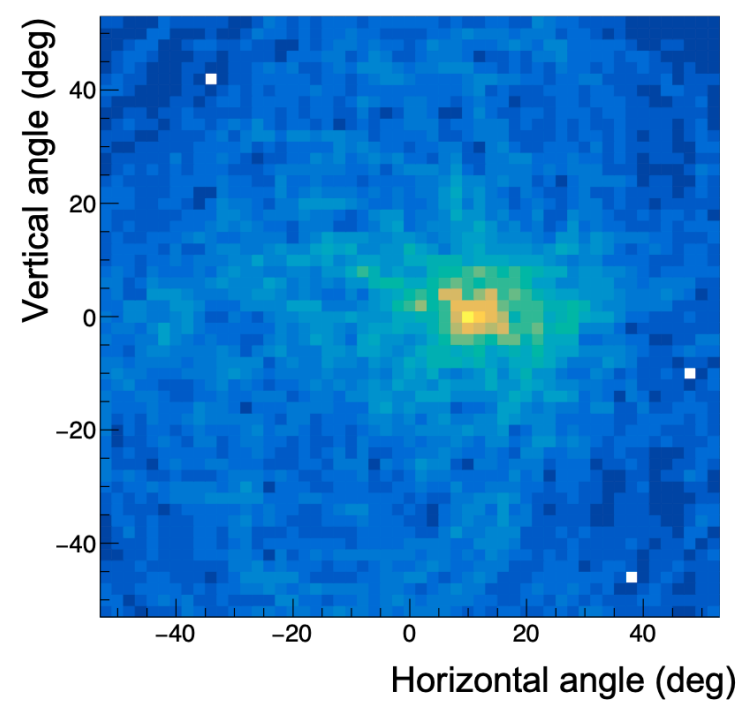

(c)

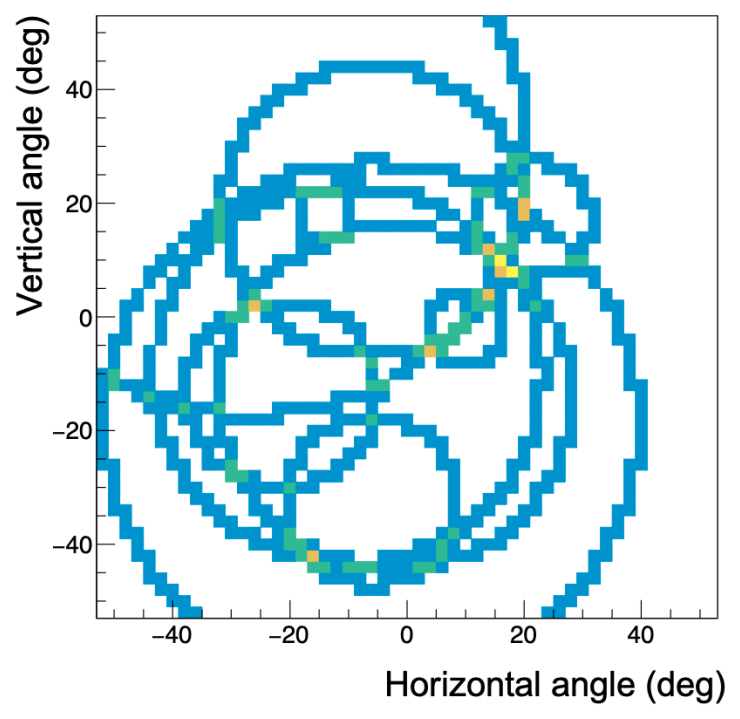

(b)

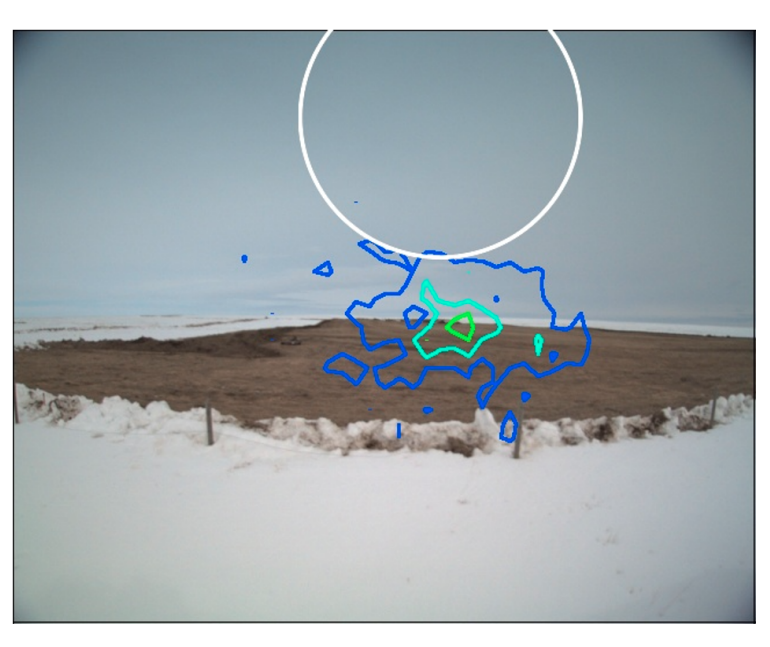

(d)

Figure 4.7: Demonstration of the Compton gamma image reconstruction process for experimentally measured La-140 data. a) A single Compton ring plotted in the field of view. b) Several Compton rings accumulating in the field of view. c) A Compton gamma image formed by a large collection of Compton rings. Blue represents the lowest intensity whereas yellow represents the highest intensity. d) Co-registration of a Compton gamma image with an optical image acquired via the on-board camera. The coloured contours indicate the position of the gamma-ray source. The white circle is a source-direction estimate obtained using a gamma-shadowing method and is outside the scope of this thesis. 


\subsection{Tomographic projection method}

The tomographic method developed and presented herein is intended for rapid reconstruction of radiation intensity maps for use in operational situations immediately following data acquisition. The method fundamentally relies on a simple superposition of back-projected Compton cones from each position at which data were acquired, referred to as dwell points. As the method is intended for expedient image reconstruction to guide operational efforts in near real time, simplifying assumptions during the reconstruction process are acceptable. An assumption is made that any gamma-ray sources lie at a constant elevation angle within the field of view of the 3x3-module SCoTSS detector, corresponding to a constant vertical angle in the coordinate system presented in figure 4.6. This simplifies the problem to that of reconstructing a twodimensional tomographic image from a collection of one-dimensional images acquired at each dwell point.

Because the work presented in this thesis involved scenarios wherein the source was known to be in front of the detector at each dwell point, the tomographic reconstruction employs a $\pm 90^{\circ}$ field of view at each dwell point.

For each event passing the selection criteria described in section 4.2 when measuring a gamma-ray source, the horizontal angles where the resulting Compton ring intersects a vertical angle of $0^{\circ}$ in the field of view are calculated and recorded. Depending on the placement of the Compton ring, it may intersect the horizon at zero, one, or two horizontal angles. For each of the recorded horizontal angles from each event, rays are back-projected to form a tomographic image. Figure 4.8 shows a schematic representation of the components involved in the back-projection of a single ray. A single Compton gamma imaging detector is represented as two grey rectangles at the right of the figure. The detector is placed next to a 10 by 10 grid; this grid represents the image space, which is a pixelated representation of the area over which the detector measured gamma radiation. The detector position around the image space in this example corresponds to the hypothetical coordinates of the dwell point at which the data were acquired. A representative pixel in the image space has been indicated by a translucent blue square. Each pixel has spatial dimensions $\Delta x$ by $\Delta y$. A back-projected ray is shown in translucent red and has an angular width of $\sigma_{\mathrm{ARM}}$. Within each pixel that the back-projected ray intersects, it can enclose an 
area $A_{\text {ray }}$ whose magnitude is in the half-bounded interval $(0, \Delta x \Delta y]$. A representative example of the area enclosed by back-projected ray is shown in solid red at the left of figure 4.8. Pixels in the image space intersected by the back-projected ray are incremented in value by a weight equal to $A_{\text {ray }} / \Delta x \Delta y$, and this back-projection process is repeated for all rays at the dwell point, superimposing the contributions from subsequent rays. This process is then repeated for each dwell point.

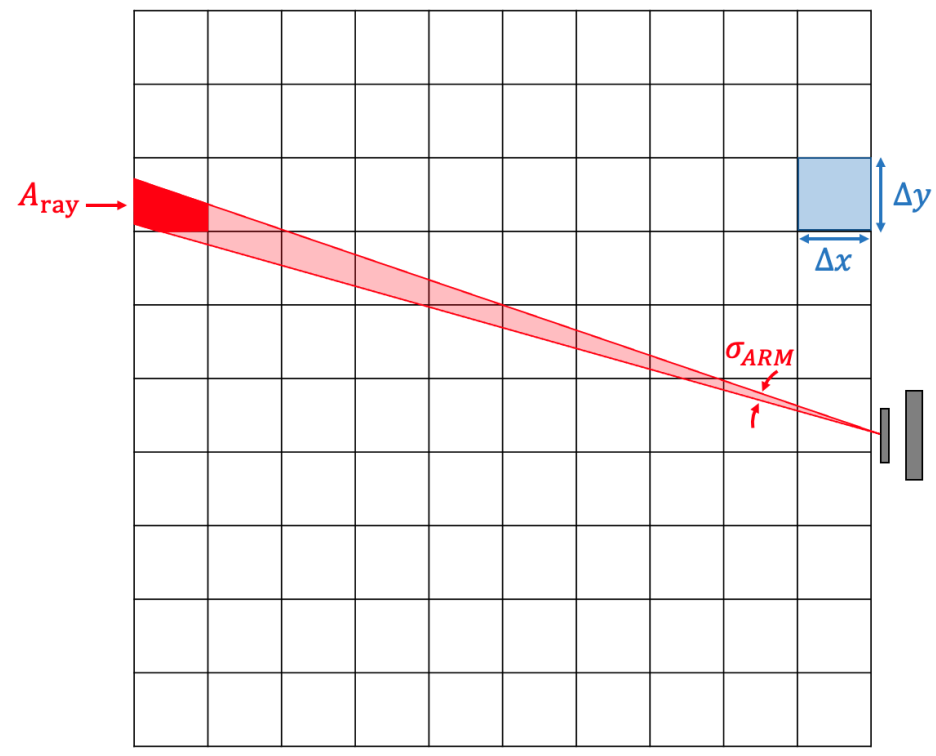

Figure 4.8: Schematic of the components of a tomographic back-projection for a single ray. A grid of black lines denotes the image space, and a representative pixel has been indicated in blue. A Compton gamma imaging detector is represented by two grey rectangles, and a single back-projected ray is represented in translucent red. An example of the area enclosed within a single pixel is represented by a solid red region.

To correct for contributions of the NORM background to the tomographic reconstructions, individual back-projection images of the NORM background from each dwell point are reconstructed following the procedure outlined above. Backgroundsubtracted back-projection images are then obtained for each dwell point by subtracting the NORM background image in a pixel-wise fashion from the signal-plus-NORM image, first scaling the NORM image by the ratio used in the background subtraction described in section 4.3. The resulting background-subtracted back-projection profiles are divided by the acquisition time of the signal measurement in order to get a temporally-normalized result. In the experiments presented in this thesis NORM measurements were acquired for the region in which the radioactive source was placed, 
but in an operationally-realistic environment this will likely be impossible. In such a scenario, one option is to acquire a NORM measurement for a nearby area and use that as a proxy for the NORM measurement at the location of the radioactive material.

A decay correction is then applied in a pixel-wise fashion to each backgroundsubtracted back-projection image at each dwell point to correct for the decay of the radioactive source material as the detector is moved between dwell points. If $N_{i}$ represents the value in the $i^{\text {th }}$ image space pixel prior to a decay correction, then the value in the same pixel following a decay correction $N_{0, i}$ is given by

$$
N_{0, i}=N_{i} \exp \left(\frac{\ln (2)}{t_{1 / 2}} \Delta t\right)
$$

where $t_{1 / 2}$ is the half-life of the radioisotope and $\Delta t$ is the time elapsed between the reference time to which the correction is being made and the timestamp at the centre of the acquisition period of the dwell point.

A dead time correction is subsequently applied individually at each dwell point, assuming a non-paralyzable detector. ${ }^{22}$ This assumption is consistent with the detector used for the work presented in this thesis. Let $M$ be the observed trigger rate in counts per second, $\tau$ be the dead time of the detector in seconds, and $N$ be the true input rate in counts per second that would be measured were $\tau=0 \mathrm{~s}$. The relationship between $N, M$, and $\tau$ is

$$
N=\frac{M}{1-M \tau} .
$$

To apply a dead time correction, the ratio $N / M$ is multiplied pixel-wise into the background-subtracted back-projection image.

Finally, to form a tomographically-reconstructed distribution of radioactivity, the corrected back-projection images from each dwell point are added together pixel-wise and the result is divided by the number of times that each pixel is contained in the field-of-view of a dwell point. 


\subsection{Monte Carlo representation of the 3x3-module SCoTSS Compton gamma imager}

Simulations were performed using the 2019 release of the EGSnrc MC code system. ${ }^{51}$ Figure 4.9 shows the interior of the simulated representation of the $3 \times 3$-module SCoTSS detector. Included in the model were the CsI $(\mathrm{Tl})$ crystals, the SiPMs, and the aluminum enclosures around each module. The SiPM arrays were modelled as a two-component geometry made from a glass $\left(\mathrm{SiO}_{2}\right)$ face and a printed circuit board (FR4 glass-reinforced epoxy laminate material). Figure 4.9a shows an exterior view of the simulated detector, where the aluminum enclosure around each module has been represented in magenta and one module in each detector plane has been highlighted for clarity. The $20 \mathrm{~cm}$ separation between the centre of the detector planes has also been indicated. Figure 4.9b shows an interior view, where the $\mathrm{CsI}(\mathrm{Tl})$ crystals have been represented as a translucent green material, the $\mathrm{SiO}_{2}$ face of the $\mathrm{SiPM}$ has been represented as a dark blue material, and the FR4 material of the printed circuit boards has been represented as a light blue material. One example of each of these components has been highlighted. Not shown in figure 4.9 is the carbon fibre material that enclosed the detector. The carbon fibre will be presented in detail in chapter 5 and chapter 6 , since the experiments described in these chapters utilized detectors with slightly different carbon fibre geometries.

The internal electronics were omitted from the simulation. Interactions with air were modelled, but ground material was not included. Omission of the internal detector electronics and of environmental ground material has a negligible effect as the event selection criteria outlined in section 4.2 preferentially select for unscattered gamma rays. Electronic noise was not simulated, since its influence on the data is suppressed by the preselection and noise cuts.

To increase the simulation efficiency, the EGS_CollimatedSource class ${ }^{52}$ was used to restrict the primary emission directions of the histories towards the simulated detector. These simulation histories will be referred to as "collimated histories", and were assigned an appropriate statistical weight to recover an isotropically-equivalent result.

To incorporate the energy resolution of the detector into the synthetic data gen- 


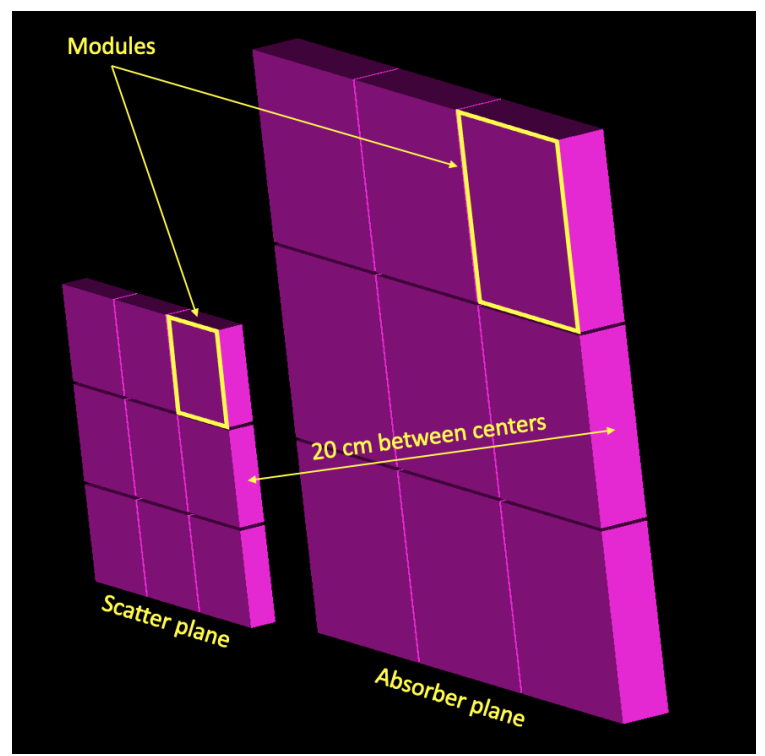

(a)

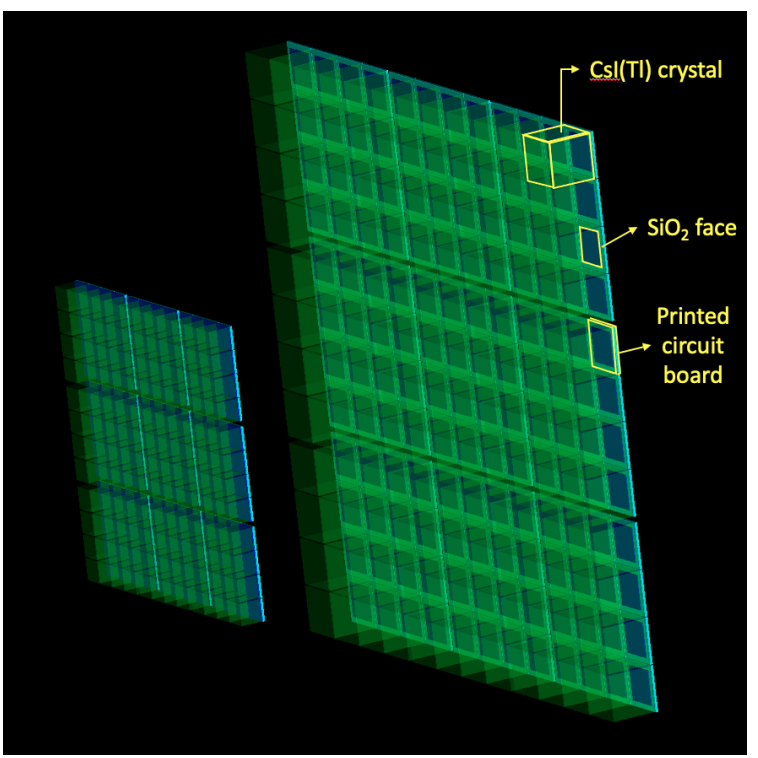

(b)

Figure 4.9: Interior view of the geometry used in MC simulations of the 3x3-module SCoTSS detector. a) Visualization with aluminum module enclosures, represented in magenta. b) Visualization with aluminum module enclosures removed. The translucent green material represents $\mathrm{CsI}(\mathrm{Tl})$, the dark blue represents the glass (SiO 2$)$ of the SiPMs, and the light blue represents FR4 material of the printed circuit boards of the SiPM arrays.

erated by $\mathrm{MC}$, the energy depositions which occurred inside the $\mathrm{CsI}(\mathrm{Tl})$ material were "smeared" with the use of an empirically-measured energy resolution function. The empirical energy resolution function used has the form

$$
R_{\% \mathrm{FWHM}}=\frac{C_{0, i}+C_{1, i} E+C_{2, i} E^{2}}{\sqrt{E}}
$$

where $R_{\% \mathrm{FWHM}}$ is the percent FWHM of a Gaussian distribution representing the photopeak that would be measured for a monoenergetic source emitting photons of energy $E$, the $C$ are fit constants, and $i$ is an index representing either the scatter plane $(i=$ sca $)$ or the absorber plane $(i=$ abs $)$. Figure 4.10 shows the values of $R_{\% \text { FWHM }}$ for each detector plane.

The value of $R_{\% \text { FWHM }}$ calculated from equation 4.9 was adjusted on a channel-bychannel basis using experimentally-measured energy resolutions at $662 \mathrm{keV}$ observed in the field to reflect the energy resolution under experimental conditions. This was achieved by scaling the right-hand side of equation 4.9 by the ratio of the observed value of FWHM at $662 \mathrm{keV}$ for a given channel to the expected value from equa- 


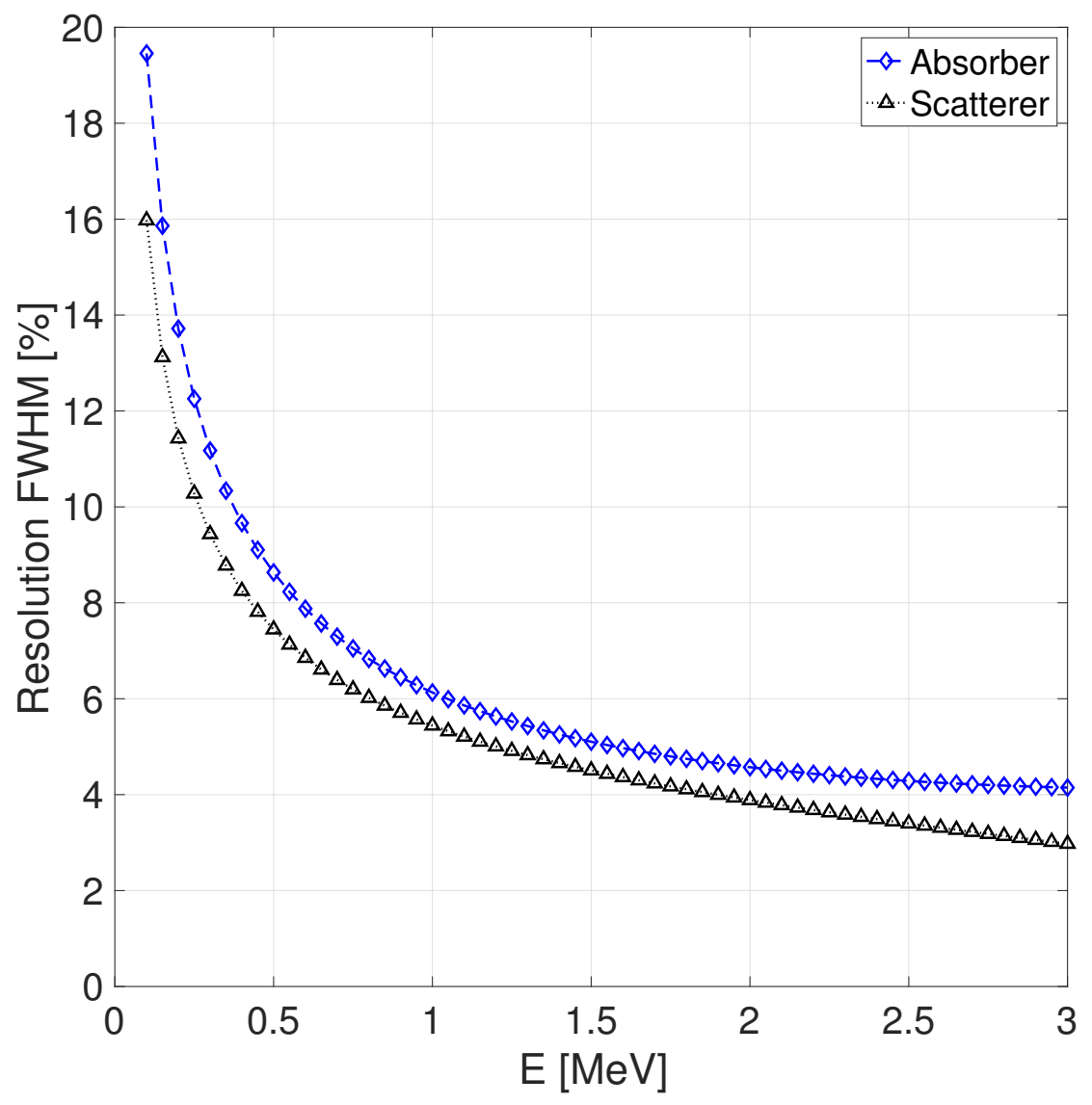

Figure 4.10: Empirical energy resolution function used to smear energy deposits in $M C$ simulation. 
tion 4.9. This method assumes that the correction applies equally across the full energy scale. The correction is calculated at $662 \mathrm{keV}$ because it is common practise to calibrate the $3 \times 3$-module SCoTSS detector with a Cs-137 check source. Assuming a Gaussian distribution, the resolution function was then converted from a percentage FWHM to a standard deviation $\sigma_{E}$ in units of energy via:

$$
\sigma_{E}=\frac{R_{\% \mathrm{FWHM}}}{2.355 \times 100} E
$$

A "smeared" energy measurement was then obtained by randomly sampling from a Gaussian distribution with mean $E$ and standard deviation $\sigma_{E}$.

The custom channel-by-channel energy smearing described above also incorporates the dead-channel statuses as they existed during the experimental data acquisition. Energy deposits in dead channels were ignored in MC simulations.

\subsection{Point-model activity estimation}

For the purposes of rapid activity estimation during an operational scenario, a method was developed wherein the unknown source distribution was modelled as a point source and the location and activity of that point source was determined using the count rates of events passing the event selection criteria at each dwell point location and the known detector efficiency from MC simulations. The efficiency must be precalculated for each gamma-ray energy to which this method is applied, but once the response of the detector has been parametrized the method allows for rapid activity estimates based on observed count rates alone (i.e. no imaging is required) that can be used in guiding operations in the field. This method was implemented for Cs-137 in chapter 5 and for La-140 in chapter 6 . This section aims to describe the general approach to the point-model activity estimate method.

The response of the detector to a point-source of a specific radioisotope was parametrized as a function of distance and angle relative to the detector as

$$
N_{\mathrm{s}}=A_{\mathrm{p}} t_{\mathrm{d}} f_{\text {dist }}(r) f_{\text {ang }}\left(\theta_{\text {axis }}\right)+\dot{N}_{\mathrm{b}} t_{\mathrm{d}}
$$

where $N_{\mathrm{s}}$ is the number of two-hit events attributed to the radioactive source of interest that pass the selection criteria outlined in section $4.2, A_{\mathrm{p}}$ is the activity of the assumed point source, $t_{\mathrm{d}}$ is the time spent measuring the source at a particular 
dwell point, $f_{\text {dist }}(r)$ is the two-hit event-detection efficiency for a point source placed at distance $r$ along the central axis of the detector and carries units of "events per decay" (i.e. the branching ratio for the emission of interest is included in the definition of $\left.f_{\text {dist }}(r)\right), f_{\text {ang }}\left(\theta_{\text {axis }}\right)$ is a unitless correction for a point source placed at angle $\theta_{\text {axis }}$ relative to the central axis of the detector (i.e. $f_{\text {ang }}\left(0^{\circ}\right)=1$ ), and $\dot{N}_{\mathrm{b}}$ is the measured rate of events contributed by the NORM background.

To obtain $f_{\text {dist }}(r)$, MC simulations were performed wherein a point source was placed at increasing distance along the central axis of the detector. The simulated data were then passed through the event-selection criteria outlined in section 4.2, using the specific energy cut values which will be presented in chapter 5 for Cs-137 and chapter 6 for La-140, and the number of events measured at each distance was then normalized by the simulated number of radioactive decays to obtain an efficiency. The resulting data were then fit with a model of the form

$$
f_{\text {dist }}(r)=C_{0} \frac{\exp \left(-C_{1} r\right)}{r^{2}}+C_{2} r+C_{3}
$$

The first term in equation 4.12 broadly includes the effects of attenuation and the inverse-square law. The physical meaning of the second and third term in equation 4.12 is not intuitive, and these terms are introduced simply to improve the ability of the model to fit the data.

$f_{\text {ang }}\left(\theta_{\text {axis }}\right)$ is determined by repeated simulations of a point source at constant distance from the detector, but at increasing angles relative to the central axis of the detector; the angular dependence of the detector response was assumed to be independent of the distance of the source from the detector. The simulated data were again passed through the event-selection criteria and normalized to the number of decays, and the ratio of all resulting efficiencies relative to the efficiency on the central axis calculated. A polynomial is then fit to the ratios using a $\chi^{2}$ minimization, where the order of the polynomial was chosen to bring the $\chi^{2}$ per degree of freedom as near to one as can be achieved. In this manner, $f_{\text {ang }}\left(\theta_{\text {axis }}\right)$ provides a unitless correction factor that accounts for the variation in the sensitivity of the detector to a point source at different angles relative to the detector.

The measured number of events is corrected for radioactive decay using equation 4.7, and the resulting number of counts used in a $\chi^{2}$ minimization fitting method in order to recover $A_{\mathrm{p}}$ along with the spatial location of the hypothesized point source. 
It should be noted that this method is of very general use and may be applied to data collected with any detector capable of measuring count rates. The use of a Compton gamma imager in addition to this quick fitting method allows for rapid in-situ visualization of the data, and the application of tomographic reconstruction techniques.

\subsection{Spatial-unfolding method}

The fundamental problem to solve is that of reconstructing an underlying distribution given a set of measurements of that distribution. A large family of iterative inversion techniques exists that could be employed for such a problem. ${ }^{53}$ Algebraic techniques, such as the Algebraic Reconstruction Technique or the Simultaneous Reconstruction Technique, treat the system response and measurements as a set of linear equations and attempt to converge on a solution by enforcing agreement with projection data. ${ }^{53,54}$ Maximum-likelihood expectation-maximization has been investigated and used in emission tomography applications, ${ }^{55-57}$ and functions by maximizing a likelihood function describing the probability of having measured the projection data that was collected given the current estimate of the image. Prior knowledge of the class of image to be reconstructed can be included in the iterative reconstruction using a Bayesian approach wherein a term representing the assumed image property (e.g. smoothness) is added to the objective function. ${ }^{53}$ For complicated measurement systems, such as in Compton gamma imaging, the full system-response matrix cannot be stored in modern computers for these algebraic or maximum-likelihood techniques, necessitating the use of list-mode approximations ${ }^{58-60}$ or other techniques.

The spatial-unfolding method developed for application to Compton gamma imaging and described in this section was inspired by a method developed for aerial radiometric survey measurement. ${ }^{48}$ Whereas the method developed in reference 48 utilizes airborne measurements with a non-directional detector that passes over the radioactive distribution, the method developed for this thesis makes use of measurements taken with a Compton gamma imager stationed at the perimeter of a zone containing the radioactive distribution.

An assumption is made that the radioactive source lies at a constant elevation

angle relative to the detector at each dwell point. The assumed elevation angles 
are presented for the specific experimental configurations in chapters 5 and 6 . Tomographic response templates are generated by applying the tomographic reconstruction methodology of section 4.5 to data obtained from MC simulation of spatially-localized tiles of activity inside of the restricted-access zone. The tomographic response templates are then added together in a weighted sum to form a test tomogram image, where the weight associated with each template indicates the underlying quantity of radioactive material ascribed to that region of space. The test tomogram is then fit to the tomogram reconstructed from measured data using a weighted least-squares minimization to extract the weights, thereby recovering an estimate of the distribution of radioactivity in the restricted-access zone. The formulation for this problem is presented in detail below.

Figure 4.11 illustrates the principles of the spatial-unfolding technique. There exists some true (but unknown) underlying distribution of radioactivity $f(x, y)$ inside of the image space, represented in figure 4.11 as a magenta shape inside of a black rhombus at the bottom-left of the figure. A Compton gamma imager measures $f(x, y)$ from several dwell points distributed around the image space, and the measurements are tomographically reconstructed to form the image $g^{\mathrm{MEAS}}$ with $N^{\mathrm{MEAS}}$ pixels; this is represented at the top-left of figure 4.11, where four grey triangles represent an arbitrary set of dwell points and $g^{\mathrm{MEAS}}$ is shown inside the image space with an arbitrary colour scale whose intensity ranges from blue (low intensity) to yellow (high intensity). A system operator $\mathcal{S}$ relates $g^{\mathrm{MEAS}}$ to $f(x, y)$ by

$$
g^{\mathrm{MEAS}}=\mathcal{S}[f(x, y)]
$$

The combined effects of geometric efficiency, attenuation due to intervening materials in the environment, the detection efficiency, the imperfect detector resolution, and the tomographic reconstruction process are all contained within $\mathcal{S}$ and result in smearing of $g^{\mathrm{MEAS}}$ relative to $f(x, y)$. A proper understanding of $\mathcal{S}$ can be used to "unfold" each point in the restricted-access zone. To determine the effects of $\mathcal{S}$, the restricted-access zone is modelled as a set of spatially-localized tiles of radioactivity $f_{i}$, where $i$ ranges from 1 to the number of fit parameters, $N^{\mathrm{PAR}}$; two examples of $f_{i}$ are illustrated in the bottom-middle and bottom-right of figure 4.11, where the image space has been divided into $N^{\mathrm{PAR}}$ tiles and the tile containing the radioactivity corresponding to $f_{i}$ is shown in orange. The minimum size of each $f_{i}$ is dictated by the angular precision of the measurement and the spatial resolution desired in the final unfolded image. 


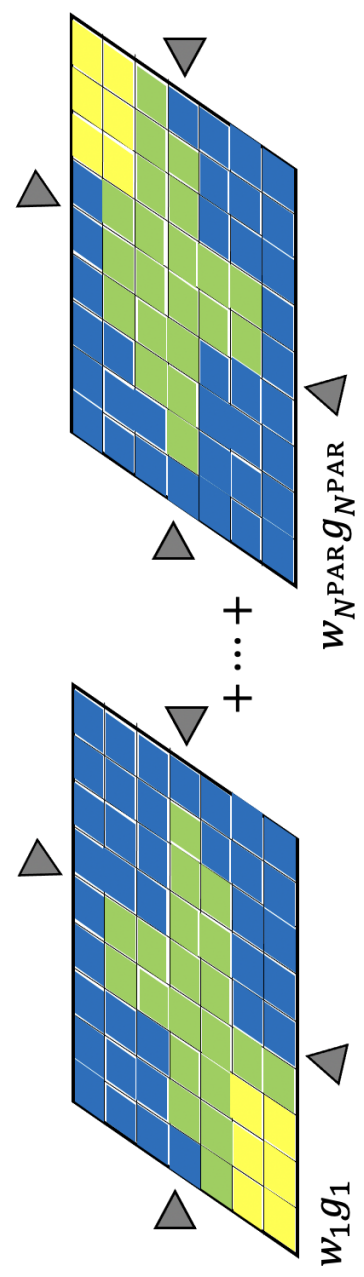

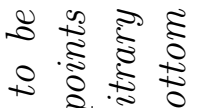

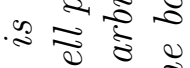

뉼

₹. ₹

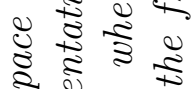

की के 3

कृ

(1) है हो

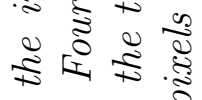

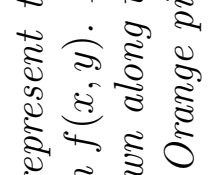

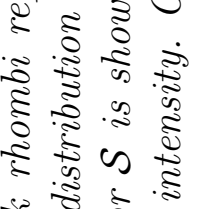

$\rightarrow$ के हो

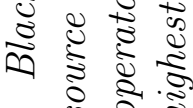

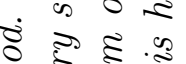

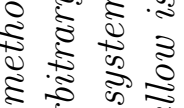

के ईू

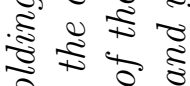

离苛

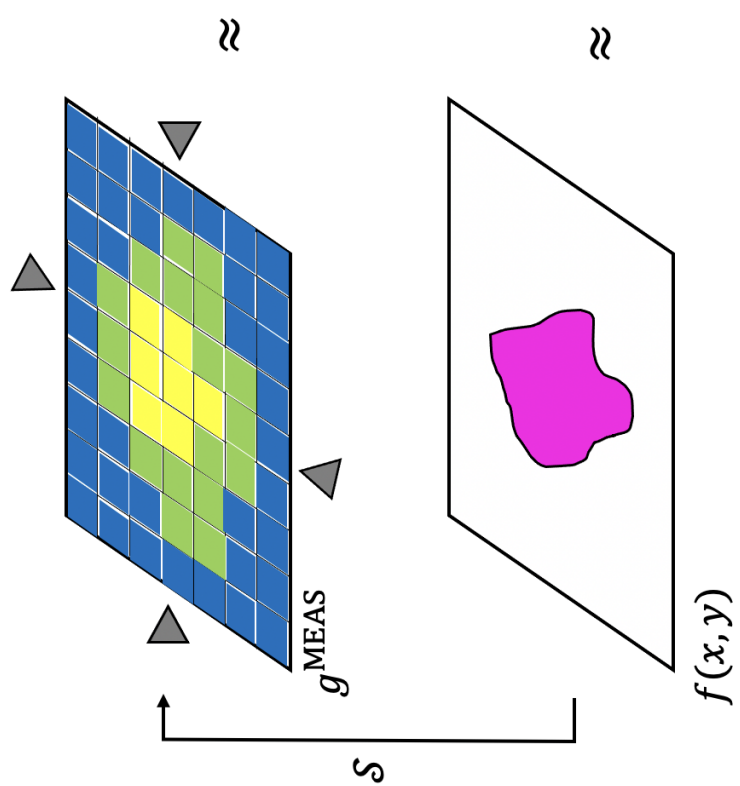

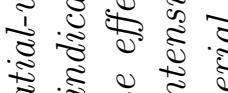

吾. 胥 का

0 ह है ₹

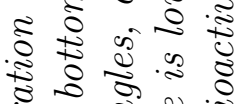

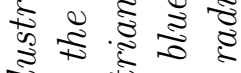

$\approx \overrightarrow{0} \approx \overrightarrow{0}$

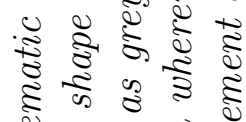

离

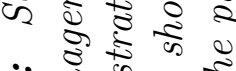

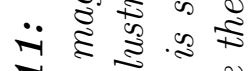

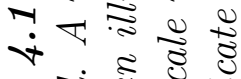

๑ ปี

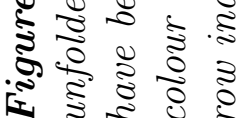


The action of $\mathcal{S}$ on $f_{i}$ is to form a tomographic response template $g_{i}$ :

$$
g_{i}=\mathcal{S}\left[f_{i}\right] .
$$

The $g_{i}$ corresponding to the $f_{i}$ shown in figure 4.11 are illustrated at the top-middle and top-right of the figure.

Calculation of equation 4.14 is performed using MC simulations to model the gamma-ray propagation and measurement process. Within the MC code, the simulated representation of the detector at each dwell point is placed relative to the $f_{i}$ with a position and orientation that matches the experimental conditions. The gamma-ray emissions from the $f_{i}$ are then propagated in space to the production of an energy deposit in the detector which can be expressed in the form of synthetic measurement data. The synthetic data thus acquired for each $f_{i}$ are then passed through the tomographic reconstruction process outlined in section 4.5 to obtain $g_{i}$, effecting the calculation of equation 4.14. A test tomogram $g^{\mathrm{TEST}}$ is then defined as a weighted sum of the $g_{i}$ :

$$
g^{\mathrm{TEST}}=\sum_{i=1}^{N^{\mathrm{PAR}}} w_{i} g_{i}
$$

where $w_{i}$ indicates the activity associated with the response template $g_{i}$. Two weights have been shown in figure 4.11 next to their corresponding templates $f_{i}$ and $g_{i} . g^{\text {TEST }}$ is then compared to $g^{\text {MEAS }}$ pixel-by-pixel, and the difference between the two images is minimized using a weighted least-squares technique in order to recover an estimate $\left\{\tilde{w}_{i}\right\}$ for the set of weights:

$$
\left\{\tilde{w}_{i}\right\}=\arg \min \sum_{j=1}^{N_{\text {MEAS }}}\left(\frac{g_{j}^{\mathrm{MEAS}}-g_{j}^{\mathrm{TEST}}}{\sigma_{j}^{\mathrm{MEAS}}}\right)^{2}
$$

where the notation $g_{j}^{\text {MEAS }}$ indicates the $j^{\text {th }}$ pixel of $g_{j}^{\text {MEAS }}, \sigma_{j}^{\text {MEAS }}$ is an estimate of the uncertainty on $g_{j}^{\mathrm{MEAS}}$, and the dependence of expression 4.16 on $\left\{w_{i}\right\}$ arises from equation 4.15. An estimator $\tilde{f}$ for $f(x, y)$ is then given by:

$$
\tilde{f}=\sum_{j=1}^{N^{\mathrm{PAR}}} \tilde{w}_{i} f_{i} .
$$

The system in expression 4.16 represents a square system, where $N^{\mathrm{PAR}}=N^{\mathrm{MEAS}}$ and there are equal numbers of parameters $w_{i}$ and pixels in $g^{\mathrm{MEAS}}$. In order to reduce 
the number of parameters, assumptions are made that some pixels have no activity associated with them and the corresponding $w_{i}$ are fixed to zero. These assumptions are discussed in more detail within chapters 5 and 6 . Additionally, expression 4.16 can easily accommodate the addition of priors, and a prior is enforced wherein all values of $w_{i}$ must be non-negative.

The Minuit2 minimization package ${ }^{61}$ which is a $\mathrm{C}++$ translation of the original Fortran-based MINUIT minimization package, ${ }^{62,63}$ was used to minimize expression 4.16 with respect to the set $\left\{w_{i}\right\}$. In particular, the Minuit2 interface provided by version 6.23.01 of the CERN ROOT Data Analysis Framework ${ }^{64} \mathrm{C}++$ toolkit was utilized. The minimization algorithm employed by Minuit2 belongs to the family of variable metric methods, and full details may be found in reference 65 . In brief, an estimate of the derivatives of the function being minimized as well as the covariance between variables are calculated at the current point in each iteration. The gradient and covariance information is used to calculate a "Newton's step" along which the minimization proceeds. The gradient and covariance estimates are updated at each new position during the minimization, and the minimization proceeds until convergence is achieved.

Estimates of the statistical uncertainty on the unfolded result were obtained using two different methods, which are referred to as the "repeated MC simulation method" and the "bootstrapping method". These methods are described in detail in the paragraphs that follow. The aim of each method is the same: to estimate the variance that may have been observed had the real experiment been repeated several times. The two methods are utilized to estimate the statistical uncertainty on the result because they have different strengths and weaknesses.

In the repeated MC simulation method, the best estimate $\tilde{f}$ of the underlying distribution of radioactive materials is built as a source geometry in a MC simulation. Repeated MC simulations are then run with unique random number generator seeds wherein the number of histories generated from the simulated representation of $\tilde{f}$ is chosen in order to produce a number of events that are commensurate with the number of events measured in the experimental data, and the simulated acquisition time is chosen such that the total activity of $\tilde{f}$ is preserved. The strength of the repeated MC simulation method is its ability to accurately incorporate all of the physics of 
particle transport through the environment and the subsequent interactions within the detector, allowing for high-fidelity simulation of the process of re-measuring the distribution of radioactive materials. However, the method is limited by the accuracy of the simulated detector and environment, as well as the agreement between $\tilde{f}$ and the true distribution $f(x, y)$.

In the bootstrapping method, it is assumed that the experimental two-hit event data acquired by the detector provides the best representation of the relative distribution of two-hit events that would be followed if the experiment were repeated. In the description that follows, the two-hit dataset has not yet been subjected to the event-selection criteria outlined in section 4.2. For a dataset with $N$ events in it, a new dataset with $N$ events is generated by resampling the original dataset with replacement, assigning to each of the original events a probability $1 / N$ of being included in the new resampled dataset. ${ }^{66,67}$ The resampled dataset is referred to as a bootstrapped dataset. Resampling of Compton imaging data requires that all information associated with each event be preserved in the resampling process. Interaction energies and Compton cone parameters are not shuffled between events, but rather the full list of information associated with a single event is placed in the bootstrapped dataset. Figure 4.12 illustrates the principles of bootstrap resampling as applied to two-hit Compton data, shown for a dataset with five events to keep the illustration simple. The original dataset is shown in black at the left of the diagram, where each row corresponds to a two-hit event and contains information regarding the energy deposits, the Compton cone parameters, and so on. At the right of the image, in blue, are three examples of bootstrapped datasets. The bootstrapped datasets were obtained by random resampling of the original dataset with replacement. Note that within the bootstrapping approach it is possible (and almost certainly true) that some of the original data will be duplicated in a bootstrapped dataset and some will be entirely omitted. The probabilities with which this occurs are accounted for by the relative representation of each sample in the original dataset. Bootstrapping offers the advantage of making no assumption regarding the distribution of the radioactive materials, and is not limited by the accuracy of a simulated model. Bootstrapping techniques have been investigated in SPECT and PET gamma imaging applications, ${ }^{68-71}$ where the method found success in predicting statistical properties of the reconstructed images such as the variance on the reconstruction. However, bootstrapping relies on 


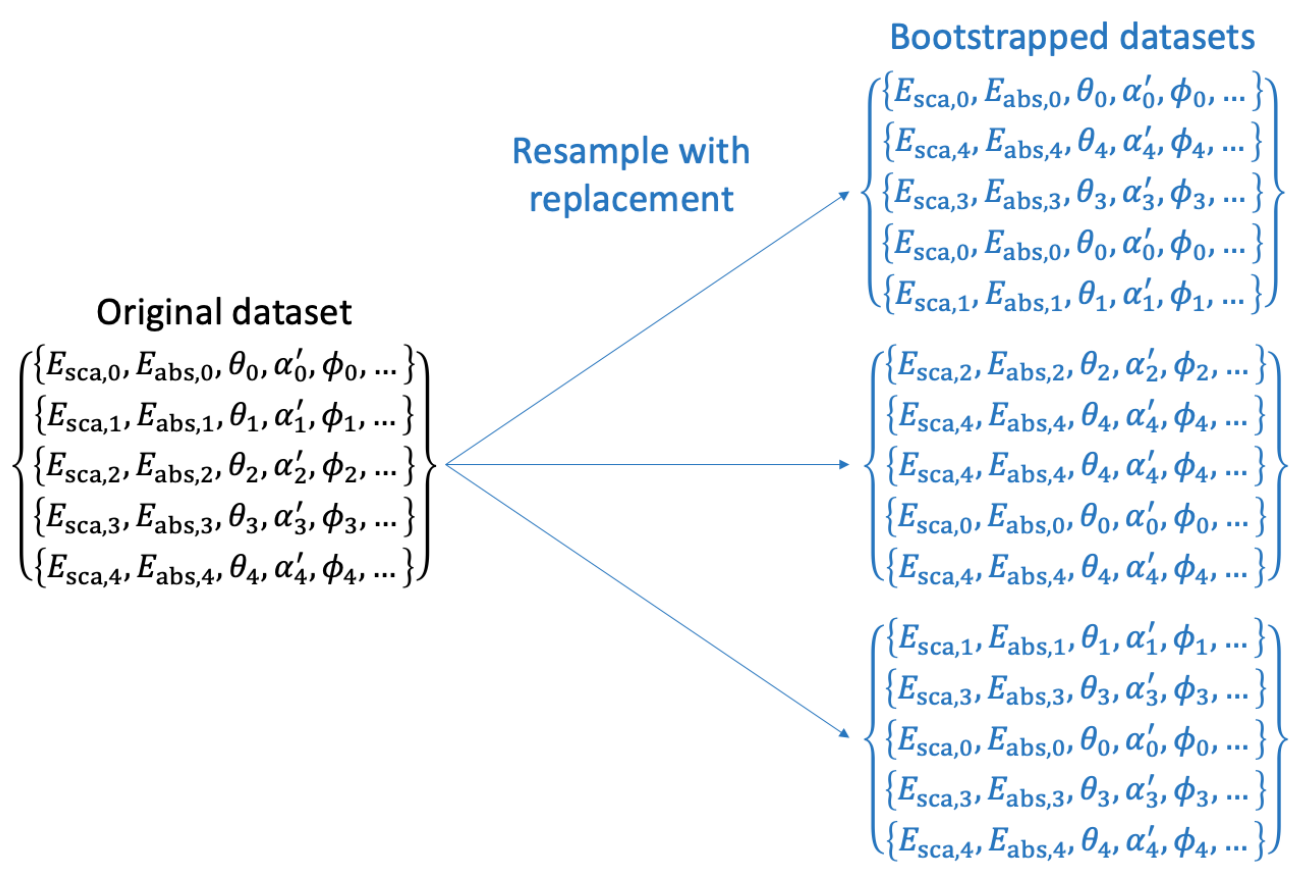

Figure 4.12: Example of bootstrap resampling of Compton event data, shown for a dataset with five events for the purposes of illustration. Each row represents a unique two-hit event.

the fundamental assumption that the original dataset contains an accurate depiction of the distribution which would be followed under repeated experimental trials. In particular, there is a risk of underestimating the uncertainties if the original dataset does not properly capture the variance of the true distribution. ${ }^{71,72}$

Because of the complementary strengths and weaknesses of the repeated MC simulation method and the bootstrapping method, both estimates of the statistical uncertainty will be presented. With both methods, the datasets produced are passed through the event selection and image reconstruction chain of analysis, and the standard deviation of the resultant images is taken pixel-wise as an estimate of the statistical uncertainty. 


\section{Chapter 5}

\section{Point-source trial}

This chapter presents a controlled laboratory experiment in which a calibrated Cs137 source was measured in an indoor NRC laboratory. The results of the analysis of this controlled dataset support the use of the proposed methodology on the more complicated dataset of chapter 6 . The proposed point-model activity-estimation technique and the spatial-unfolding methodology developed in this thesis were applied to reconstruct the source activity and position. Specifics relating to this experiment are first presented, and results are then provided and discussed.

\subsection{Methodology}

\subsubsection{Experimental setup and data collection}

The experiment took place indoors in a controlled NRC laboratory on April 9th, 2020. A calibrated source consisting of Cs-137 encapsulated in a metal cylinder and with a known surface emission rate of (17.4 \pm 0.7$)$ million $662 \mathrm{keV}$ photons per second was measured. The dimensions of the cylinder were small enough such that the source was effectively a point source, and it was treated as a point-source of $662 \mathrm{keV}$ photons in the analysis presented in this chapter.

The 3x3-module SCoTSS detector was transported to nine locations, referred to as dwell points, around the point source to acquire data. Figure 5.1 provides a schematic overview of the experimental geometry. A black cross marks the location of the point source, which had coordinates $(7.8,9.3) \mathrm{m}$ with an uncertainty of $0.5 \mathrm{~cm}$ 
on each coordinate. Each dwell point has been represented in figure 5.1 by a red diamond, with an accompanying red arrow that indicates the measured orientation of the detector.

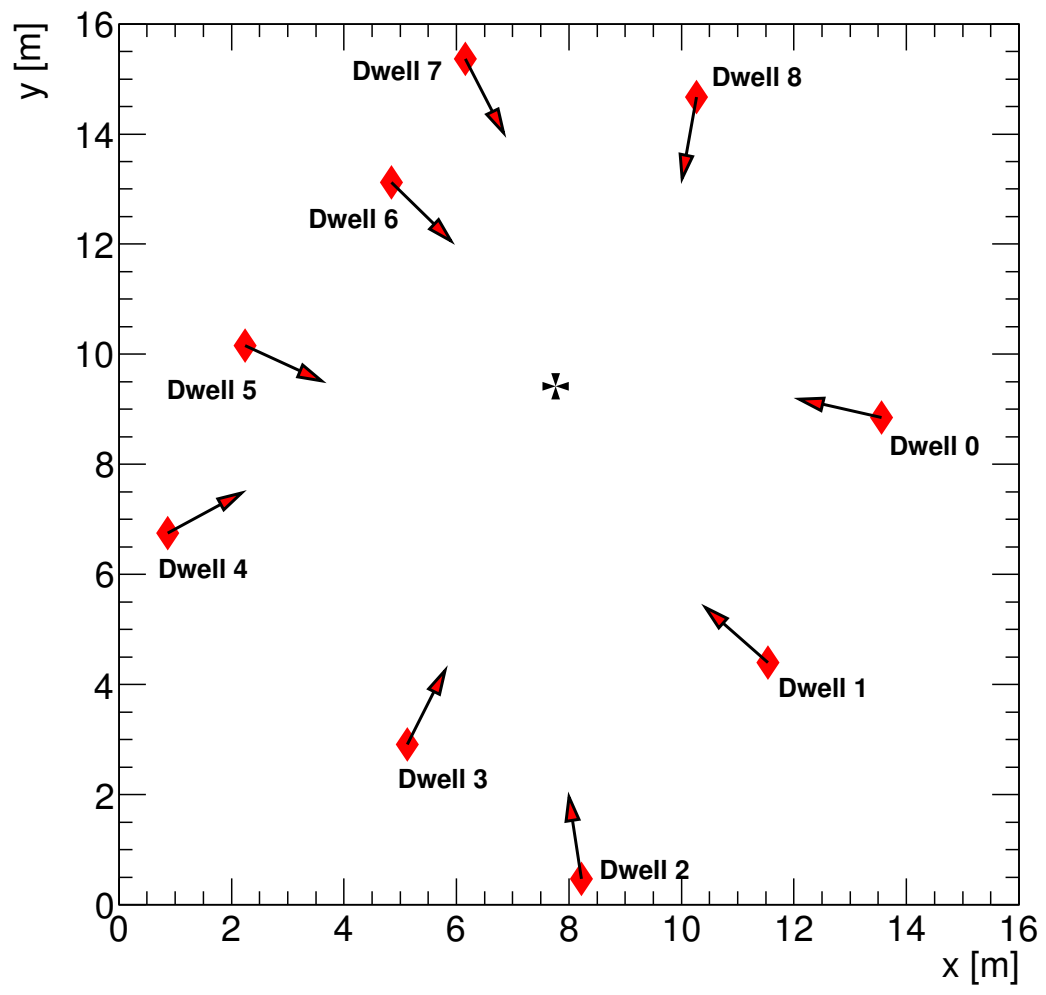

Figure 5.1: Schematic representation of the experimental point-source measurement scenario. Red diamonds mark the locations of the dwell points, while the accompanying red arrows indicate the measured orientations of the detector. The black cross marks the location of the point source.

The 3x3-module SCoTSS detector was mounted on a wheeled cart for transportation. While mounted on the cart, the central plane of the sensitive material of the detector sat $15.4 \mathrm{~cm}$ below the elevation of the point source. The detector was manually positioned at each dwell point, and data were collected for a period of 45 seconds. A 64-channel Ouster OS1 light detection and ranging (LIDAR) sensor* was mounted on top of the 3x3-module SCoTSS detector to provide directional information, the procedure for which is explained further below. The LIDAR unit

*Produced by Ouster in San Francisco. 
has a maximum range of $120 \mathrm{~m}$ and samples $1,310,720$ points per second to form a point-cloud map of the environment with a single-shot precision between $0.7 \mathrm{~cm}$ and $5 \mathrm{~cm}$. The LIDAR unit was positioned $32 \mathrm{~cm}$ above the central plane of the sensitive material of the detector and $20 \mathrm{~cm}$ behind the center of the scatter plane. Figure 5.2 shows the 3x3-module SCoTSS detector with accompanying LIDAR unit mounted on top of the wheeled cart, with image components annotated for clarity.

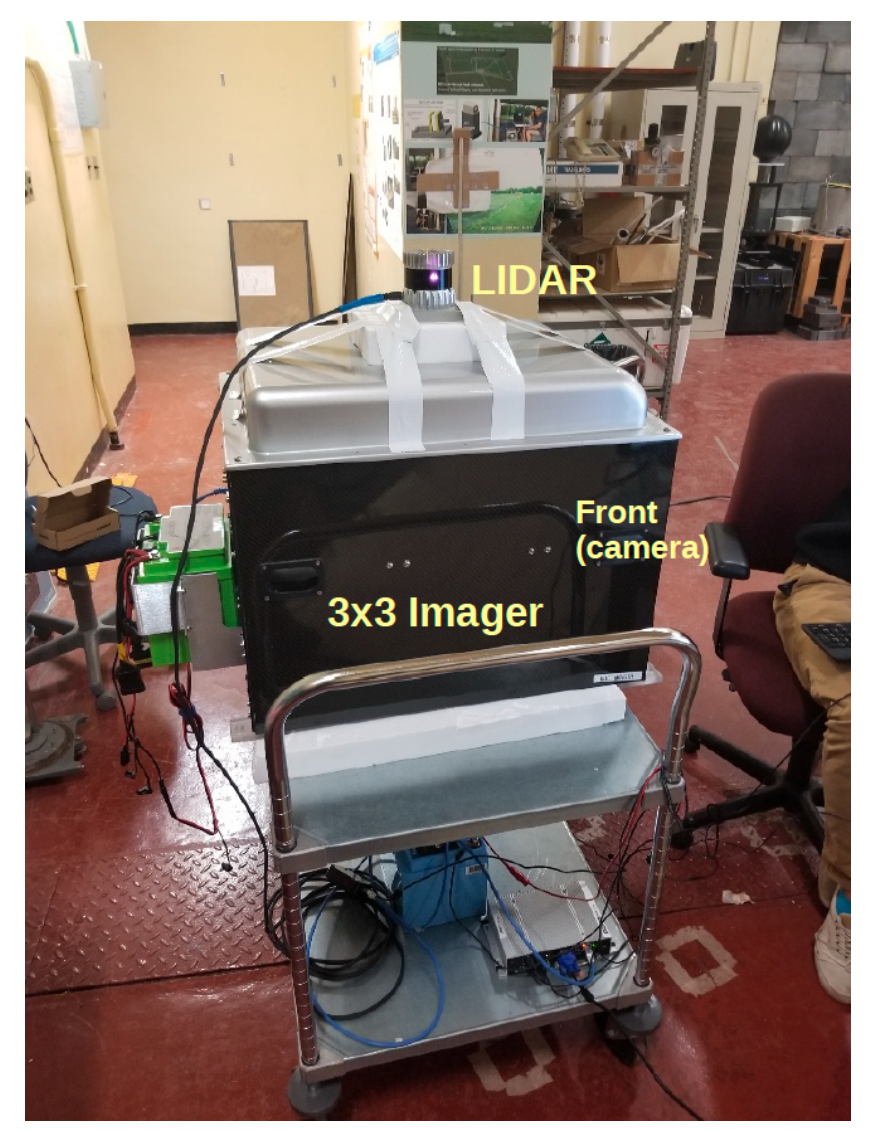

Figure 5.2: LIDAR and 3x3-module SCoTSS detector used for the experimental point-source measurement. The detector sits on top of the wheeled cart used for transportation. Image courtesy of Dr. Patrick Saull, NRC.

Prior to the measurement of the point source a single NORM background measurement was acquired. This measurement was taken as representative of the background at all nine dwell points. 


\section{Data quality}

Figure 5.3 shows total-energy spectra for two-hit events at dwell point 0. Figure 5.3a shows the spectrum of point-source+NORM background measurements at dwell point 0 in black and the NORM background spectrum in blue, where the background spectrum has been scaled as described in section 4.3 using a lower energy limit of $1.5 \mathrm{MeV}$. Figure 5.3b shows the background-subtracted point-source spectrum measured at dwell point 0, where the inset shows a zoomed-in view past the lower energy limit used in the scaling process. Figures 5.4 to 5.11 show similar spectra for dwell points 1 to 8 .

The NORM background data used in the background-subtraction procedure adequately models the background component at each dwell point. The backgroundsubtracted spectra presented in figures 5.3 to 5.11 are generally in agreement with zero counts past the normalization threshold of $1.5 \mathrm{MeV}$, and clear photopeaks are observable at $662 \mathrm{keV}$ for each dwell point. Dwell points 0 and 1 demonstrate small photopeaks at approximately 1.3 MeV even after background subtraction. These two dwell points were in relatively close proximity to a heavily-shielded Co-60 irradiator; this demonstrates the sensitivity of the 3x3-module SCoTSS detector. The NORM background measurement used in the background-subtraction was acquired at a large distance from the Co-60 source, which accounts for why a slight Co-60 signal remains visible in the spectra measured at dwell points 0 and 1. 


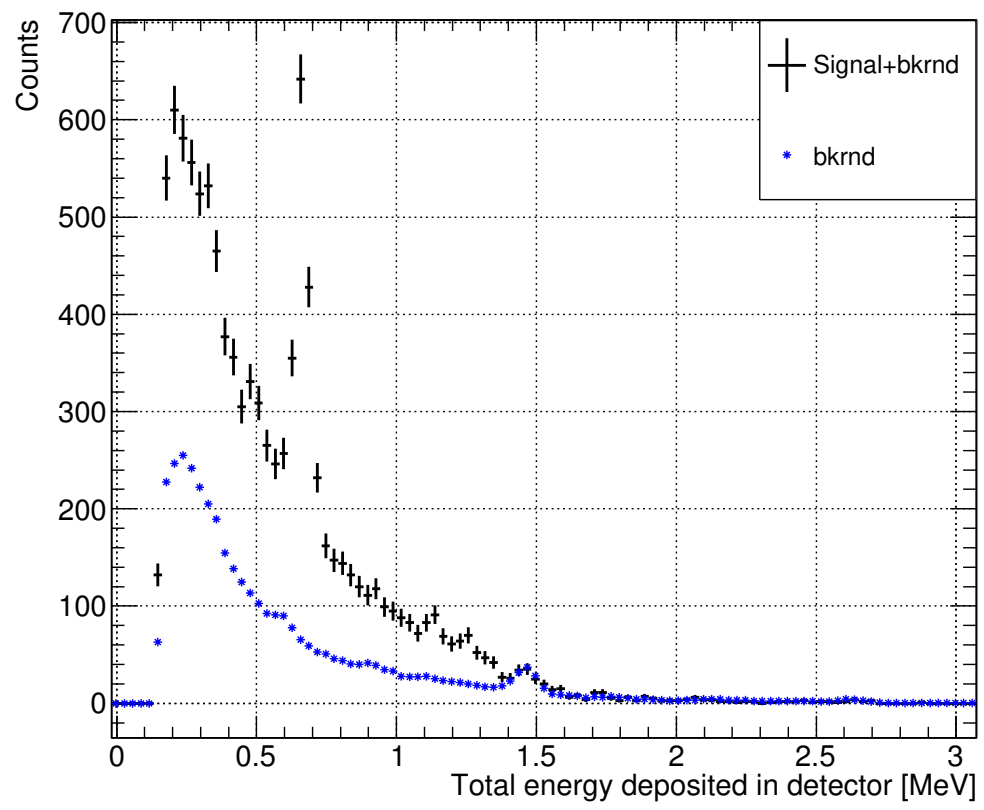

(a)

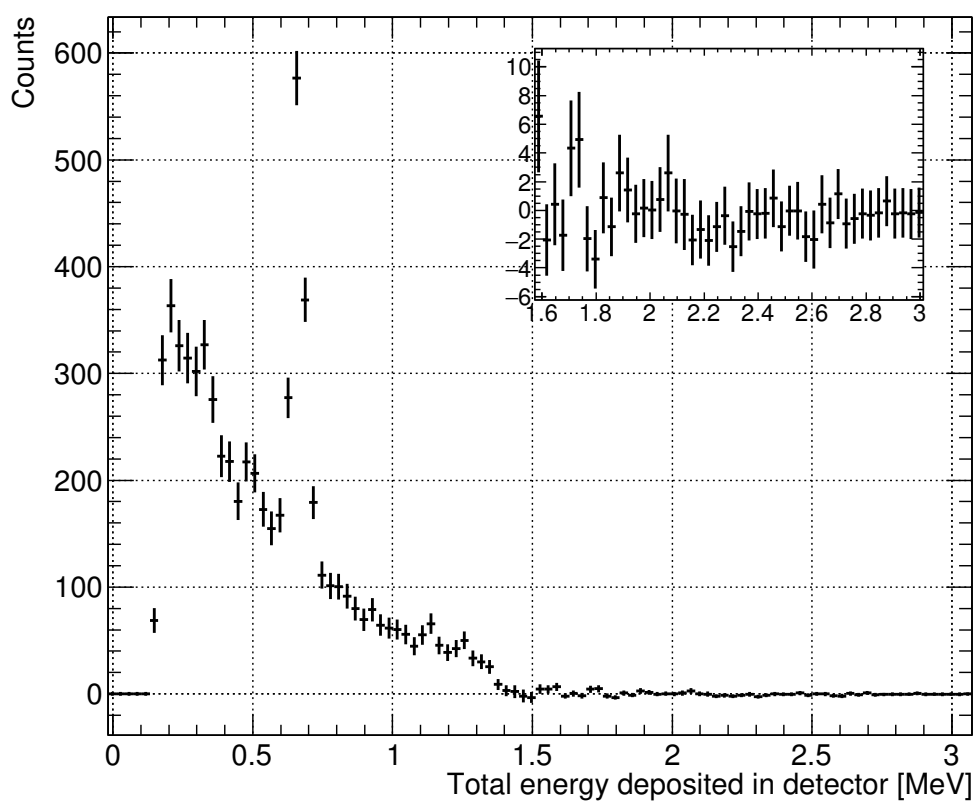

(b)

Figure 5.3: Total-energy spectrum at dwell point 0 in the experimental pointsource measurement. a) Background+source two-hit total-energy spectrum in black, and background-only spectrum in blue (uncertainties are smaller than markers). b) Background-subtracted two-hit total-energy spectrum. Inset shows zoomed-in view of spectrum at high energies. Bins in both subfigures are $30 \mathrm{keV}$. 


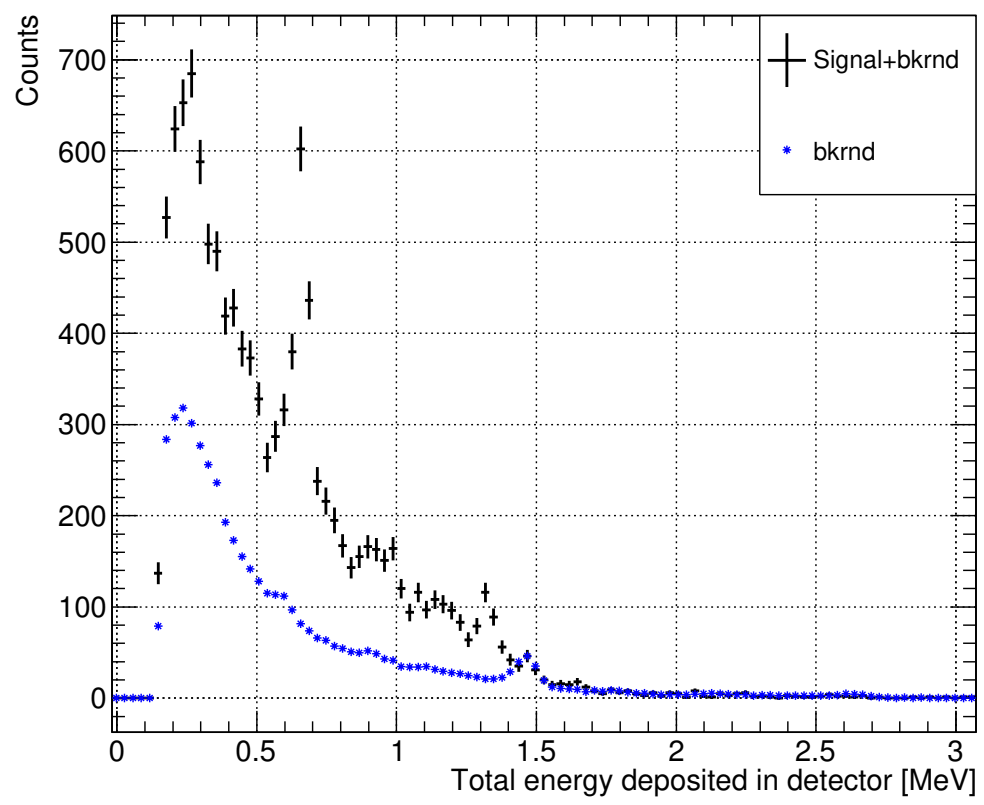

(a)

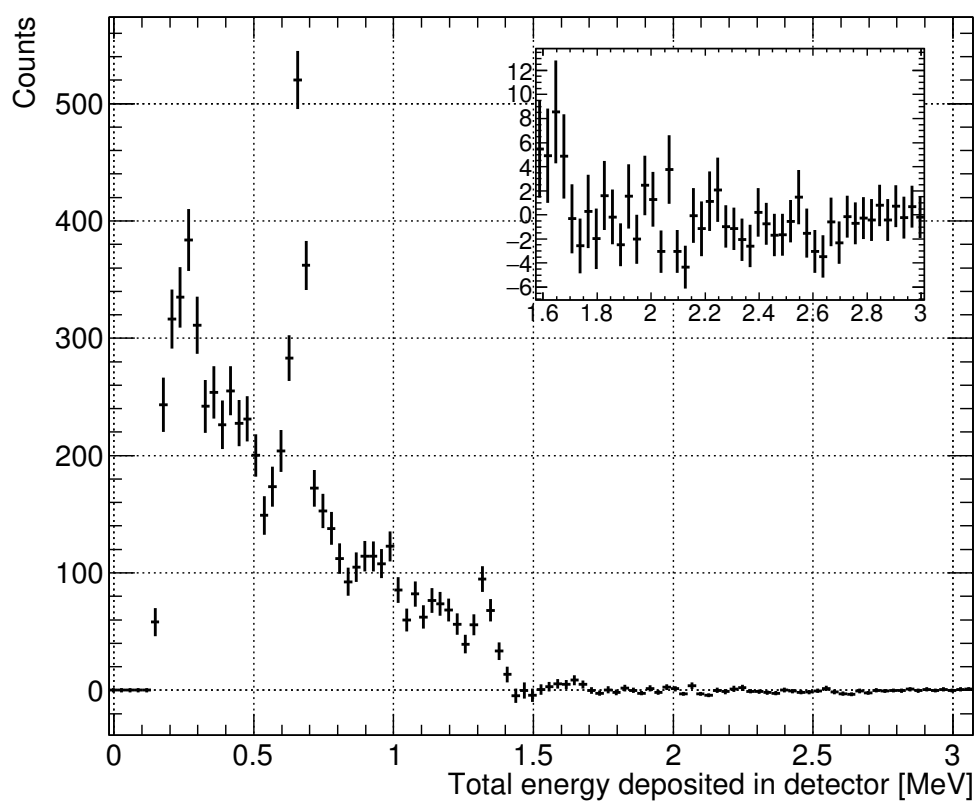

(b)

Figure 5.4: Total-energy spectrum at dwell point 1 in the experimental pointsource measurement. a) Background+source two-hit total-energy spectrum in black, and background-only spectrum in blue (uncertainties are smaller than markers). b) Background-subtracted two-hit total-energy spectrum. Inset shows zoomed-in view of spectrum at high energies. Bins in both subfigures are $30 \mathrm{keV}$. 


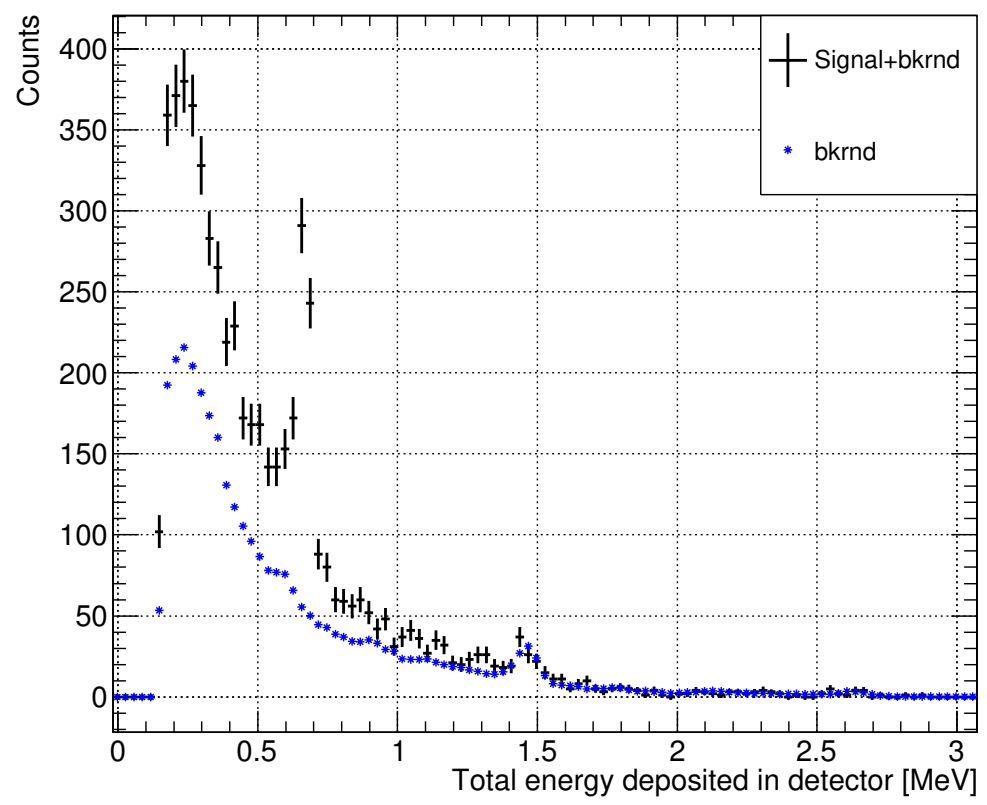

(a)

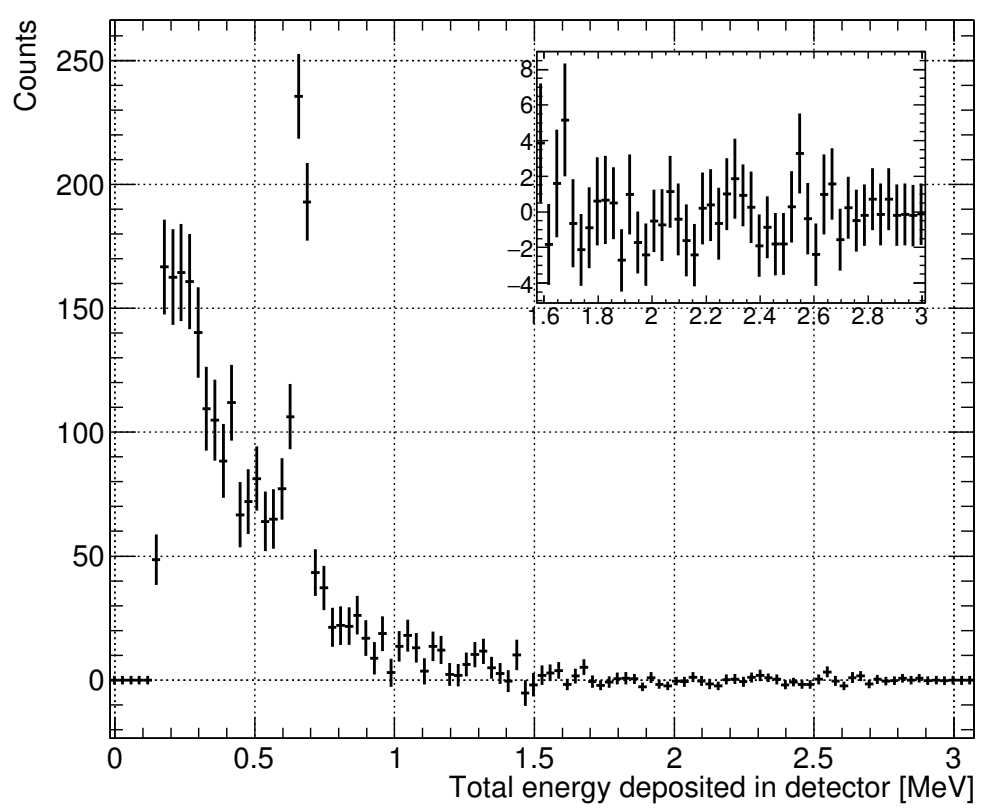

(b)

Figure 5.5: Total-energy spectrum at dwell point 2 in the experimental pointsource measurement. a) Background+source two-hit total-energy spectrum in black, and background-only spectrum in blue (uncertainties are smaller than markers). b) Background-subtracted two-hit total-energy spectrum. Inset shows zoomed-in view of spectrum at high energies. Bins in both subfigures are $30 \mathrm{keV}$. 


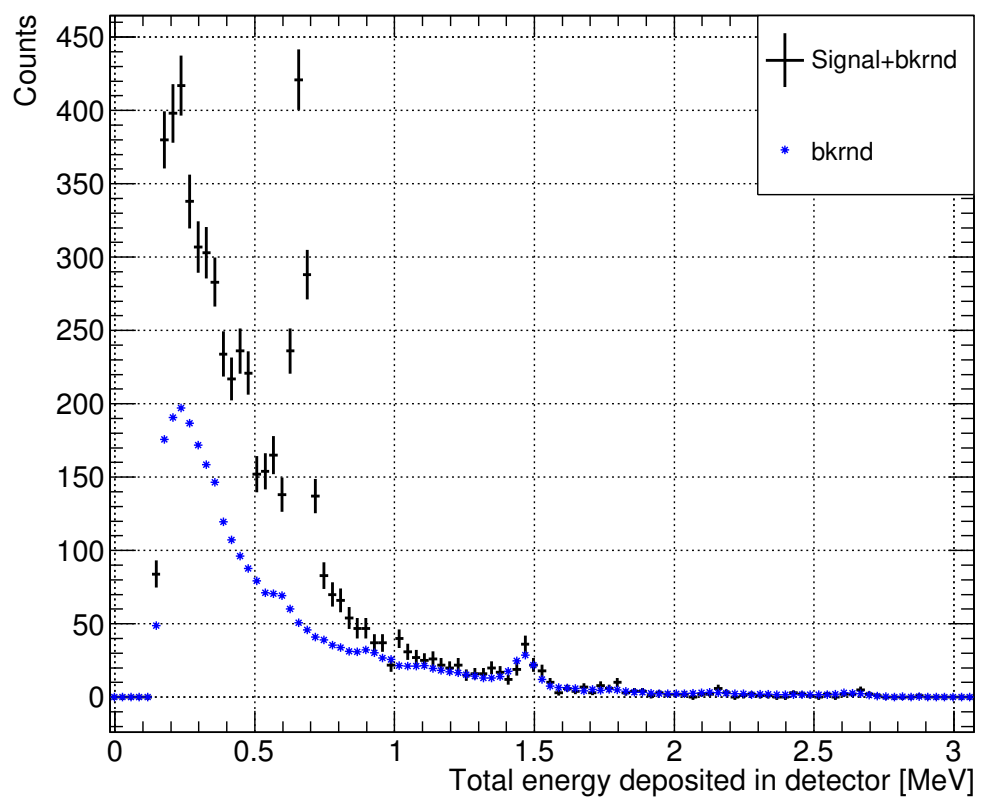

(a)

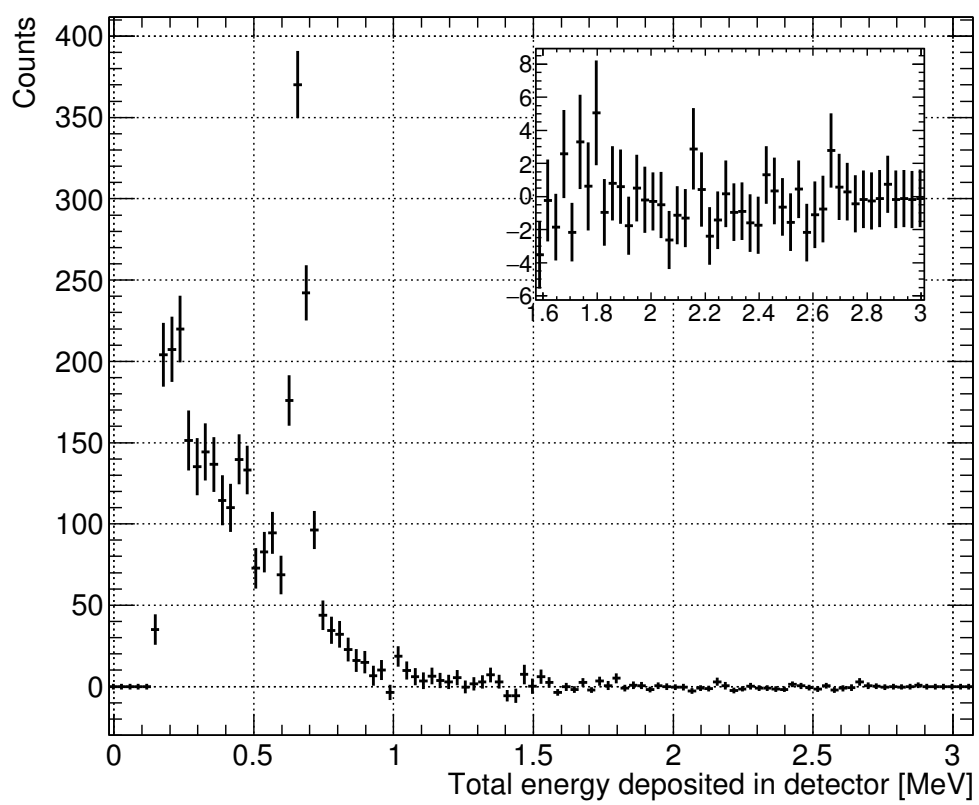

(b)

Figure 5.6: Total-energy spectrum at dwell point 3 in the experimental pointsource measurement. a) Background+source two-hit total-energy spectrum in black, and background-only spectrum in blue (uncertainties are smaller than markers). b) Background-subtracted two-hit total-energy spectrum. Inset shows zoomed-in view of spectrum at high energies. Bins in both subfigures are $30 \mathrm{keV}$. 


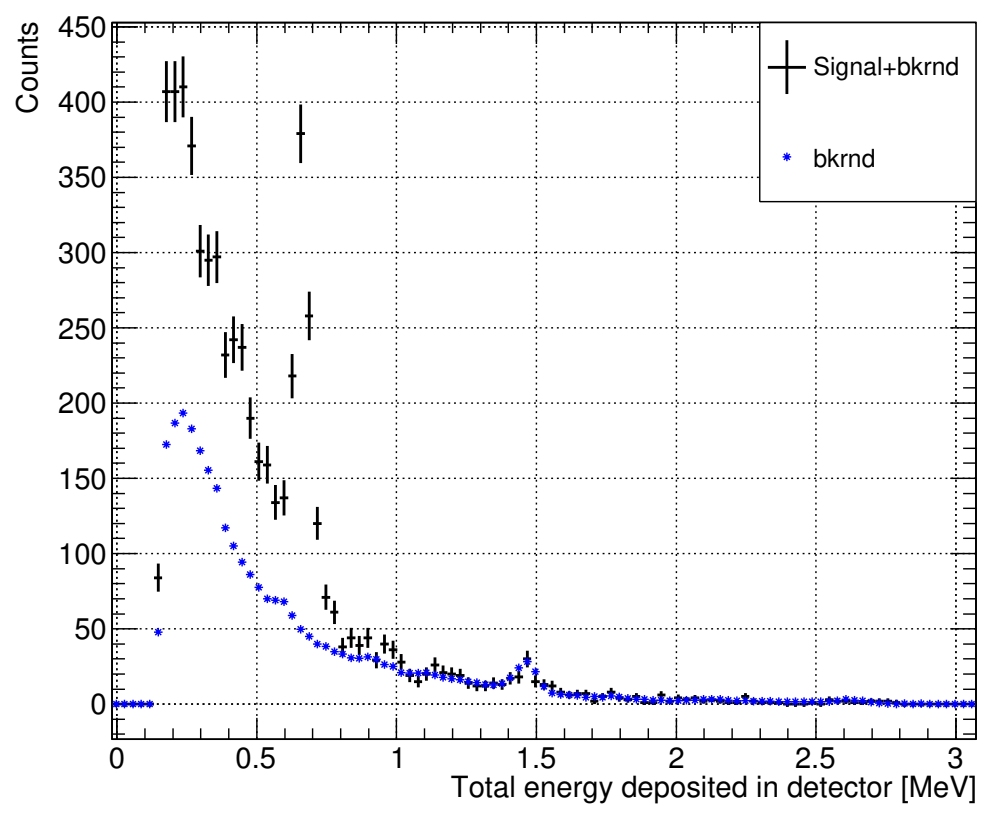

(a)

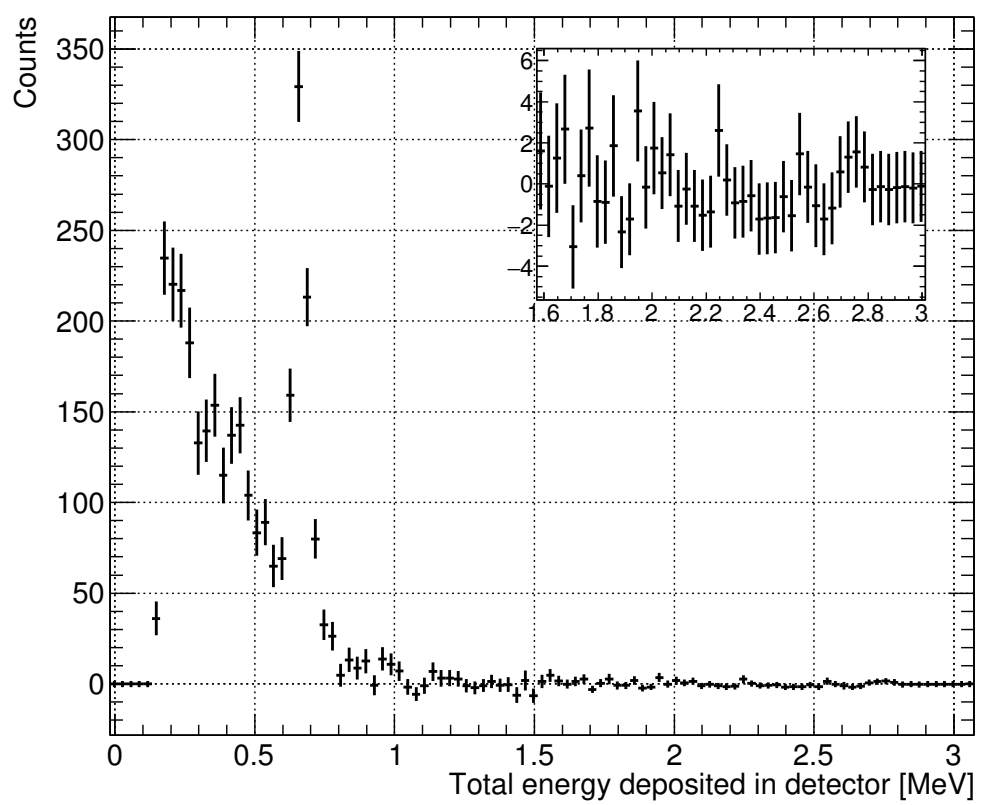

(b)

Figure 5.7: Total-energy spectrum at dwell point 4 in the experimental pointsource measurement. a) Background+source two-hit total-energy spectrum in black, and background-only spectrum in blue (uncertainties are smaller than markers). b) Background-subtracted two-hit total-energy spectrum. Inset shows zoomed-in view of spectrum at high energies. Bins in both subfigures are $30 \mathrm{keV}$. 


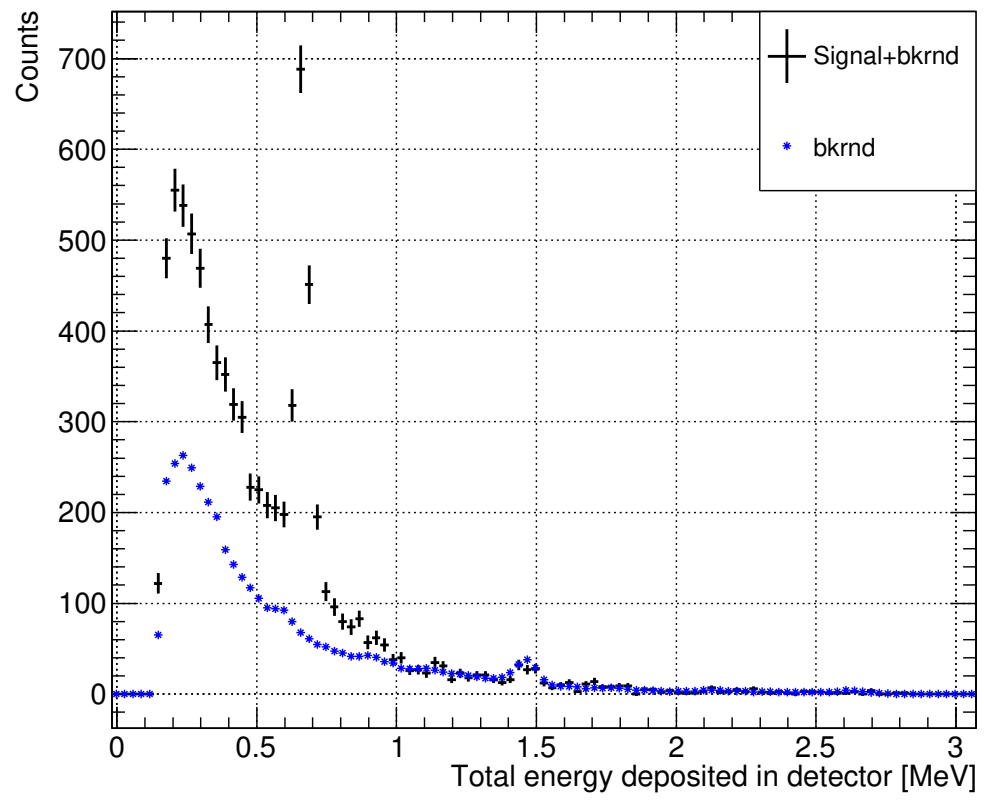

(a)

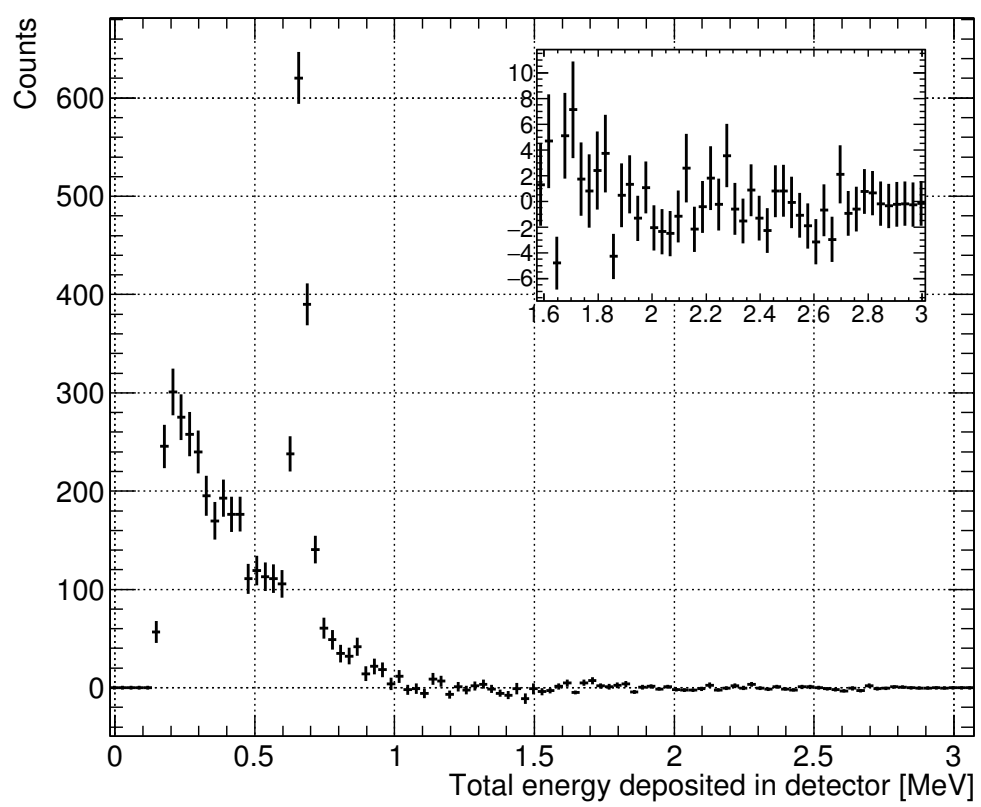

(b)

Figure 5.8: Total-energy spectrum at dwell point 5 in the experimental pointsource measurement. a) Background+source two-hit total-energy spectrum in black, and background-only spectrum in blue (uncertainties are smaller than markers). b) Background-subtracted two-hit total-energy spectrum. Inset shows zoomed-in view of spectrum at high energies. Bins in both subfigures are $30 \mathrm{keV}$. 


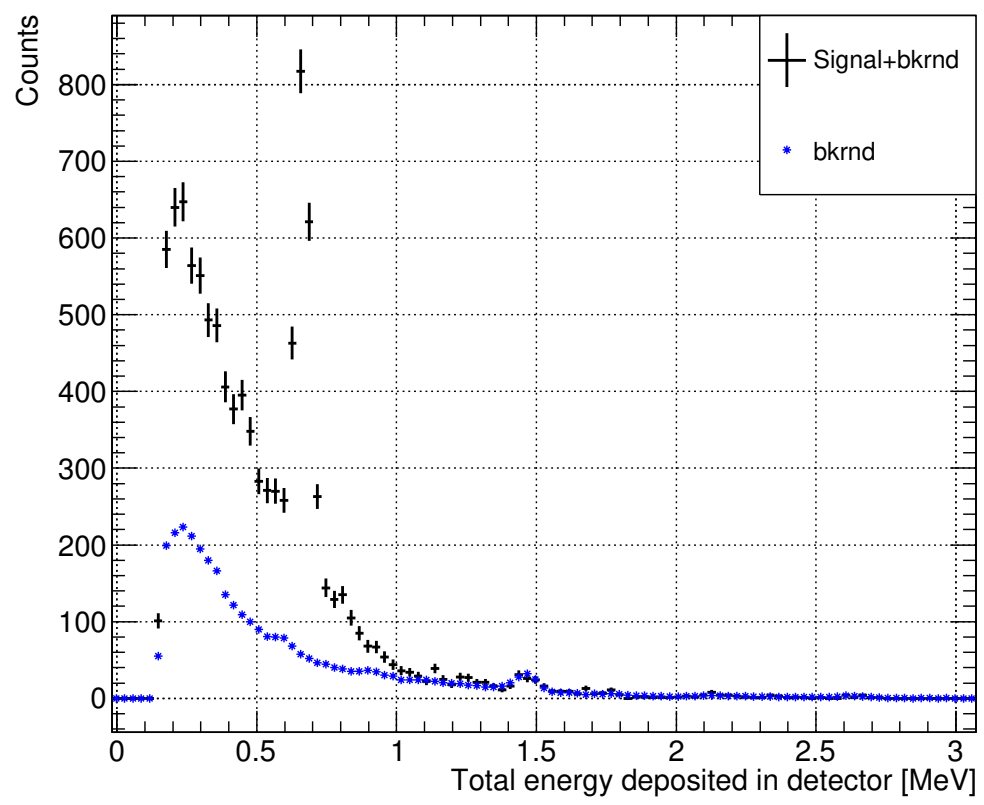

(a)

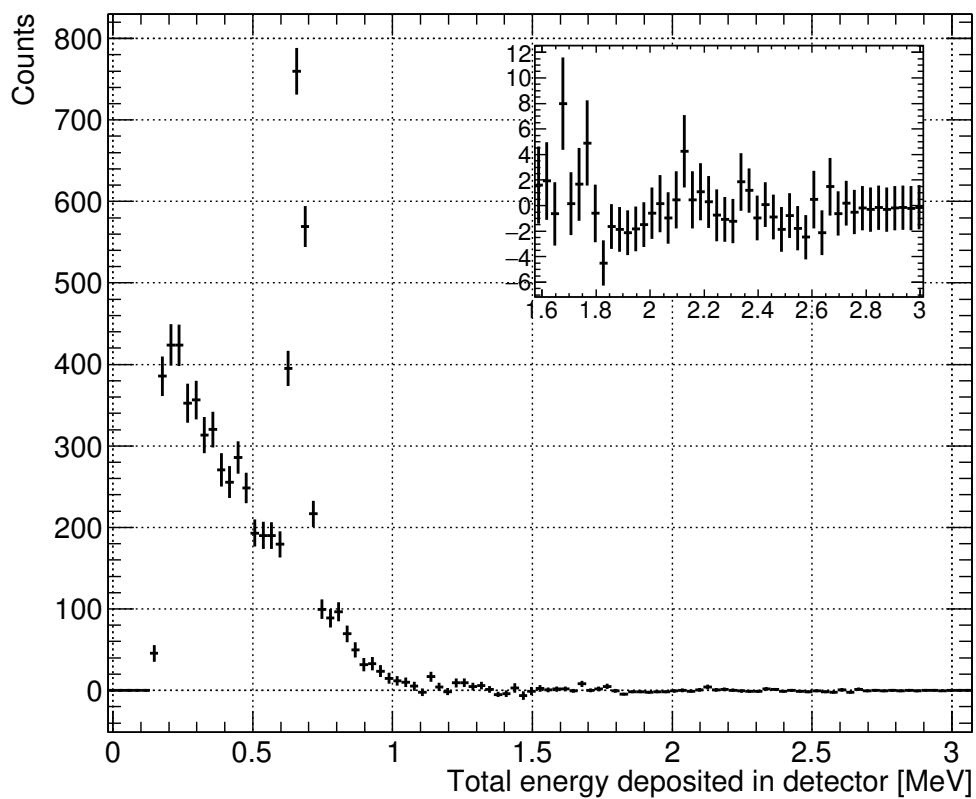

(b)

Figure 5.9: Total-energy spectrum at dwell point 6 in the experimental pointsource measurement. a) Background+source two-hit total-energy spectrum in black, and background-only spectrum in blue (uncertainties are smaller than markers). b) Background-subtracted two-hit total-energy spectrum. Inset shows zoomed-in view of spectrum at high energies. Bins in both subfigures are $30 \mathrm{keV}$. 


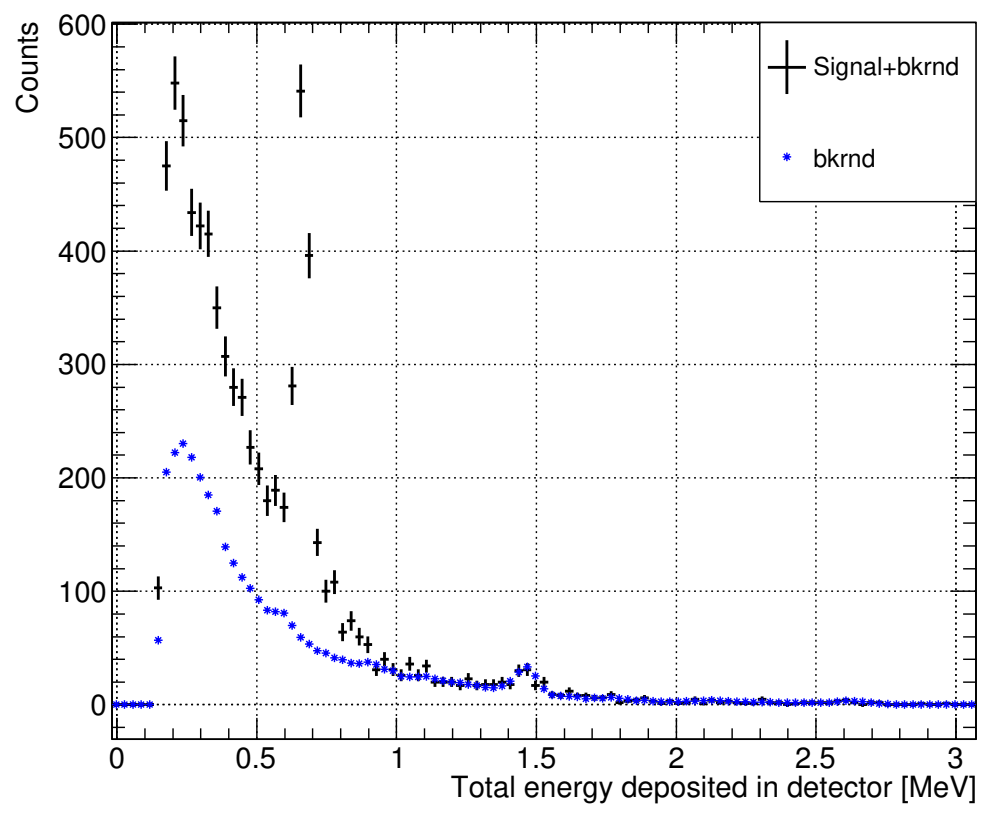

(a)

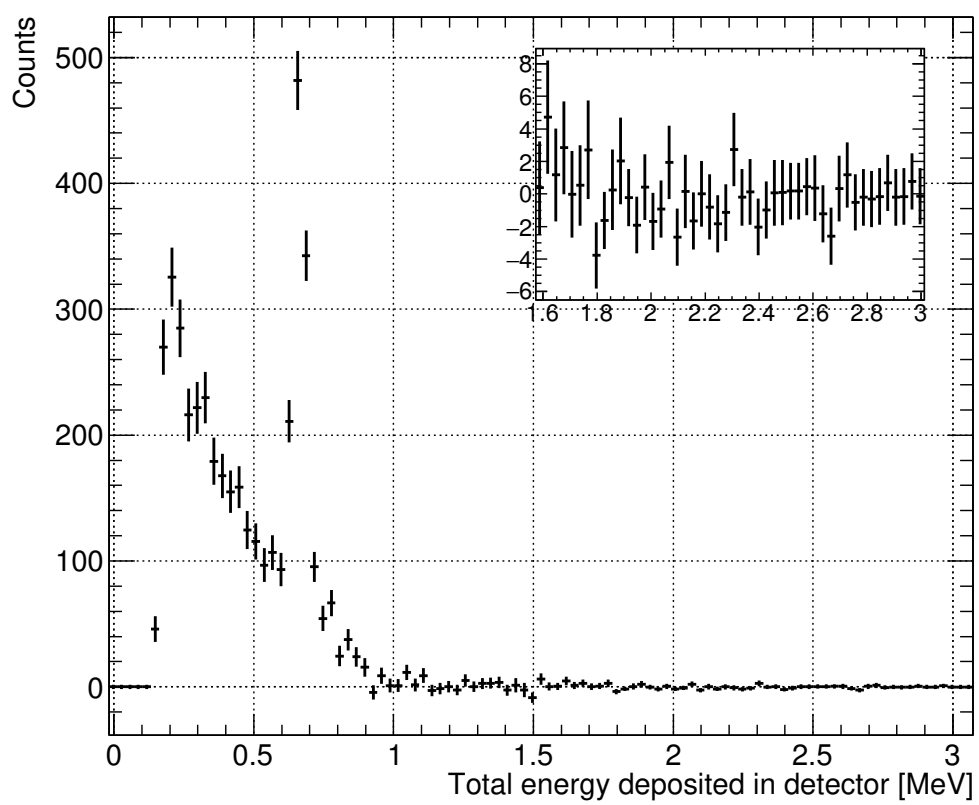

(b)

Figure 5.10: Total-energy spectrum at dwell point 7 in the experimental pointsource measurement. a) Background+source two-hit total-energy spectrum in black, and background-only spectrum in blue (uncertainties are smaller than markers). b) Background-subtracted two-hit total-energy spectrum. Inset shows zoomed-in view of spectrum at high energies. Bins in both subfigures are $30 \mathrm{keV}$. 


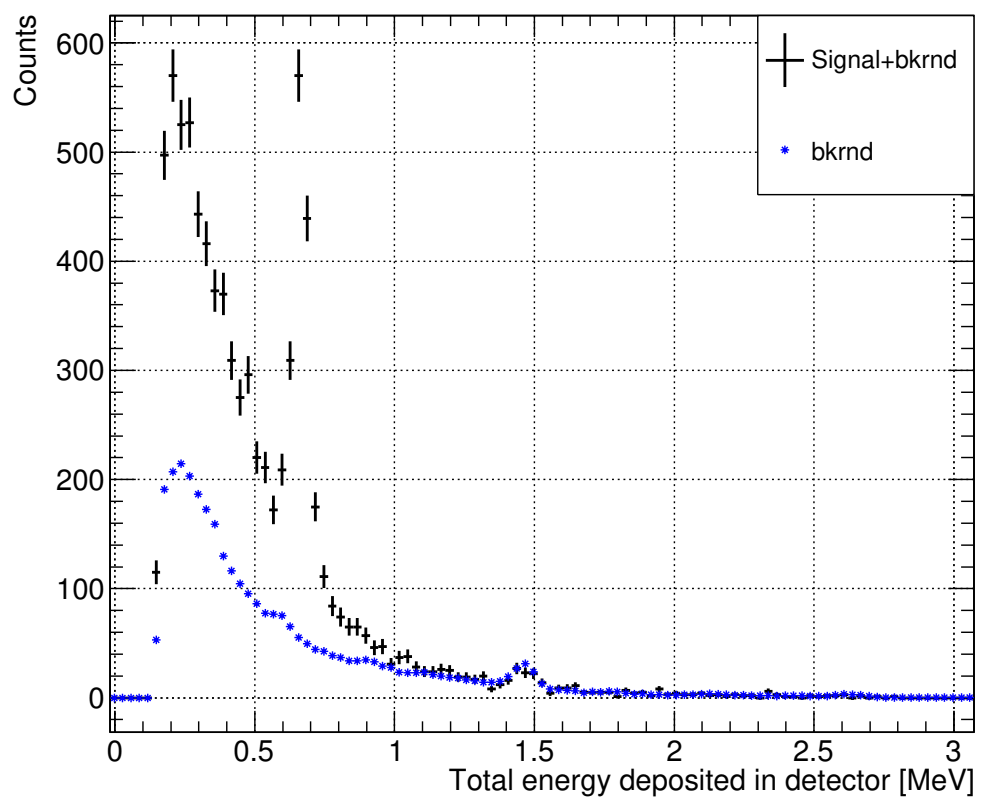

(a)

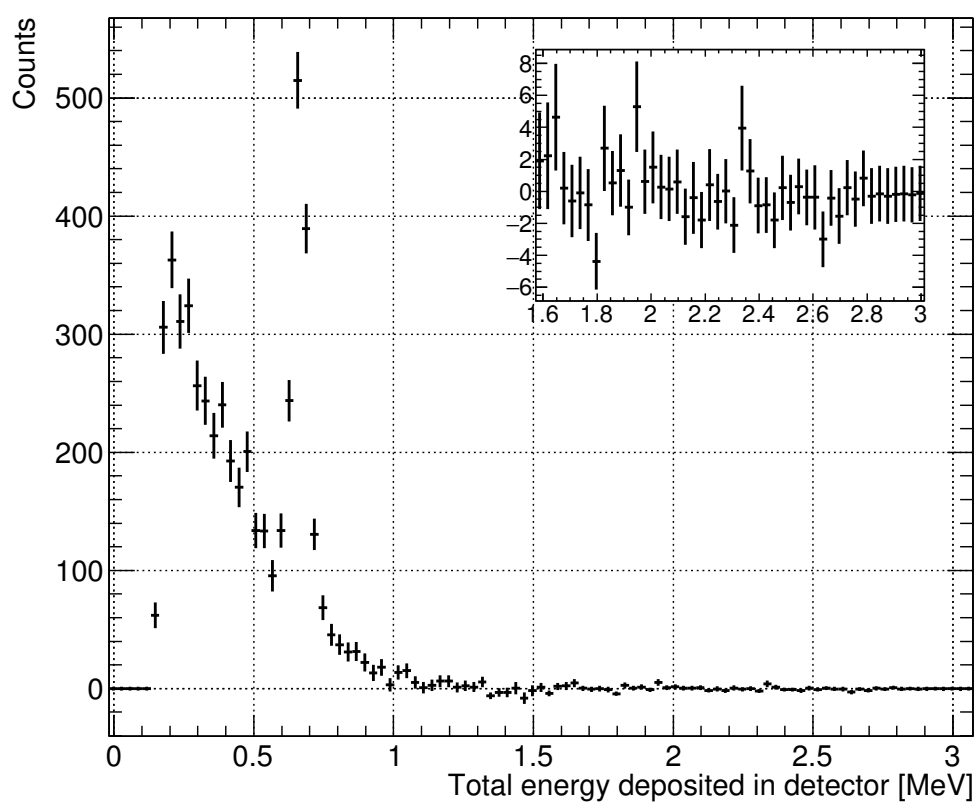

(b)

Figure 5.11: Total-energy spectrum at dwell point 8 in the experimental pointsource measurement. a) Background+source two-hit total-energy spectrum in black, and background-only spectrum in blue (uncertainties are smaller than markers). b) Background-subtracted two-hit total-energy spectrum. Inset shows zoomed-in view of spectrum at high energies. Bins in both subfigures are $30 \mathrm{keV}$. 


\section{Event selection}

The event-selection criteria were chosen following the principles outlined in section 4.2, and are presented in table 5.1.

Table 5.1: Energy cuts used with the 3x3-module SCoTSS detector in event selection for the 662 keV photons in the experimental point-source measurement.

\begin{tabular}{l|l} 
Selection criteria & Energy requirements \\
\hline Preselection and noise rejection & $\begin{array}{l}E_{1}>15 \mathrm{keV} \text { and } E_{2}>25 \mathrm{keV} \\
\text { and } E_{1}+E_{2}>150 \mathrm{keV}\end{array}$ \\
$\begin{array}{l}\text { Photopeak selection } \\
\begin{array}{l}\text { Back-scatter rejection and } \\
\text { geometric selection }\end{array}\end{array}$ & $E_{1}<160 \mathrm{keV}$
\end{tabular}

Table 5.2 shows the number of events passing the event selection criteria given in table 5.1 at each dwell point. In total, there were 4772 events accepted across all of the dwell points.

Table 5.2: Number of events passing the selection criteria at each dwell point in the experimental point-source measurement.

\begin{tabular}{c|c} 
Dwell point & Events passing cuts \\
\hline 0 & 588 \\
1 & 546 \\
2 & 310 \\
3 & 420 \\
4 & 387 \\
5 & 592 \\
6 & 833 \\
7 & 522 \\
8 & 574
\end{tabular}

\section{Individual dwell-point Compton gamma images}

Figure 5.12a shows an overlay of the gamma image on an optical image acquired during the experimental measurement at dwell point 0, where the contours of the gamma image shown are as they existed during the experimental trial and do not include background subtraction. To co-register the gamma image to the optical im- 
age, the Compton cone information has been adjusted to agree with the coordinate system of the optical photograph. Figure $5.12 \mathrm{~b}$ shows a gamma image made from the experimental data acquired at dwell point 0 in post-processing and includes background subtraction. This gamma image represents the information from dwell point 0 that contributes to the tomographic reconstruction of the data discussed later in this chapter. Figures 5.13 to 5.20 show analogous images for dwell points 1 to 8 . The contours overlaid on the optical images accurately localize the point source, which is not directly visible on the scale of the image. These point-of-view gamma images are not directly used for the tomographic reconstruction methodologies that are presented below in this chapter. However, they may be used in-situ to localize the radioactive source in the field of view of the detector and to guide the operator.

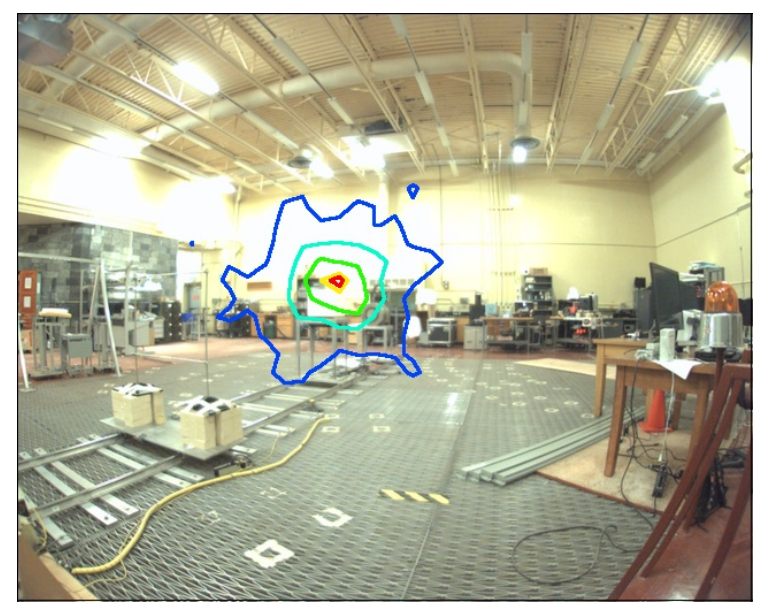

(a)

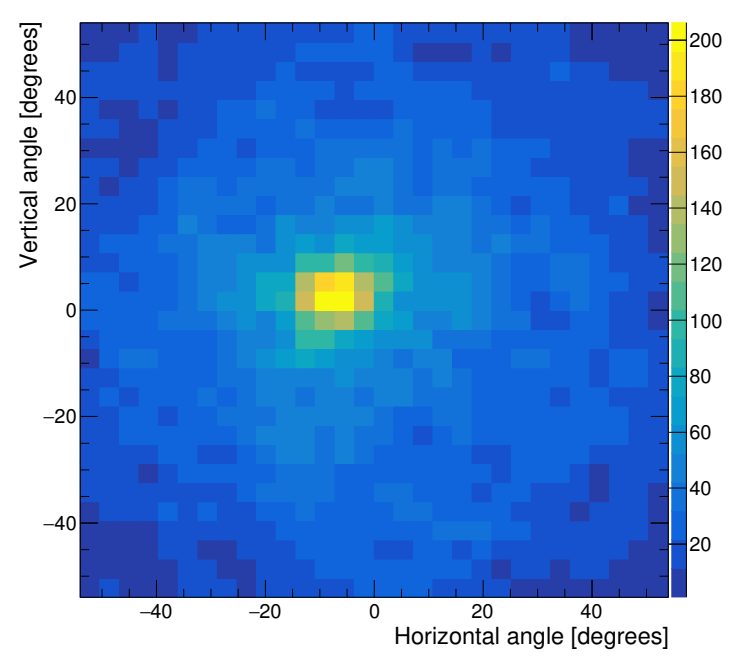

(b)

Figure 5.12: Field-of-view gamma image at dwell point 0 in the experimental pointsource measurement. a) Field-of-view image overlaid on optical image, as acquired during the experiment. b) Background-subtracted field-of-view image generated in post-processing. 


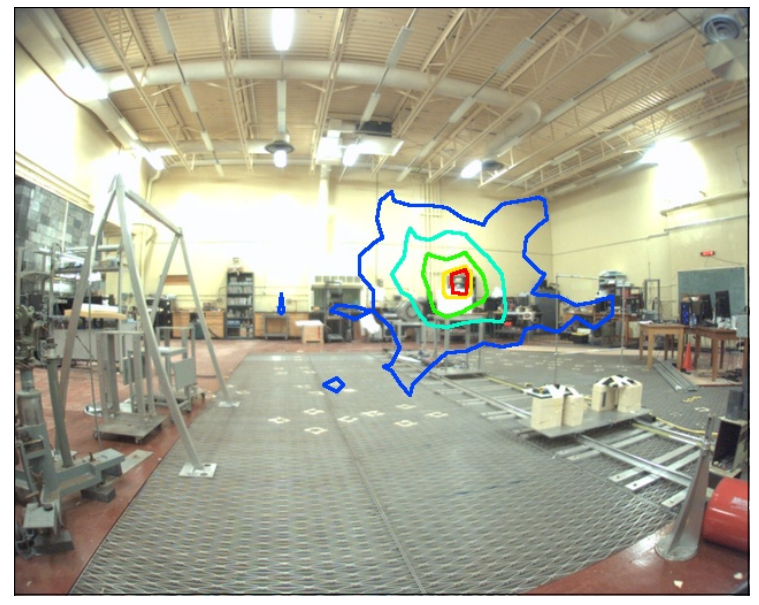

(a)

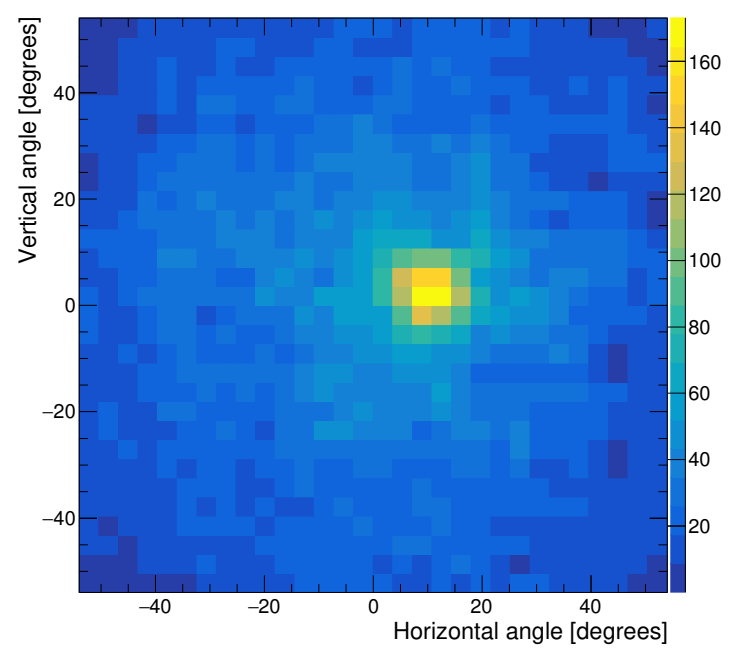

(b)

Figure 5.13: Field-of-view gamma image at dwell point 1 in the experimental pointsource measurement. a) Field-of-view image overlaid on optical image, as acquired during the experiment. b) Background-subtracted field-of-view image generated in post-processing.

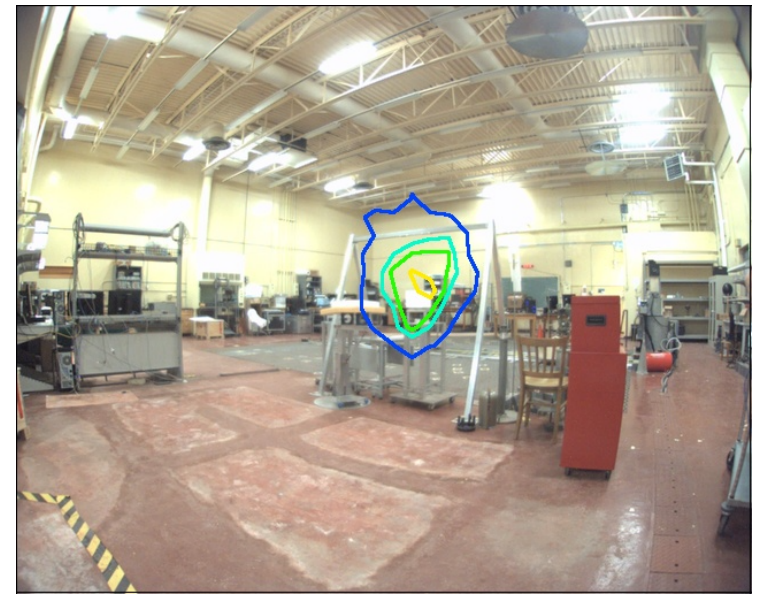

(a)

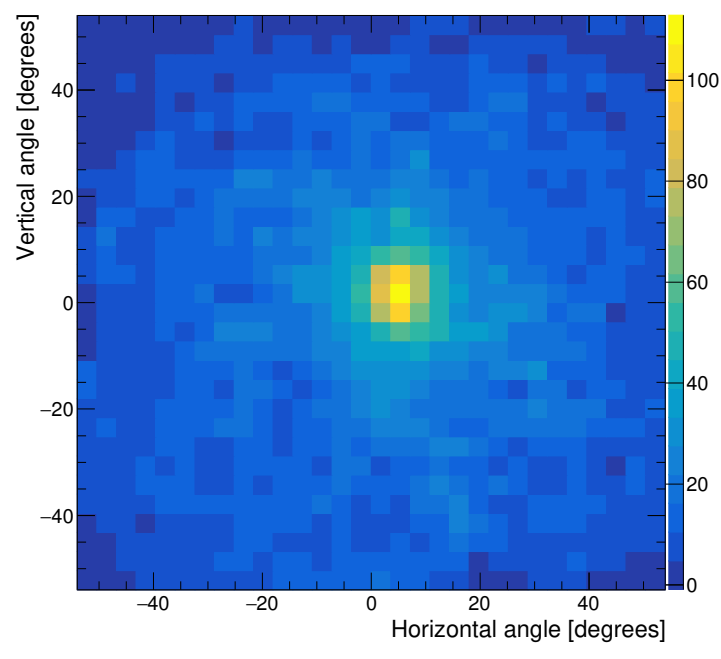

(b)

Figure 5.14: Field-of-view gamma image at dwell point 2 in the experimental pointsource measurement. a) Field-of-view image overlaid on optical image, as acquired during the experiment. b) Background-subtracted field-of-view image generated in post-processing. 


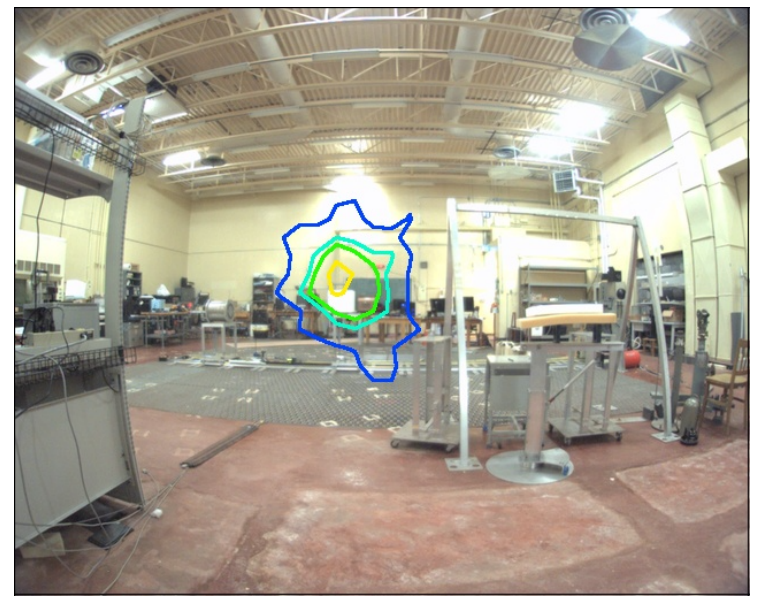

(a)

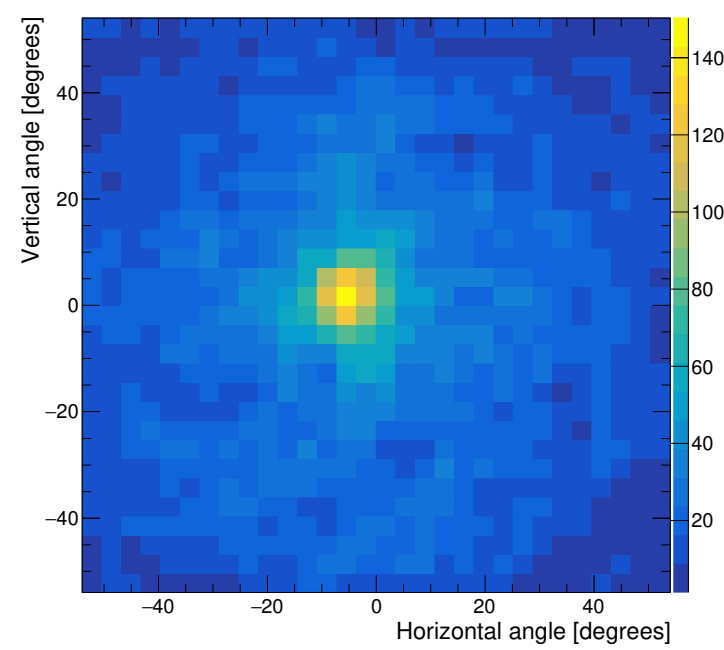

(b)

Figure 5.15: Field-of-view gamma image at dwell point 3 in the experimental pointsource measurement. a) Field-of-view image overlaid on optical image, as acquired during the experiment. b) Background-subtracted field-of-view image generated in post-processing.

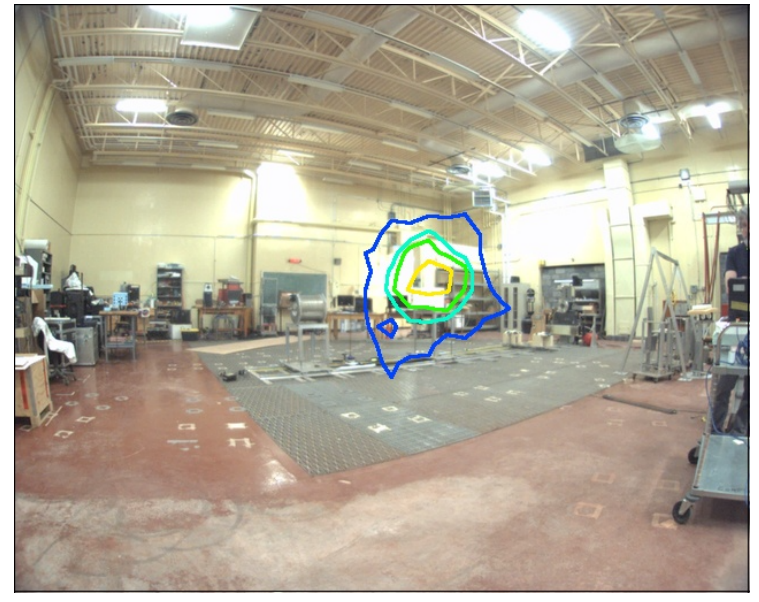

(a)

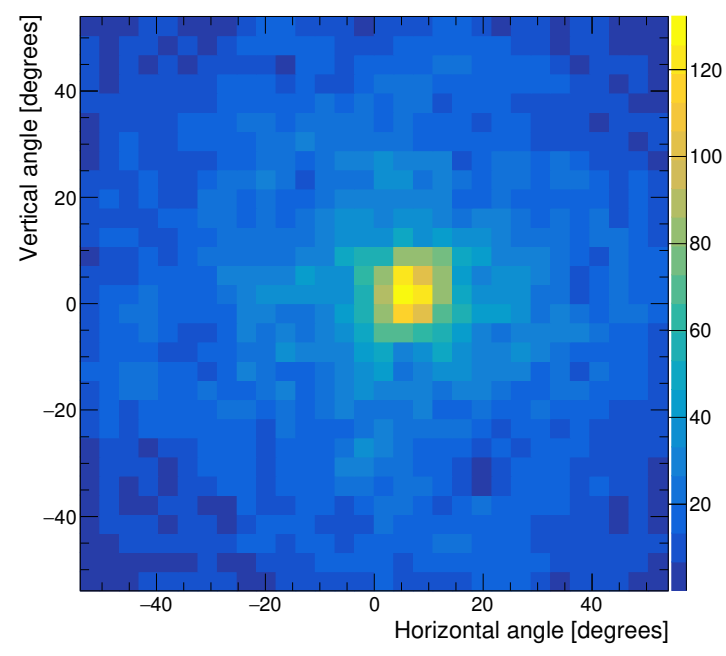

(b)

Figure 5.16: Field-of-view gamma image at dwell point 4 in the experimental pointsource measurement. a) Field-of-view image overlaid on optical image, as acquired during the experiment. b) Background-subtracted field-of-view image generated in post-processing. 


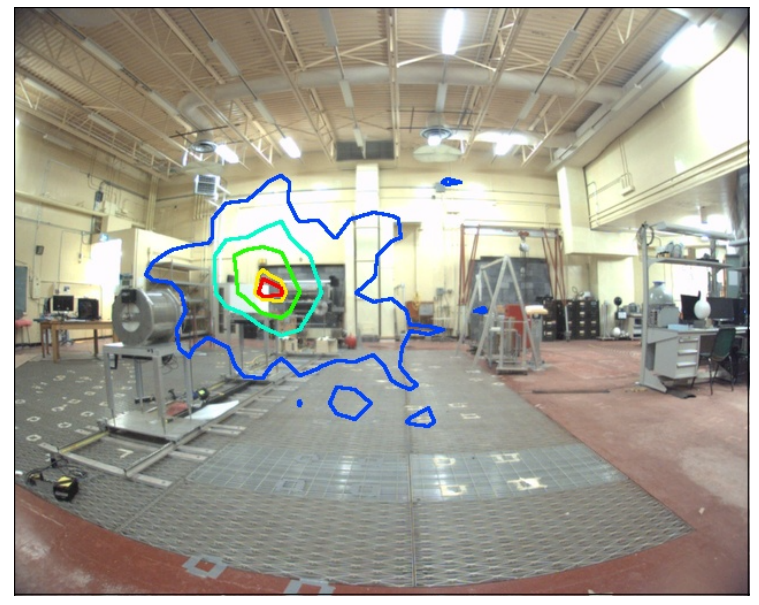

(a)

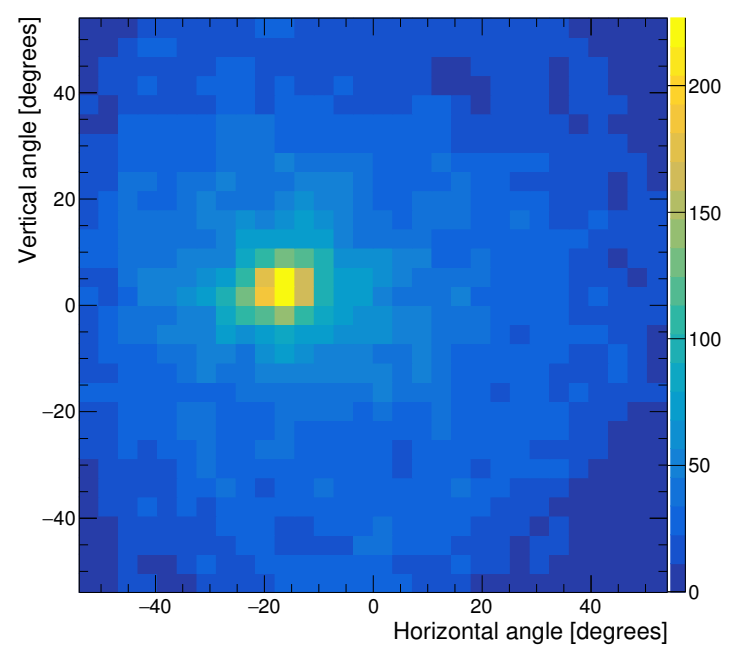

(b)

Figure 5.17: Field-of-view gamma image at dwell point 5 in the experimental pointsource measurement. a) Field-of-view image overlaid on optical image, as acquired during the experiment. b) Background-subtracted field-of-view image generated in post-processing.

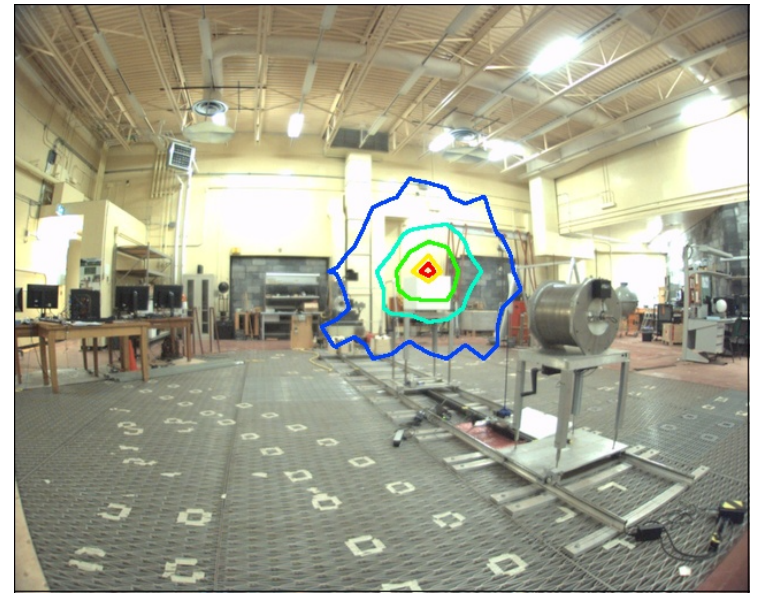

(a)

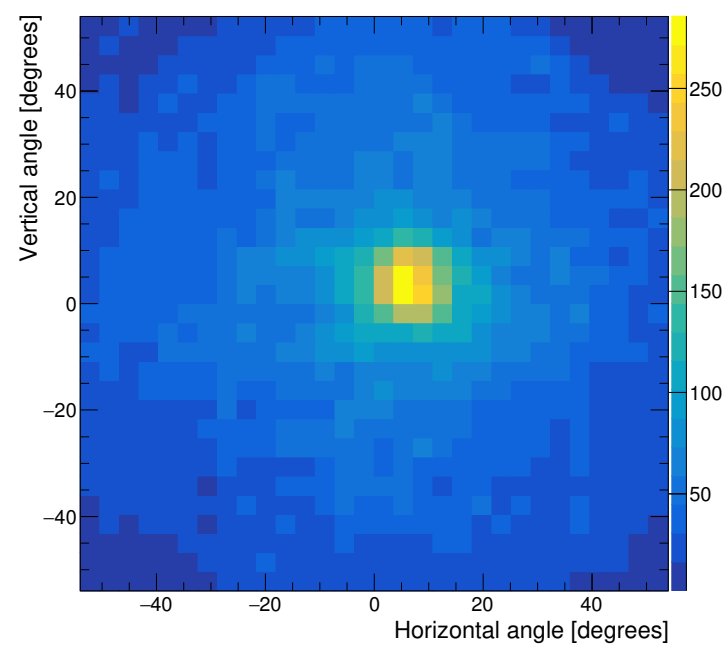

(b)

Figure 5.18: Field-of-view gamma image at dwell point 6 in the experimental pointsource measurement. a) Field-of-view image overlaid on optical image, as acquired during the experiment. b) Background-subtracted field-of-view image generated in post-processing. 


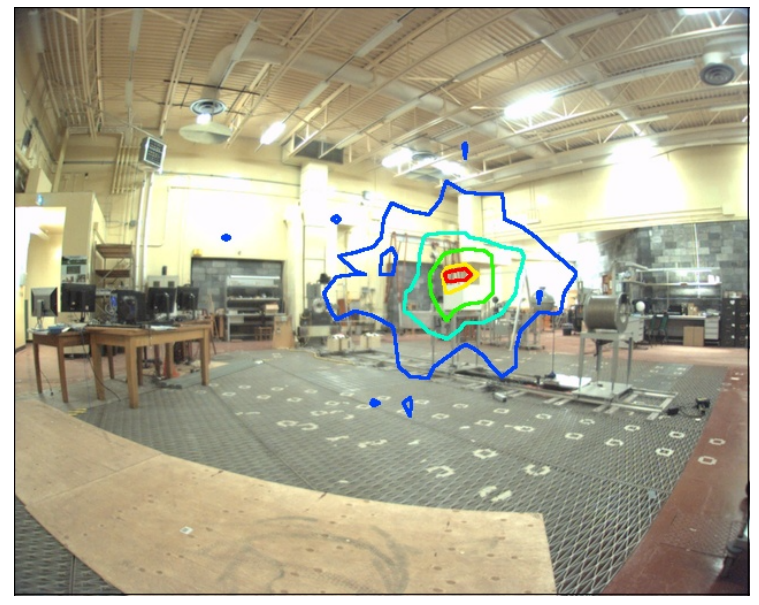

(a)

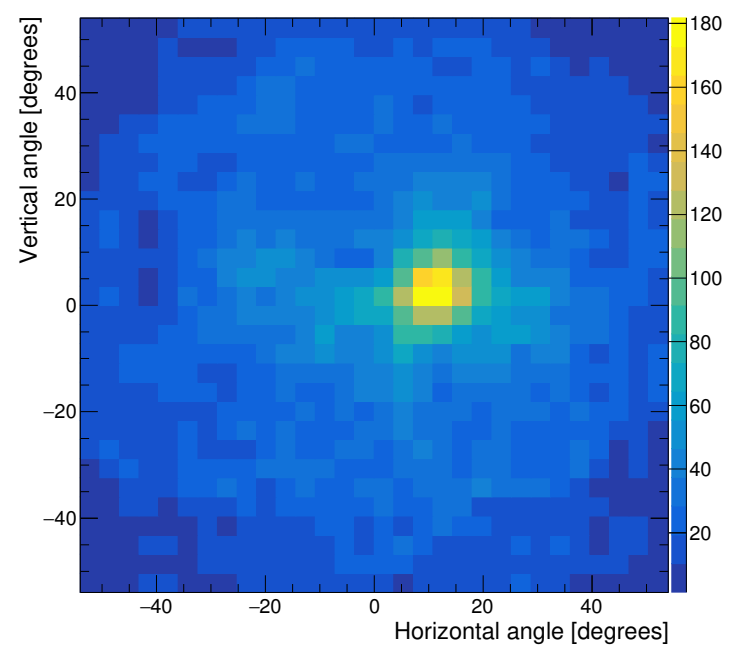

(b)

Figure 5.19: Field-of-view gamma image at dwell point 7 in the experimental pointsource measurement. a) Field-of-view image overlaid on optical image, as acquired during the experiment. b) Background-subtracted field-of-view image generated in post-processing.

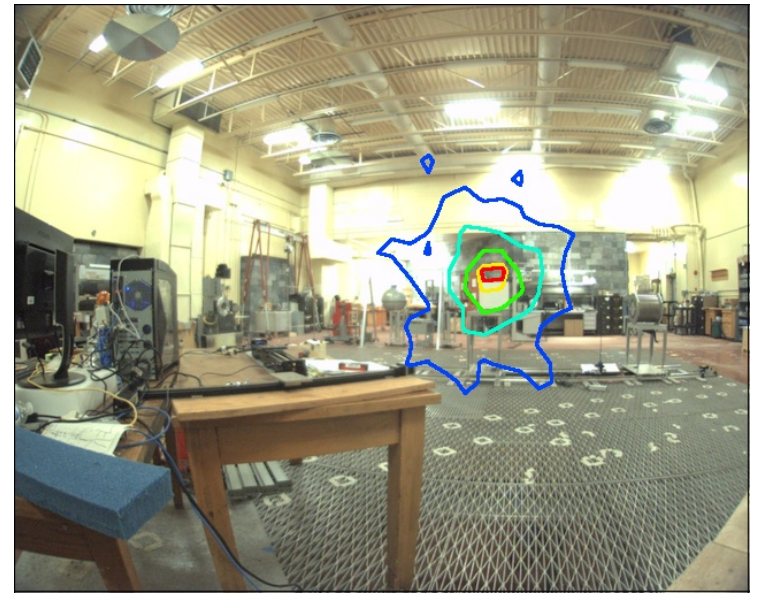

(a)

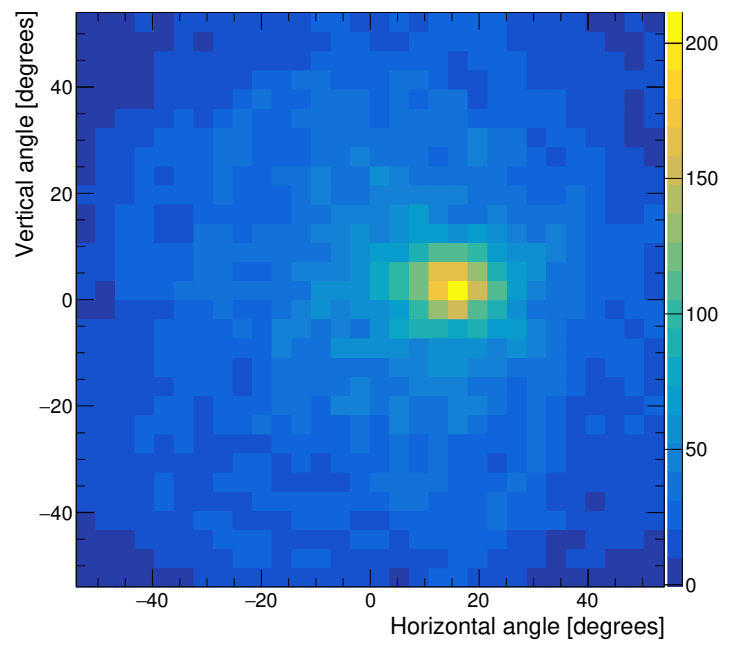

(b)

Figure 5.20: Field-of-view gamma image at dwell point 8 in the experimental pointsource measurement. a) Field-of-view image overlaid on optical image, as acquired during the experiment. b) Background-subtracted field-of-view image generated in post-processing. 


\subsubsection{Estimating the position and orientation of the $3 \times 3$ - module SCoTSS detector}

The LIDAR unit paired with the 3x3-module SCoTSS detector continuously populated a point-cloud representation of the laboratory as it was transported with the 3x3-module SCoTSS detector to each dwell point. Figure 5.21 provides a representative point-cloud map of the laboratory space at the NRC within which the experiment was performed. Figure 5.21 was generated by translating the combined LIDAR and detector system through the laboratory space, combining the point-cloud information to form a global map of the environment with high spatial precision.

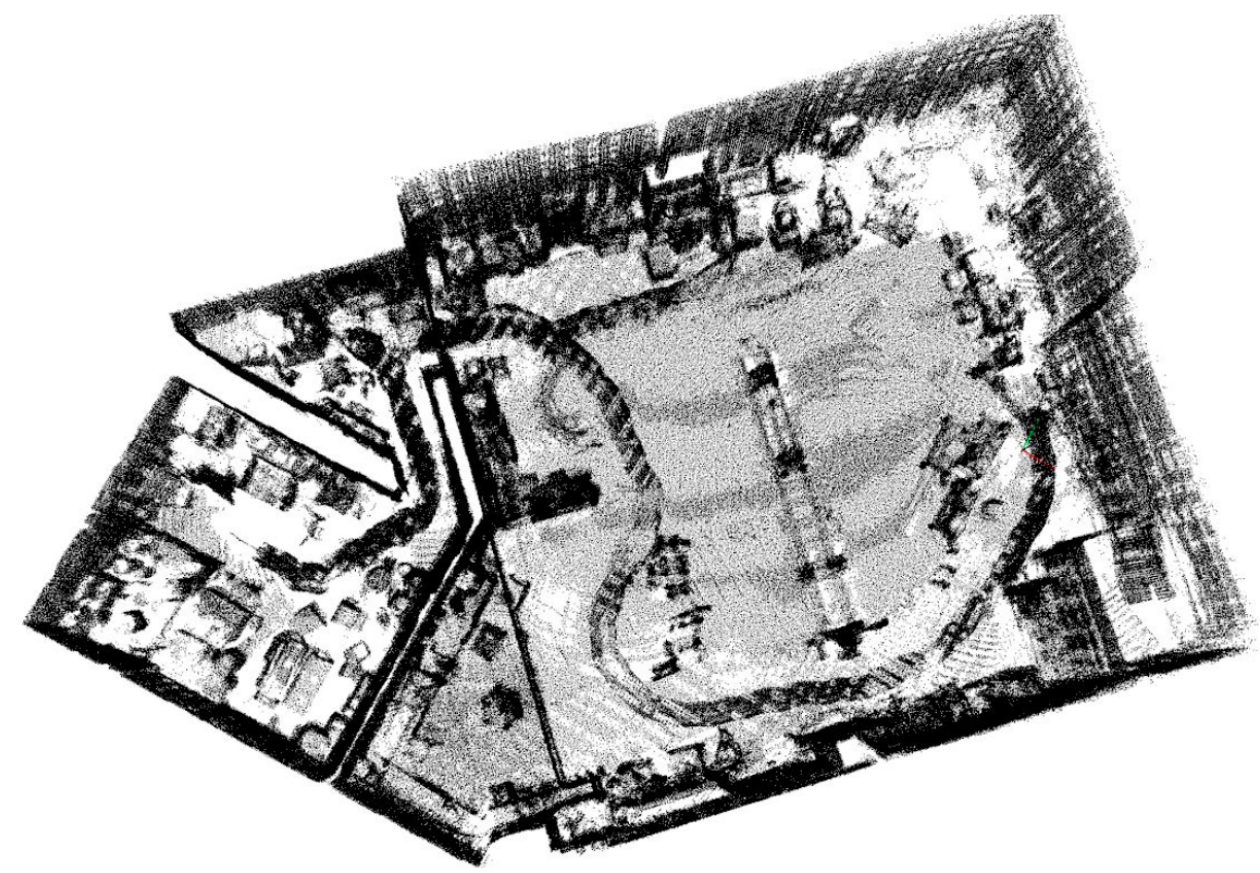

Figure 5.21: Point cloud of NRC laboratory generated by LIDAR scanner. Pointcloud image made by Iain Cornick, University of Waterloo, and is property of the $N R C$.

To form a global point-cloud coordinate system like that presented in figure 5.21, the point-cloud data acquired at each dwell point were co-registered using an iterative closest-point (ICP) method. ${ }^{73,74}$ To execute the ICP algorithm, a set of reference points from the point-cloud dataset at the initial position was defined. For each subsequent position, points in the point cloud were identified which corresponded to 
the reference points. Rigid-body transformations were then applied to minimize the distance between the pairs of points. The coordinates of the LIDAR unit, along with its orientation, were extracted from the global point-cloud coordinate system. The coordinates and orientation of the $3 \times 3$-module SCoTSS detector were then obtained by transformation from the LIDAR frame of reference to that of the detector, and are presented in table 5.3.

Table 5.3: Detector coordinates and orientations for the dwell-point positions during the experimental point-source measurement. Coordinates are presented in the frame of reference used in figure 5.1. The orientations are measured with respect to the $x$-axis.

\begin{tabular}{c|c|c|c} 
Dwell point & $\mathrm{x}(\mathrm{m})$ & $\mathrm{y}(\mathrm{m})$ & Detector orientation $(\mathrm{deg})$ \\
\hline 0 & $13.56 \pm 0.01$ & $8.85 \pm 0.01$ & 167.0 \\
1 & $11.54 \pm 0.01$ & $4.39 \pm 0.01$ & 137.8 \\
2 & $8.22 \pm 0.01$ & $0.47 \pm 0.01$ & 98.6 \\
3 & $5.12 \pm 0.01$ & $2.91 \pm 0.01$ & 63.4 \\
4 & $0.87 \pm 0.01$ & $6.75 \pm 0.01$ & 29.1 \\
5 & $2.24 \pm 0.01$ & $10.15 \pm 0.01$ & 334.9 \\
6 & $4.84 \pm 0.01$ & $13.12 \pm 0.01$ & 314.8 \\
7 & $6.16 \pm 0.01$ & $15.36 \pm 0.01$ & 296.8 \\
8 & $10.27 \pm 0.01$ & $14.67 \pm 0.01$ & 260.0
\end{tabular}

\subsubsection{Monte Carlo simulation of detector response}

\section{Simulation geometry}

The point-model response and the response templates generated for spatial unfolding, each described in subsections below, were simulated in identical synthetic environments. As per the description given in section 4.6, only air was included in the simulated environment. The simulated air had a density of $1.2048 \times 10^{-3} \mathrm{~g} / \mathrm{cm}^{3}$, consistent with dry air at a temperature of $20{ }^{\circ} \mathrm{C}$ and at a pressure of $101.3 \mathrm{kPa}$.

The simulated representation of the interior components of each detector plane remained the same as was presented in figure 4.9. The dimensions of the sensitive components are as presented in chapter 3. For the configuration used in measuring the experimental point source, the detector additionally had a carbon fibre enclosure with a plastic lid and five-sided coverings of carbon fibre on each detector plane. Figure 5.22 
shows the simulated representation of the detector with additional carbon fibre and polystyrene enclosure used for the work presented in this chapter. Figure 5.22a shows a view of the detector from the front, while figure $5.22 \mathrm{~b}$ shows a view of the detector from the rear; in each subfigure, an oblique clipping-plane has been used to provide a cross-sectional view of the detector. Yellow material represents carbon fibre, dark red material represents polystyrene, green material represents $\mathrm{CsI}(\mathrm{Tl})$ and magenta material represents aluminium.

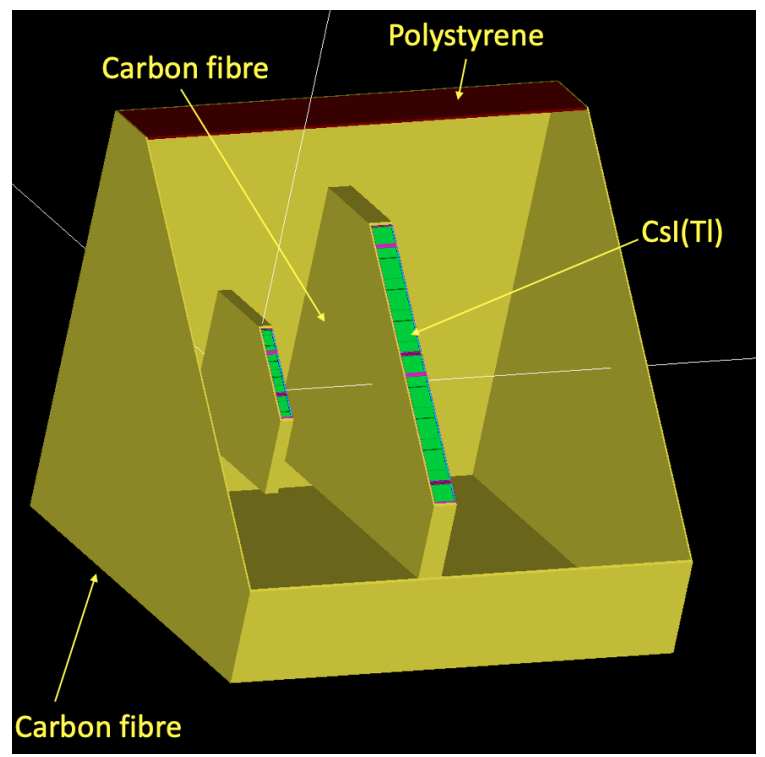

(a)

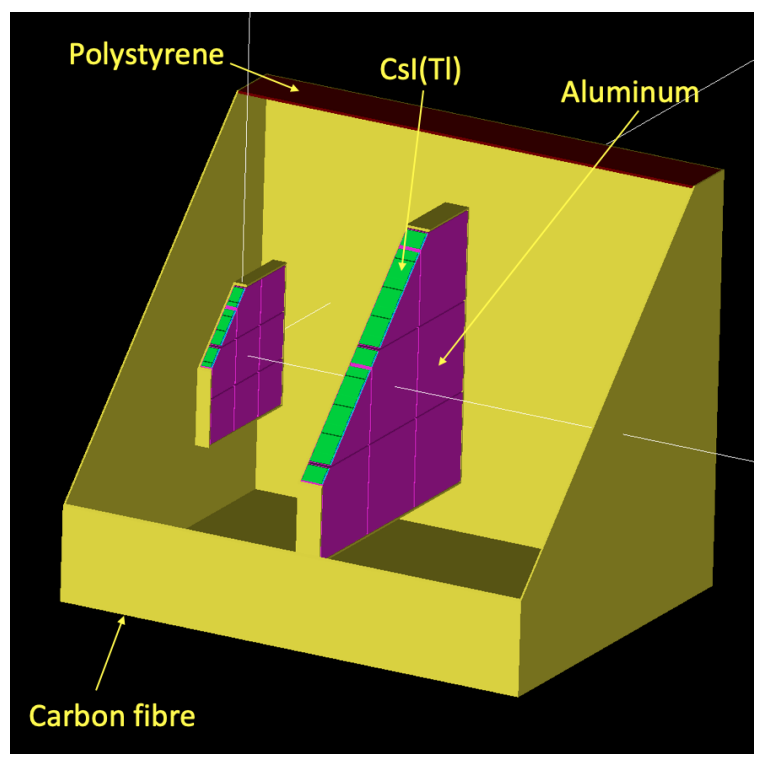

(b)

Figure 5.22: View of the simulated 3x3-module SCoTSS detector geometry used for the experimental point-source measurement. a) Visualization of the simulated detector from the front. b) Visualization of the simulated detector from the rear. The yellow material represents carbon fibre, the dark red material represents polystyrene, the green material represents CsI(Tl), and the magenta material represents aluminum. An oblique clipping-plane has been used in both subfigures to reveal a cross section through the interior of the detector.

To incorporate the resolution of each channel as it existed in the experimental configuration, the empirical energy-smearing function used in the MC simulations and provided in equation 4.9 was adjusted using calibration data measured at $662 \mathrm{keV}$ as per the procedure described in section 4.6. 


\section{Comparison of MC simulation to real data}

Figure 5.23 shows a comparison of experimental Compton imaging measurements to synthetic data generated from a point source, as measured from dwell point 0 and following the application of the energy selection criteria given in table 5.1. The $662 \mathrm{keV}$ emission rate of the point source was 14.4 million/s and was determined by the total emission rate in the image space recovered by the spatial-unfolding method, the results for which will be presented in section 5.2.3. Figures 5.23a and 5.23b show the distribution of Compton cone polar angles and Compton scattering angles, respectively, whereas figures 5.23c to 5.23e show the energy depositions in the scatter plane, in the absorber plane, and in the full detector for all two-hit events. Figures 5.24 to 5.31 show the same results for dwell points 1 to 8 . These figures show that the MC simulation does well in reproducing the structure of the experimental data. The agreement between MC and experimental data supports the use of a collimated source representation within the response simulations of the 3x3-module SCoTSS detector, as the physical processes revealed by the distributions of the experimental data are well reproduced by the $\mathrm{MC}$ simulation. 


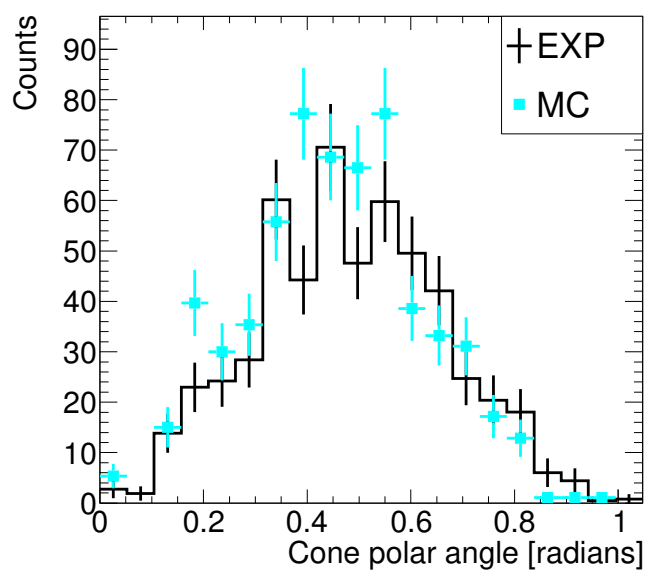

(a)

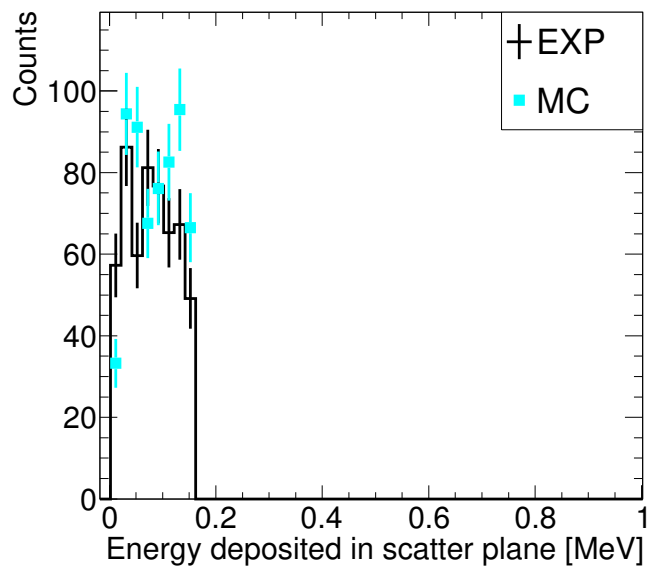

(c)

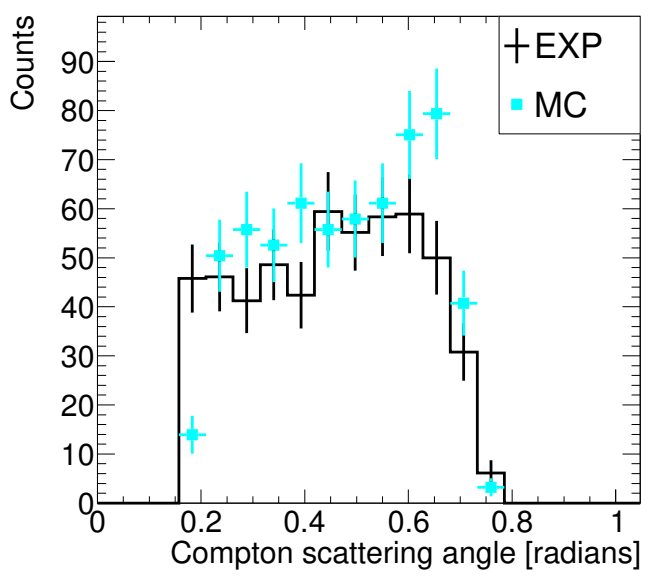

(b)

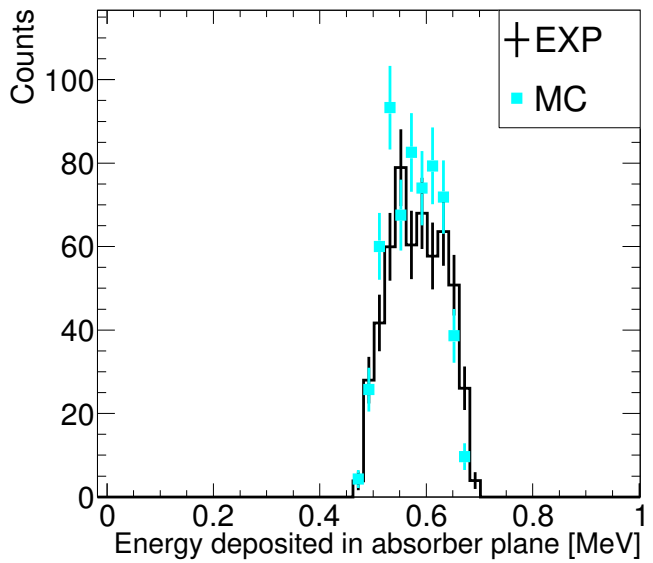

(d)

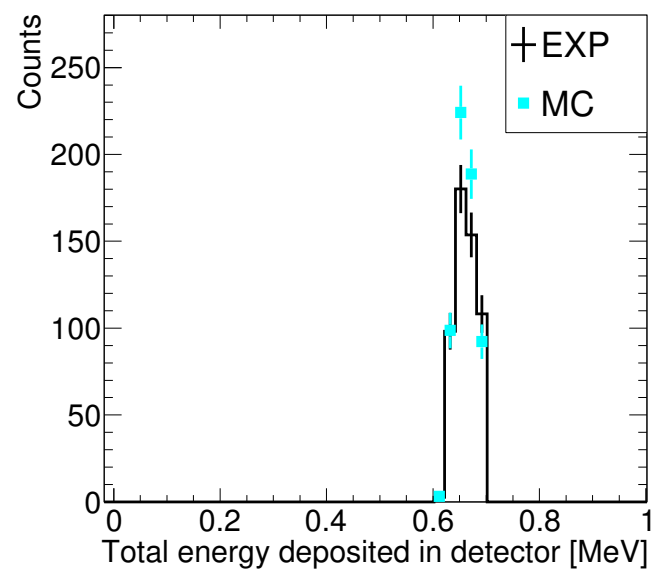

(e)

Figure 5.23: Simulated and experimental point-source Compton imaging measurements at dwell point 0 after energy cuts. a) Compton cone polar angle. b) Compton scattering angle. c) Scatter-plane energies. d) Absorber-plane energies. e) Totalenergy depositions. 


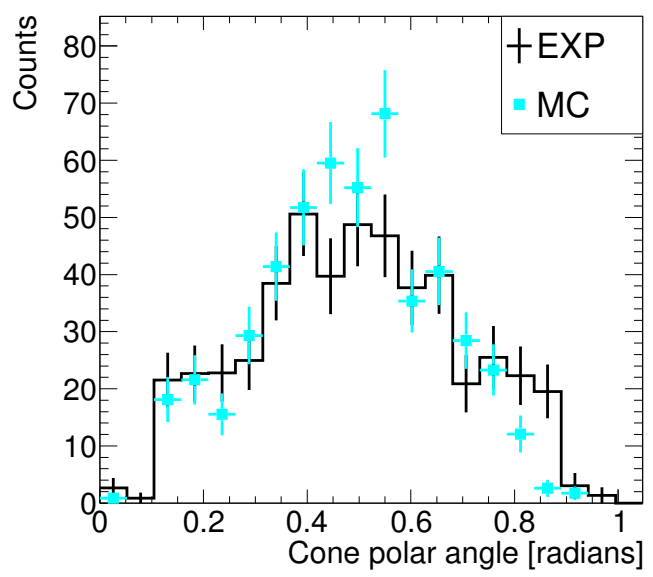

(a)

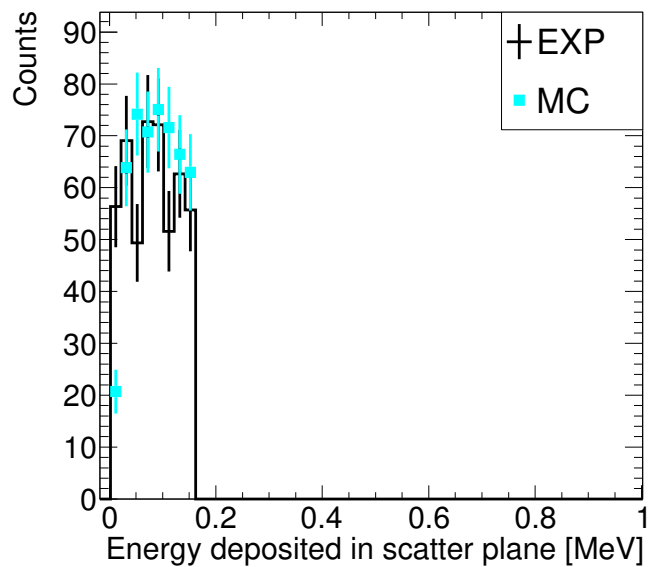

(c)

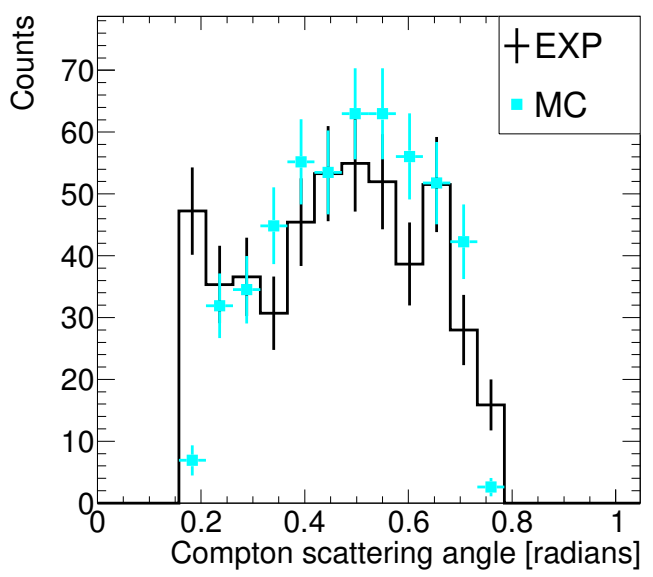

(b)

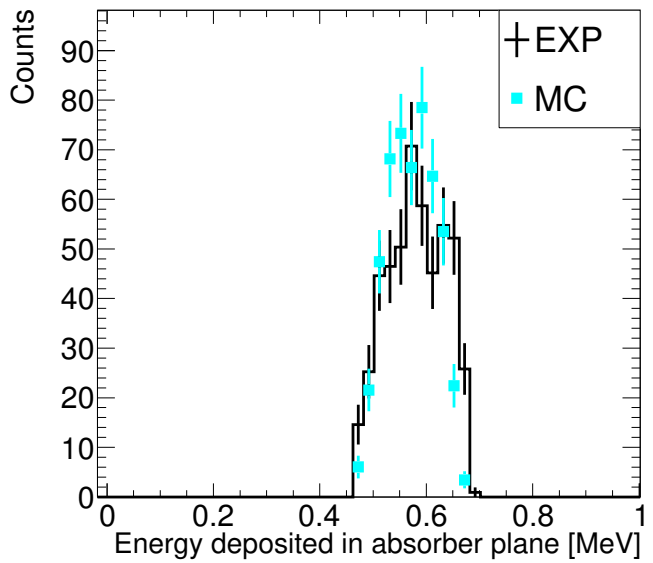

(d)

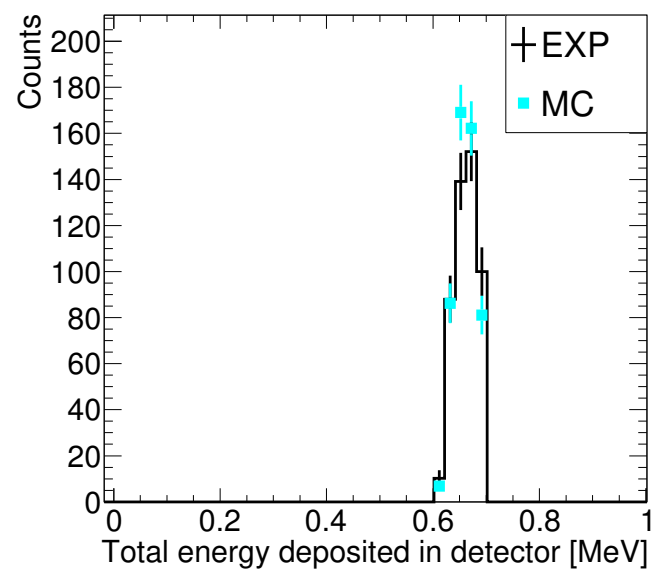

(e)

Figure 5.24: Simulated and experimental point-source Compton imaging measurements at dwell point 1 after energy cuts. a) Compton cone polar angle. b) Compton scattering angle. c) Scatter-plane energies. d) Absorber-plane energies. e) Totalenergy depositions. 


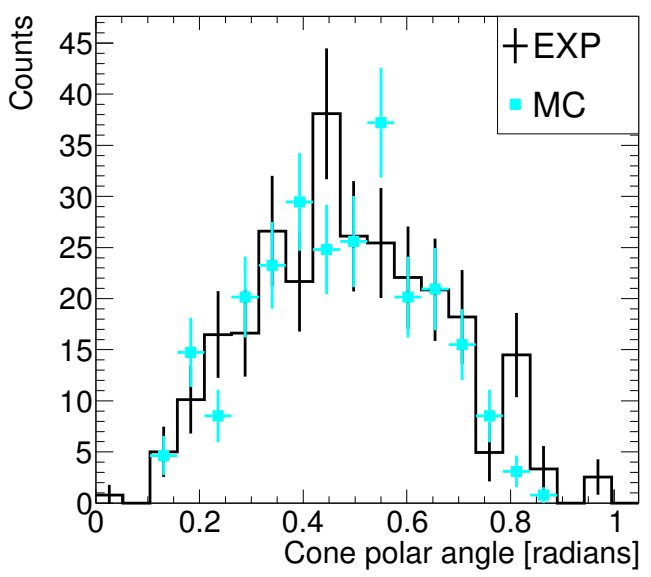

(a)

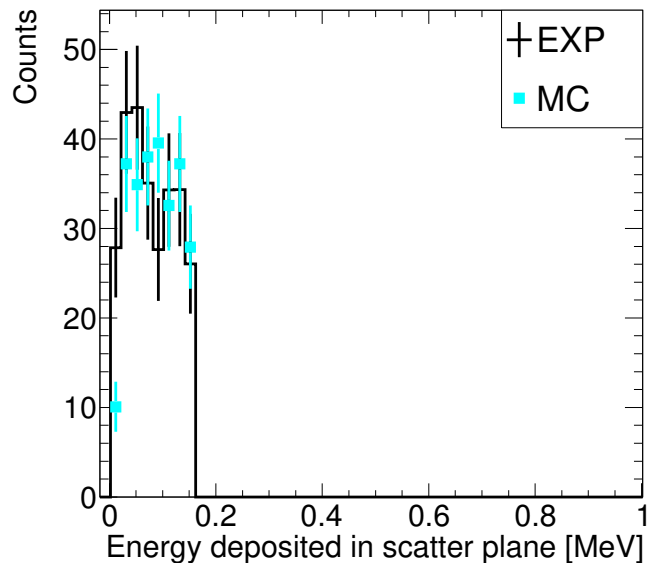

(c)

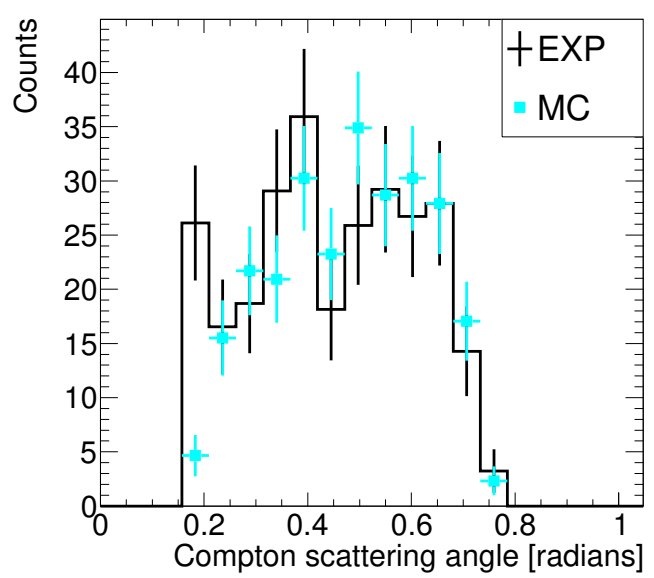

(b)

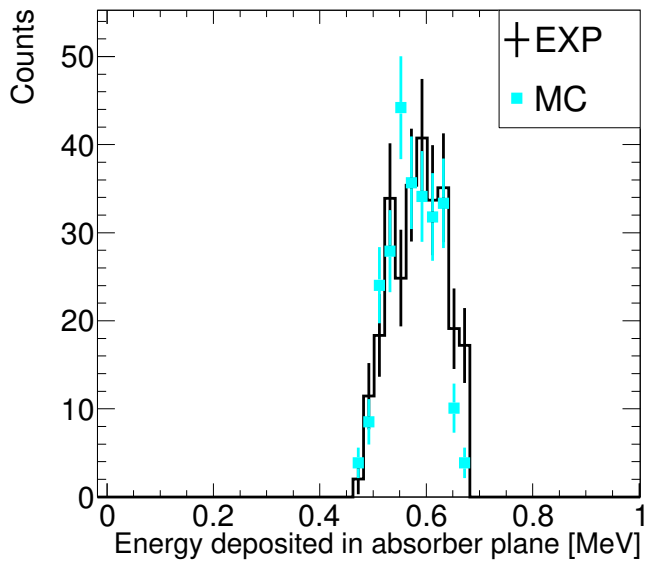

(d)

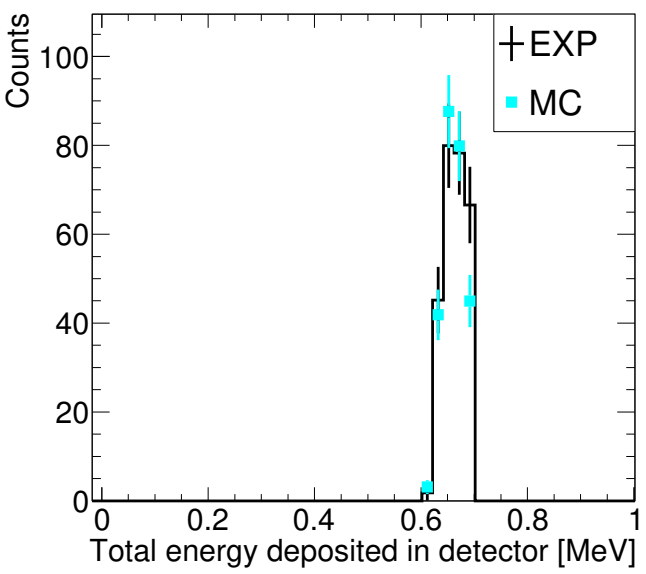

(e)

Figure 5.25: Simulated and experimental point-source Compton imaging measurements at dwell point 2 after energy cuts. a) Compton cone polar angle. b) Compton scattering angle. c) Scatter-plane energies. d) Absorber-plane energies. e) Totalenergy depositions. 


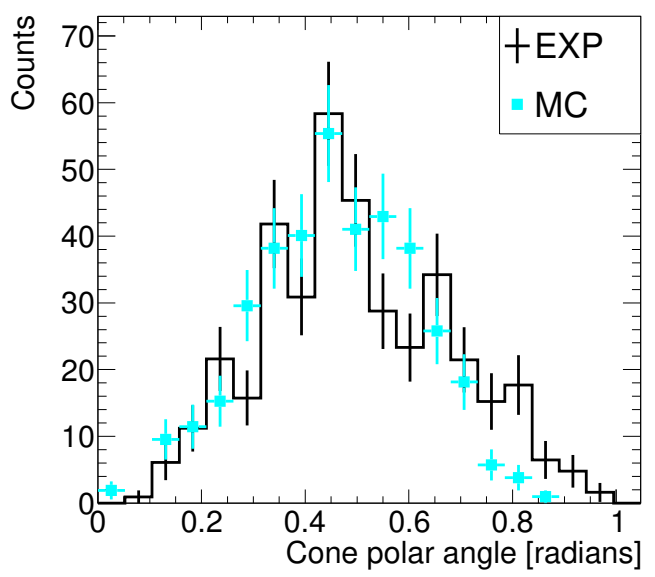

(a)

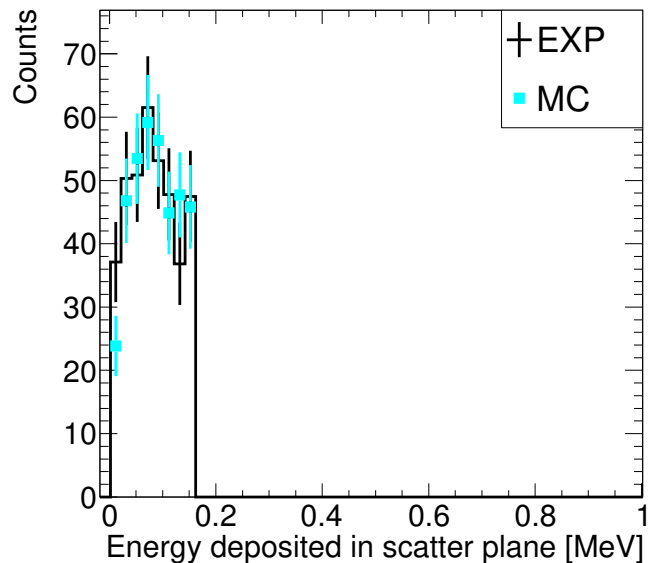

(c)

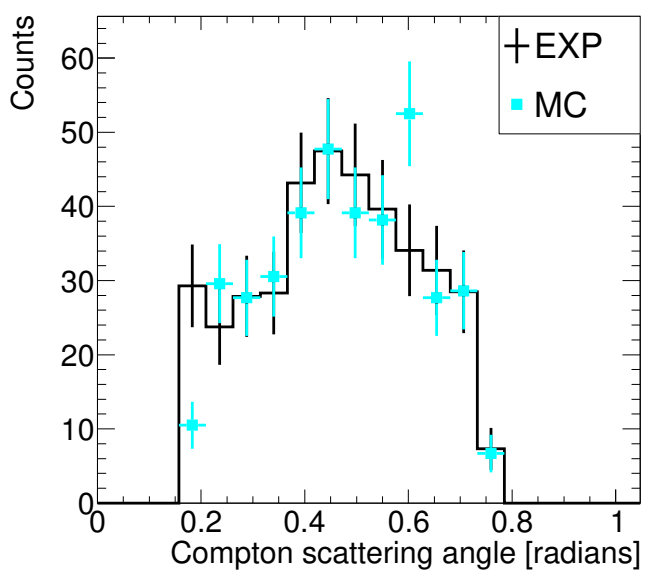

(b)

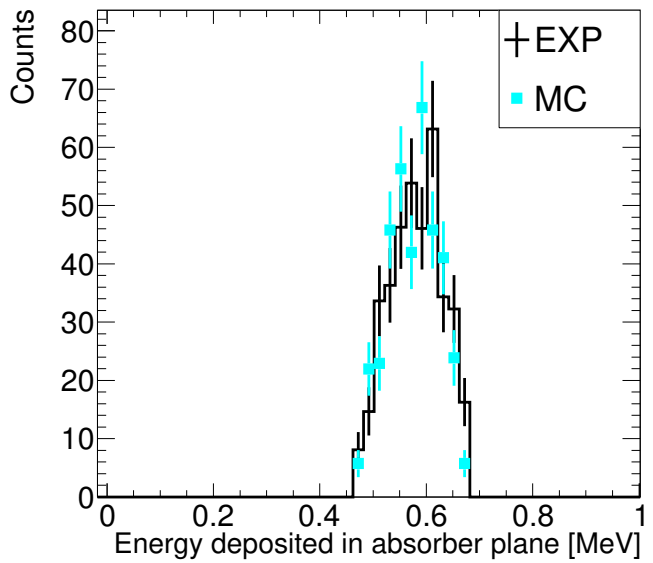

(d)

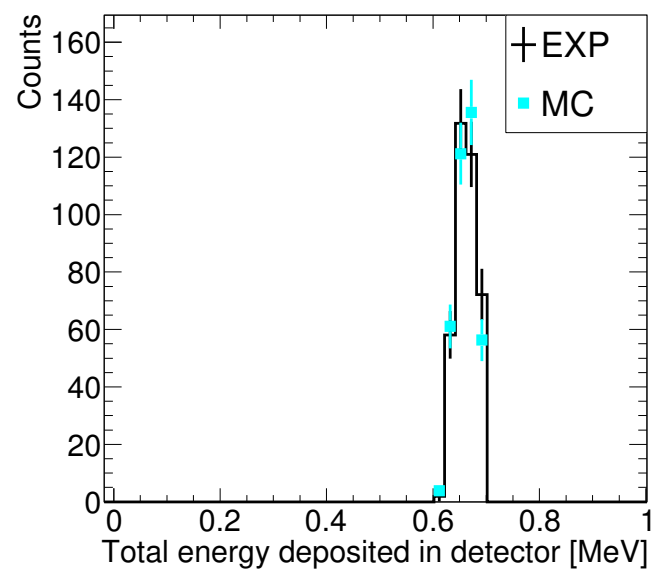

(e)

Figure 5.26: Simulated and experimental point-source Compton imaging measurements at dwell point 3 after energy cuts. a) Compton cone polar angle. b) Compton scattering angle. c) Scatter-plane energies. d) Absorber-plane energies. e) Totalenergy depositions. 


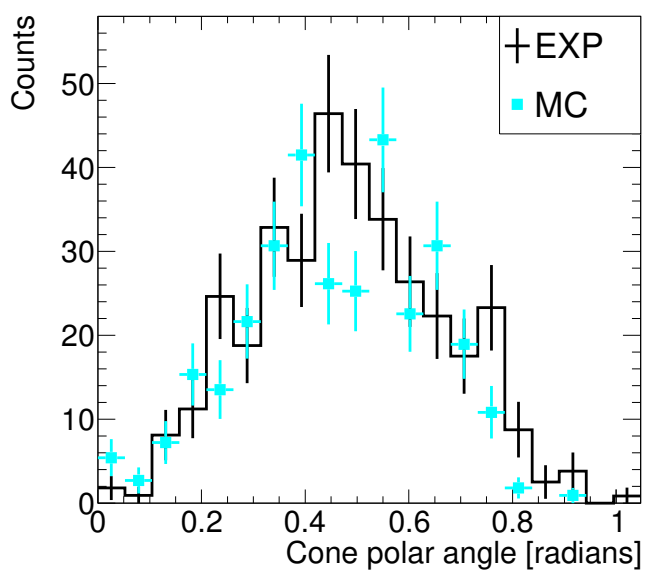

(a)

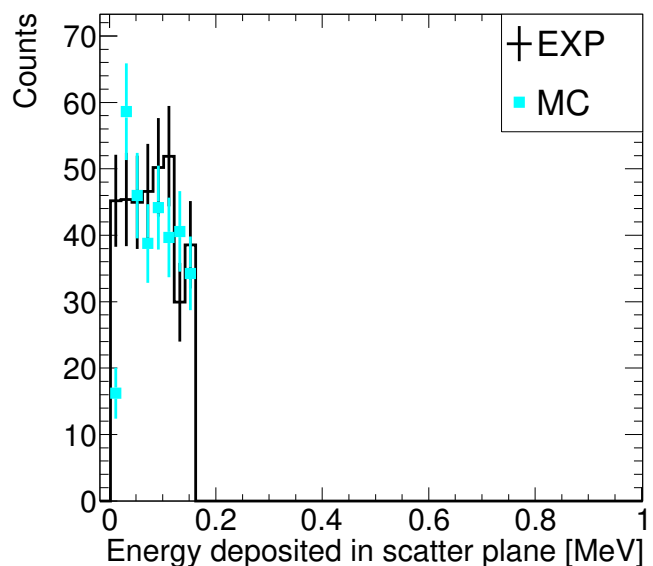

(c)

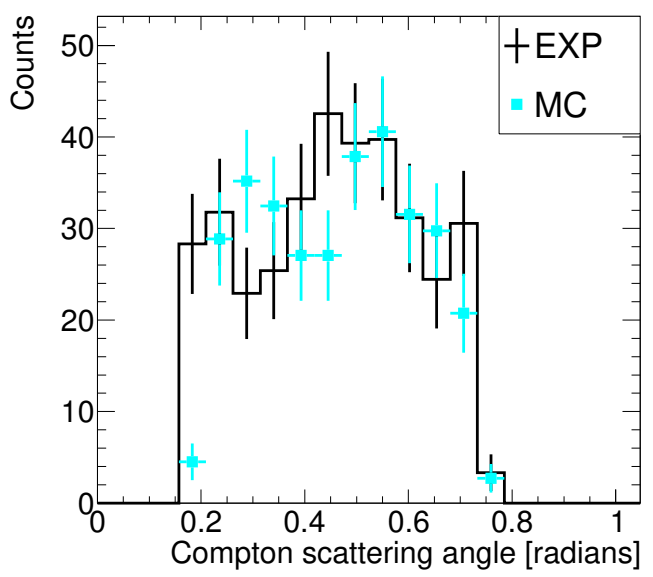

(b)

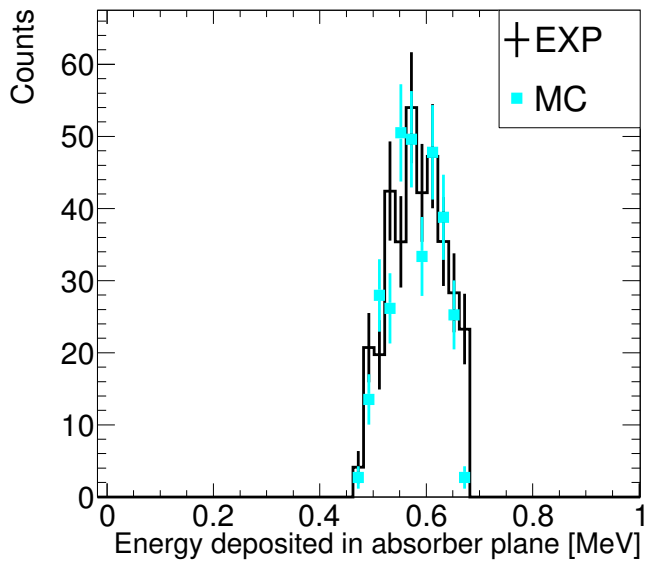

(d)

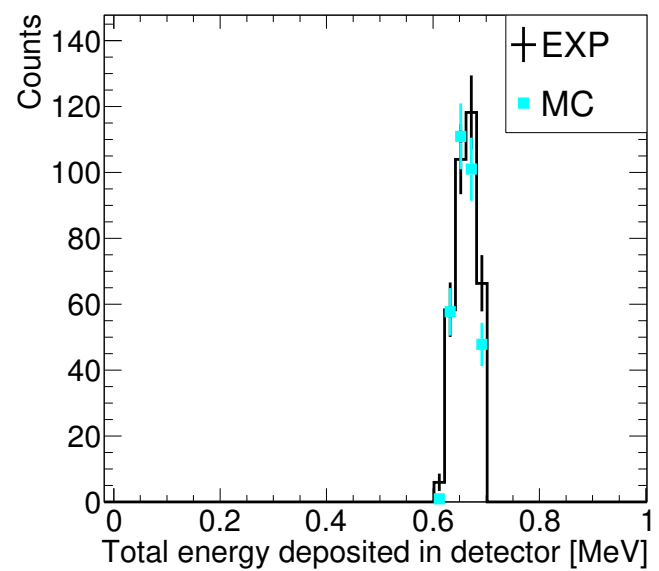

(e)

Figure 5.27: Simulated and experimental point-source Compton imaging measurements at dwell point 4 after energy cuts. a) Compton cone polar angle. b) Compton scattering angle. c) Scatter-plane energies. d) Absorber-plane energies. e) Totalenergy depositions. 


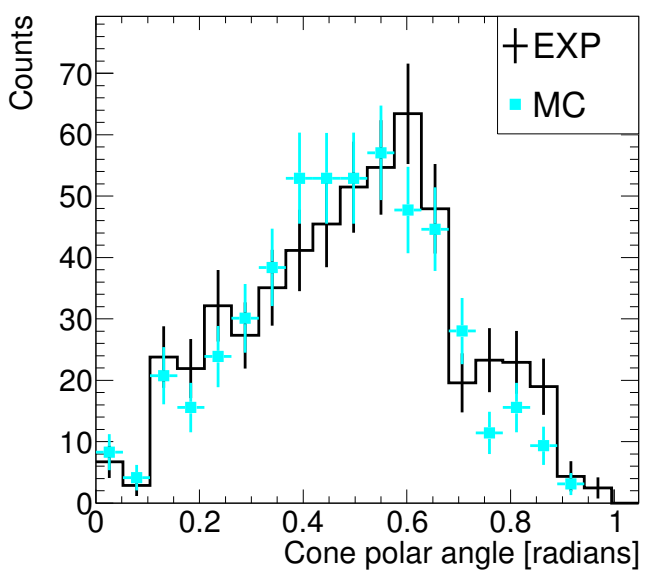

(a)

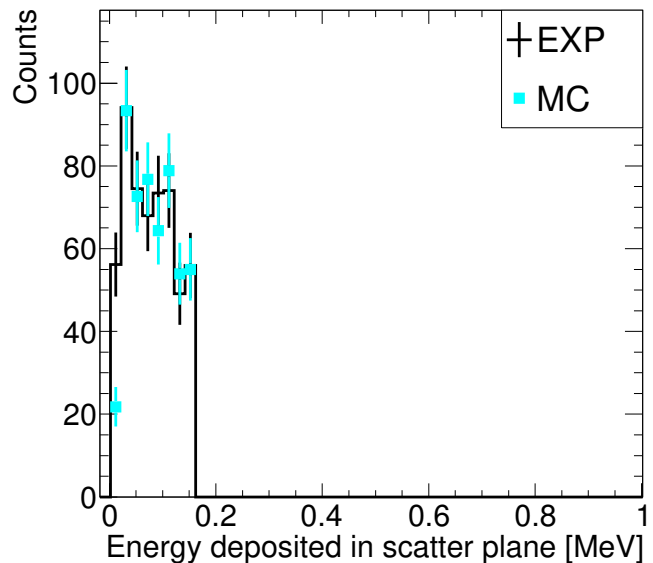

(c)

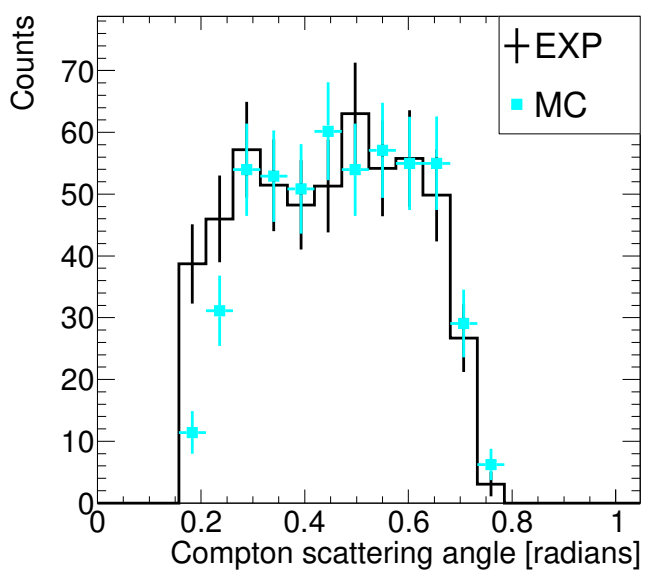

(b)

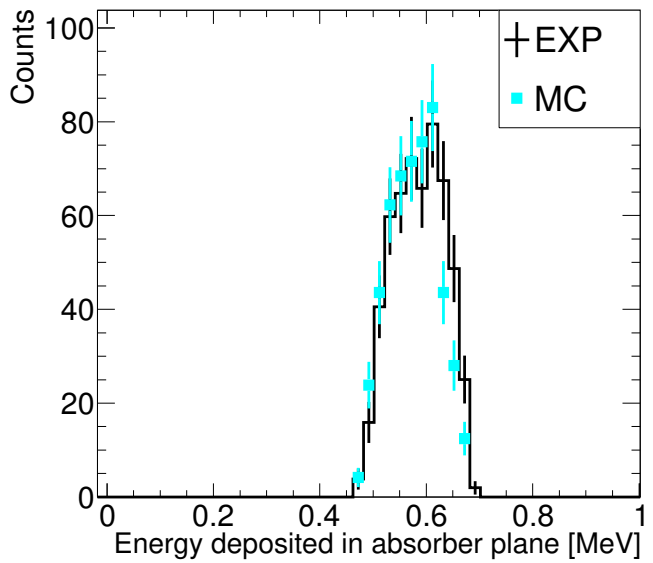

(d)

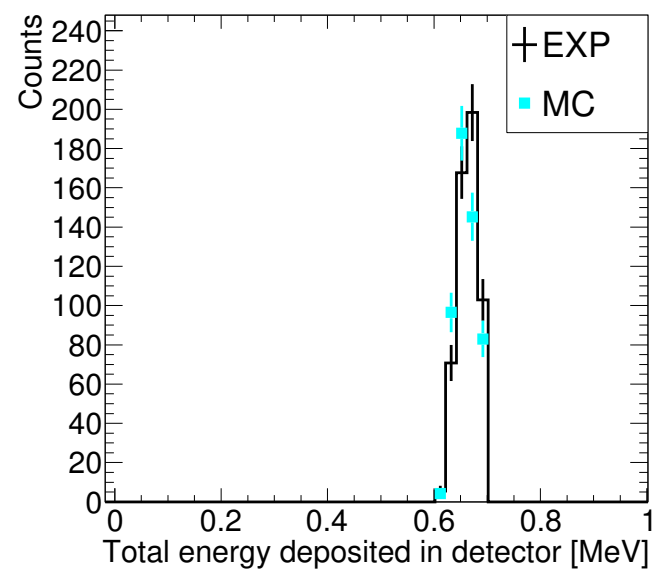

(e)

Figure 5.28: Simulated and experimental point-source Compton imaging measurements at dwell point 5 after energy cuts. a) Compton cone polar angle. b) Compton scattering angle. c) Scatter-plane energies. d) Absorber-plane energies. e) Totalenergy depositions. 


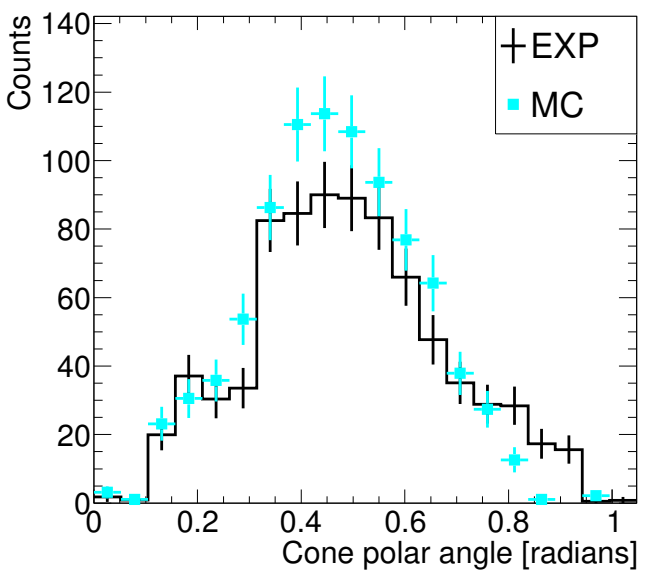

(a)

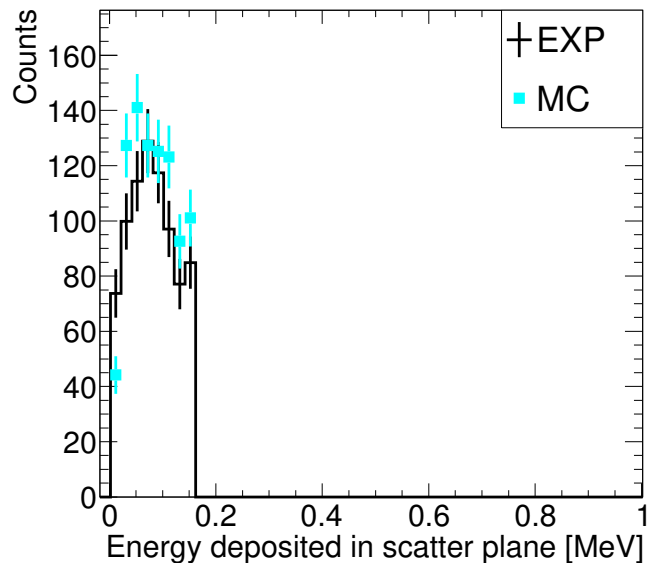

(c)

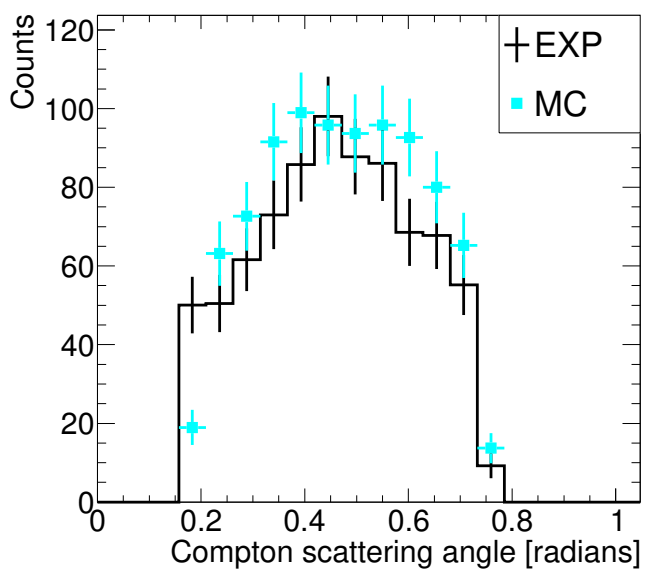

(b)

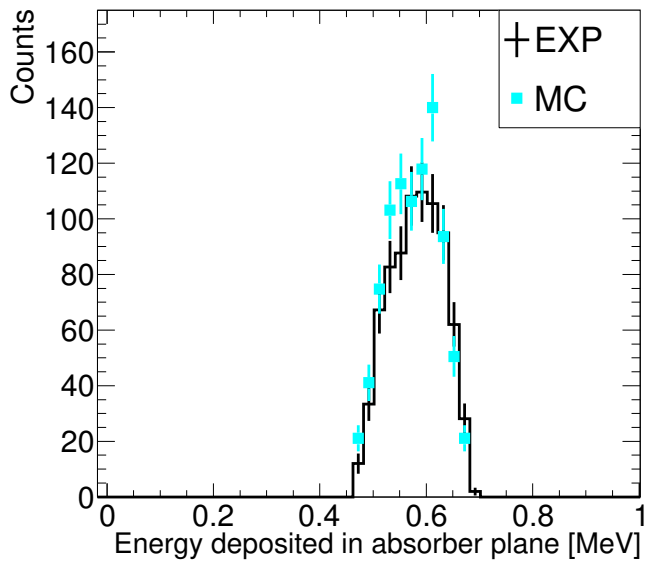

(d)

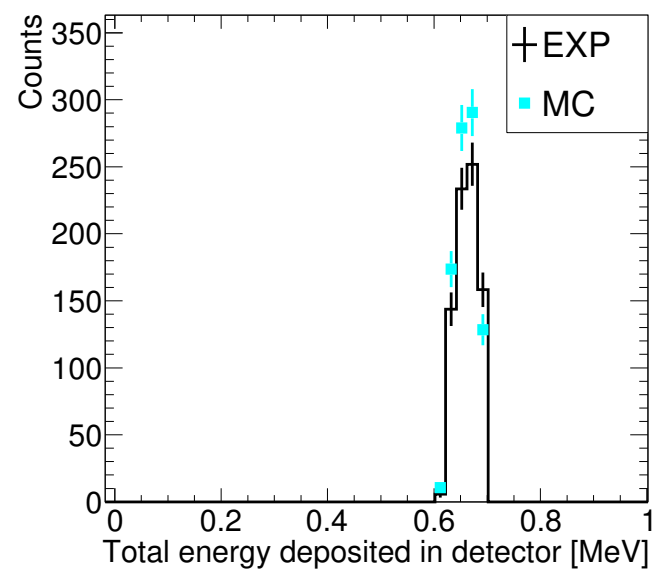

(e)

Figure 5.29: Simulated and experimental point-source Compton imaging measurements at dwell point 6 after energy cuts. a) Compton cone polar angle. b) Compton scattering angle. c) Scatter-plane energies. d) Absorber-plane energies. e) Totalenergy depositions. 
113

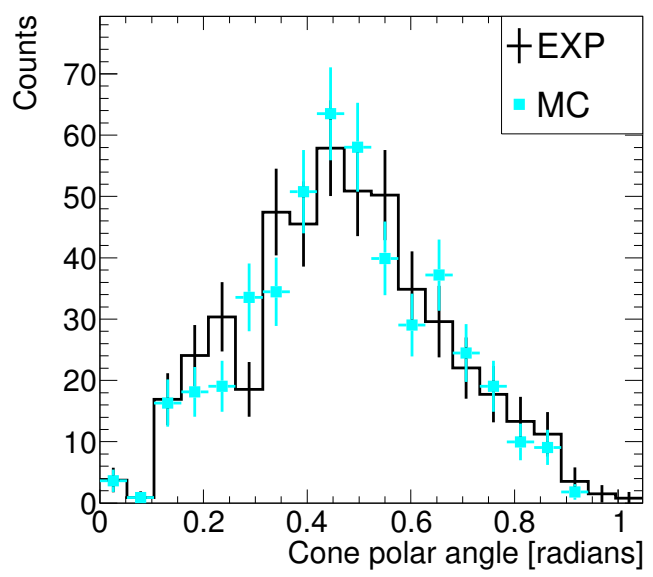

(a)

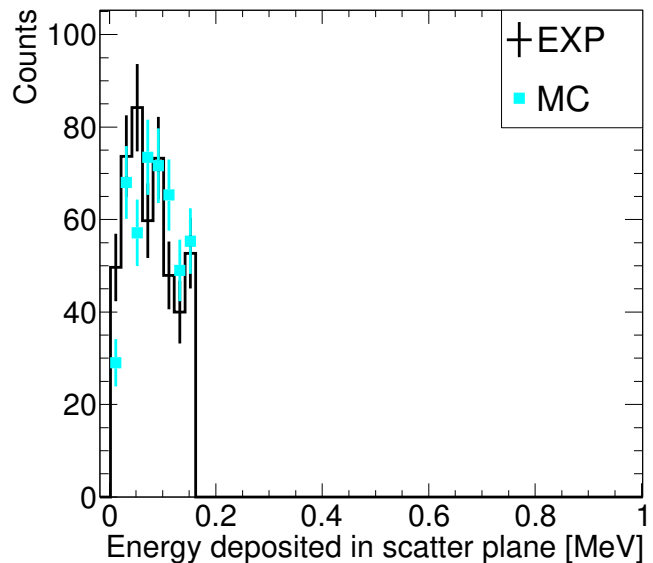

(c)

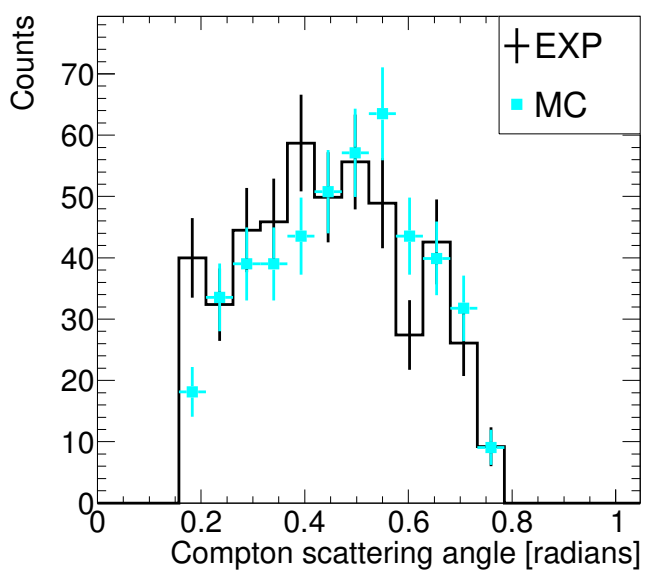

(b)

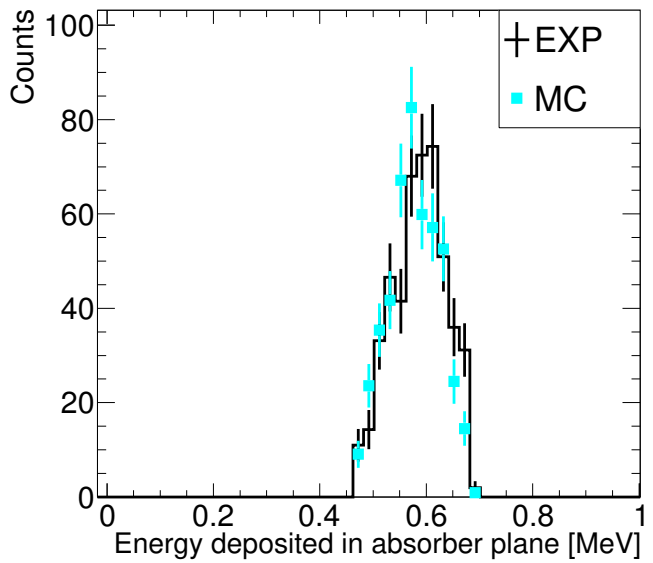

(d)

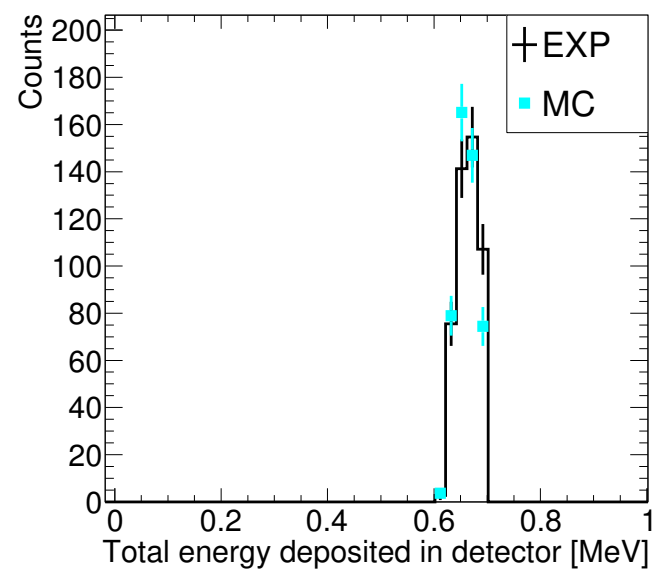

(e)

Figure 5.30: Simulated and experimental point-source Compton imaging measurements at dwell point 7 after energy cuts. a) Compton cone polar angle. b) Compton scattering angle. c) Scatter-plane energies. d) Absorber-plane energies. e) Totalenergy depositions. 


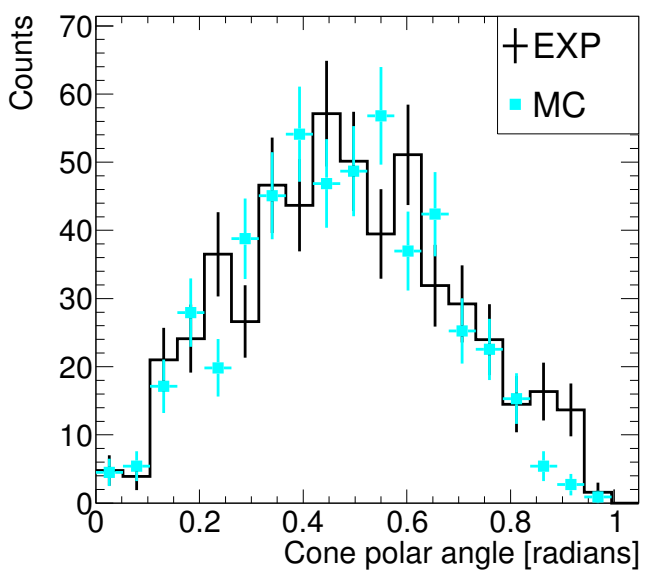

(a)

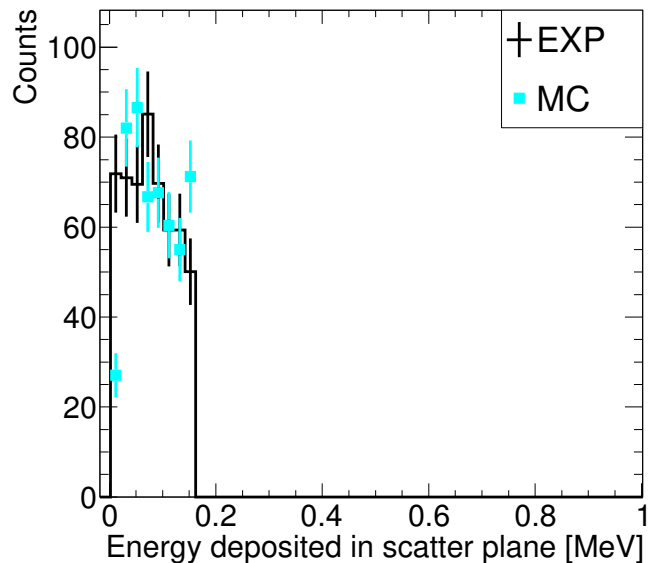

(c)

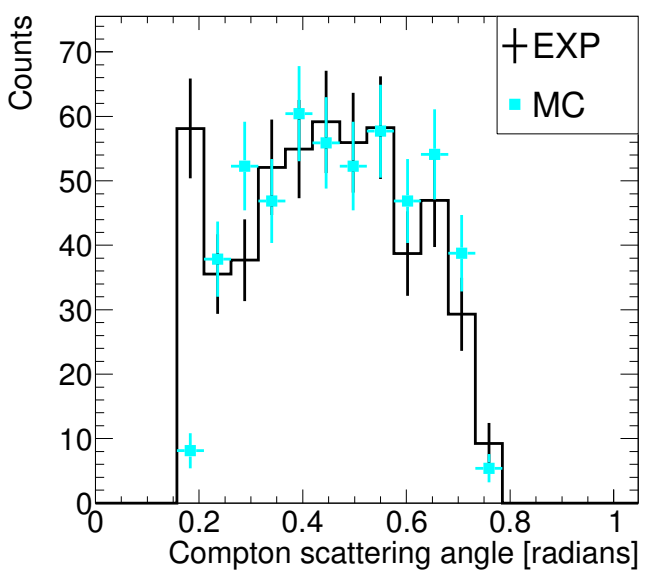

(b)

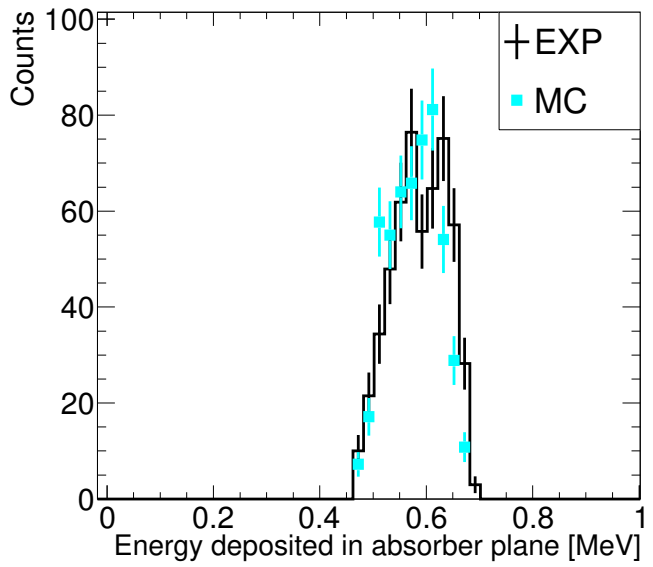

(d)

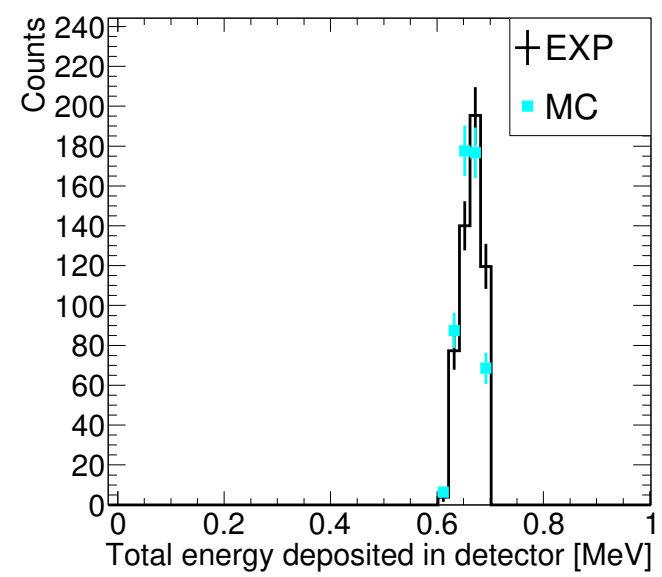

(e)

Figure 5.31: Simulated and experimental point-source Compton imaging measurements at dwell point 8 after energy cuts. a) Compton cone polar angle. b) Compton scattering angle. c) Scatter-plane energies. d) Absorber-plane energies. e) Totalenergy depositions. 


\section{Point-model response}

The point-model estimate that is applied to obtain a rapid estimate of the source strength requires that the two-hit event rate of the detector be parametrized. The response of the 3x3-module SCoTSS detector to a monoenergetic point-source of $662 \mathrm{keV}$ photons was parametrized by simulating point sources at increasing distance away from the detector, ranging from $3 \mathrm{~m}$ to $10 \mathrm{~m}$. The angular dependence of the response was parametrized by simulating point sources at increasing angle from the central axis of the detector up to $80^{\circ}$ and at a fixed distance of $5 \mathrm{~m}$. The simulated datasets used 250 million collimated histories per point-source simulation. In each simulation, the point source was placed $15.4 \mathrm{~cm}$ above the central plane of the detector as per the experimental configuration. Each simulation took a combined computation time of approximately 6 hours over 10 Intel Xeon E5620 processors.

The simulated data were then passed through the selection criteria presented in table 5.1. The distance-dependent response was parametrized by normalizing the number of events accepted by the total number of simulated decays. The angular response correction was obtained by normalizing the number of events accepted from simulations of a point source at increasing angle by the value on the central axis. Figure 5.32 shows the parametrizations obtained for the response of the 3x3-module SCoTSS detector to a monoenergetic point-source of $662 \mathrm{keV}$ photons that will be used in the point-model fit method for estimating the source activity rapidly. 


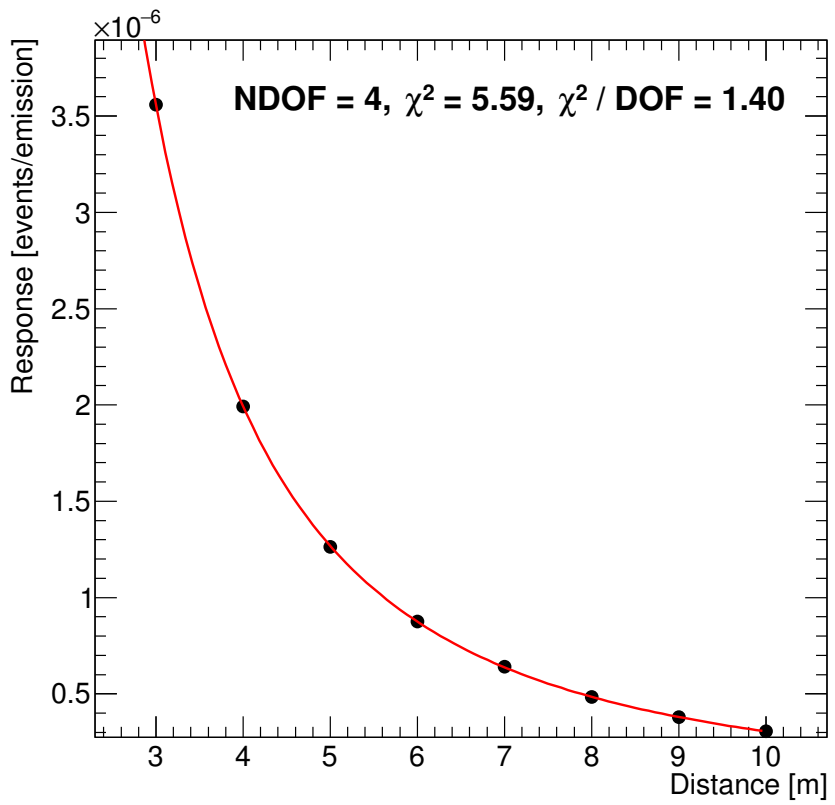

(a)

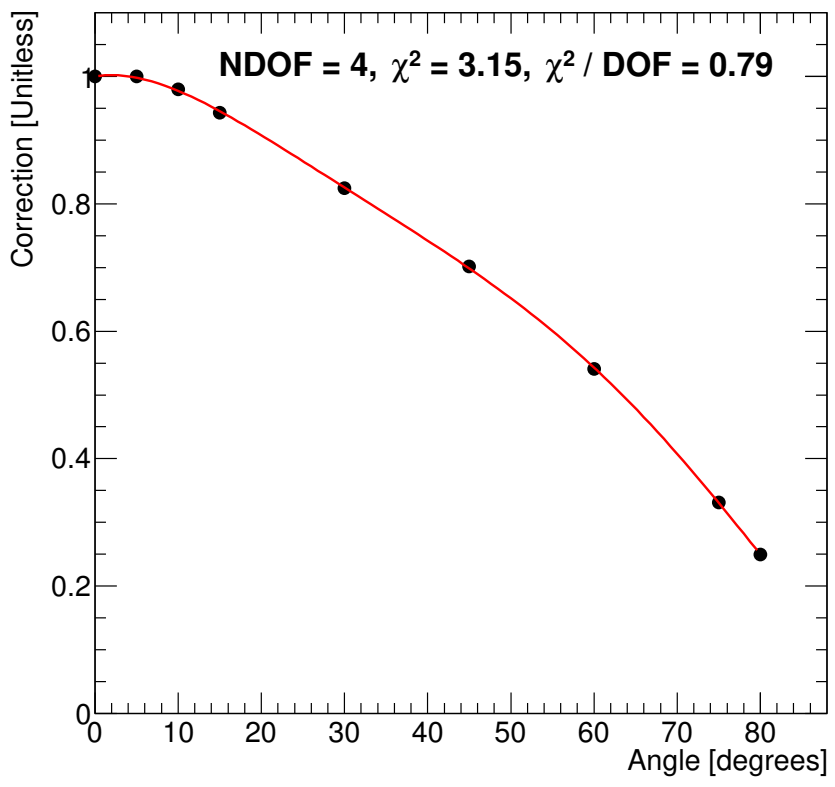

(b)

Figure 5.32: Point-response parametrization of a monoenergetic 662 keV point source. a) Response of the detector to an on-axis point source as a function of distance, $f_{\text {dist }}(r)$. b) Correction for angular response, $f_{\text {ang }}\left(\theta_{\text {axis }}\right)$. In both subfigures, the uncertainty bars on the data points are too small to be visualized. 


\section{Verification of tomographic reconstruction using MC simulation}

The tomographic reconstruction methodology described in section 4.5 was utilized to produce tomographic results from a synthetic point source simulated in the experimental geometry with a $662 \mathrm{keV}$ photon emission rate of $17.4 \mathrm{million} / \mathrm{s}$ and containing similar statistics to that of the experimental data. Figure 5.33a shows the tomographic reconstruction of the full image space, where each of the dwell points has been marked by a red diamond and the true source location has been marked by a black cross. An additional quality cut has been enforced which zeroes pixels outside of the combined field of view of all nine dwell points. This quality cut represents an assumption that the dwell points enclose the source. Figure 5.33b shows a zoomed-in view of figure $5.33 \mathrm{a}$ in the vicinity of the source. The tomographic reconstruction demonstrates a region of high intensity at the known location of the source. The tomographic reconstruction also demonstrates "spoking" properties which extend from each of the dwell points towards the region of maximum intensity. These "spokes" are known artefacts in tomographic back-projection methodologies ${ }^{27,75}$ and the spatialunfolding method presented later in this chapter will remove these artefacts. 


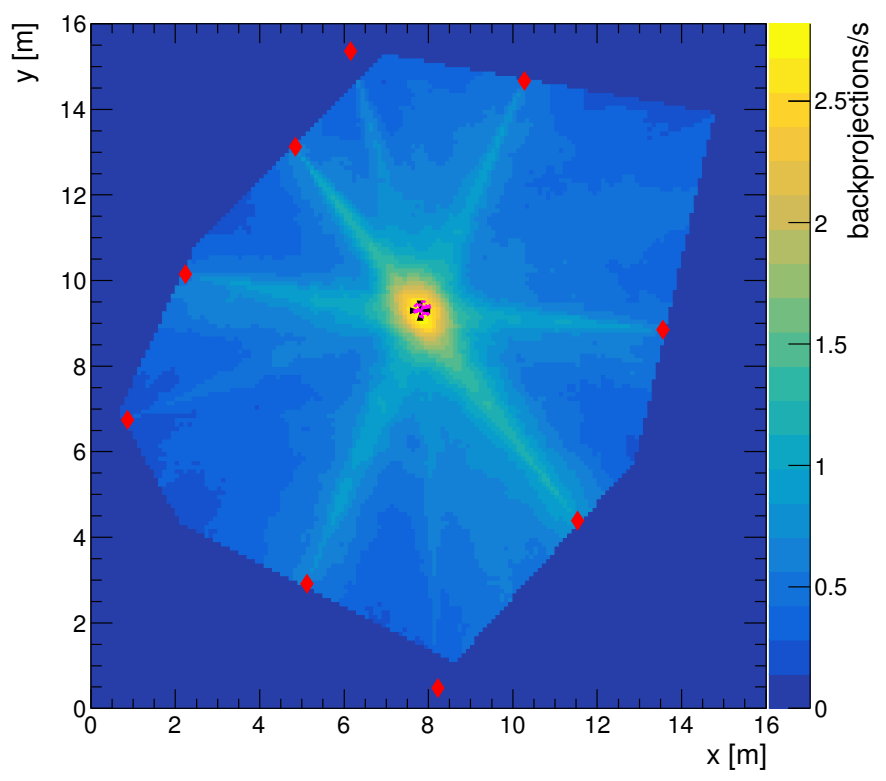

(a)

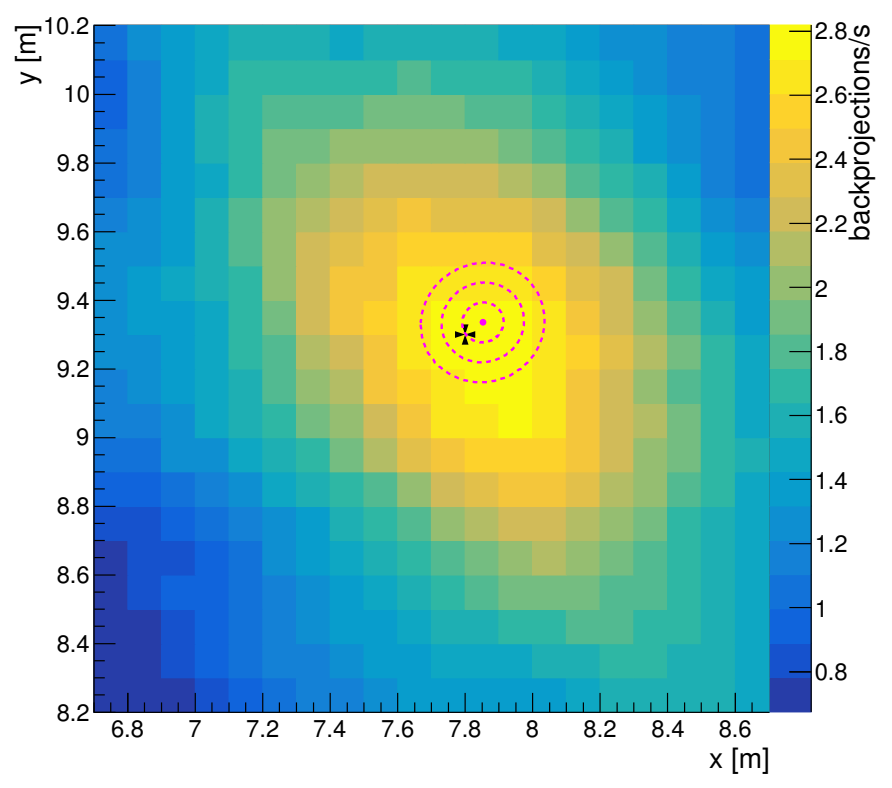

(b)

Figure 5.33: Tomographic reconstruction of synthetic monoenergetic 662 ke V point source with point-response approximation overlaid. a) Full image space. b) Zoomed-in view at location of source. Red diamonds mark the dwell points, the point-model fit result has been overlaid with a magenta dot, and magenta dashed lines represent the $1 \sigma, 2 \sigma$, and $3 \sigma$ spatial-uncertainty contours. A black cross marks the true location of the point source. The temporal rate arises from normalization by measurement time at each dwell point. 


\section{Verification of point-model response method using MC simulation}

Overlaid on the tomographic reconstructions in figure 5.33 is the point-model fit, where the placement of the source is marked by a magenta dot and dashed magenta lines represent the $1 \sigma, 2 \sigma$, and $3 \sigma$ spatial contours. The fit converged with a $\chi^{2}$ value of 4.8 for 6 degrees of freedom. The point-model fit had coordinates of $x=$ $(7.85 \pm 0.06) \mathrm{m}$ and $y=(9.34 \pm 0.06) \mathrm{m}$ with a $662 \mathrm{keV}$ photon emission rate of $(17.5 \pm 0.3)$ million/s. The reconstructed emission rate is therefore in agreement with the generated value of 17.4 million/s, and the source placement agrees with the expected location of the source. This synthetic result demonstrates that the pointmodel fit method works when the simulated response of the detector includes all effects present in the measurement.

\section{Response templates}

For the application of the spatial-unfolding method, the response of the $3 \times 3$-module SCoTSS detector to spatially-localized sources of $662 \mathrm{keV}$ gamma rays is required. The template response of the 3x3-module SCoTSS detector to spatially-localized sources of monoenergetic $662 \mathrm{keV}$ photons was obtained by simulating a $16 \mathrm{~m}$ by $16 \mathrm{~m}$ uniform sheet source placed $15.4 \mathrm{~cm}$ above the central plane of the detector, in the appropriate orientation relative to each of the nine dwell points in order to reproduce the measurement geometry of the experimental point-source measurement. A sheet source was simulated because this allows for calculation of the detector's response to the full image space using a single simulation; the emission positions of collimated histories from the sheet source in MC allows for the templates $f_{i}$ to be generated by dividing the sheet source into tiles of arbitrary size in post-processing. One simulation was performed per dwell point. Each simulation contained 5.12 billion collimated histories and required a combined total computation time of approximately 145 hours spread over 250 Intel Xeon E5620 processors.

The simulated data from the sheet source were divided into $1 \mathrm{~m}$ by $1 \mathrm{~m}$ tiles using the known emission location of each primary photon, providing 256 spatiallylocalized sources which correspond to the $f_{i}$ of section 4.8. This sorting process took approximately 6 hours to complete on a single $3.1 \mathrm{GHz}$ Intel Core i5 processor. For each $f_{i}$, the simulated photons that reached the detector, deposited energy in one 
crystal in each plane of the detector, and that passed the selection criteria provided in table 5.1 were then used in reconstructing the tomographic response templates $g_{i}$ by passing the data through the reconstruction chain described in section 4.5 followed by normalization of the response template by the simulated number of decays. This last step took approximately 4 hours to complete on a single $3.1 \mathrm{GHz}$ Intel Core i5 processor.

\subsection{Results and discussion}

\subsubsection{Uncorrected tomographic reconstruction}

The tomographic reconstruction methodology presented in section 4.5 were utilized for reconstruction of the experimentally-acquired point-source data, and a quality cut was enforced that zeroed pixels outside of the combined field of view of all dwell points. Because the location of the point source was known and the source itself was spatially compact, the back-projection of the Compton ring data at each dwell point was performed at the vertical angle in the field of view at which the point source sat. Table 5.4 lists the vertical angles used for tomographic back-projection at each dwell point.

Table 5.4: Vertical back-projection angles used in the experimental point-source reconstruction.

\begin{tabular}{c|c} 
Dwell point & Vertical angle used for back-projection (deg) \\
\hline 0 & 1.53 \\
1 & 1.43 \\
2 & 1.00 \\
3 & 1.27 \\
4 & 1.19 \\
5 & 1.57 \\
6 & 1.82 \\
7 & 1.40 \\
8 & 1.49
\end{tabular}

Figure 5.34 shows the ARM distribution obtained for a synthetic monoenergetic point source emitting $662 \mathrm{keV}$ photons at a distance of $5 \mathrm{~m}$ from the detector, generated using the MC representation described in section 5.1.3. The ARM distribution 
had a standard deviation of $3.58^{\circ}$. A back-projected ray width of $3.6^{\circ}$ was therefore chosen for tomographic reconstruction.

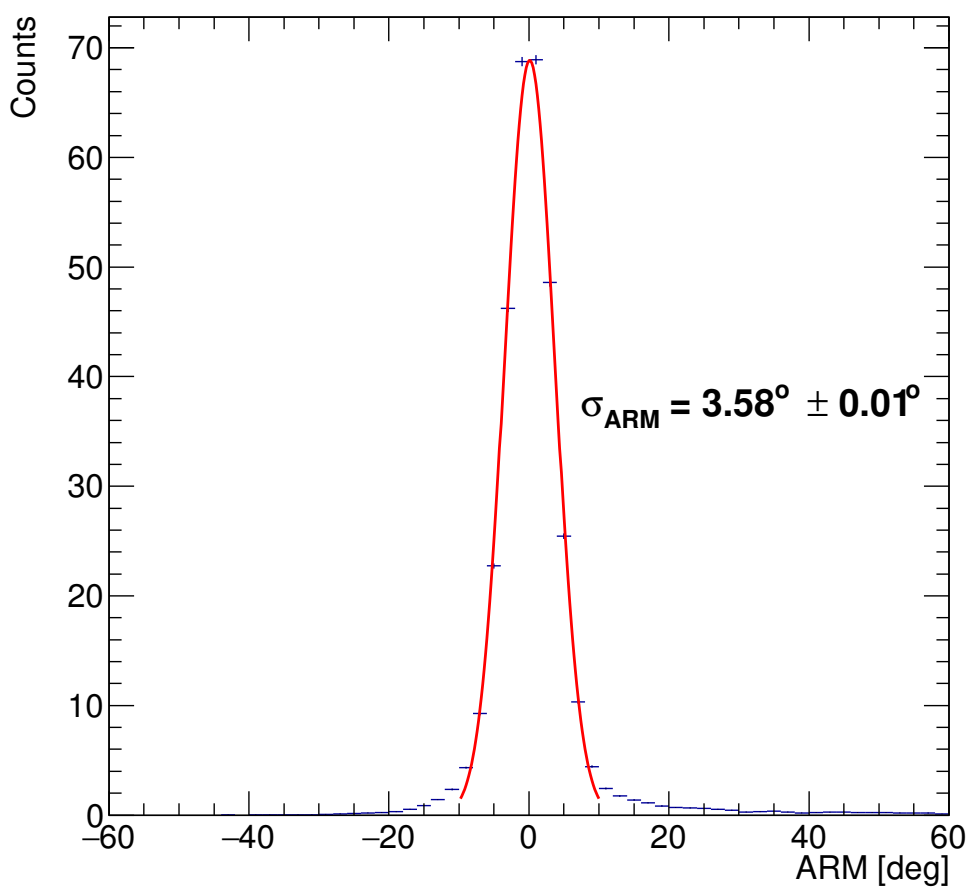

Figure 5.34: ARM distribution for a synthetic monoenergetic 662 keV point source.

Figure 5.35a shows a background-subtracted tomographic reconstruction of the experimental point-source measurements, where each of the dwell points has been marked by a red diamond and the true source location has been marked by a black cross. Figure 5.35b shows a zoomed-in view of figure 5.35a in the vicinity of the source. The tomographic reconstruction places a region of high intensity at the known source location. The tomographic reconstruction of the data took approximately 10 minutes on a laptop computer with a $3.1 \mathrm{GHz}$ Intel Core i5 processor, demonstrating the ability of the method to localize a gamma-ray source rapidly.

The tomographic reconstruction demonstrates "spoking" properties which extend from each of the dwell points towards the region of maximum intensity. These "spokes" are consistent with the patterns observed in the synthetic result shown in figure 5.33 and converge at a single relatively localized location, supporting the conjecture that the source is a point source. 


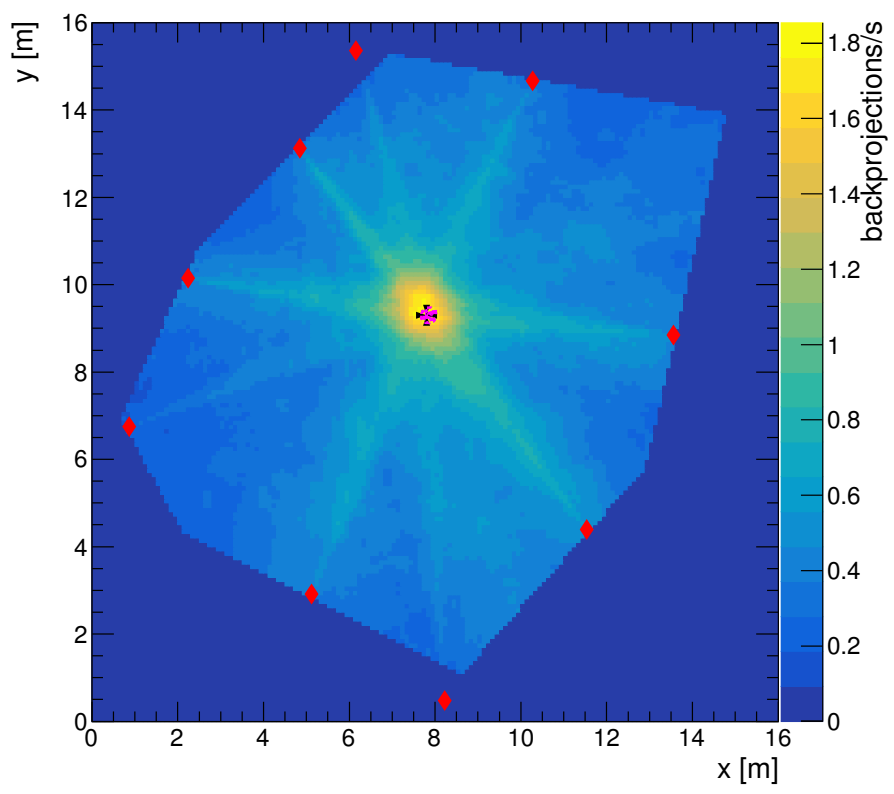

(a)

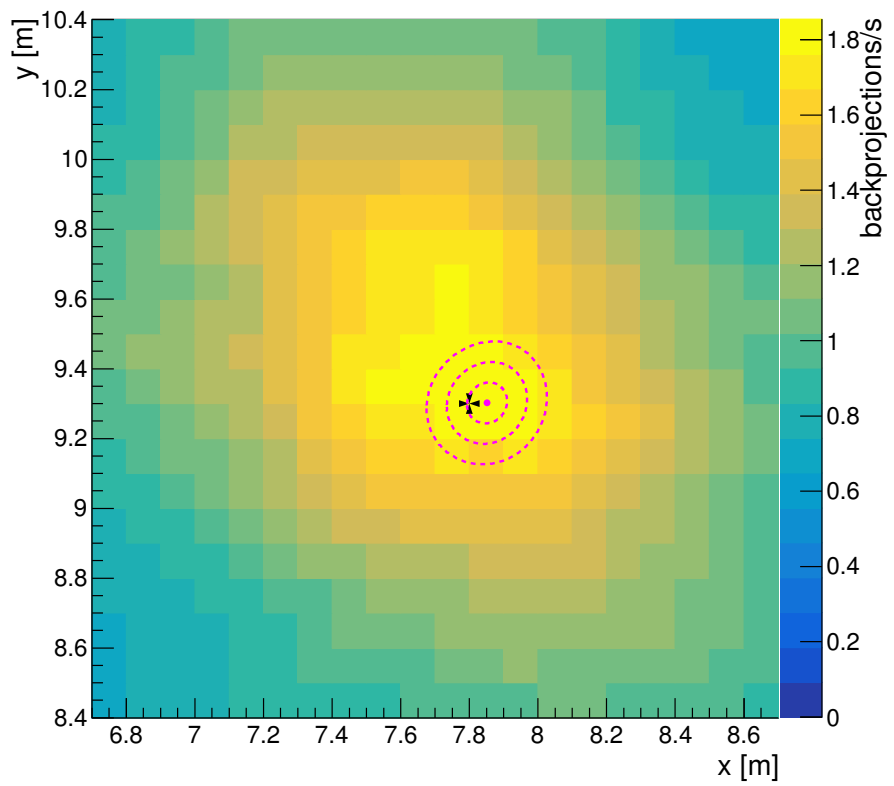

(b)

Figure 5.35: Tomographic reconstruction of experimental point source with pointresponse approximation overlaid. a) Full image space. b) Zoomed-in view at location of source. Red diamonds mark the dwell points, the point-model fit result has been overlaid with a magenta dot, and magenta dashed lines represent the $1 \sigma, 2 \sigma$, and $3 \sigma$ spatial contours. A black cross marks the true location of the point source. The temporal rate arises from normalization by measurement time at each dwell point. 


\subsubsection{Point-model estimate}

The point-model fit result has been overlaid on the tomographic results shown in figure 5.35 with a magenta dot and with $1 \sigma, 2 \sigma$, and $3 \sigma$ spatial contours shown by dashed magenta lines. The fit converged with a $\chi^{2}$ value of 6.5 for 6 degrees of freedom, placing a point source with a $662 \mathrm{keV}$ emission rate of $(15.2 \pm 0.2)$ million/s at coordinates $(7.9 \pm 0.1,9.3 \pm 0.1) \mathrm{m}$. The reported uncertainties only include statistical effects. The point-model fit took mere seconds to converge using a laptop computer with a $3.1 \mathrm{GHz}$ Intel Core i5 processor.

The location of the fitted point model agrees with the known location of the experimentally-measured point source. Comparison of the $662 \mathrm{keV}$ emission rate of the fit to the known $662 \mathrm{keV}$ emission rate of the source shows that the point-model fit underestimates the emission rate by $12.6 \%$ with a Z-score of -3.02 and a twosided P-value of 0.003 . The estimated $662 \mathrm{keV}$ emission rate of the point-model fit therefore does not agree with the known emission rate of the source at a significance level of 0.01. However, the estimated emission rate is consistent with the estimate produced by the spatial-unfolding method shown in section 5.2.3, and a discussion as to what may have caused the recovered activity to be underestimated is deferred to that section. The fact that the method works when applied to synthetic data generated using the same MC simulation model as the response simulations, as was shown in section 5.1.3, and not when applied to experimental data suggests that the simulated response has not included all the necessary facets of the real-world detector or experimental environment.

\subsubsection{Spatially-unfolded distribution}

The tomographic reconstruction methodology presented in section 4.5 and the spatialunfolding methodology described in section 4.8 were utilized for reconstruction of the experimentally-acquired point-source data. During the minimization process, it was assumed that there was no activity in the pixels outside of the combined field of view of all nine dwell points. The value of $w_{i}$ associated with these pixels was fixed to zero within the Minuit2 minimizer.

The tomographic reconstruction of the source of $662 \mathrm{keV}$ emissions in the $1 \mathrm{~m}$ 
by $1 \mathrm{~m}$ binning chosen for the unfolding is shown in figure 5.36a, with red diamonds denoting the positions of the dwell points and the true source location marked by a black cross. The tomographic reconstruction of the data indicates a region of maximal intensity at the true location of the point source, and demonstrates the spoking artefacts characteristic of tomographic back-projection.

Figure $5.36 \mathrm{~b}$ is the result of the fit of the simulated response templates to figure 5.36a. The fit took approximately 30 minutes to complete on a single $3.1 \mathrm{GHz}$ Intel Core i5 processor and reproduces much of the structure of the tomographic reconstruction; maximal intensity is placed at true location of the source, and the spoking artefacts are captured. This demonstrates the ability of the fit to capture the response of the system to the measured source. The agreement between the tomogram of the data and the tomographic fit can readily be observed by comparison of onedimensional profiles taken through the images. Figure 5.36c presents one-dimensional vertical profiles taken of the two-dimensional tomographic reconstruction of the data and the tomographic fit at $x=7.5 \mathrm{~m}$, while figure $5.36 \mathrm{~d}$ presents one-dimensional profiles along the horizontal defined by $y=9.5 \mathrm{~m}$. The profiles generally show good agreement within the estimated uncertainties, though the fit demonstrates a tendency to underestimate the intensity present at the location of the point source. The origin of this effect will be discussed later in this section.

The estimated distribution of $662 \mathrm{keV}$ gamma-ray emitters that results from unfolding the tomographic reconstruction of figure 5.36a is shown in figure 5.37a. The spatial-unfolding method has eliminated the spoking artefacts present in the tomographic reconstruction and has reduced the spatial extent of the reconstructed source to $2 \mathrm{~m}^{2}$, with a significant proportion of the reconstructed distribution accurately placed at the known location of the point source. The total emission rate of $662 \mathrm{keV}$ photons contained in the unfolded distribution is 14.4 million/s.

The statistical uncertainties on the result presented in figure 5.37a were estimated as described in section 4.8. For the repeated MC simulation method, 25 simulations of a point-source of monoenergetic $662 \mathrm{keV}$ photons with an emission rate of 14.4 million/s placed at the known location of the point source in the experimental geometry were run. This was done as opposed to taking the distribution shown in figure 5.37a because the source distribution was known in this experiment. For the bootstrapping 


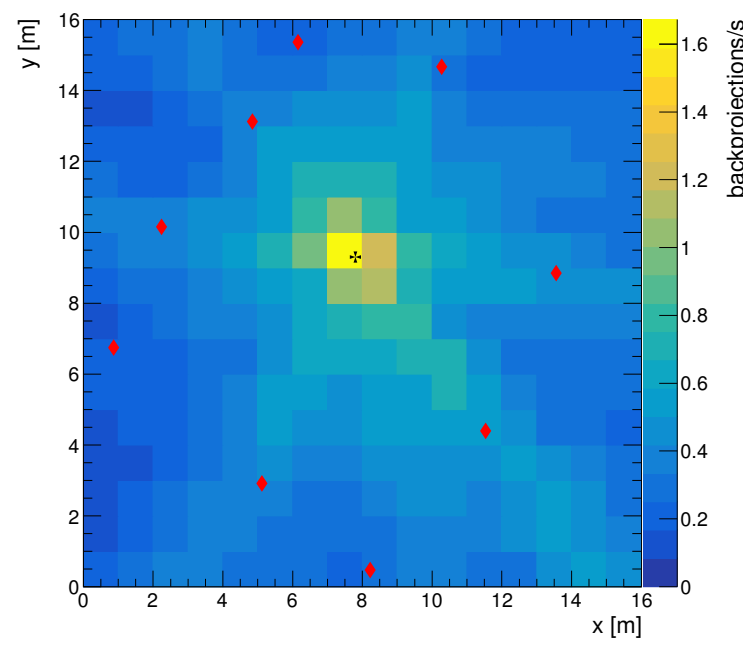

(a)

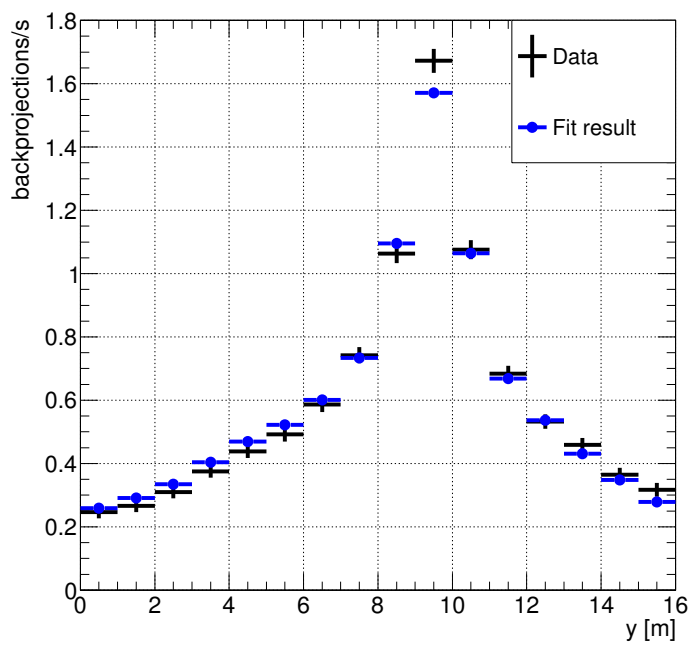

(c)

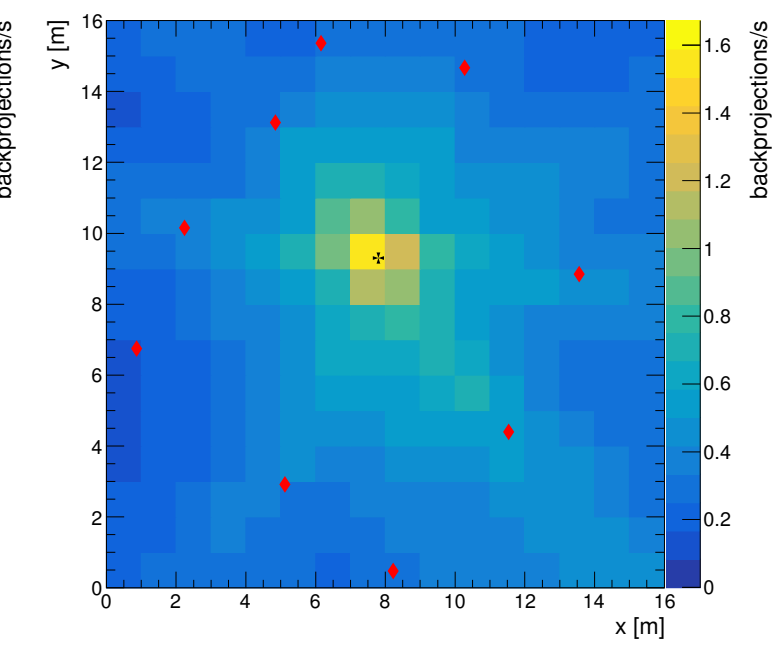

(b)

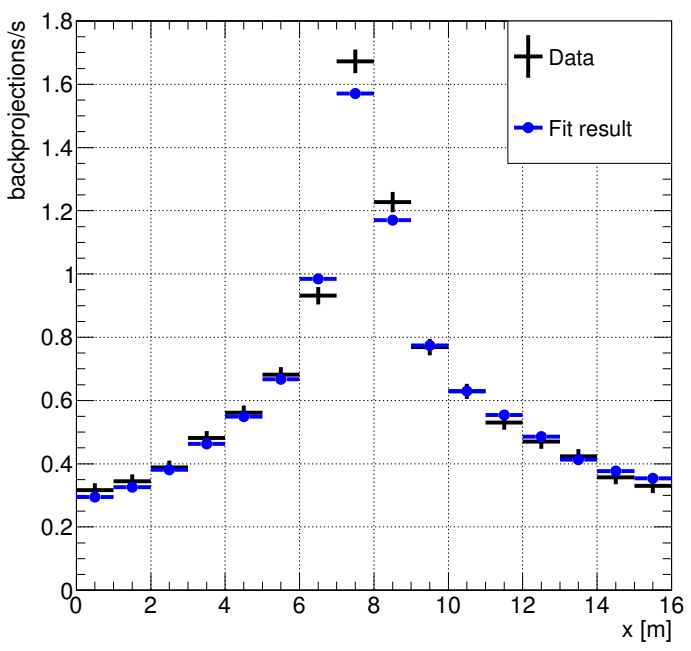

(d)

Figure 5.36: Tomographic reconstruction of experimentally-measured 662 ke V point source with fit to data tomogram produced by spatial unfolding. a) Tomographic reconstruction of point source. b) Fit to data tomogram produced by the minimization used in spatial unfolding. c) Vertical profiles of figures a and b taken at $x=7.5 \mathrm{~m}$. d) Horizontal profile of figures a and $b$ taken at $y=9.5 \mathrm{~m}$. The location of the source is marked by a black cross and red diamonds indicate the location of the dwell points in figures $a$ and $b$. Figures $a$ and $b$ are presented on a common colour scale.

method, 25 bootstrapped datasets were generated. Each of the datasets were then reconstructed, and the standard deviation of the images taken at each pixel as an estimate of the statistical uncertainty. Statistical uncertainty estimates generated 


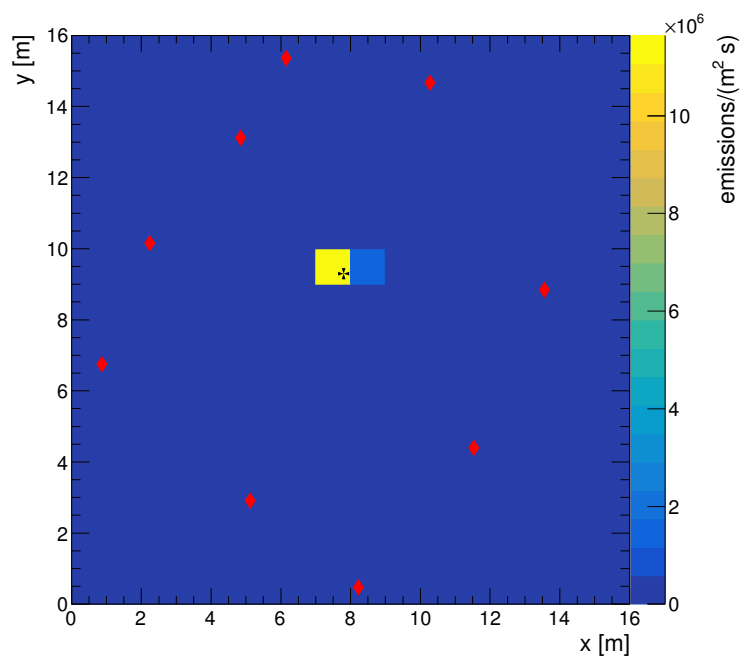

(a)

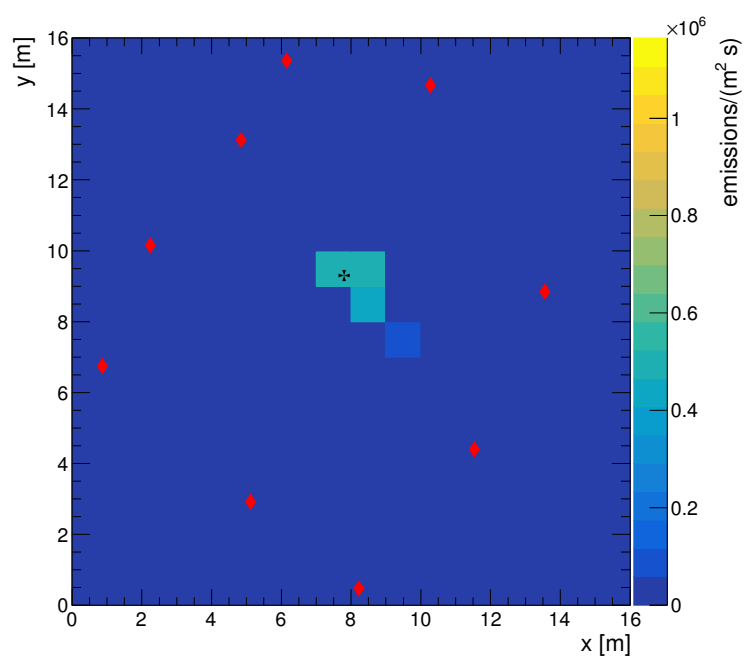

(b)

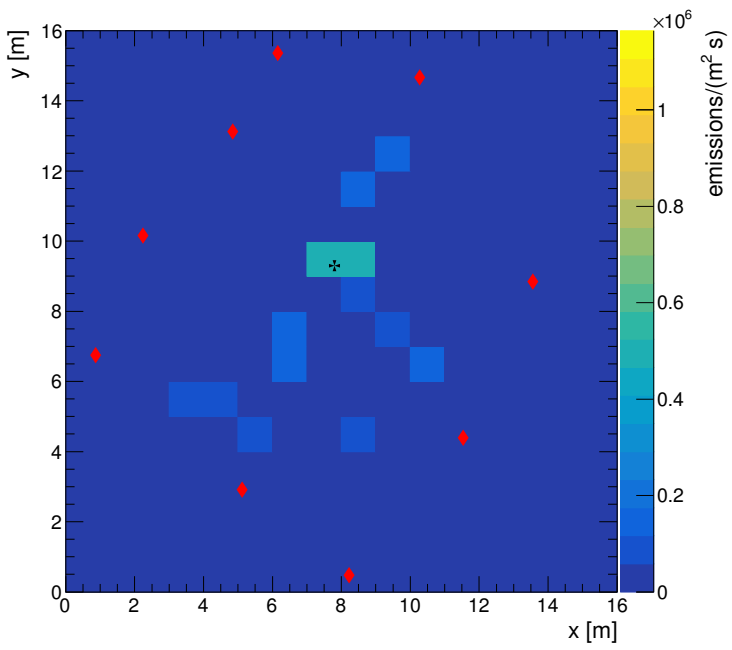

(c)

Figure 5.37: Unfolded distribution with statistical error estimates for experimentally-measured 662 keV point source. a) Estimated distribution of 662 keV photon emissions. b) Estimate of statistical uncertainty from the repeated MC simulation method. c) Estimate of statistical uncertainty from the bootstrapping method. The uncertainty maps are not visible at the scale of the unfolded image and are presented on a common lower scale. The location of the source is marked by a black cross and red diamonds indicate the location of the dwell points.

using the repeated MC simulation method are shown in figure 5.37b, whereas the statistical uncertainty estimates produced by the bootstrapping method are shown in figure 5.37c. The statistical uncertainty estimates are not visible on the same scale 
used to present the unfolded distribution and are therefore presented on a common lower scale. Both estimates of the statistical uncertainty show that the unfolded distribution of $662 \mathrm{keV}$ emissions is inconsistent with having no emissions at the true location of the source. Furthermore, the unfolded distribution taken together with the estimates of statistical uncertainty show that the placement of $662 \mathrm{keV}$ emissions away from the true location of the source is consistent with having no emissions at all. The spatial-unfolding method has therefore shown excellent ability to locate the source.

The total emission rate of $662 \mathrm{keV}$ photons predicted by the unfolding algorithm when applied to the experimentally-measured data was 14.4 million/s, with a statistical uncertainty of 0.8 million/s provided by both the repeated MC simulation method and the bootstrapping method. When compared to the expected emission rate of $(17.4 \pm 0.7)$ million/s this represents a $17.2 \%$ underestimation, with a Z-score of 2.82 and a two-sided P-value of 0.005 ; the values are inconsistent at a significance level of 0.01. However, the emission rate recovered by the point-model estimate given in section 5.2.2 is in agreement with the total emission rate in the spatially-unfolded distribution at a significance level of 0.01 as comparison of the two results yields a Z-score of 0.97 , corresponding to a two-sided P-value of 0.33 . The fact that both methods of source-strength estimation agree with one another suggests that there is an

effect missing from the response simulations that results in an underestimation of the emission rate of $662 \mathrm{keV}$ photons; this is further supported by the results presented in section 5.1.3, wherein the ability of the method to reproduce the proper emission rate was demonstrated on synthetic data. Effects not included in the simulated response of the detector are discussed later in this section.

\section{Investigation of the effect of using a "tile source" in reconstructing a point source}

The data are known to have originated from a point source, and yet the spatialunfolding method used $1 \mathrm{~m}$ by $1 \mathrm{~m}$ square response templates to represent the distribution of $662 \mathrm{keV}$ emissions inside the image space. The effect of this misrepresentation has been studied, and the results are presented in this section.

The fit shown in figure 5.36b to the tomographic reconstruction of the data pre- 
sented in figure 5.36a shows good overall agreement with the data, but demonstrates a tendency to underestimate the intensity present at the location of the point source. The representation of a point source by a $1 \mathrm{~m}$ by $1 \mathrm{~m}$ "tile source" is the cause of this phenomenon, and can be observed through investigation of synthetic data generated by $\mathrm{MC}$ simulation. A synthetic point source emitting $662 \mathrm{keV}$ photons at a rate of 17.4 million/s was simulated in the experimental geometry and was sampled with statistics similar to that in the experimental data. A tomographic reconstruction of this synthetic source is shown in figure 5.38a, and the fit achieved to the tomographic reconstruction is shown in figure $5.38 \mathrm{~b}$. The fit tomogram generally reproduces the patterns present in the data tomogram, but misrepresents the intensity of some of the pixels (e.g. the pixel at the true source location is underestimated in the fit). This is made clearer by the one-dimensional profiles shown in figures $5.38 \mathrm{c}$ and $5.38 \mathrm{~d}$, acquired vertically through $x=7.5 \mathrm{~m}$ and horizontally through $y=9.5 \mathrm{~m}$ respectively. The profiles show that the fit tomogram underestimates the maximum intensity of the data tomogram, corresponding to the location of the point source, and may under- or overestimate the data tomogram elsewhere. To demonstrate that this effect is not due to the number of statistics that were acquired, figure 5.39 demonstrates yet another set of tomographic results for a synthetic $662 \mathrm{keV}$ point source emitting at a rate of 17.4 million/s, but with 10 times the statistics contained in the results presented in figure 5.38. Even with higher statistics, the behaviour of the fit and its tendency to misrepresent the intensity of pixels within the data tomogram stays the same.

When the shape and size of the response templates are commensurate with that of the underlying source distribution, the tomographic fit quality improves. A $1 \mathrm{~m}$ by $1 \mathrm{~m}$ uniformly-emitting "tile source" of $662 \mathrm{keV}$ photons with an emission rate of 17.4 million/s was simulated in MC, acquiring statistics similar to those of the experimental data and of the synthetic results presented in figure 5.38. Figure 5.40a shows the tomographic reconstruction of this synthetic tile source, and figure $5.40 \mathrm{~b}$ is the result of the fit of the simulated response templates to figure 5.40a; in each figure, dashed black lines mark the true location of the source. The data tomogram and the fit tomogram now agree within the estimated uncertainties, a fact which is once again made clear by inspection of one-dimensional profiles of each image. Figure 5.40c shows vertical profiles taken at $x=7.5 \mathrm{~m}$ whereas figure $5.40 \mathrm{~d}$ shows horizontal profiles taken at $y=9.5 \mathrm{~m}$. Both sets of profiles demonstrate the agreement between the 


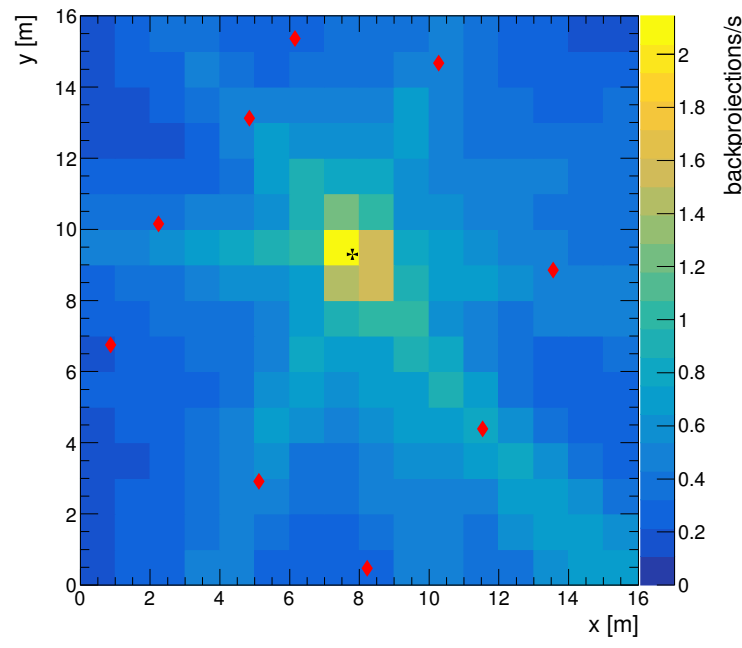

(a)

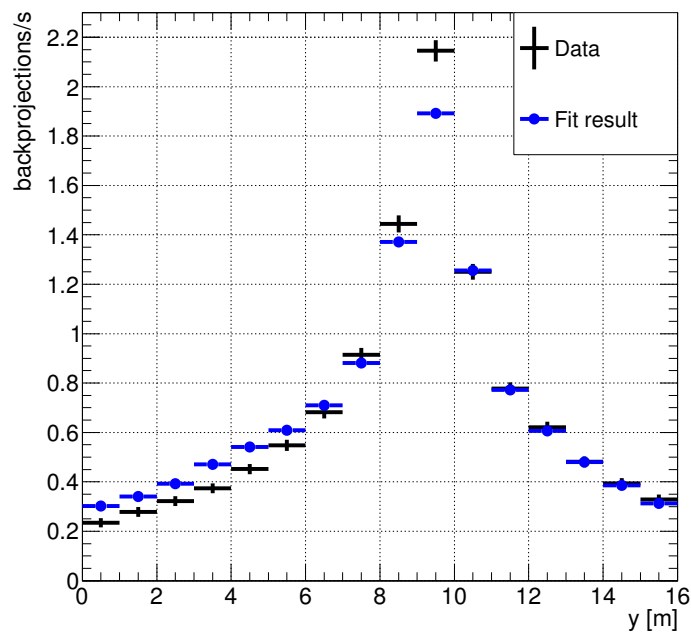

(c)

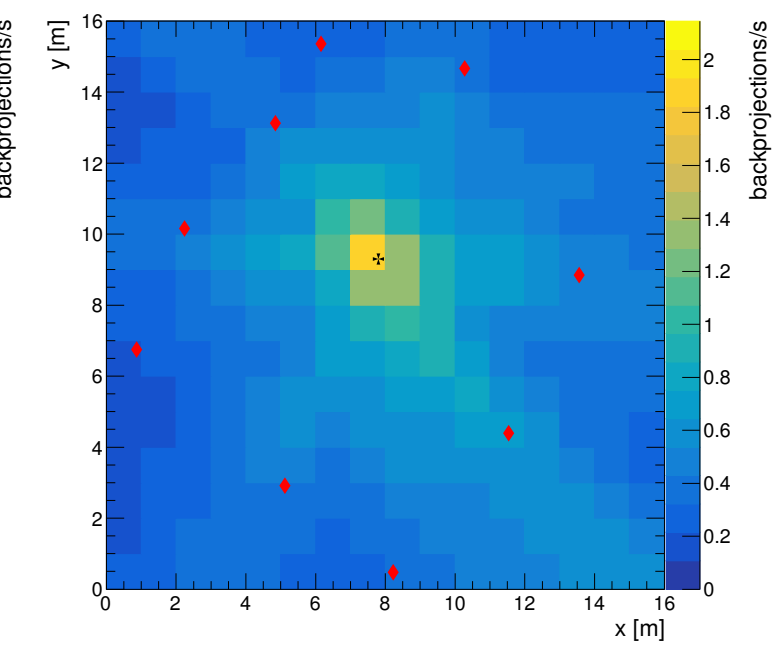

(b)

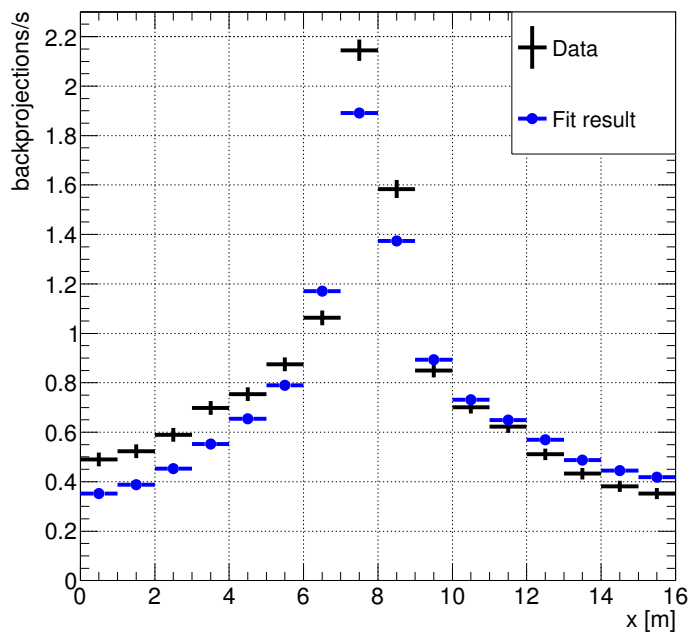

(d)

Figure 5.38: Tomographic reconstruction of 17.4 million emission/s synthetic 662 keV point source with fit to data tomogram produced by spatial unfolding, with similar statistics to that of the experimental data. a) Tomographic reconstruction of point source. b) Fit to data tomogram produced by the minimization used in spatial unfolding. c) Vertical profiles of figures $a$ and $b$ taken at $x=7.5 \mathrm{~m}$. d) Horizontal profile of figures $a$ and $b$ taken at $y=9.5 \mathrm{~m}$. The location of the source is marked by a black cross and red diamonds indicate the location of the simulated dwell points in figures $a$ and $b$. Figures $a$ and $b$ are presented on a common colour scale.

fit tomogram and the data tomogram. The fit quality is improved as the statistical power of the measurements increase. Figure 5.41 shows tomographic reconstruction and fit results for the synthetic tile source with 10 times the statistics of the results 


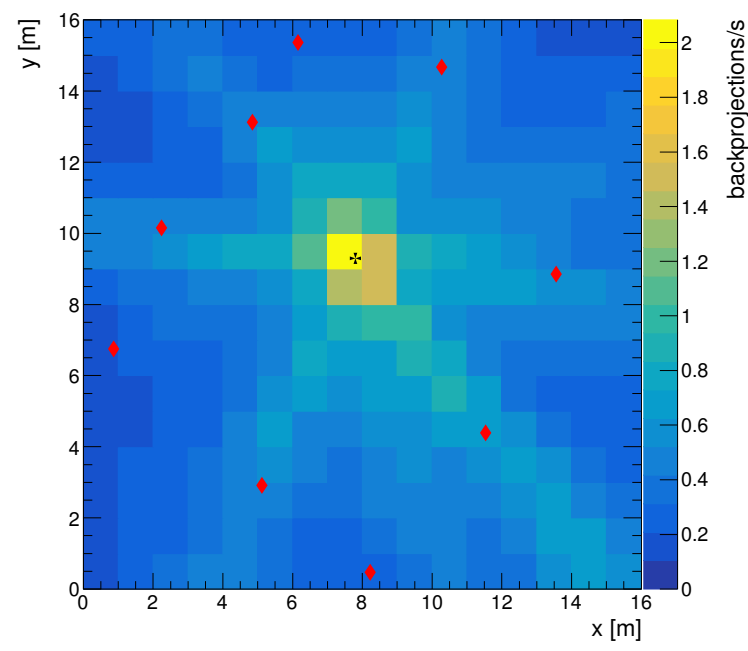

(a)

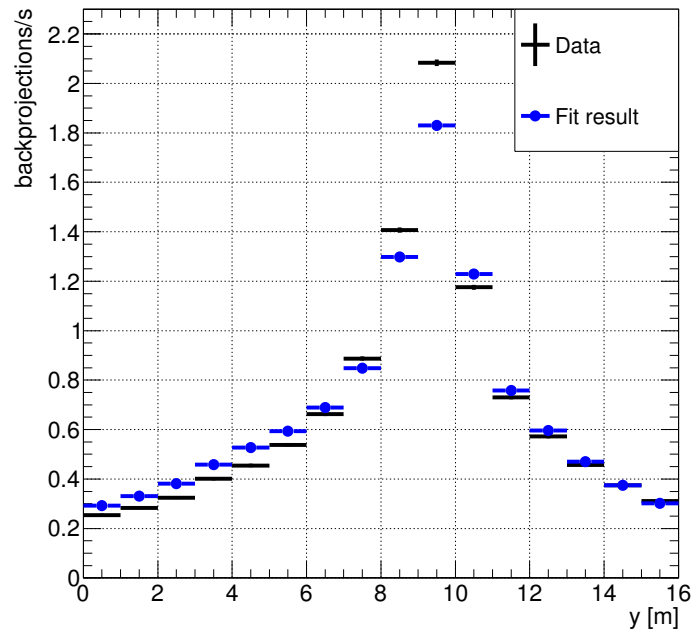

(c)

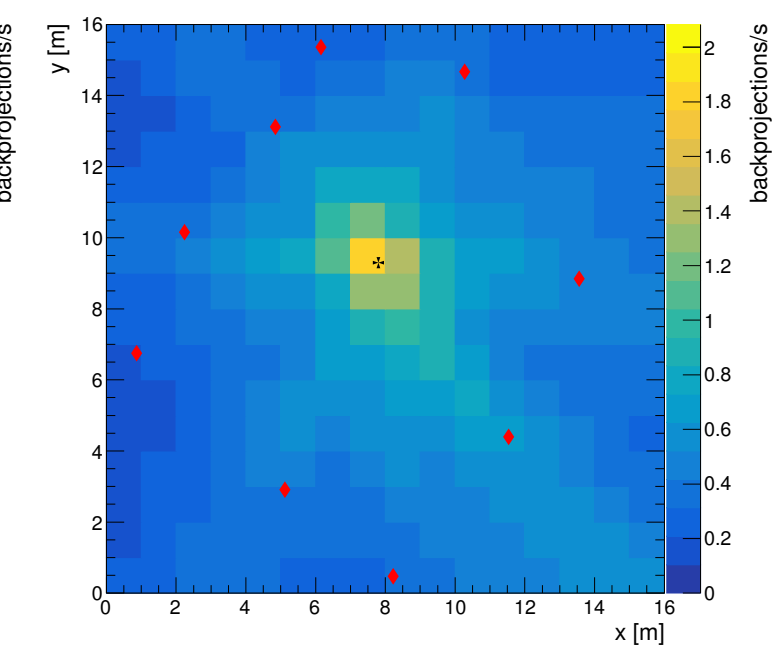

(b)

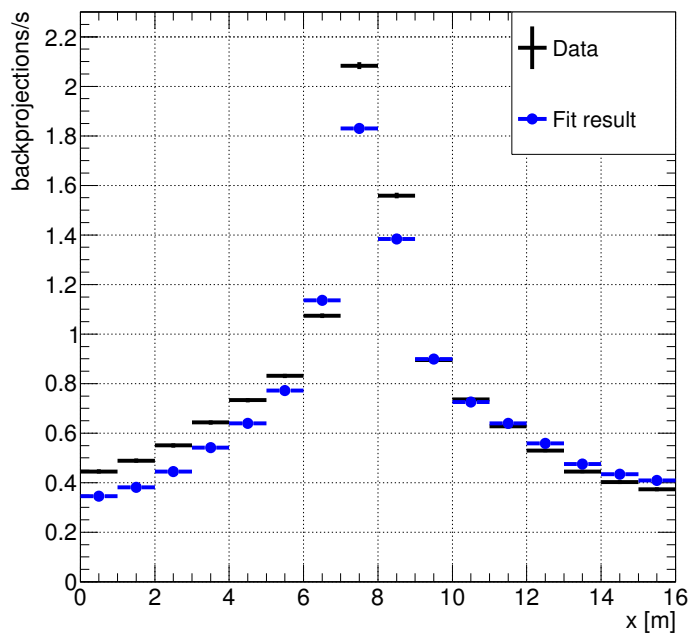

(d)

Figure 5.39: Tomographic reconstruction of 17.4 million emission/s synthetic $662 \mathrm{keV}$ point source with fit to data tomogram produced by spatial unfolding, with 10 times the statistics of the experimental data. a) Tomographic reconstruction of point source. b) Fit to data tomogram produced by the minimization used in spatial unfolding. c) Vertical profiles of figures $a$ and $b$ taken at $x=7.5 \mathrm{~m}$. d) Horizontal profile of figures $a$ and $b$ taken at $y=9.5 \mathrm{~m}$. The location of the source is marked by a black cross and red diamonds indicate the location of the simulated dwell points in figures $a$ and $b$. Figures $a$ and $b$ are presented on a common colour scale.

shown in figure 5.40, along with one-dimensional profiles extracted along the same coordinates as were shown in figure 5.40. The fit has nearly perfectly reproduced the tomographic reconstruction of the data. 


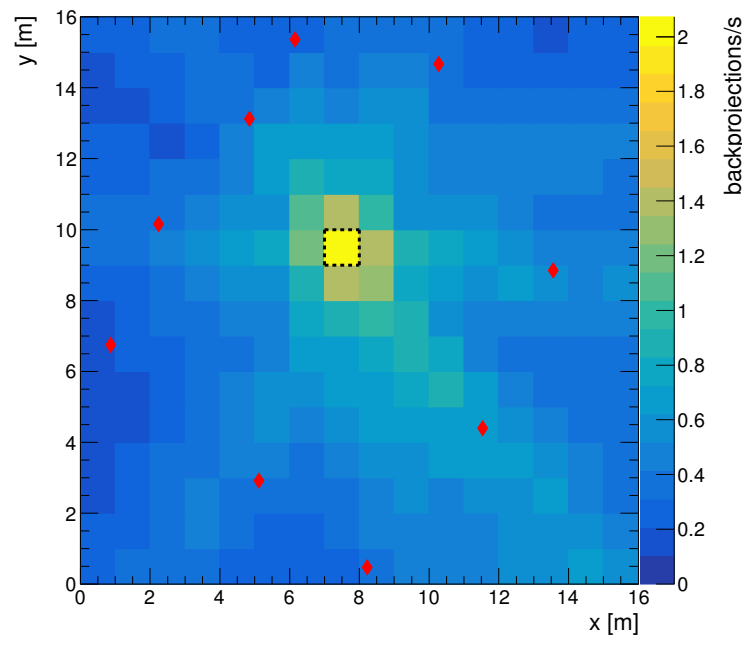

(a)

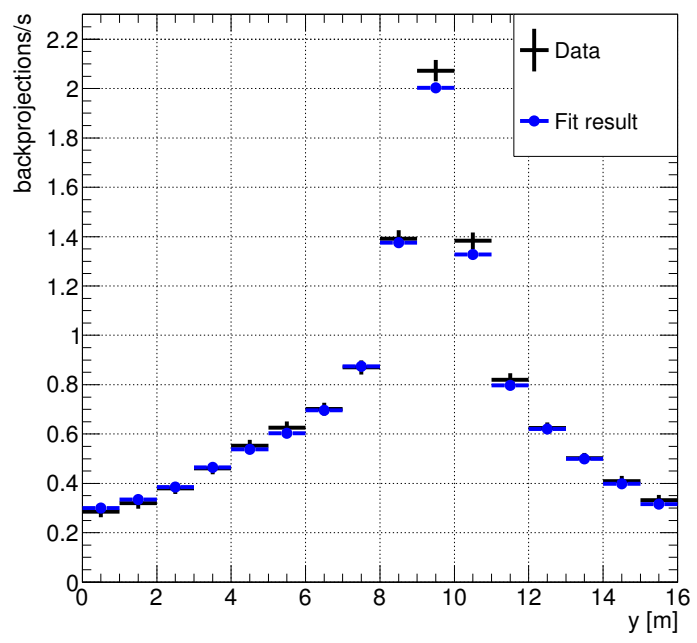

(c)

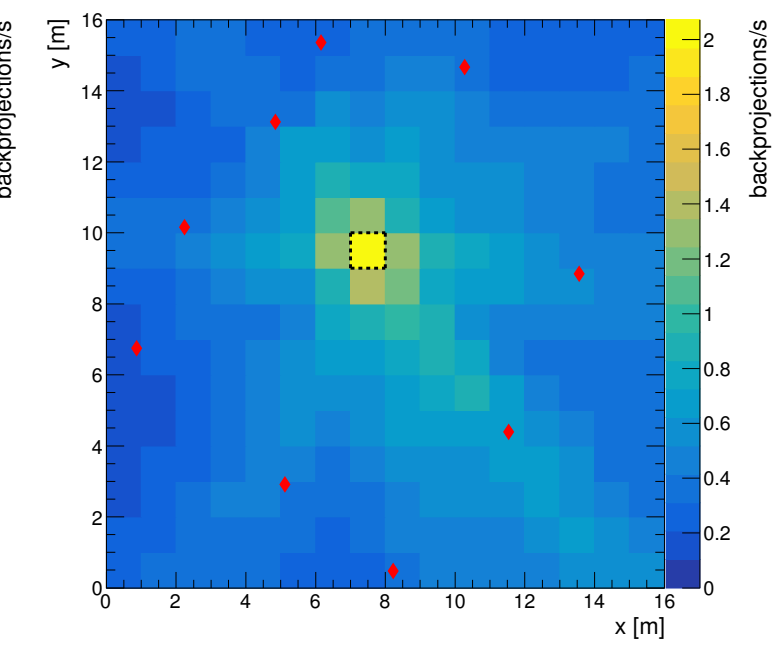

(b)

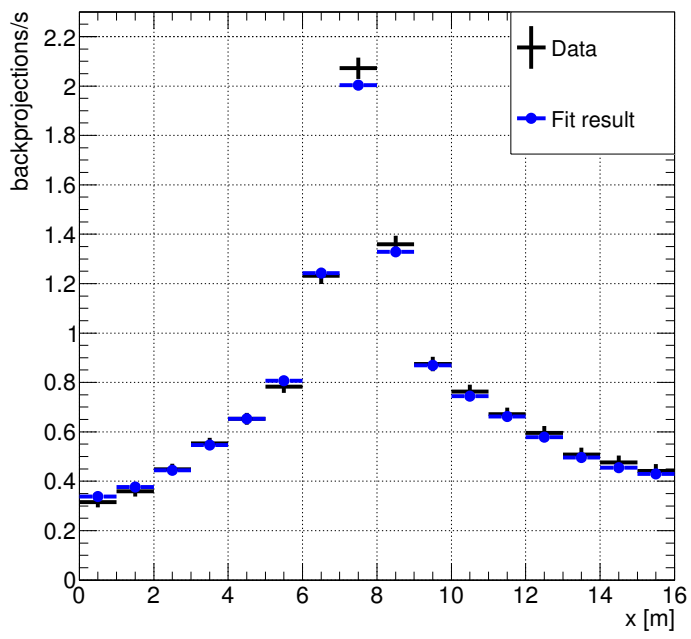

(d)

Figure 5.40: Tomographic reconstruction of 17.4 million emission/s synthetic $1 \mathrm{~m}$ by $1 \mathrm{~m}$ 662 keV "tile source" with fit to data tomogram produced by spatial unfolding, with similar statistics to that of the experimental data. a) Tomographic reconstruction of tile source source. b) Fit to data tomogram produced by the minimization used in spatial unfolding. c) Vertical profiles of figures $a$ and $b$ taken at $x=7.5 \mathrm{~m}$. d) Horizontal profile of figures $a$ and $b$ taken at $y=9.5 \mathrm{~m}$. The location of the source is marked by black dashed lines and red diamonds indicate the location of the simulated dwell points in figures $a$ and $b$.

Figures $5.42 \mathrm{a}$ and $5.42 \mathrm{c}$ show the unfolded distributions corresponding to the "tile-source" fits presented in figures 5.40b and 5.41b, respectively. Both reconstructions place the activity in the response template containing the "tile source" and 


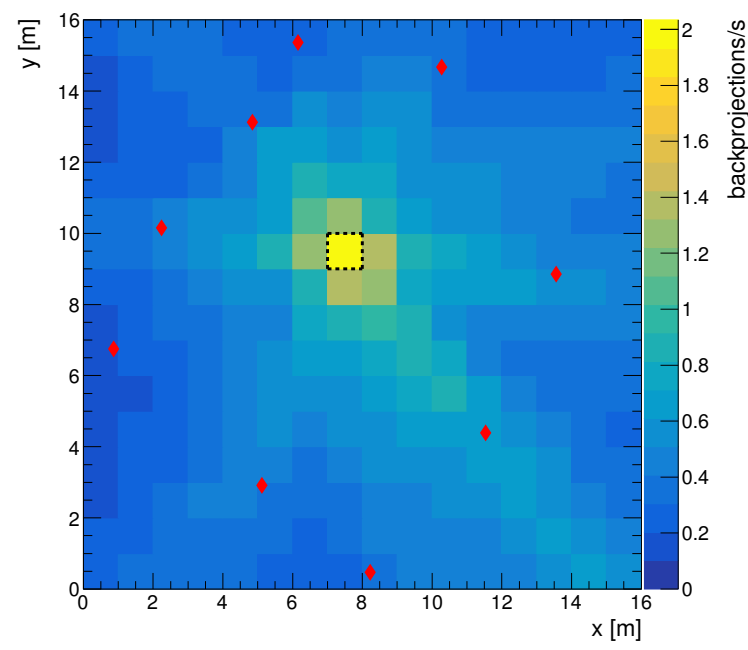

(a)

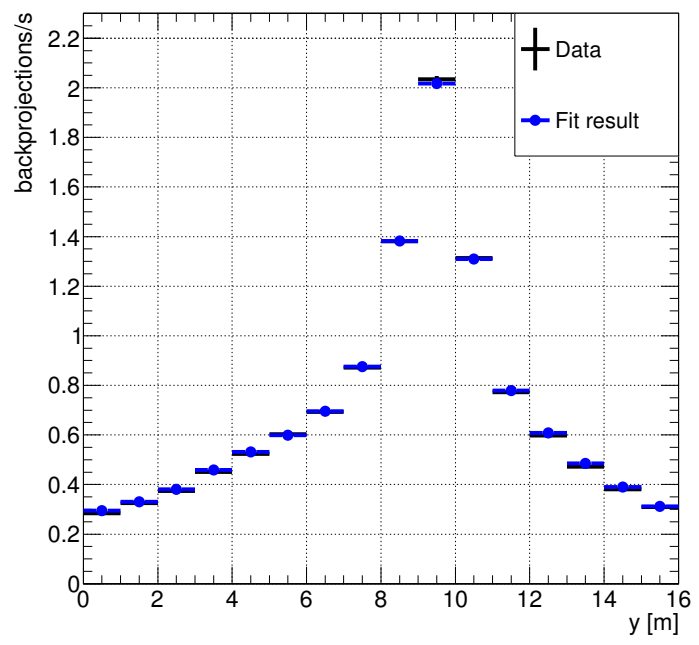

(c)

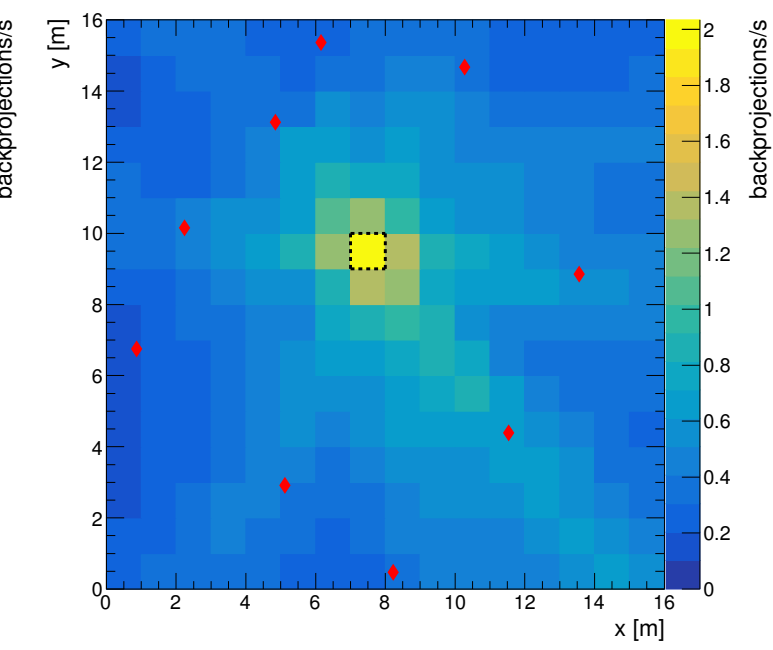

(b)

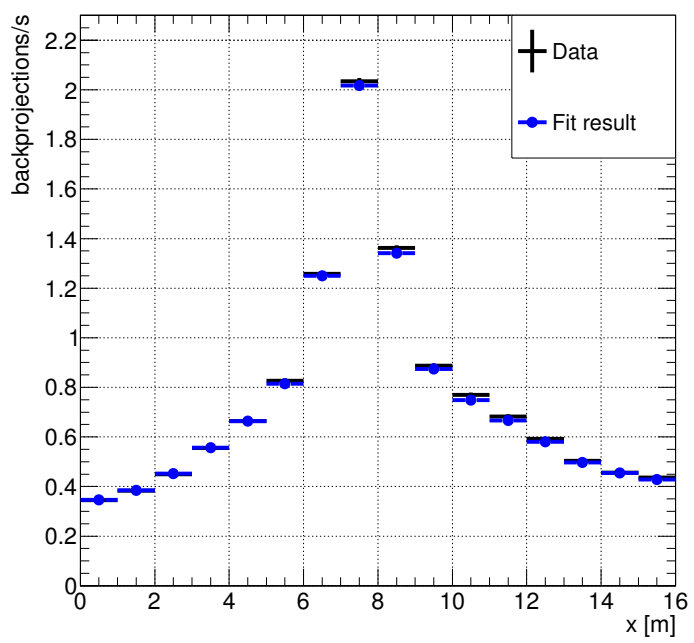

(d)

Figure 5.41: Tomographic reconstruction of 17.4 million emission/s synthetic $1 \mathrm{~m}$ by $1 \mathrm{~m} 662 \mathrm{keV}$ "tile source" with fit to data tomogram produced by spatial unfolding, with 10 times the statistics of the experimental data. a) Tomographic reconstruction of tile source source. b) Fit to data tomogram produced by the minimization used in spatial unfolding. c) Vertical profiles of figures a and b taken at $x=7.5 \mathrm{~m}$. d) Horizontal profile of figures $a$ and $b$ taken at $y=9.5 \mathrm{~m}$. The location of the source is marked by black dashed lines and red diamonds indicate the location of the simulated dwell points in figures $a$ and $b$.

reconstruct total emission rates of 17.2 million/s and 17.3 million/s, respectively, representing $-1.1 \%$ and $-0.6 \%$ deviations from the expected value. These results demonstrate that the spatial-unfolding method can properly recover a source whose shape 
and size is commensurate with the response templates used in the fitting procedure.

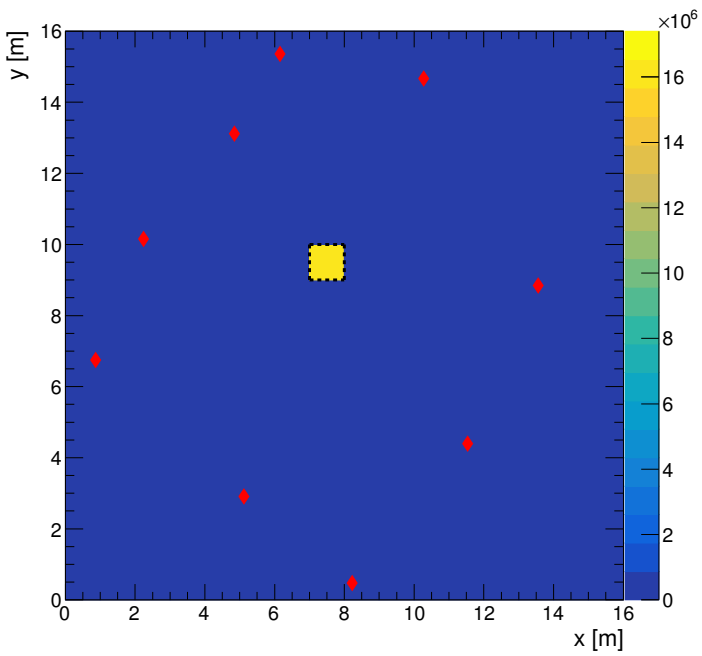

(a)

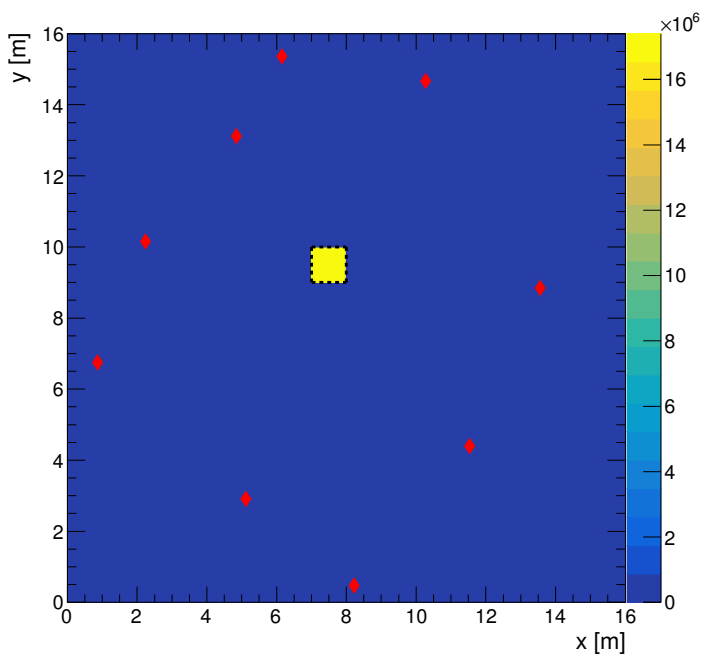

(c)

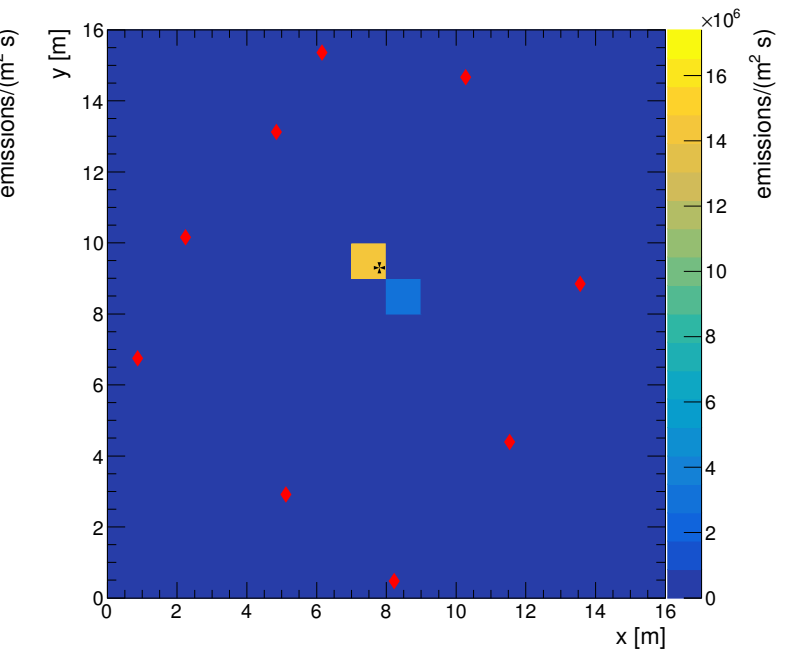

(b)

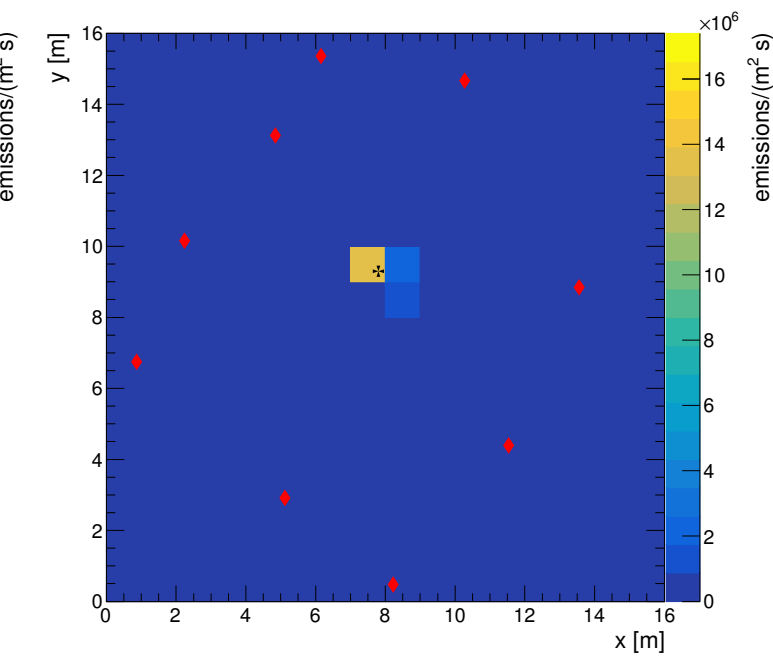

(d)

Figure 5.42: Comparison of spatial-unfolding results for synthetic data for sources with 662 keV emission rates of 17.4 million/s. a) $1 \mathrm{~m}$ by $1 \mathrm{~m}$ tile source with statistics equal to those of the experimental data. b) Point source with statistics equal to those of the experimental data. c) $1 \mathrm{~m}$ by $1 \mathrm{~m}$ tile source with 10 times the statistics of the experimental data. d) Point source with 10 times the statistics of the experimental data. Subfigures have been presented on a common colour scale, and black cross/dashed lines mark placement of synthetic source.

Misrepresentation of the point source by a set of $1 \mathrm{~m}$ by $1 \mathrm{~m}$ "tile-source" response 
templates does not, however, destroy the ability of the spatial-unfolding method to both localize and quantify the source. Figures 5.42b and 5.42d show the unfolded distributions corresponding to the point-source fits presented in figures 5.38b and $5.39 \mathrm{~b}$, respectively. The unfolded distributions corresponding to the synthetic point

sources demonstrate "bleeding" of emissions into pixels adjacent to that containing the point source, but have still removed the spoking artefacts present in the original data tomogram. In particular, the point source sits towards the lower right of the pixel that contains it, and it is the pixel to the immediate right and below and to the right which contain the majority of the dispersed emissions. The "bleeding" which results from approximating a point source by a basis of $1 \mathrm{~m}$ by $1 \mathrm{~m}$ tile sources extends only into the pixels adjacent to the the pixel which truly contains the source and in a predictable manner, thereby reducing the space in which a search would need to be made to find the source of the gamma rays. Additionally, the total emission rate reconstructed in the image space closely approximates the expected value of 17.4 million/s. Total emission rates of 17.5 million/s and 17.0 million/s were reconstructed in figures $5.42 \mathrm{~b}$ and $5.42 \mathrm{~d}$, respectively, representing deviations of $0.6 \%$ and $-2.3 \%$ from the expected value. Thus, the spatial-unfolding method reasonably captures the underlying rate of gamma-ray emissions, even when the shape of the response templates cannot perfectly reproduce the underlying distribution of radioactive material.

\section{Effects not included in simulated detector response}

Both the point-response method and the spatial-unfolding method demonstrate excellent source-localization abilities, and produce estimates of the source emission rate that are in agreement with each other but that are not in agreement with the expected emission rate. This suggests that the simulated response of the detector does not include all the effects of the real-world detector. Efforts to improve agreement are underway. Possible explanations being investigated for the discrepancy include missing or poorly-modelled dead materials, trigger threshold effects, electronic noise, and overlapping events. 


\subsection{Summary}

The tomographic reconstruction of experimental data presented in section 5.2.1 takes only minutes to complete, and demonstrates good localization of the $662 \mathrm{keV}$ point source with known tomographic spoking artefacts. Operators who are familiar with the properties of tomographic images would readily be able to locate the source in near real time with the tomographic reconstruction of the data presented in figure 5.35. This supports hypothesis number 2 listed in section 1.2.

The point-model parametrization demonstrates good localization of the experimental point source, but underestimates the emission rate of $662 \mathrm{keV}$ photons. However, the method was shown to predict the emission rate of $662 \mathrm{keV}$ photons accurately when applied to synthetic data generated by MC simulations, and it is presumed that the simulated detector response does not capture all effects that may lead to a loss of signal in the real world. The ability of the method to locate an experimental point source accurately and to recover the emission rate of a synthetic point source supports hypothesis number 3 listed in section 1.2.

The spatial-unfolding methodology successfully locates the experimentallymeasured point source, and its estimated $662 \mathrm{keV}$ emission rate agrees with the emission rate predicted by the point-model method. Application of the spatial-unfolding method to synthetic "tile-source" and point-source data demonstrates that the tomographic fit is most accurate when the response templates are commensurate in size and shape to the underlying source, but that the method still reasonably reproduces the emission rate of a source whose distribution is not commensurate with the size and shape of the response templates. The synthetic results, along with the agreement of the spatially-unfolded result and the point-model result, suggests that the disparity between the predicted $662 \mathrm{keV}$ photon emission rate and the expected value results from an effect not captured by the response template simulations. Taken together, the experimental and synthetic results presented in this chapter for the spatial-unfolding method lend support to hypothesis number 4 of section 1.2. 


\section{Chapter 6}

\section{Spatially-extended source trial}

This chapter presents an experiment wherein a spatially-extended source of La-140 was measured in outdoor operational conditions. The trial took place at Canadian Forces Base Suffield in Alberta, in partnership with Defence Research and Development Canada (DRDC). The methodology specific to this trial is outlined, and results from the trial are presented and discussed.

\subsection{Methodology}

\subsubsection{Experimental setup}

The experiment took place outdoors in March of 2018 at Canadian Forces Base Suffield, Alberta, at the DRDC Suffield Research Centre. Figure 6.1 provides a schematic representation of the experimental scenario, presented in Universal Transverse Mercator (UTM) easting and northing with an arbitrary offset applied to make the coordinates more convenient. A $500 \mathrm{~m}$ by $500 \mathrm{~m}$ restricted-access zone, denoted by solid black lines in figure 6.1, was defined within which the 3x3-module SCoTSS detector could not enter. An area measuring approximately $350 \mathrm{~m}$ by $170 \mathrm{~m}$ inside of the restricted-access zone was cleared of snow, within which a distributed source of La-140 in the shape of an "L" polygon was deposited. The L-polygon measured $120 \mathrm{~m}$ by $20 \mathrm{~m}$ on the large arm and $10 \mathrm{~m}$ by $80 \mathrm{~m}$ on the small arm, where the $80 \mathrm{~m}$ length includes a $20 \mathrm{~m}$ overlap with the large arm. The deposition of the La-140 source is discussed in detail further below. 


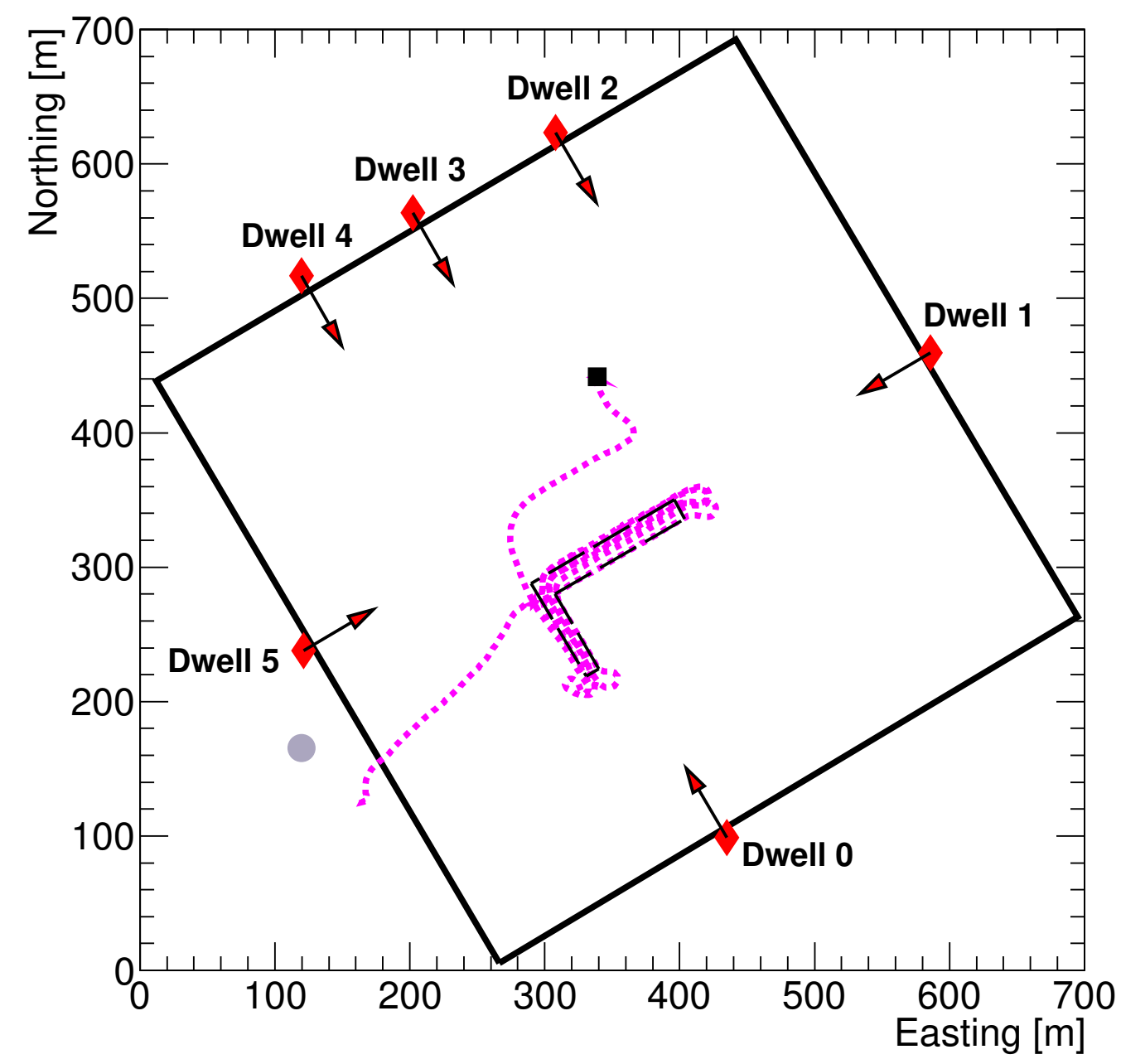

Figure 6.1: Schematic representation of the Suffield experimental site, shown in UTM easting and northing with arbitrary offset applied. The restricted-access zone has been marked by solid black lines, and dashed black lines outline the intended placement of the La-140. The path taken by the sprayer UGV has been marked by the dashed magenta line, while the solid black square indicates the final resting position of the vehicle. Red diamonds mark the locations of the dwell points used to image the La-140 on March 22, 2018, while the accompanying red arrows indicate the estimated yaw angle of the detector. The grey circle marks the location at which a measurement of the NORM background was taken on March 19th, 2018. 
A day prior to the deposition of the La-140, a measurement of the NORM background was acquired from a single location at the south-west side of the restrictedaccess zone over a period of 2820.78 s. The grey circle within figure 6.1 indicates the position of the NORM background measurement. Independent measurements of the distribution of NORM background within the restricted-access zone acquired by an unmanned aerial vehicle (UAV) equipped with a gamma spectrometer indicate that the background was reasonably uniform at the energy of the $1.596 \mathrm{MeV}$ La-140 photopeak. ${ }^{76}$ The single NORM background measurement acquired at the south-west side of the zone was therefore assumed to be representative of the NORM background at all locations around the perimeter of the restricted-access zone.

The deposition of the La-140 took place on March 20th, 2018. For full details on the deposition process, see reference 77. The salient points will be repeated here. An aqueous solution containing La-140 and a blue dye to assist in visualization of the source was prepared, and at 15:07 MDT on March 20th, 2018, there was 35 GBq of La-140 present. An unmanned ground vehicle (UGV) equipped with standard agricultural spraying equipment was used to deposit the La-140 solution, guided by an on-board autopilot unit that drove the UGV through a pre-programmed GNSS path for the planned placement of the L-polygon. Figure 6.2 shows the sprayer UGV as it deposited La-140, where the La-140 is visible as a blue shade beneath the spray arm at the rear of the vehicle. Dashed black lines in figure 6.1 indicate the intended placement of the L-polygon. The original intent was to deposit a surface activity concentration of $10 \mathrm{MBq} / \mathrm{m}^{2}$ of La-140, however, mechanical problems with the spraying equipment forced an impromptu change to the planned La-140 deposition which resulted in extra activity being placed in the large arm of the L-polygon. The UGV autonomously drove through its pre-determined course, which has been traced in figure 6.1 using a dashed magenta line, and the on-board sprayer was manually turned off by DRDC scientists when the vehicle was turning around outside of the borders of the planned L-polygon pattern. Once its tank had been emptied, the UGV was driven approximately $120 \mathrm{~m}$ away from the distributed La-140 towards the northern end of the restricted-access zone. The final position of the sprayer UGV is indicated by a black square in figure 6.1 . 


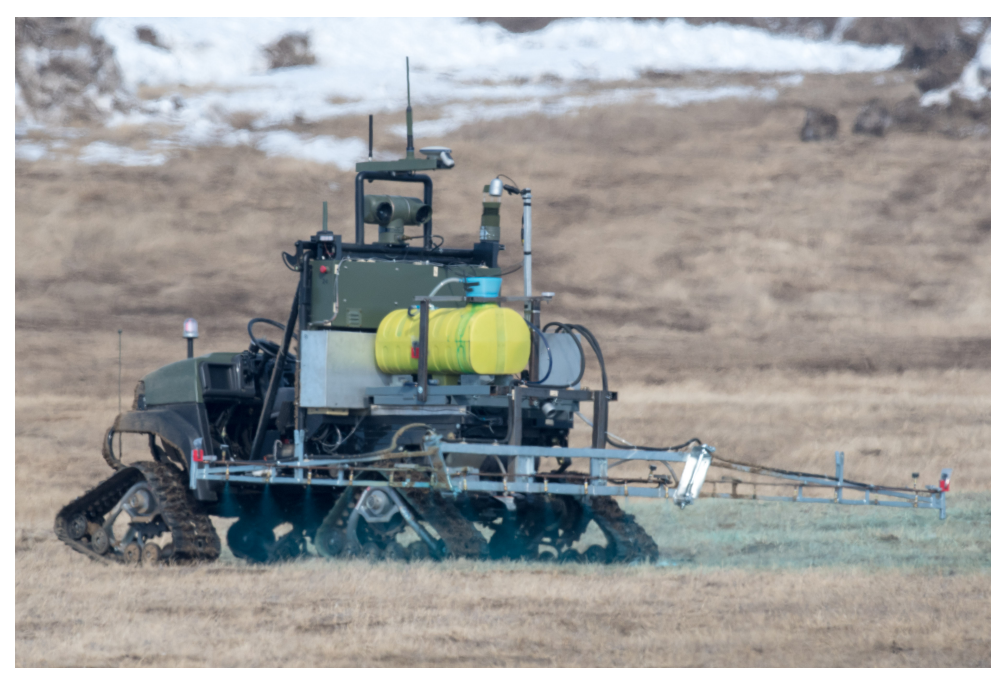

Figure 6.2: Sprayer UGV used to deposit the source during the Suffield experiment, pictured during deposition of La-140. Photo taken by Mike Franz, DRDC Suffield Research Centre.

\subsubsection{Experimental data collection}

Measurements of the distributed La-140 source using the 3x3-module SCoTSS detector were made two days after the initial deposition. Due to the relatively short half-life of La-140 of 40.29 hours, approximately 15 GBq of La-140 remained at the time of measurement. The 3x3-module SCoTSS detector was mounted on top of a Hägglunds Bandvagn 206 (BV206) for transportation around the perimeter of the restricted-access zone. Figure 6.3 shows the 3x3-module SCoTSS detector in position atop the BV206 vehicle, where a red ellipse indicates the detector for clarity. The central plane of the detector sat approximately $2.5 \mathrm{~m}$ above ground level in this configuration. The detector was secured in place on the rear carriage of the vehicle facing perpendicularly to the carriage and towards the left side of the vehicle when viewed from the rear.

The BV206 was driven in a counter-clockwise path around the perimeter of the restricted-access zone, beginning at the first dwell point at the south-east of the zone and finishing at the last dwell point at the south-west of the zone. An effort was made to drive the BV206 parallel to the borders of the restricted-access zone at all times. Data were acquired at a total of six dwell points, indicated by red diamonds within figure 6.1 and labelled from zero to five. Table 6.1 lists the start time and measurement duration for each dwell point; this information was used to apply a 


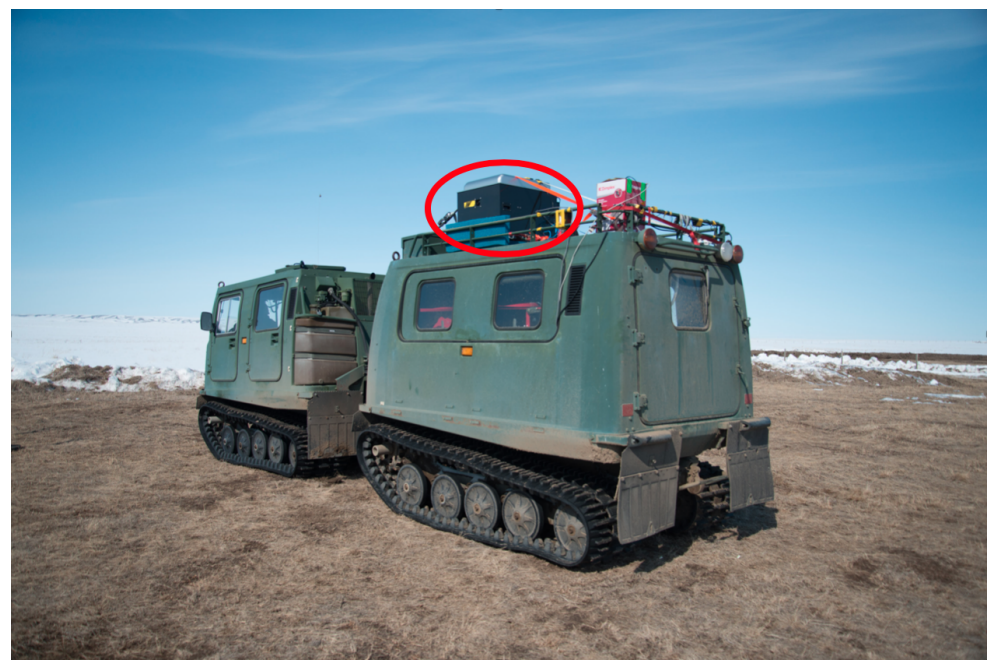

Figure 6.3: Transportation of the 3x3-module SCoTSS detector using a BV206 tracked vehicle. The detector has been indicated by a red ellipse. Photo taken by Mike Franz, DRDC Suffield Research Centre.

decay correction via equation 4.7 to the measurements acquired at each dwell point when reconstructing tomographic images in order to bring the equivalent activity back to what it would have been at 15:07 MDT on March 20th, 2018, when 35 GBq of La140 was present. The location and yaw angle of the 3x3-module SCoTSS detector at

Table 6.1: Start times and measurement durations for experimental dwell-point measurements acquired on March 22nd, 2018.

\begin{tabular}{c|c|c} 
Dwell point & Measurement start time (MDT) & Measurement duration (s) \\
\hline 0 & $13: 02: 29$ & 764.07 \\
1 & $13: 20: 37$ & 868.25 \\
2 & $13: 40: 40$ & 629.39 \\
3 & $13: 54: 25$ & 730.59 \\
4 & $14: 10: 12$ & 794.79 \\
5 & $14: 28: 37$ & 981.96
\end{tabular}

each dwell point were estimated using information provided by the on-board GNSS; the yaw angles have been indicated by red arrows in figure 6.1. The procedure for estimating the location and yaw angle of the detector at each dwell point is described in section 6.1.3.

Environmental conditions during the measurements were not recorded, and were estimated from archived records maintained by Environment Canada for Ralston, 
Alberta (approximately $23 \mathrm{~km}$ from the experimental site) and in Medicine Hat, Alberta (approximately $35 \mathrm{~km}$ from the experimental site), which provided measurements of the air temperature and air pressure, respectively*. The air temperature in Ralston varied between $0{ }^{\circ} \mathrm{C}$ and $2{ }^{\circ} \mathrm{C}$ over the time period of the La-140 measurements, whereas the atmospheric pressure fluctuated between $92.4 \mathrm{kPa}$ and $92.7 \mathrm{kPa}$ in Medicine Hat. Air humidity was not considered.

Of note is that one day prior to the experimental measurements analysed and presented in this thesis, preparatory measurements were attempted with the $3 \times 3$-module SCoTSS detector mounted atop the BV206 and transported around the restrictedaccess zone. However, vibrations from the vehicle caused the bolts holding the absorber plane in place to shake loose and the absorber plane to fall out of its supports. The absorber plane was remounted and all of the bolts supporting each detector plane were secured in place with an adhesive. The original $20 \mathrm{~cm}$ interplane gap was reproduced in this process to within $0.5 \mathrm{~cm}$ following the repair.

\section{Data quality}

Figure 6.4 shows total-energy spectra for two-hit events at dwell point 0. Figure 6.4a shows the La-140 + NORM background spectrum measured at dwell point 0 in black and the NORM background spectrum in blue, where the background spectrum has been scaled as described in section 4.3 using a lower energy limit of $1.75 \mathrm{MeV}$. Figure 6.4b shows the background-subtracted La-140 spectrum measured at dwell point 0, where the inset shows a zoomed-in view past the lower energy limit used in the scaling process. Figures 6.5 to 6.9 show similar spectra for dwell points 1 to 5 . The background subtraction was generally good, evidenced by the agreement of the shape of the NORM background spectrum with the shape of the La-140 + NORM background past the normalization threshold of $1.75 \mathrm{MeV}$ and additionally by the background-subtracted La-140 spectra shown for each dwell point. There are potential signs of over-subtraction of the $1.46 \mathrm{MeV}$ K-40 peak at dwell points 0,1 , and 3 , and of over-subtraction of the $0.609 \mathrm{MeV}$ U-238 peak at dwell point 4 . The use of a single background measurement to represent the background at all dwell points is nevertheless similar to what may have to be done in a real application, wherein a

*Unfortunately, Ralston did not provide a complete set of data, necessitating the additional use of the air pressure data from Medicine Hat. 
nearby non-contaminated area may have to serve as a proxy. 


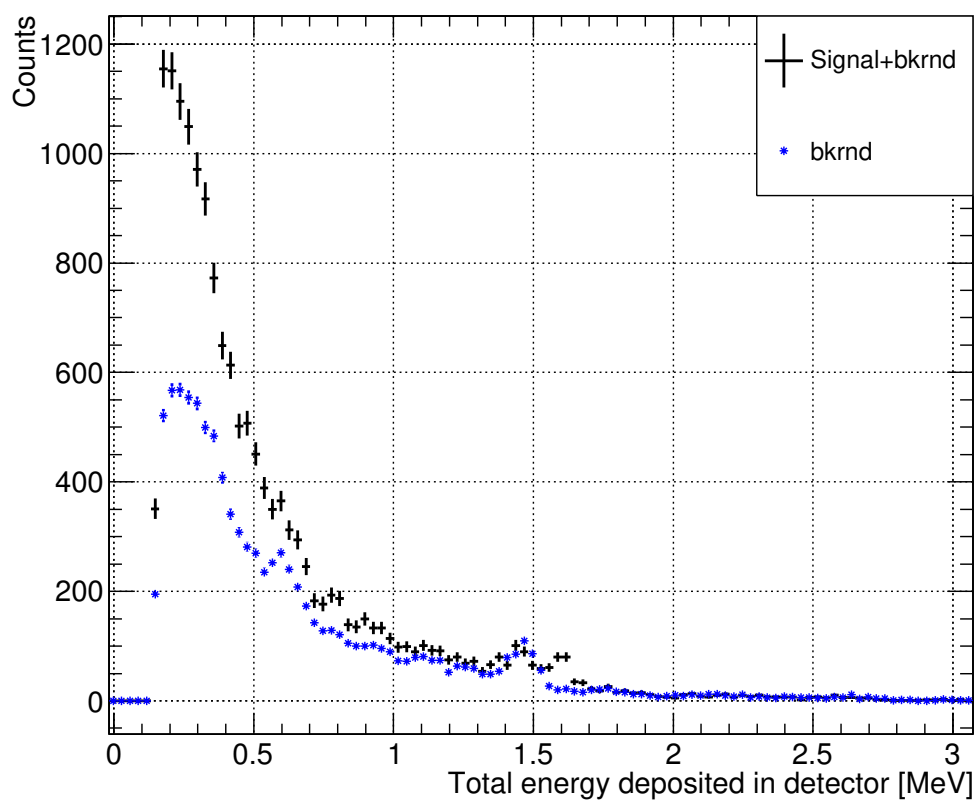

(a)

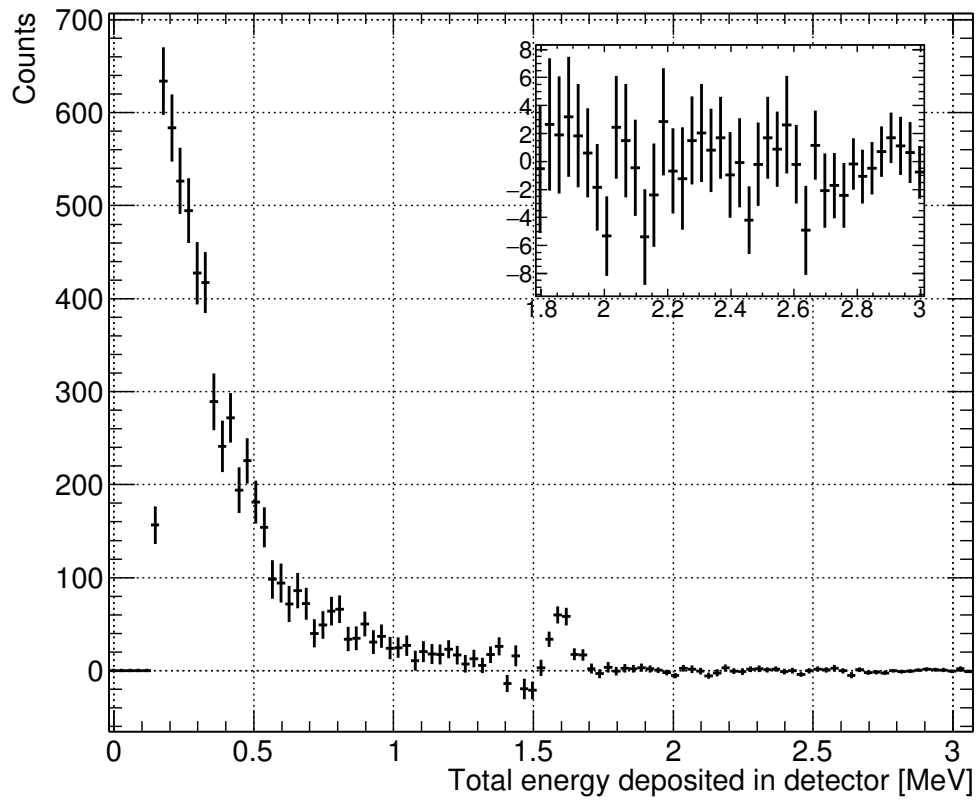

(b)

Figure 6.4: Total-energy spectrum at dwell point 0 in the Suffield experiment. a) Background +La-140 two-hit total-energy spectrum in black, and background-only spectrum in blue. b) Background-subtracted two-hit total-energy spectrum. Inset shows zoomed-in view of spectrum at high energies. Bins in both subfigures are $30 \mathrm{keV}$. 


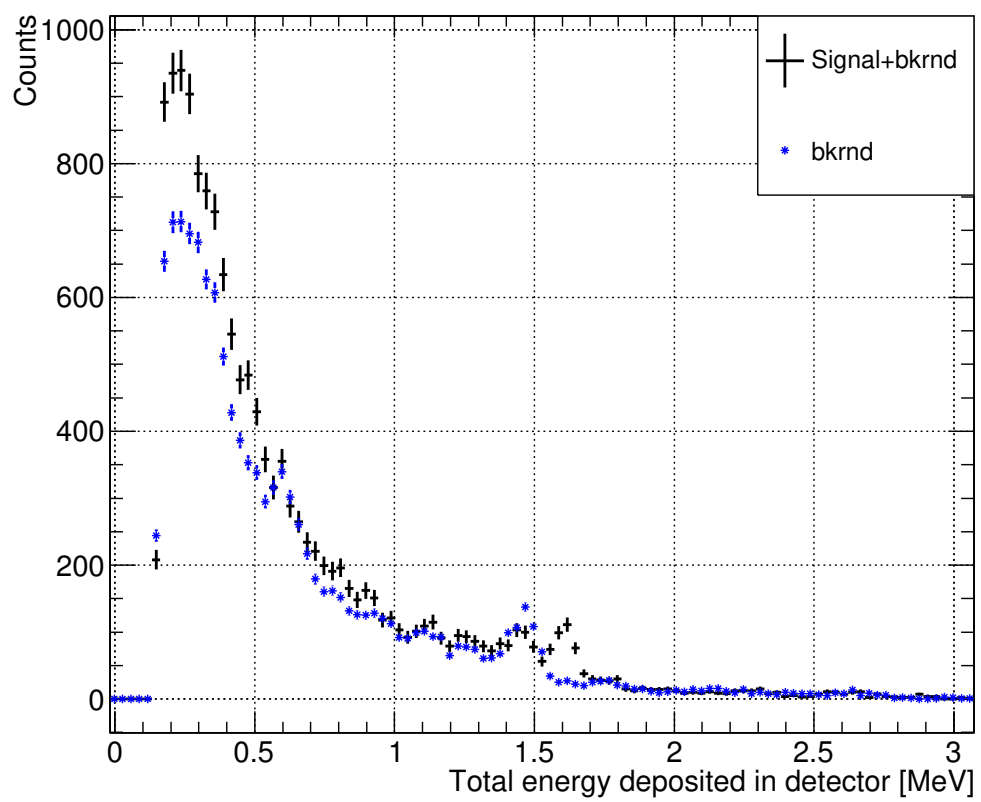

(a)

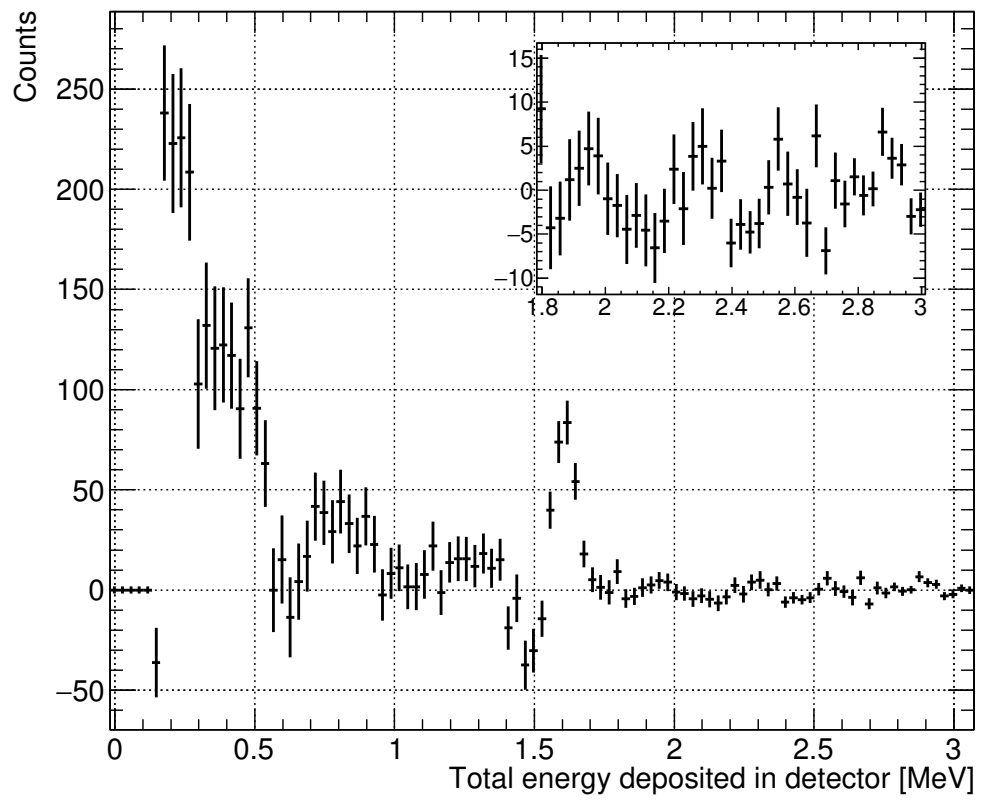

(b)

Figure 6.5: Total-energy spectrum at dwell point 1 in the Suffield experiment. a) Background +La-140 two-hit total-energy spectrum in black, and background-only spectrum in blue. b) Background-subtracted two-hit total-energy spectrum. Inset shows zoomed-in view of spectrum at high energies. Bins in both subfigures are $30 \mathrm{keV}$. 


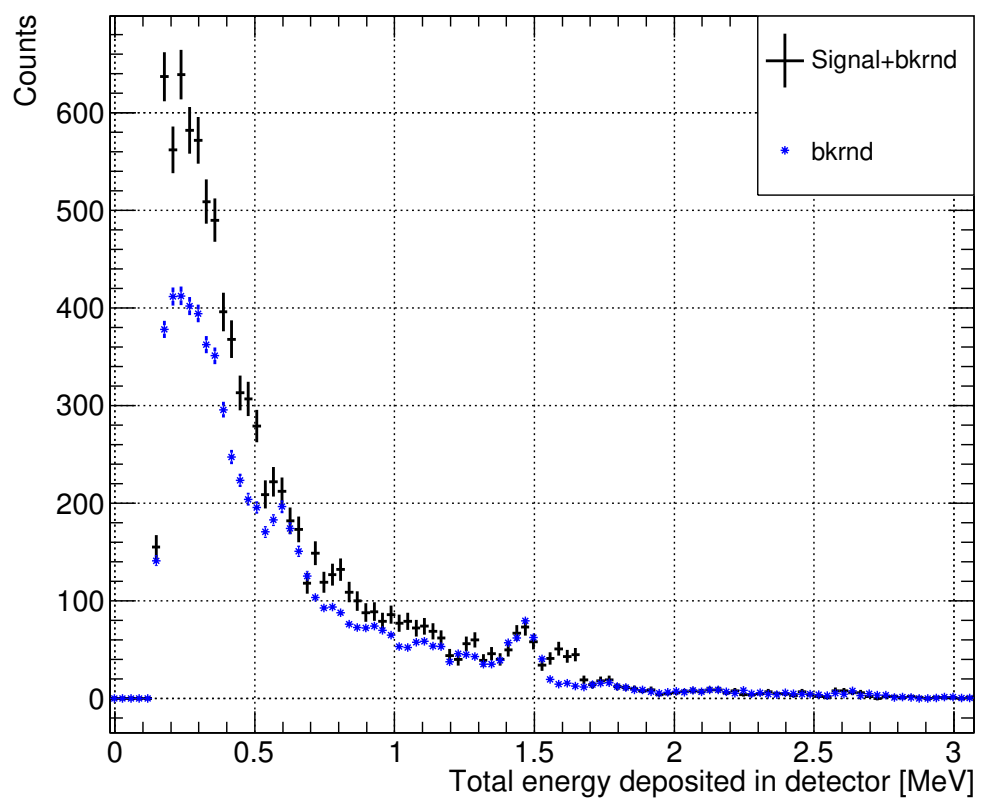

(a)

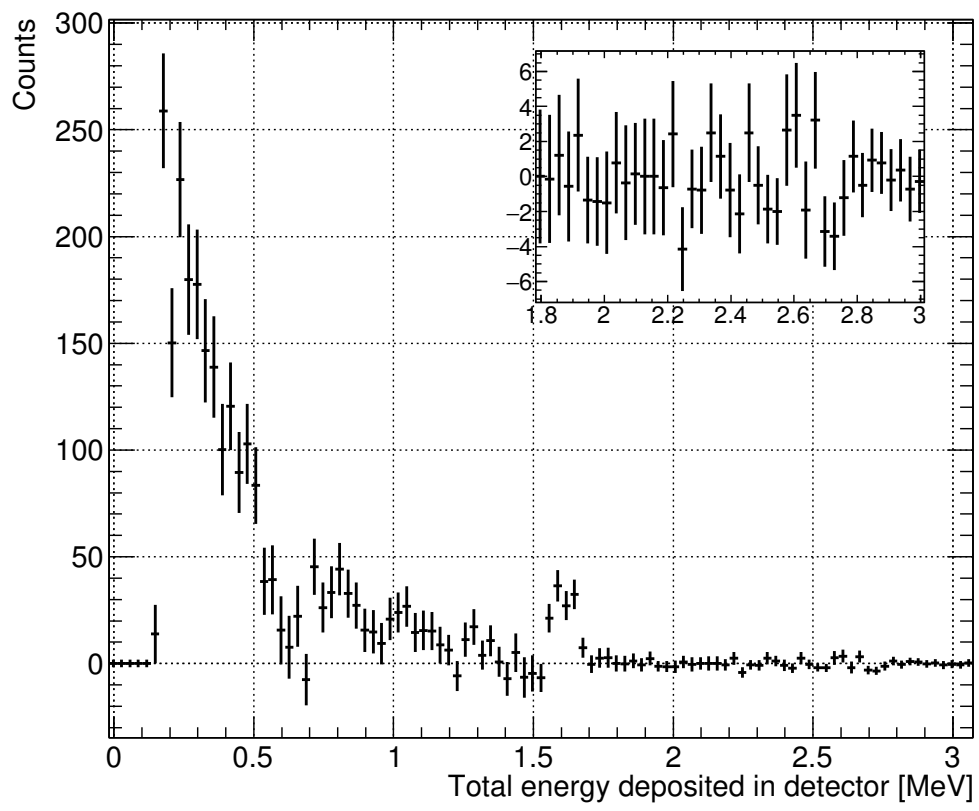

(b)

Figure 6.6: Total-energy spectrum at dwell point 2 in the Suffield experiment. a) Background +La-140 two-hit total-energy spectrum in black, and background-only spectrum in blue. b) Background-subtracted two-hit total-energy spectrum. Inset shows zoomed-in view of spectrum at high energies. Bins in both subfigures are $30 \mathrm{keV}$. 


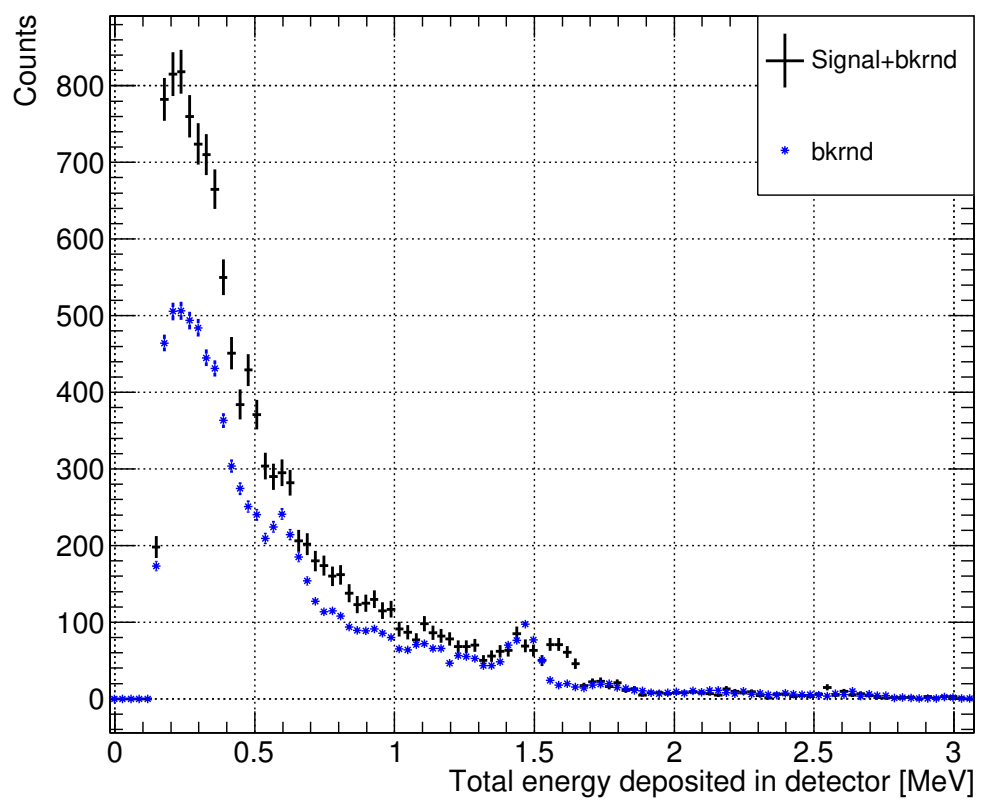

(a)

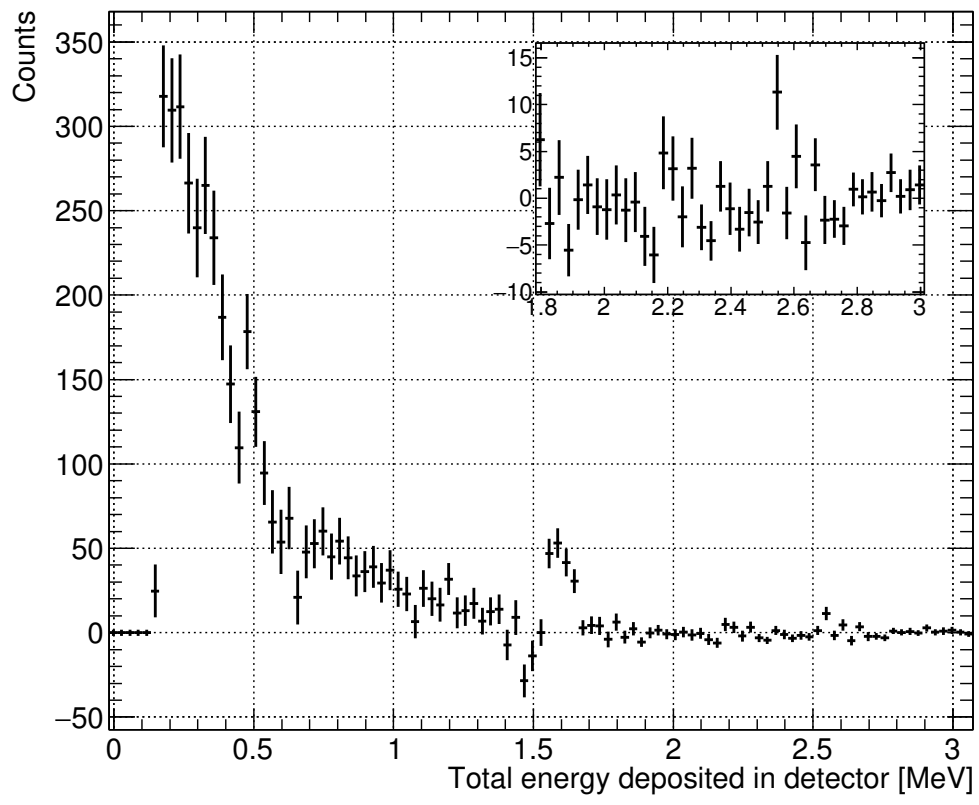

(b)

Figure 6.7: Total-energy spectrum at dwell point 3 in the Suffield experiment. a) Background +La-140 two-hit total-energy spectrum in black, and background-only spectrum in blue. b) Background-subtracted two-hit total-energy spectrum. Inset shows zoomed-in view of spectrum at high energies. Bins in both subfigures are $30 \mathrm{keV}$. 


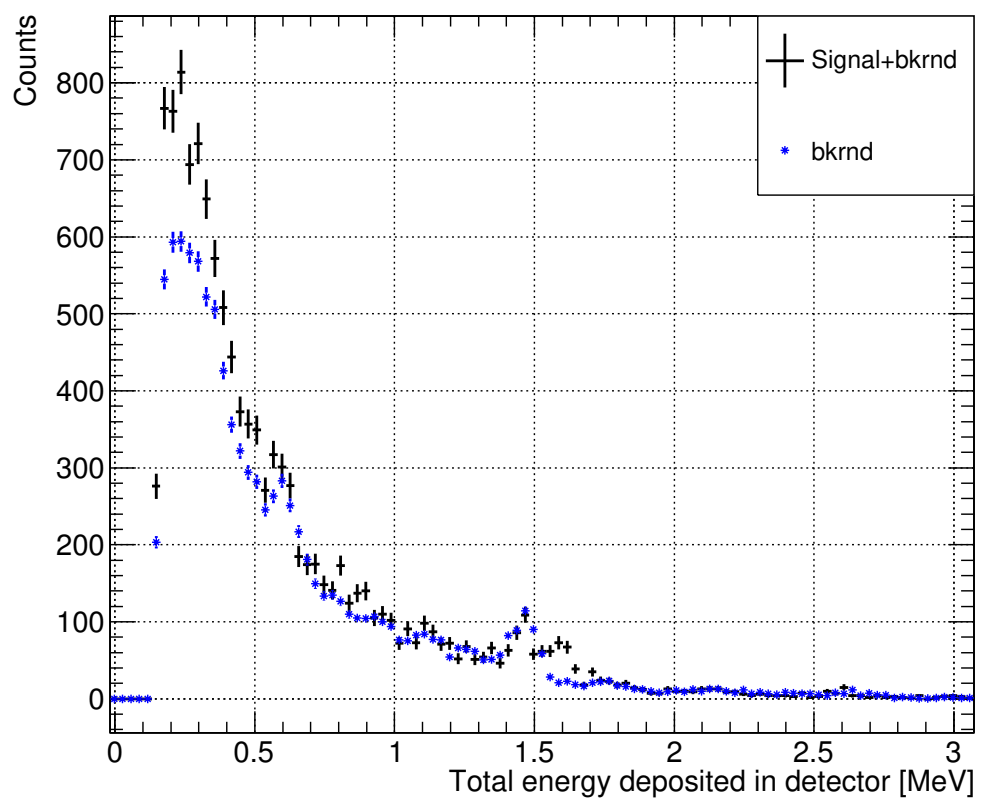

(a)

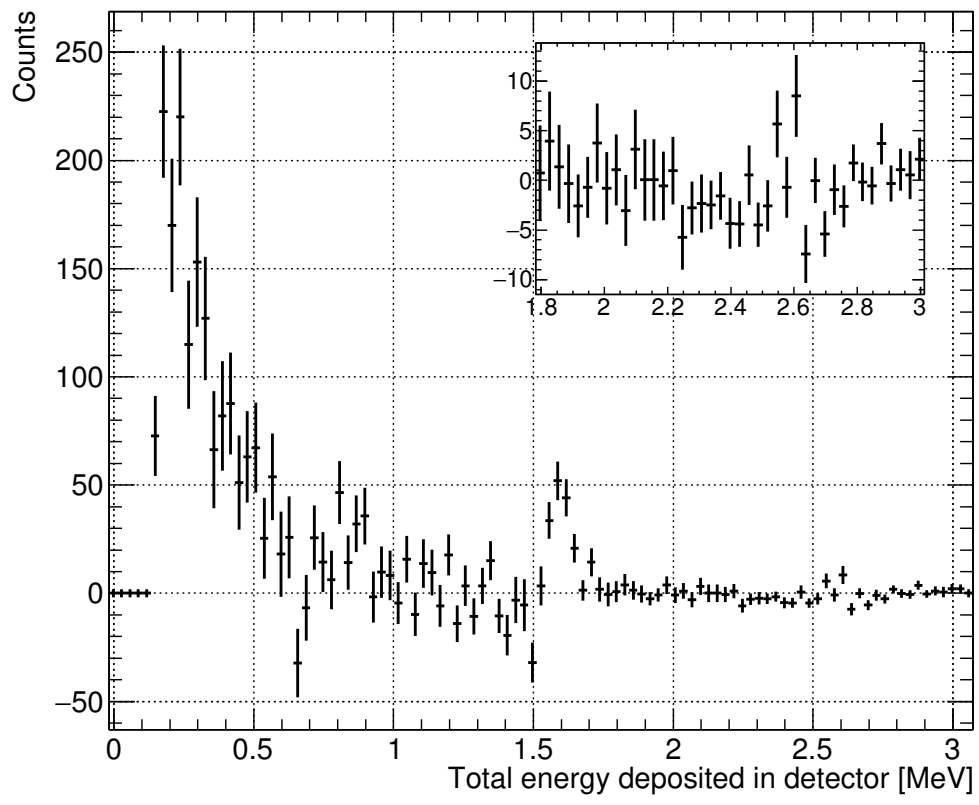

(b)

Figure 6.8: Total-energy spectrum at dwell point 4 in the Suffield experiment. a) Background +La-140 two-hit total-energy spectrum in black, and background-only spectrum in blue. b) Background-subtracted two-hit total-energy spectrum. Inset shows zoomed-in view of spectrum at high energies. Bins in both subfigures are $30 \mathrm{keV}$. 


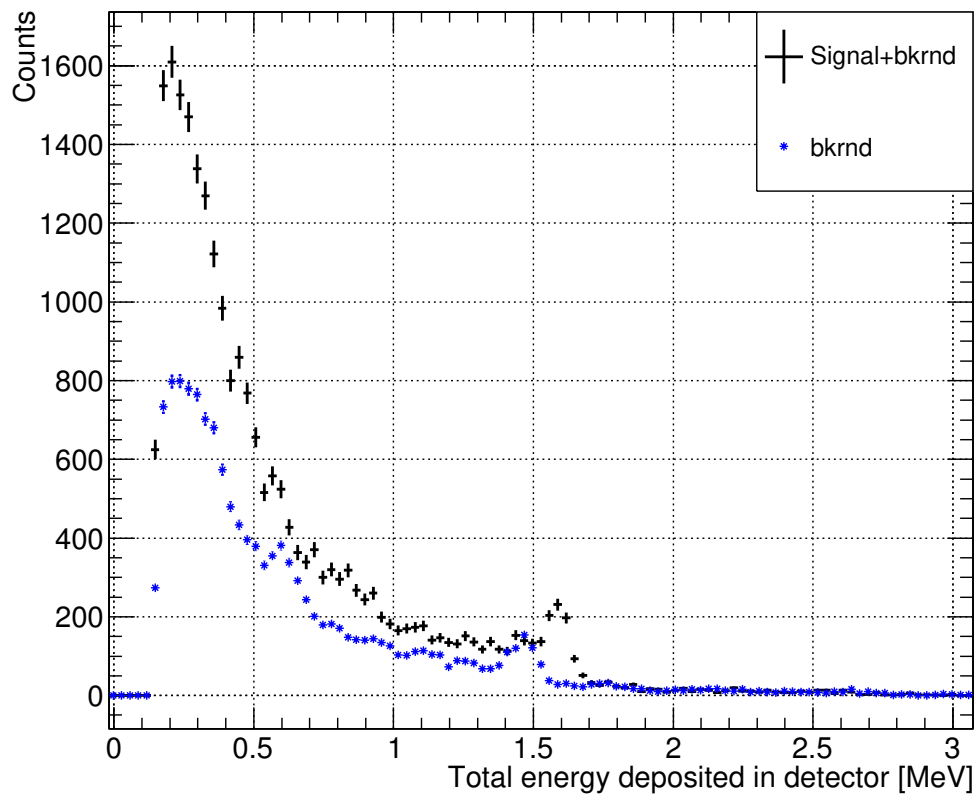

(a)

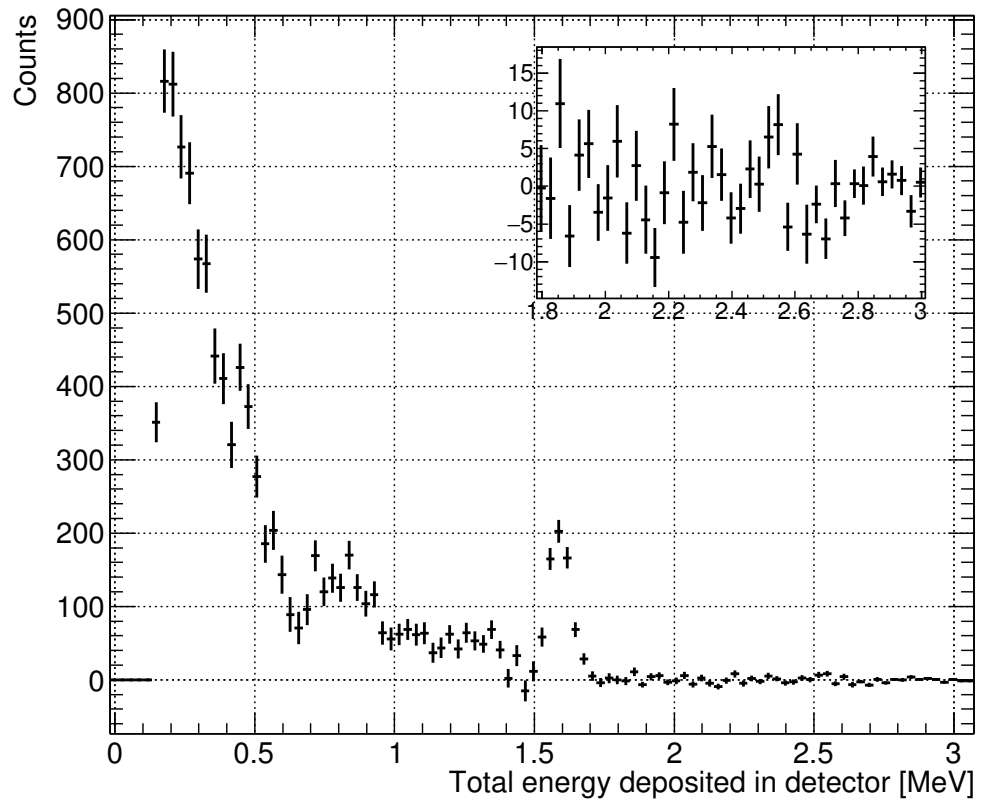

(b)

Figure 6.9: Total-energy spectrum at dwell point 5 in the Suffield experiment. a) Background +La-140 two-hit total-energy spectrum in black, and background-only spectrum in blue. b) Background-subtracted two-hit total-energy spectrum. Inset shows zoomed-in view of spectrum at high energies. Bins in both subfigures are $30 \mathrm{keV}$. 


\section{Event selection}

Table 6.2 lists the 38 gamma-ray emissions of La-140, where the four most prominent emissions are indicated by bold text. The $1.596 \mathrm{MeV}$ gamma-ray emission dominates with 95.4 photons emitted per 100 decays. Due to its relative abundance, the $1.596 \mathrm{MeV}$ emission is selected for Compton gamma imaging. The contribution of this emission to the data is evident in figures 6.4 to 6.9.

The selection criteria for the $1.596 \mathrm{MeV}$ gamma-ray emission are chosen in accordance with the principles outlined in section 4.2, and are presented in table 6.3.

Table 6.4 shows the number of events passing the event-selection criteria given in table 6.3 at each dwell point. In total, there are 1049 events accepted across all six dwell points. 
Table 6.2: Gamma-ray emissions of La-140. Bold text indicates the four most prominent emissions. This table was generated from the data provided in reference 78.

\begin{tabular}{l|l} 
Energy [keV] & Photons per 100 decays \\
\hline 24.595 & 0.000689 \\
64.129 & 0.014 \\
68.923 & 0.077 \\
109.417 & 0.217 \\
131.121 & 0.47 \\
173.546 & 0.126 \\
241.959 & 0.436 \\
266.554 & 0.492 \\
307.08 & 0.022 \\
$\mathbf{3 2 8 . 7 6 1}$ & $\mathbf{2 0 . 8}$ \\
397.674 & 0.075 \\
432.513 & 3 \\
438.178 & 0.017 \\
444.57 & 0.003 \\
$\mathbf{4 8 7 . 0 2 2}$ & $\mathbf{4 6 . 1}$ \\
618.12 & 0.041 \\
751.653 & 4.39 \\
$\mathbf{8 1 5 . 7 8 4}$ & $\mathbf{2 3 . 7 2}$ \\
867.839 & 5.58 \\
919.533 & 2.73 \\
925.198 & 7.04 \\
950.988 & 0.531 \\
992.64 & 0.01 \\
1045.02 & 0.02 \\
1097.58 & 0.023 \\
1303.34 & 0.045 \\
1404.66 & 0.062 \\
$\mathbf{1 5 9 6 . 2 0 3}$ & $\mathbf{9 5 . 4}$ \\
1877.33 & 0.041 \\
1924.5 & 0.011 \\
2083.219 & 0.036 \\
2347.847 & 0.845 \\
2464.031 & 0.0097 \\
2521.39 & 3.41 \\
2547.18 & 0.102 \\
2899.53 & 0.066 \\
3118.49 & 0.026 \\
3319.52 & 0.0039 \\
&
\end{tabular}


Table 6.3: Energy cuts used with the 3x3-module SCoTSS detector in event selection for the $1.596 \mathrm{MeV}$ emission of La-140.

\begin{tabular}{l|l} 
Selection criteria & Energy requirements \\
\hline Preselection and noise rejection & $E_{1}>15 \mathrm{keV}$ and $E_{2}>25 \mathrm{keV}$ \\
& and $E_{1}+E_{2}>150 \mathrm{keV}$ \\
Photopeak selection & $1.52 \mathrm{MeV}<E_{1}+E_{2}<1.68 \mathrm{MeV}$ \\
Back-scatter rejection & $E_{1}<0.18 \mathrm{MeV}$ or $0.30<E_{1} \mathrm{MeV}$ \\
Geometric selection & $E_{1}<0.80 \mathrm{MeV}$
\end{tabular}

Table 6.4: Number of events passing the selection criteria at each dwell point in the Suffield experiment.

\begin{tabular}{c|c} 
Dwell point & Events passing cuts \\
\hline 0 & 136 \\
1 & 200 \\
2 & 92 \\
3 & 115 \\
4 & 119 \\
5 & 387
\end{tabular}

\section{Individual dwell-point Compton gamma images}

Figure 6.10a shows an overlay of the gamma image on an optical image acquired during the experimental measurement at dwell point 0 , where the contours of the gamma image shown are as they existed during the experimental trial and do not include background subtraction. To co-register the gamma image to the optical image, the Compton cone information has been adjusted to agree with the coordinate system of the optical photograph. Figure 6.10b shows a gamma image created in post-processing from the data acquired at dwell point 0 that includes backgroundsubtraction. Figures 6.11 to 6.15 show similar sets of images for dwell points 0 to 5. The approximation of the source as sitting at the horizon (i.e. a vertical angle of $0^{\circ}$ ) in the FOV of the detector can be seen to be a reasonable assumption, as the gamma images reach peak intensity very near the horizon and the experimental zone extends near the horizon in the optical images. The gamma images also reflect the sparsity of the data, as some of the Compton rings of individual events are visible for some dwell points (e.g. dwell point 2 in figure 6.12b). These point-of-view gamma images are not directly used for the tomographic reconstruction methodologies that 
are presented below in this chapter. However, they may be used in-situ to localize the radioactive source in the field of view of the detector and to guide the operator.

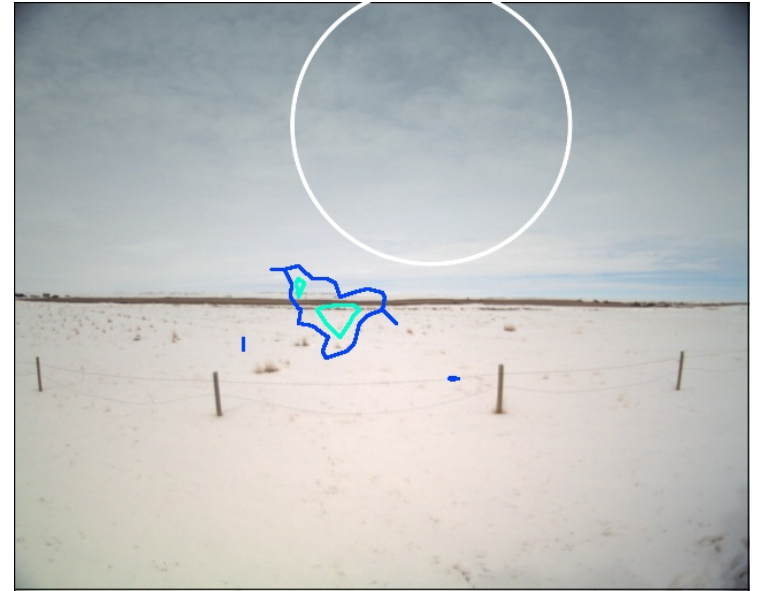

(a)

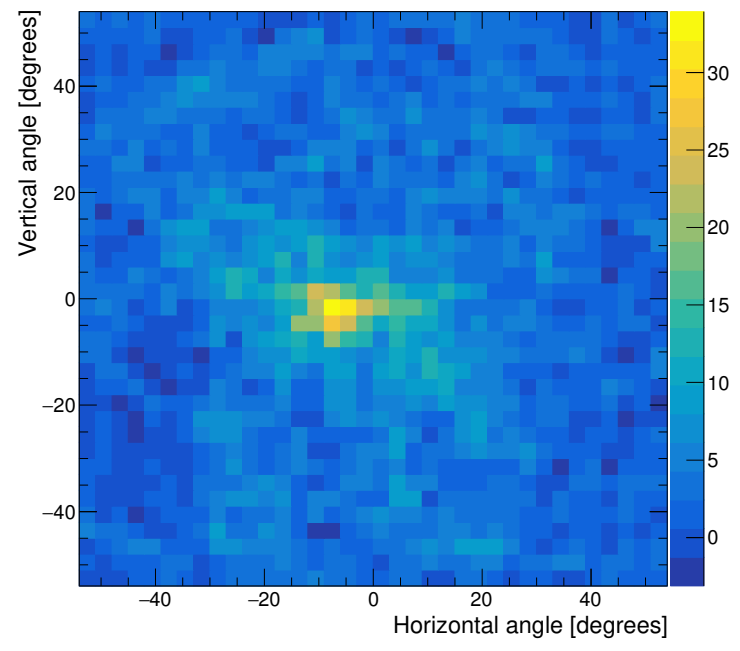

(b)

Figure 6.10: Field-of-view gamma image at dwell point 0 in the Suffield experiment. a) Field-of-view image overlaid on optical image, as acquired during the experiment. b) Background-subtracted field-of-view image generated in post-processing. 


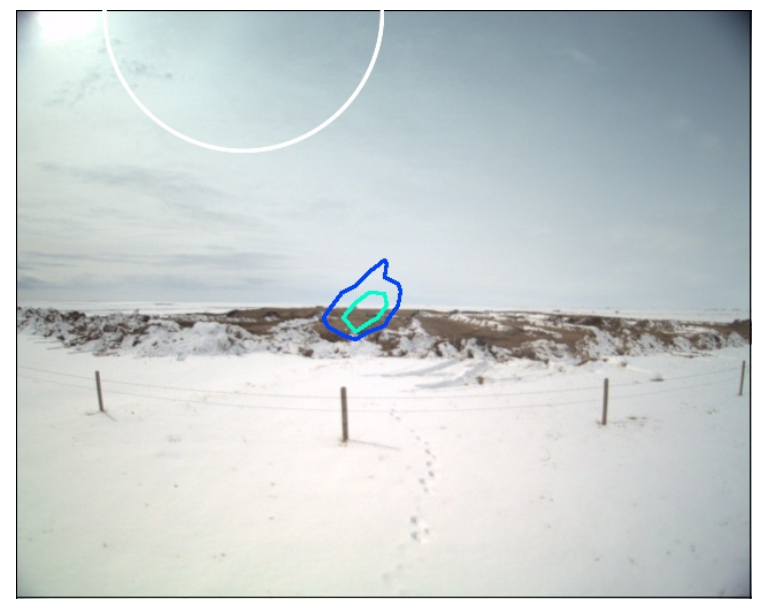

(a)

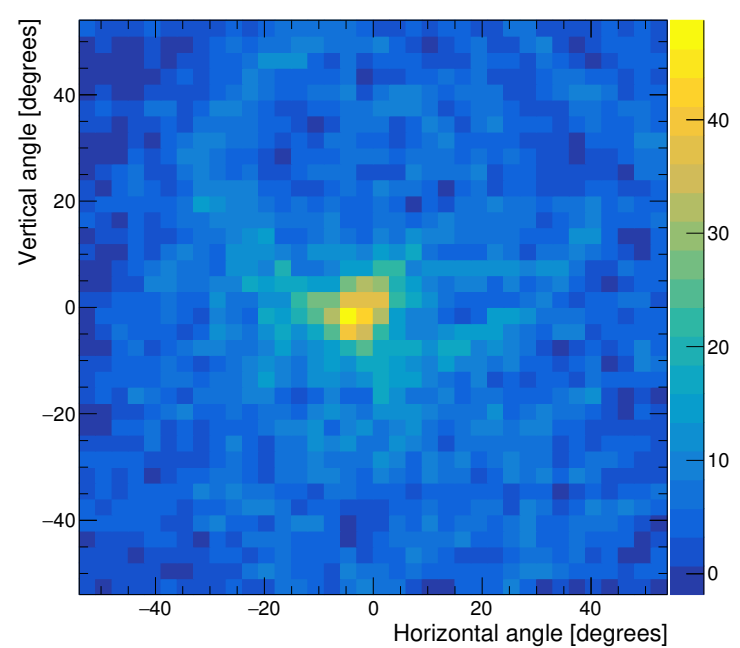

(b)

Figure 6.11: Field-of-view gamma image at dwell point 1 in the Suffield experiment. a) Field-of-view image overlaid on optical image, as acquired during the experiment. b) Background-subtracted field-of-view image generated in post-processing.

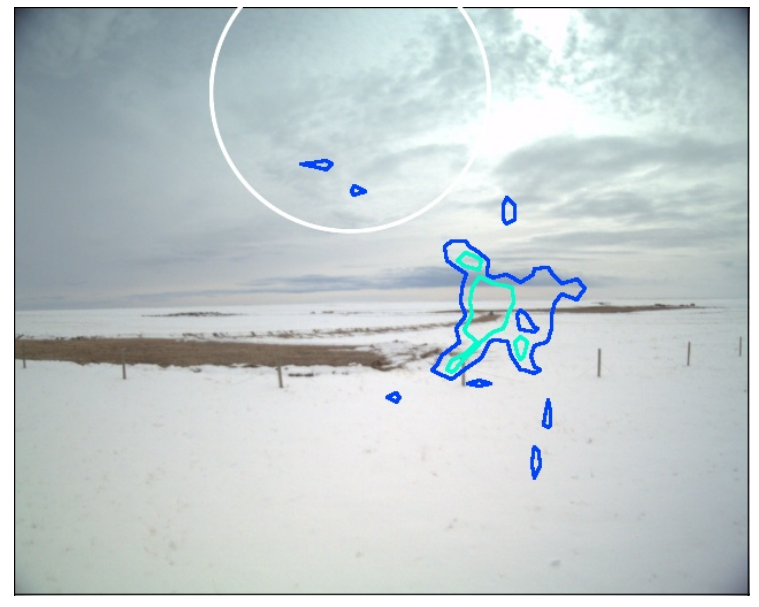

(a)

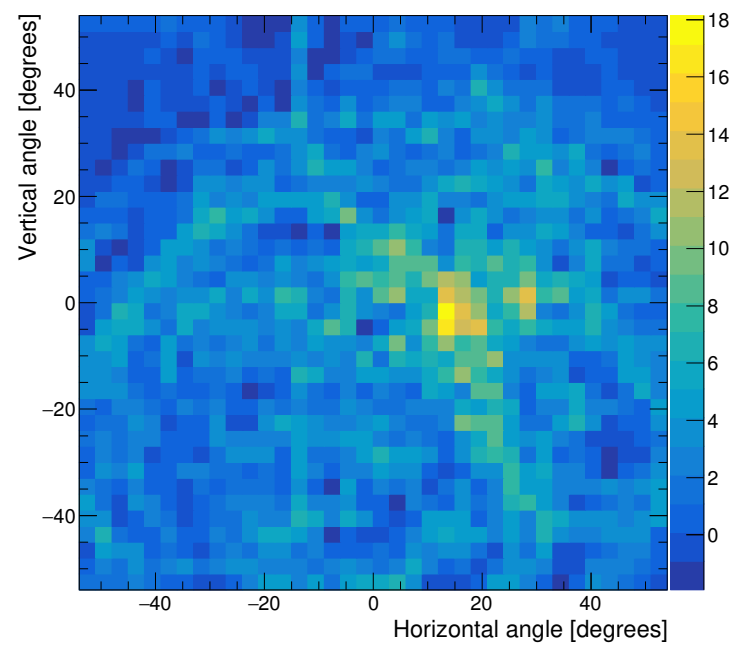

(b)

Figure 6.12: Field-of-view gamma image at dwell point 2 in the Suffield experiment. a) Field-of-view image overlaid on optical image, as acquired during the experiment. b) Background-subtracted field-of-view image generated in post-processing. 


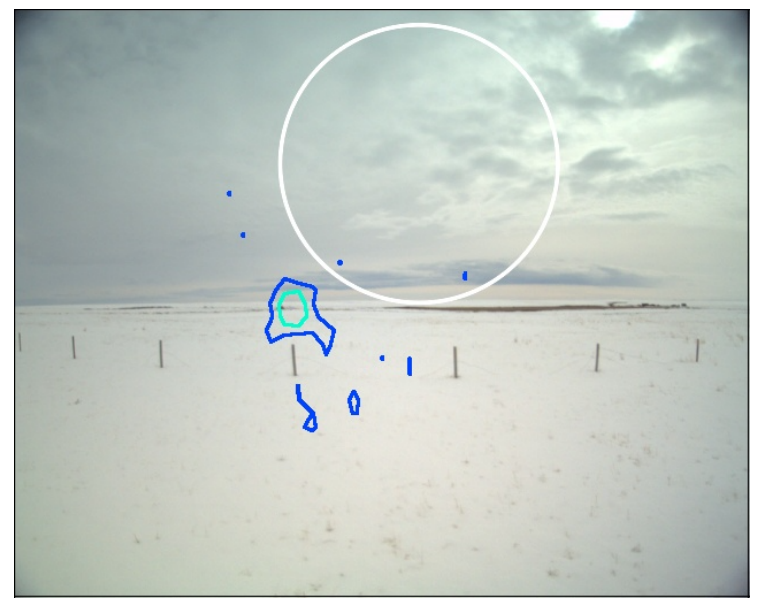

(a)

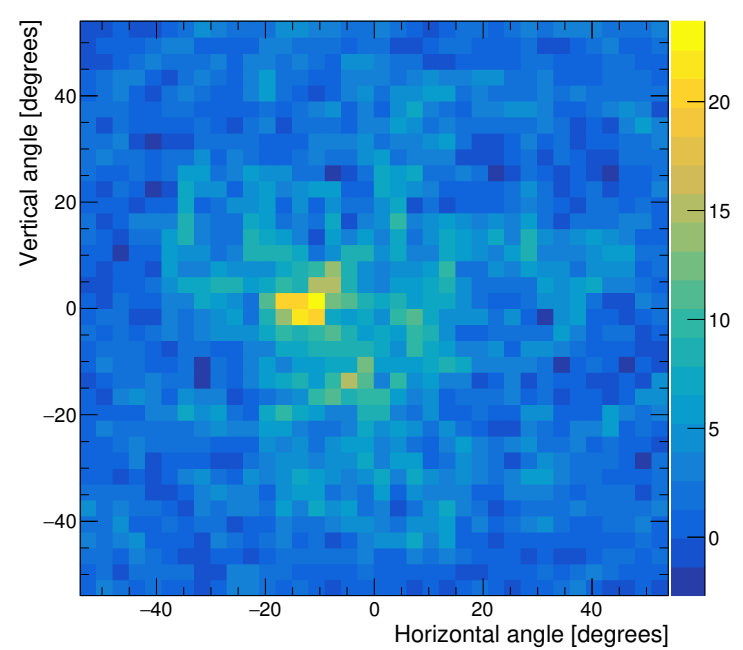

(b)

Figure 6.13: Field-of-view gamma image at dwell point 3 in the Suffield experiment. a) Field-of-view image overlaid on optical image, as acquired during the experiment. b) Background-subtracted field-of-view image generated in post-processing.

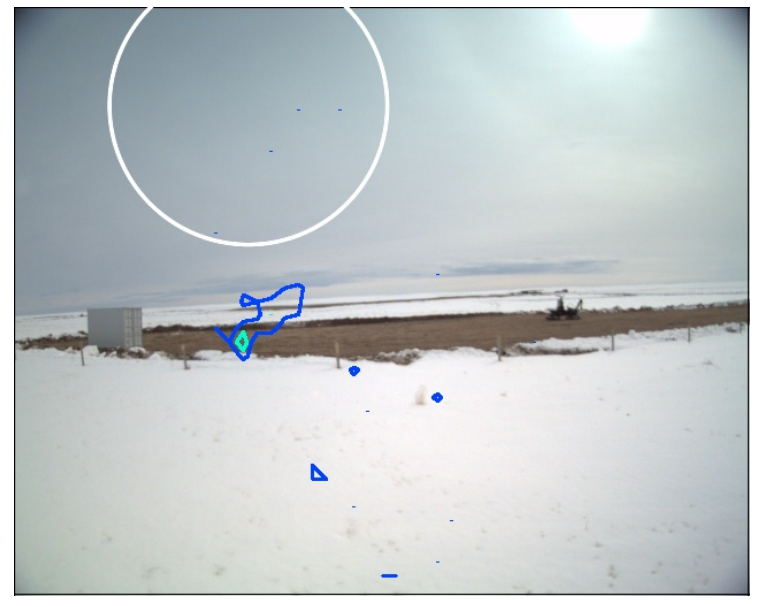

(a)

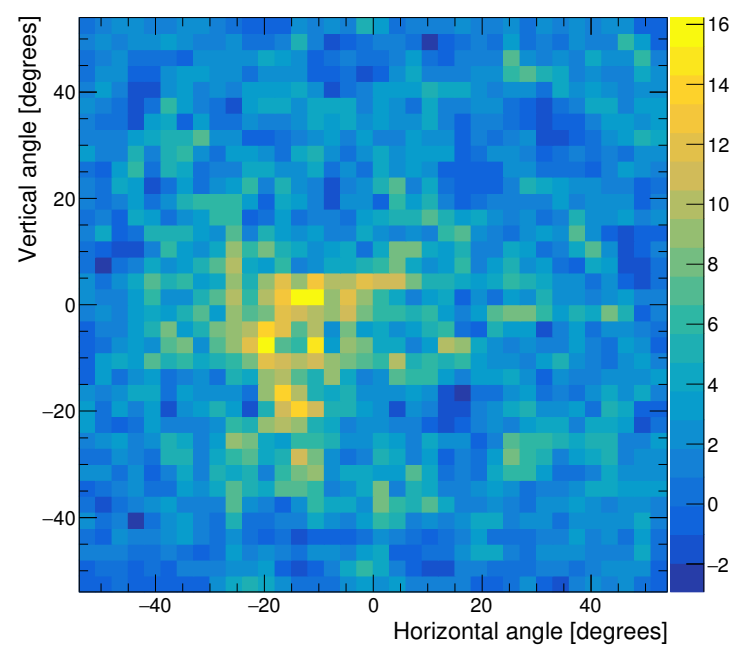

(b)

Figure 6.14: Field-of-view gamma image at dwell point 4 in the Suffield experiment. a) Field-of-view image overlaid on optical image, as acquired during the experiment. b) Background-subtracted field-of-view image generated in post-processing. 


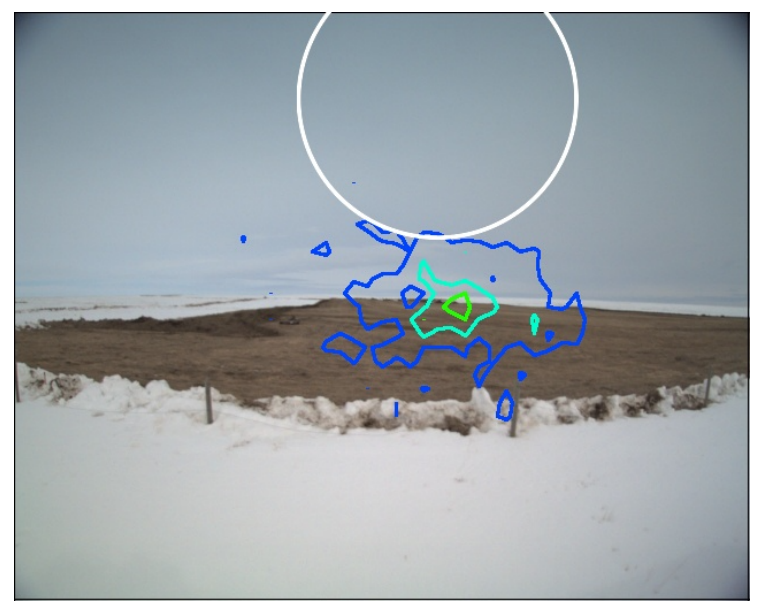

(a)

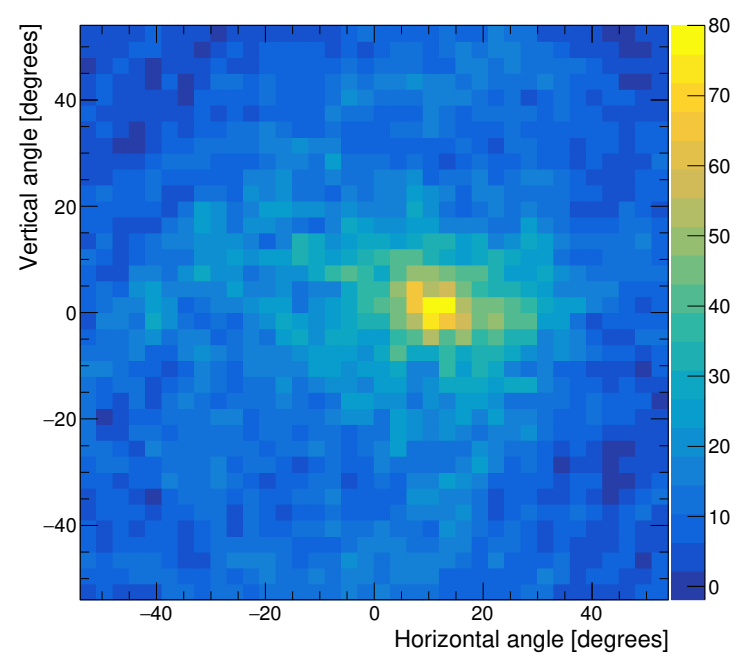

(b)

Figure 6.15: Field-of-view gamma image at dwell point 5 in the Suffield experiment. a) Field-of-view image overlaid on optical image, as acquired during the experiment. b) Background-subtracted field-of-view image generated in post-processing. 


\subsubsection{Estimating the position and orientation of the $3 \times 3$ - module SCoTSS detector}

The on-board GNSS equipment recorded second-by-second position information during the transit periods in between each of the dwell points, as well as throughout the entire data-acquisition period at each dwell point. This spatial information was used to estimate both the position and the orientation of the 3x3-module SCoTSS detector at each dwell point.

The position of the detector at each dwell point was estimated by averaging the second-by-second GNSS position information over the duration during which the detector was stationary and acquiring gamma-ray data. The resulting average positions were in units of latitude and longitude, which were subsequently converted to UTM easting and northing coordinates. To make the coordinates more convenient to work with, an arbitrary offset was subtracted from each of the easting and northing axes; this resulted in the coordinate system presented in figure 6.1. Table 6.5 lists the average coordinates of each dwell point, along with the standard deviation in GNSS measurements. In addition to the statistical fluctuations on the GNSS coordinates provided in table 6.5, an additional $3 \mathrm{~m}$ systematic uncertainty on the GNSS coordinates exists due to the limited accuracy of the GNSS equipment.

The 3x3-module SCoTSS detector used to acquire data was not equipped with any instrumentation to record its orientation. To estimate the detector's orientation at each dwell point, three assumptions were made:

1. the detector faced left with respect to the direction of propagation of the BV206 as viewed from behind, exactly perpendicular to the direction of motion;

2. the BV206 drove parallel to the border of the restricted-access zone on all sides of the perimeter, so that the axes of both carriages of the vehicle were collinear; and

3. the central plane of the detector remained parallel with the surface of the ground (i.e. there were no significant pitch or roll angles in the detector's orientation).

Each of these assumptions were reasonably satisfied under the experimental conditions. The heading of the BV206 as it drove into position at each dwell point was first 
estimated. This was accomplished by taking a GNSS coordinate near the beginning of the vehicle's trajectory along one side of the restricted-access zone and the final position of the vehicle, and fitting a line between them. The angle of the resulting line relative to the easting axis was extracted. The 3x3-module SCoTSS detector was assumed to be oriented exactly $90^{\circ}$ counter-clockwise to the BV206 heading angle. An uncertainty of $3 \mathrm{~m}$ was assumed on each GNSS coordinate, and this uncertainty was propagated through to the estimate of the yaw angle component of the orientation of the detector. The resulting yaw angle estimates are indicated by red arrows in figure 6.1, and table 6.5 lists each yaw angle along with the estimated uncertainty. The orientation angle of the detector is reported relative to the easting axis and in the half-bounded interval $\left[0^{\circ}, 360^{\circ}\right)$.

Table 6.5: Detector coordinates and orientations for the experimental dwell-point positions. Coordinates are presented in UTM easting and northing with an arbitrary offset applied. A systematic uncertainty of $3 \mathrm{~m}$ exists on the dwell point coordinates in addition to the statistical errors provided in the table. The orientations are measured with respect to the easting axis.

\begin{tabular}{c|c|c|c} 
Dwell point & Easting $(\mathrm{m})$ & Northing $(\mathrm{m})$ & Detector yaw angle $(\mathrm{deg})$ \\
\hline 0 & $435.0 \pm 0.1$ & $98.6 \pm 0.1$ & $120.4 \pm 1.9$ \\
1 & $586.0 \pm 0.2$ & $459.5 \pm 0.3$ & $210.5 \pm 1.2$ \\
2 & $308.2 \pm 0.1$ & $623.5 \pm 0.1$ & $299.7 \pm 1.8$ \\
3 & $202.1 \pm 0.2$ & $563.6 \pm 0.1$ & $299.6 \pm 2.1$ \\
4 & $119.6 \pm 0.1$ & $517.0 \pm 0.1$ & $299.6 \pm 2.7$ \\
5 & $121.3 \pm 0.1$ & $237.9 \pm 0.2$ & $30.8 \pm 1.3$
\end{tabular}

\subsubsection{Monte Carlo simulation of detector response}

\section{Simulation geometry}

The point-model response and the response templates generated for spatial unfolding employed the same simulated environment. As detailed in section 4.6, only air was included in the simulated environment. The simulated air had a density of $1.2116 \times$ $10^{-3} \mathrm{~g} / \mathrm{cm}^{3}$, consistent with dry air at a temperature of $0{ }^{\circ} \mathrm{C}$ and at a pressure of $95 \mathrm{kPa}$.

The interior components of the simulated representation of the $3 \times 3$-module SCoTSS detector were as presented in figure 4.9, and an additional carbon fibre and 
polystyrene enclosure was added to approximate the enclosure of the detector that was used during the experiment. Unlike the detector used for the work presented in chapter 5, the detector used during the Suffield experiment did not have five-sided carbon fibre covers on each detector plane. Figure 6.16 shows a view of the simulated detector as it existed during the Suffield experiment, where a cross section along an oblique plane has been generated to provide a view inside the enclosure. Yellow material represents carbon fibre, dark red material represents polystyrene, magenta represents the aluminum module cases and green represents the $\mathrm{CsI}(\mathrm{Tl})$ crystals (not visible at the scale of the image are the simulated SiPMs).

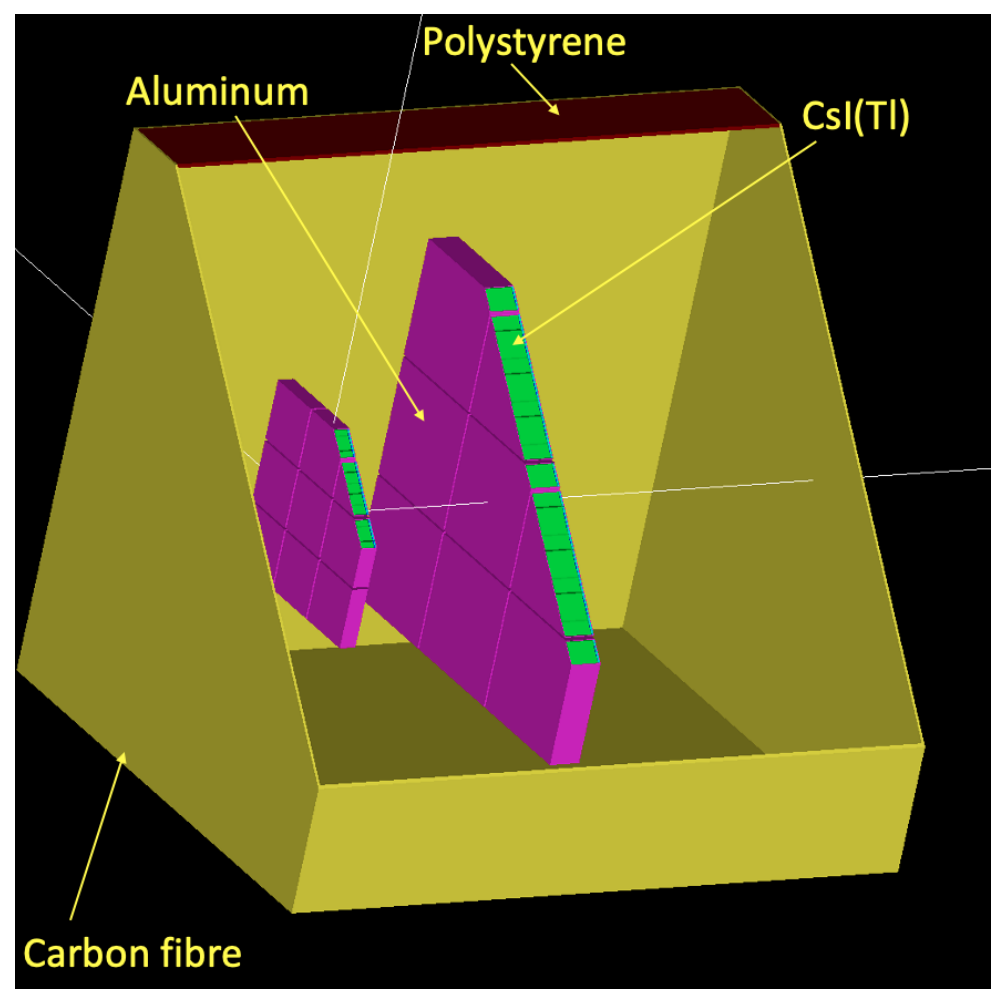

Figure 6.16: View of the simulated 3x3-module SCoTSS detector geometry used for the Suffield experiment. An oblique cross section has been cut to allow a view of the interior. The yellow material represents carbon fibre, the dark red material represents polystyrene, the green material represents $C s I(T l)$, and the magenta material represents aluminum.

To reflect the resolution of each channel as it existed in the experimental configuration, the empirical energy-smearing function used in the MC simulations and provided in equation 4.9 was adjusted using calibration data measured at $662 \mathrm{keV}$ 
as per the procedure described in section 4.6. Figure 6.17 shows the experimentallymeasured FWHM resolutions at $662 \mathrm{keV}$ obtained from calibration with a Cs-137 check source, plotted for each detector plane as a function of the Cartesian indices of each channel. Each map has been plotted on a common colour scale, and white indicates a dead channel. The detector that was used was an active research instrument and was not optimized to commercial standards. Dead channels may result from disconnected cables, noisy channels, or dead pre-amplifier inputs, and no attempt was made to repair dead channels prior to the experiment. The energy resolutions shown in figure 6.17 were derived from fits to energy spectra collected in situ under rapidly changing environmental conditions and underestimate the achievable detector performance. 


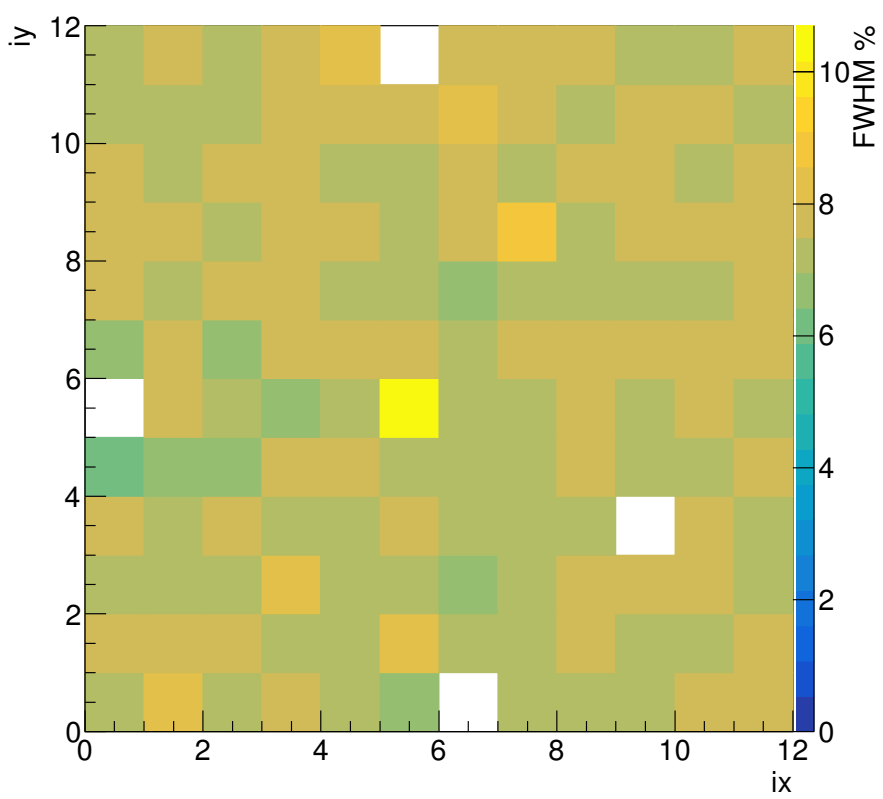

(a)

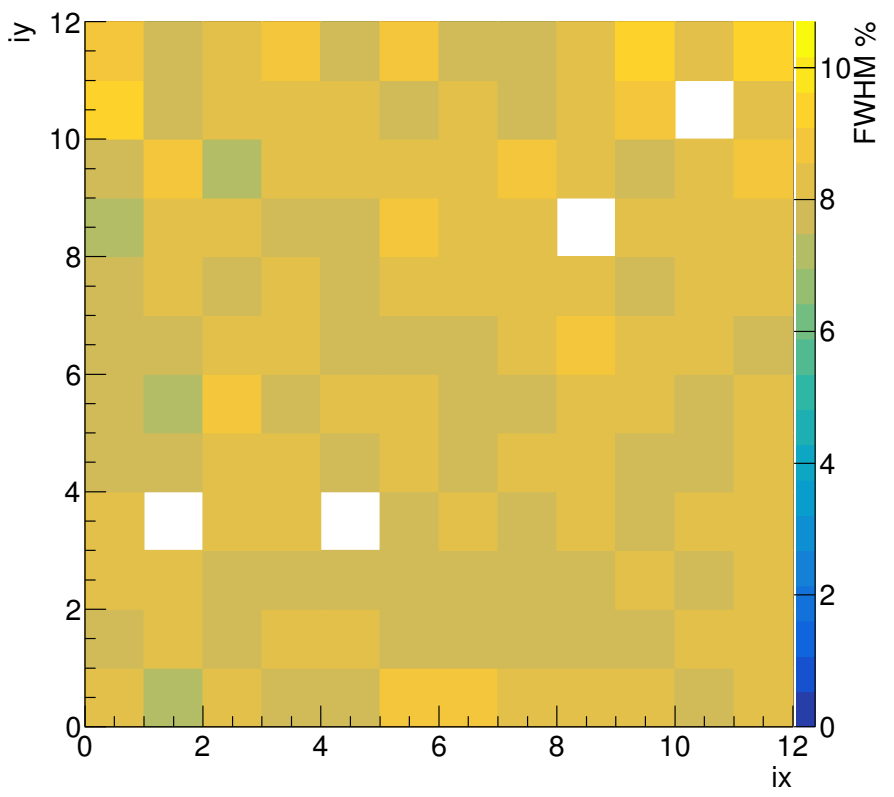

(b)

Figure 6.17: Experimentally-measured FWHM used to apply custom channel-bychannel energy smearing in simulations of the Suffield experiment. a) Scatter-plane channels. b) Absorber-plane channels. Each subfigure shows a map as a function of the Cartesian indices of each channel, plotted on a common colour scale where white indicates a dead channel. 


\section{Comparison of MC simulation to real data}

Figure 6.18 shows a comparison of experimental Compton imaging measurements to synthetic La-140 data made by MC simulation. Because no independent estimate of the true La-140 distribution is available, the synthetic dataset was generated using for the source distribution the distribution unfolded from this measurement, which will be presented in section 6.2.3. Figures $6.18 \mathrm{a}$ and $6.18 \mathrm{~b}$ show the distribution of Compton cone polar angles and Compton scattering angles, respectively, and figures $6.18 \mathrm{c}$ to 6.18e show the energy depositions in the scatter plane, in the absorber plane, and in the full detector for all two-hit events. Figures 6.19 to 6.23 show the same results for dwell points 1 to 5 . The agreement between the structure of the synthetic data and the experimental data suggests that there is not a significant aspect of the physics that has been omitted in modelling the gamma radiation that reached the detector. 


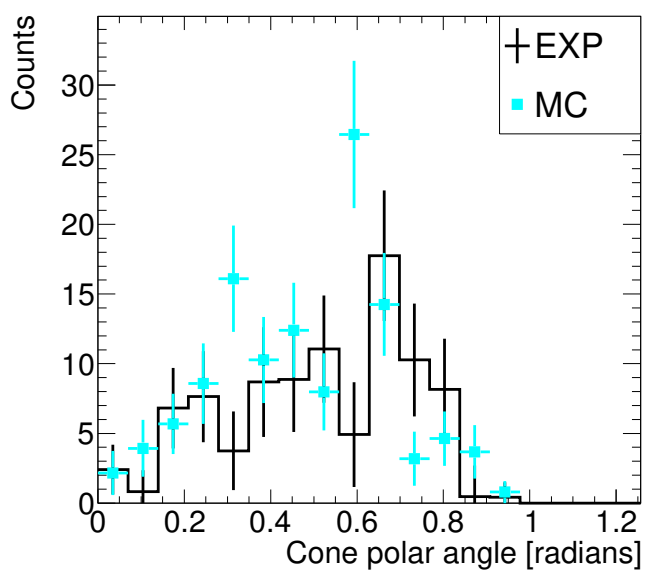

(a)

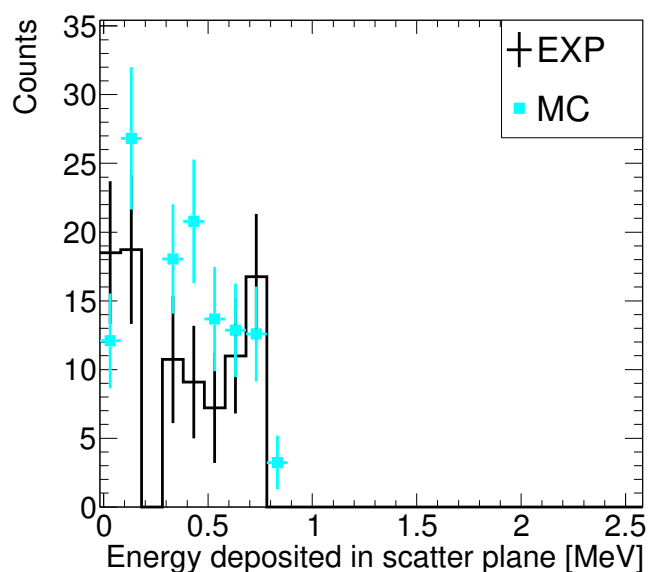

(c)

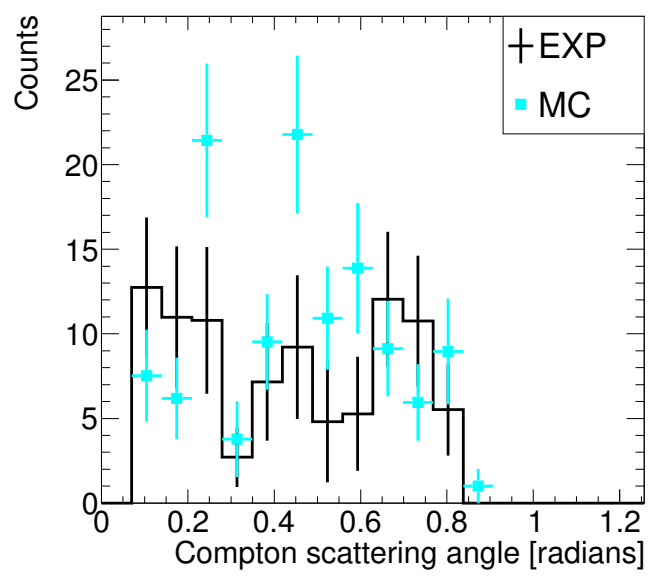

(b)

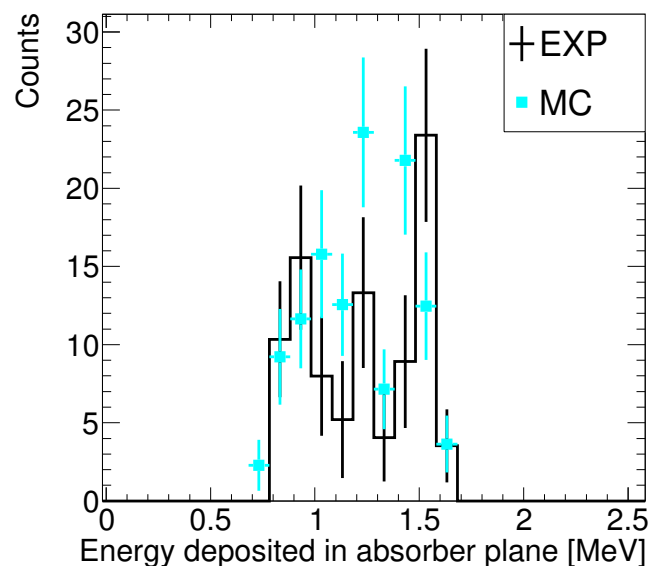

(d)

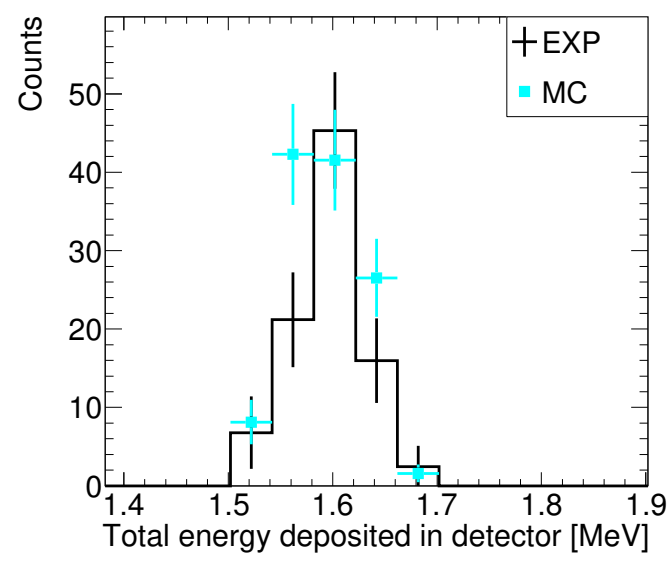

(e)

Figure 6.18: Simulated and experimental extended-source Compton imaging measurements at dwell point 0 after energy cuts. a) Compton cone polar angle. b) Compton scattering angle. c) Scatter-plane energies. d) Absorber-plane energies. e) Totalenergy depositions. 


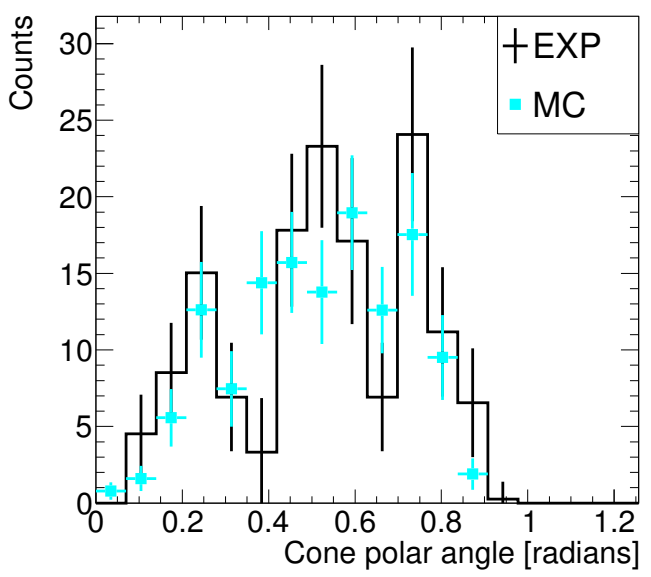

(a)

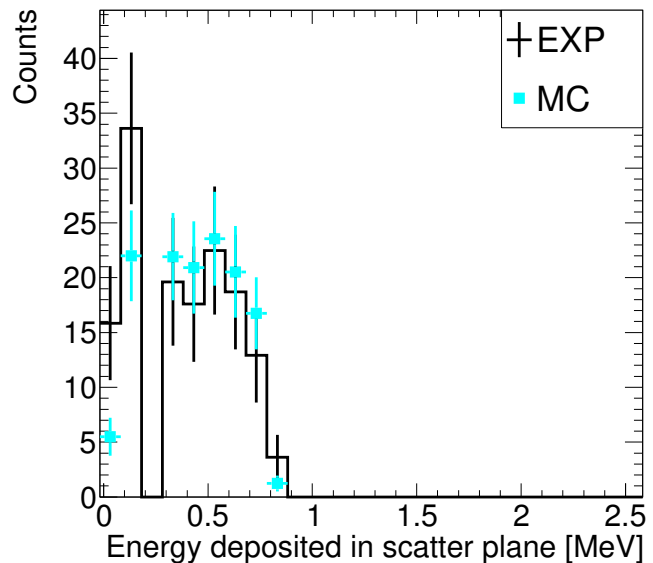

(c)

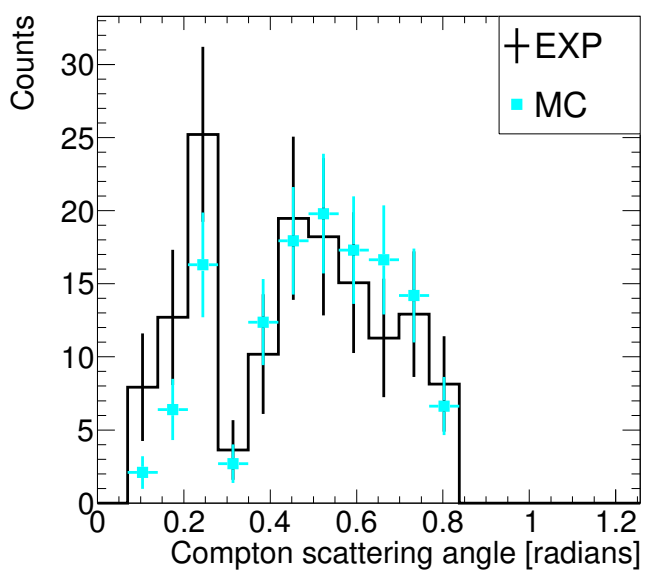

(b)

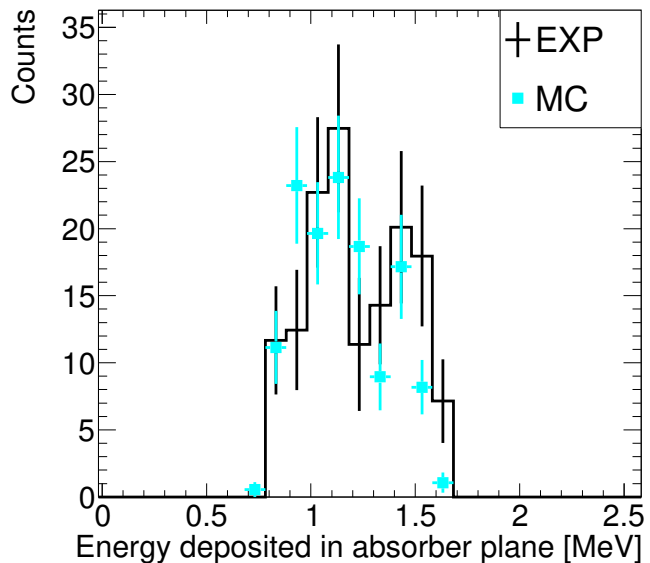

(d)

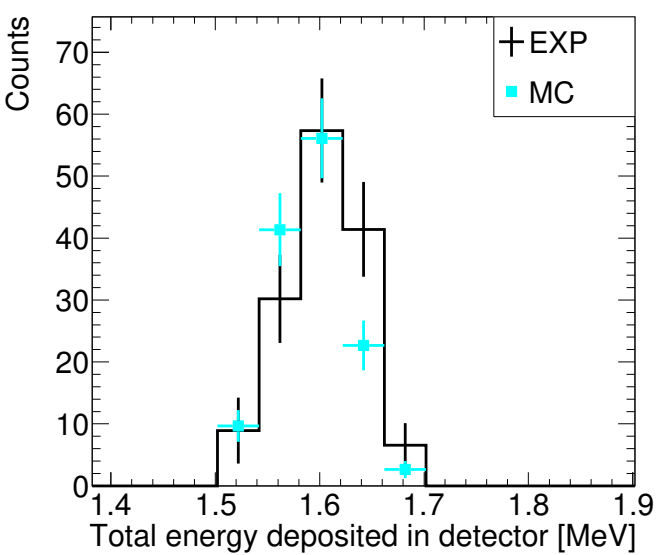

(e)

Figure 6.19: Simulated and experimental extended-source Compton imaging measurements at dwell point 1 after energy cuts. a) Compton cone polar angle. b) Compton scattering angle. c) Scatter-plane energies. d) Absorber-plane energies. e) Totalenergy depositions. 


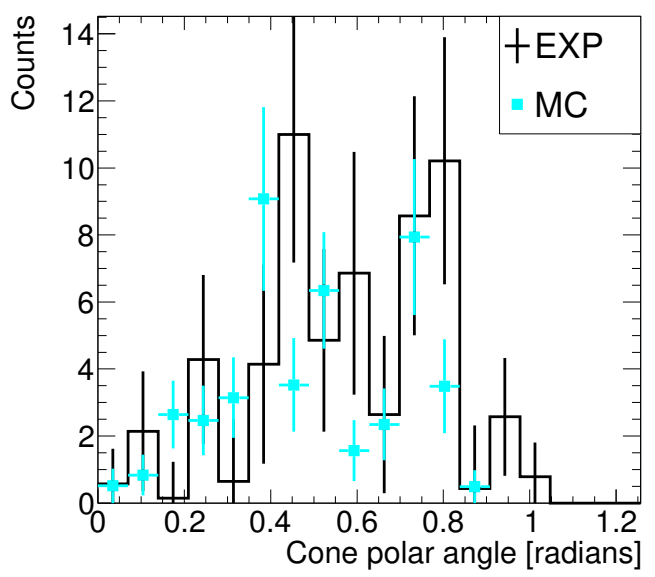

(a)

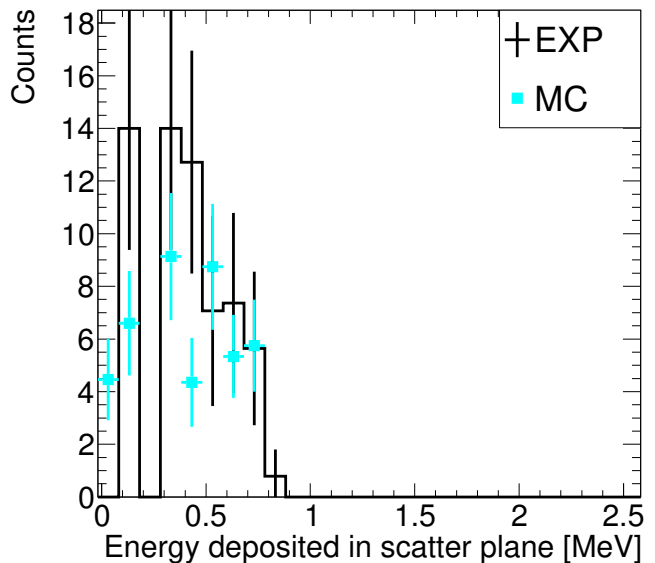

(c)

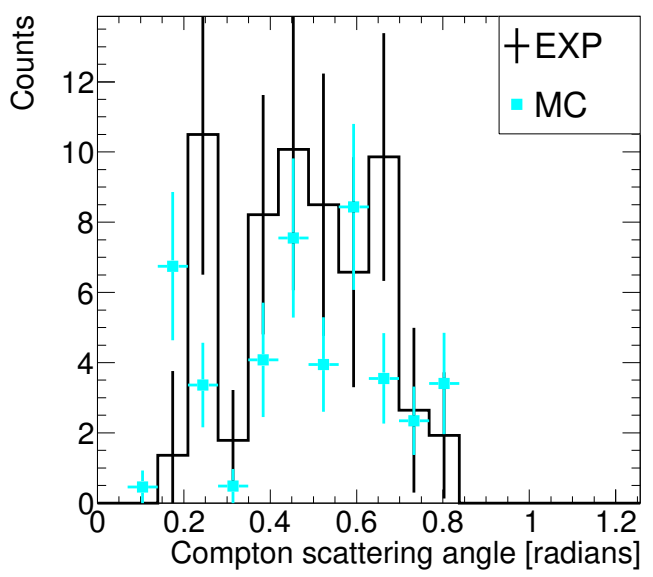

(b)

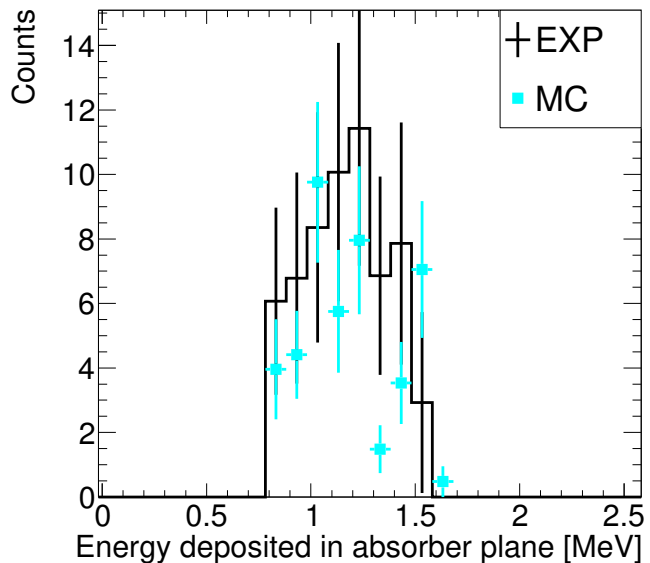

(d)

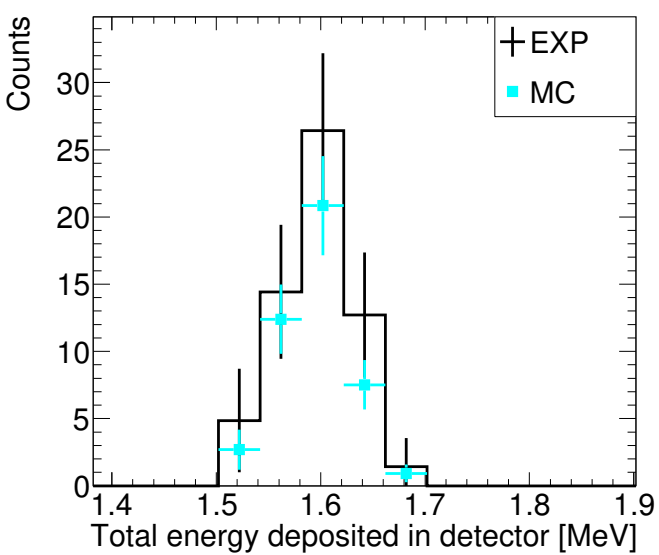

(e)

Figure 6.20: Simulated and experimental extended-source Compton imaging measurements at dwell point 2 after energy cuts. a) Compton cone polar angle. b) Compton scattering angle. c) Scatter-plane energies. d) Absorber-plane energies. e) Totalenergy depositions. 


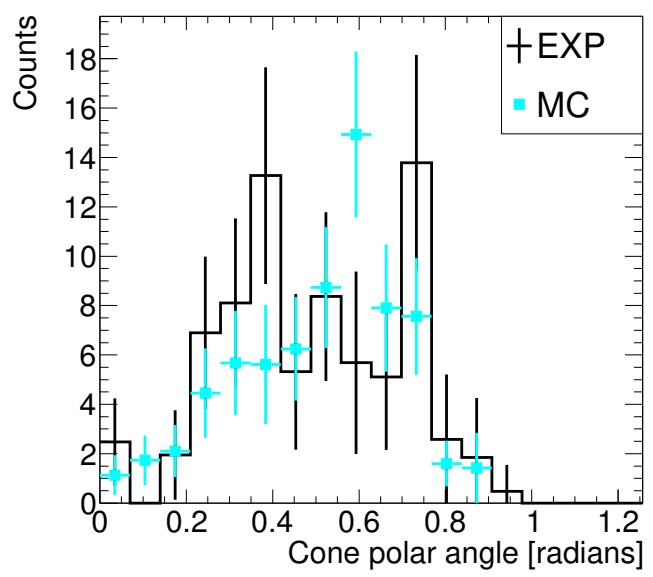

(a)

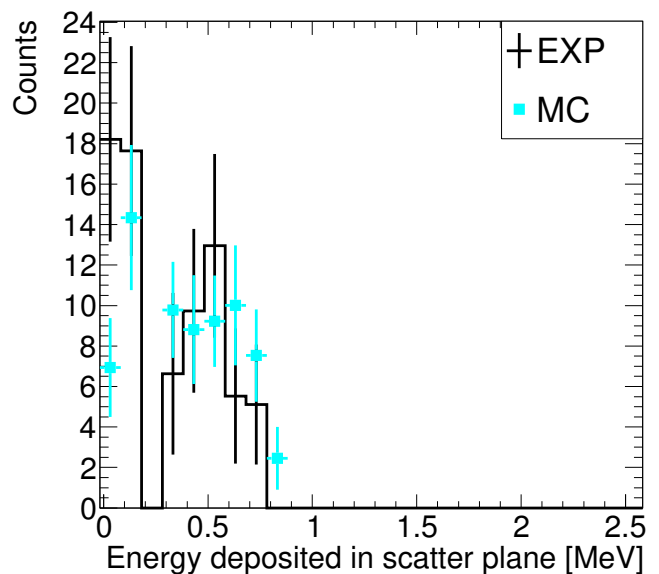

(c)

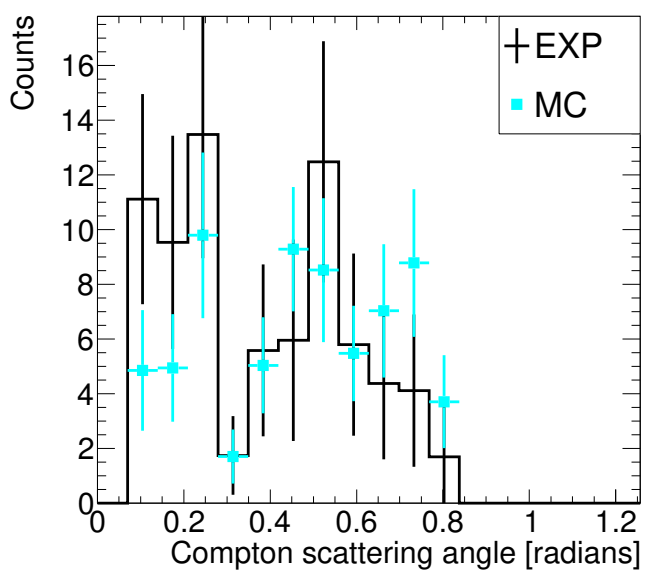

(b)

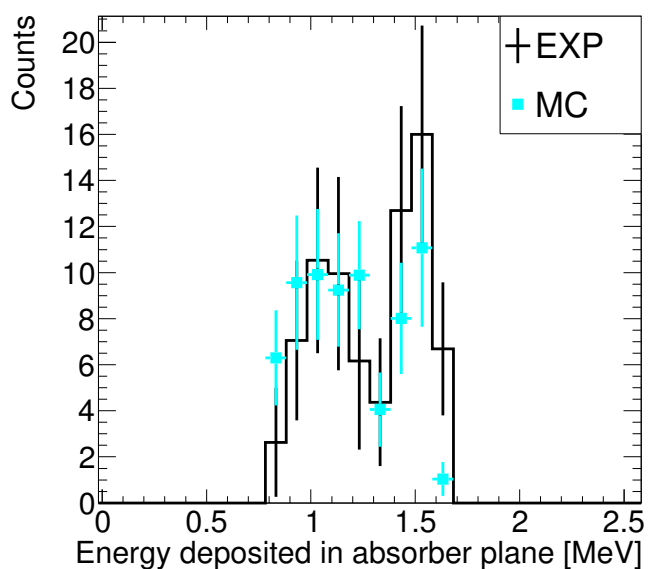

(d)

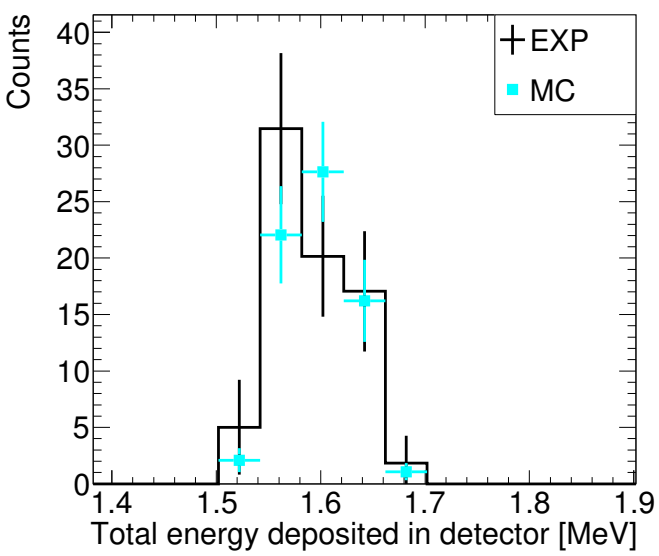

(e)

Figure 6.21: Simulated and experimental extended-source Compton imaging measurements at dwell point 3 after energy cuts. a) Compton cone polar angle. b) Compton scattering angle. c) Scatter-plane energies. d) Absorber-plane energies. e) Totalenergy depositions. 


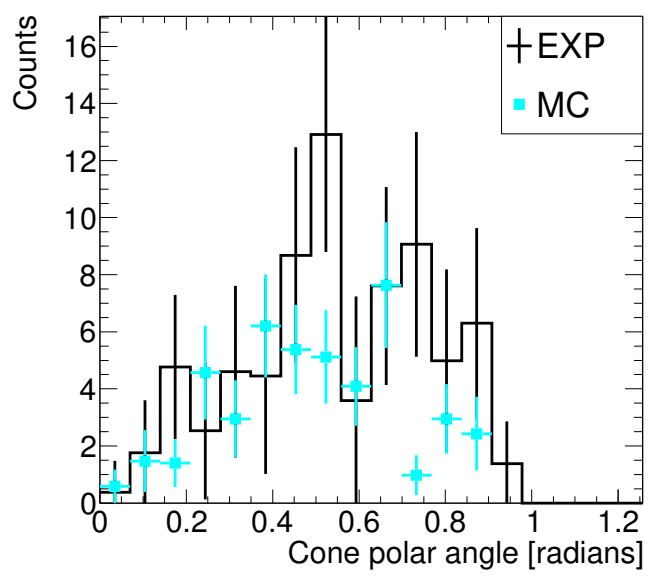

(a)

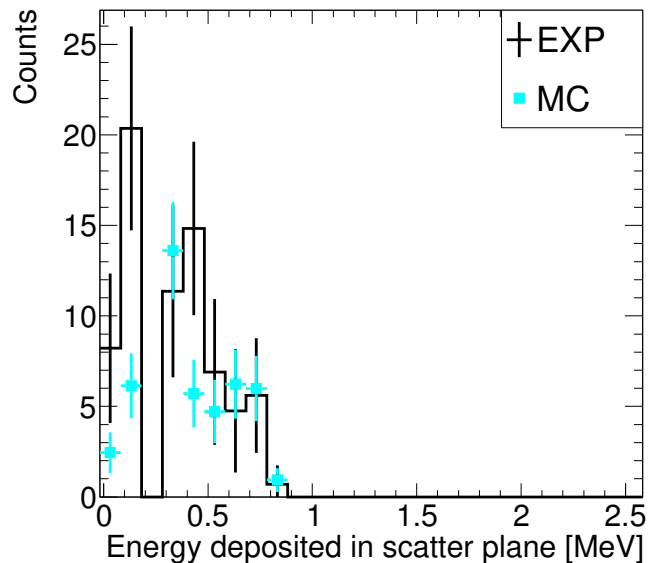

(c)

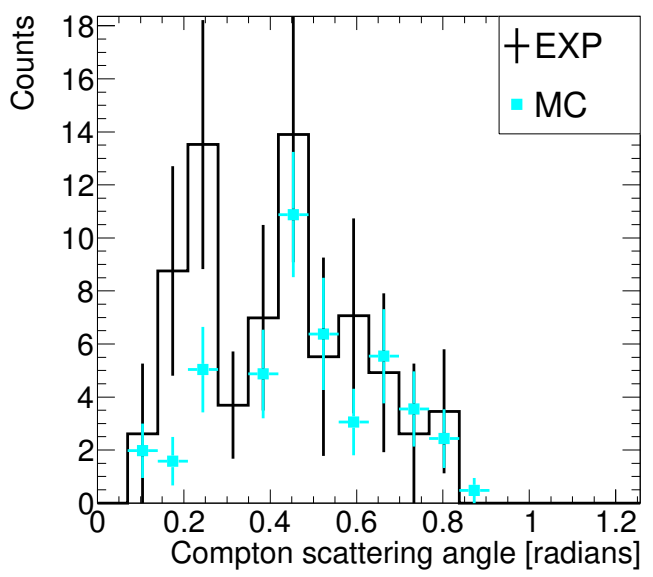

(b)

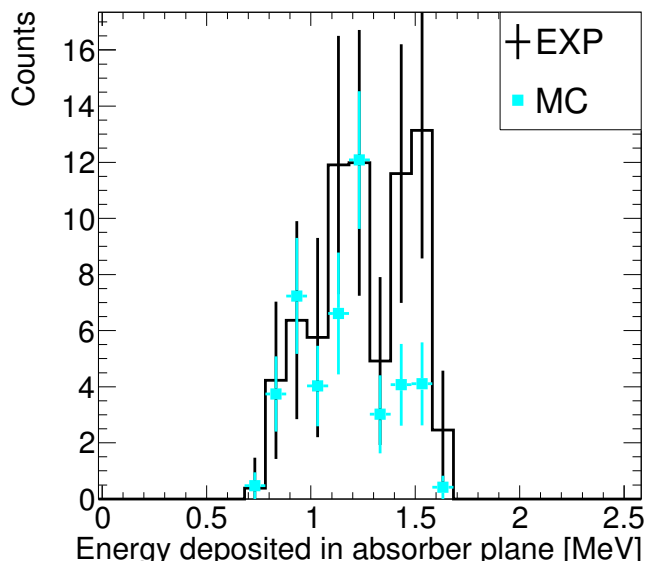

(d)

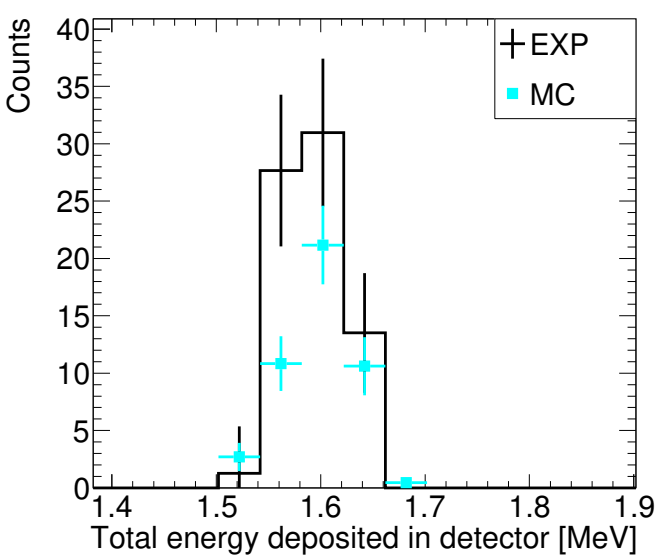

(e)

Figure 6.22: Simulated and experimental extended-source Compton imaging measurements at dwell point 4 after energy cuts. a) Compton cone polar angle. b) Compton scattering angle. c) Scatter-plane energies. d) Absorber-plane energies. e) Totalenergy depositions. 


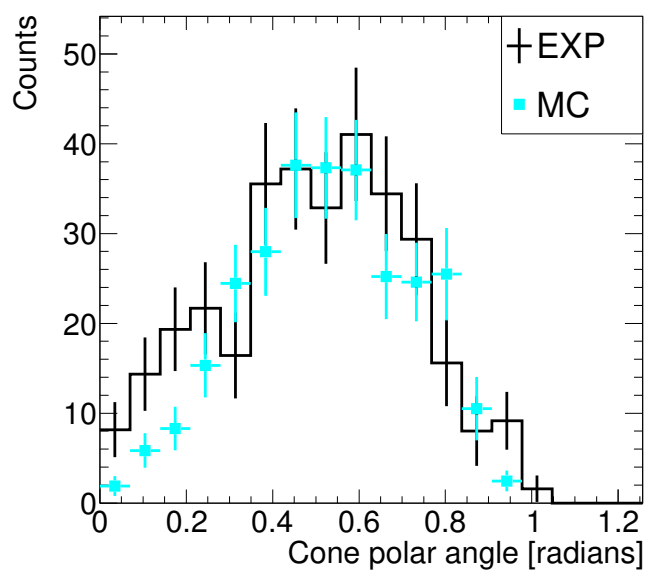

(a)

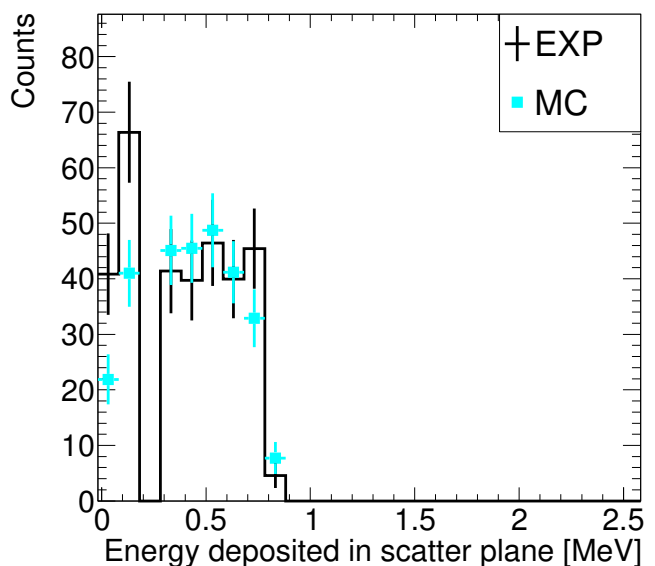

(c)

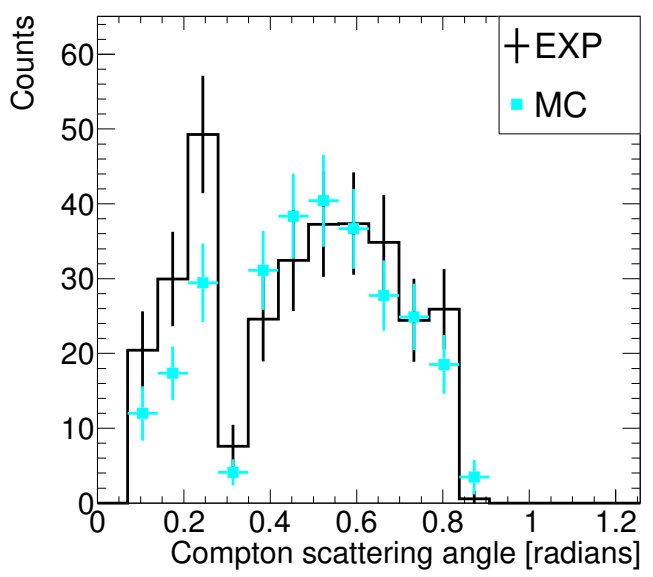

(b)

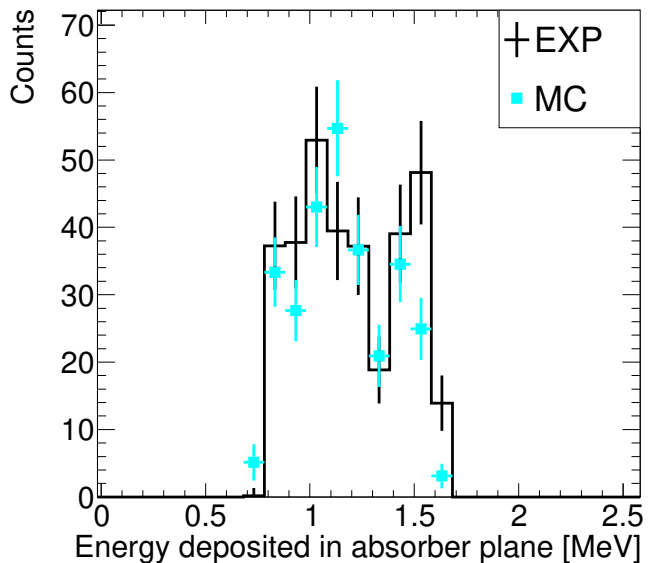

(d)

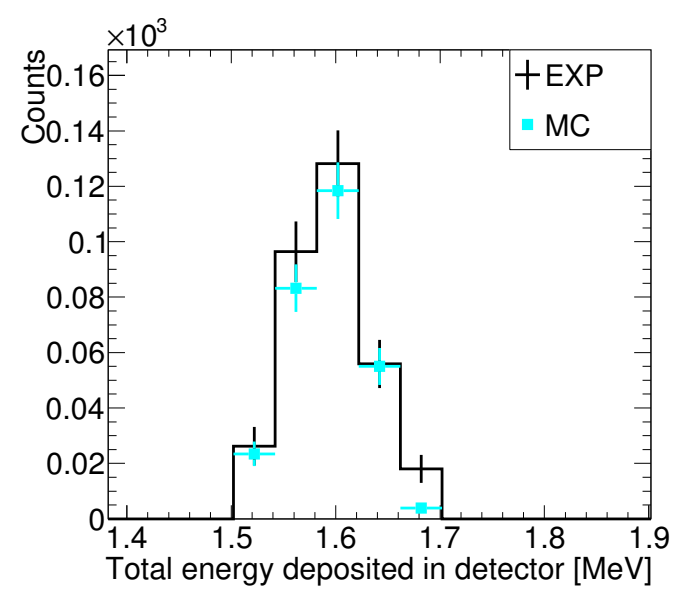

(e)

Figure 6.23: Simulated and experimental extended-source Compton imaging measurements at dwell point 5 after energy cuts. a) Compton cone polar angle. b) Compton scattering angle. c) Scatter-plane energies. d) Absorber-plane energies. e) Totalenergy depositions. 


\section{Point-model response}

The response of the detector's two-hit event rate to a point source as a function of distance and angle is required for the simple point-model estimate that accompanies the basic tomographic source distribution result (prior to unfolding). To parametrize the response of the $3 \times 3$-module SCoTSS detector to a point-source of La-140, point sources at increasing distances along the central axis of the detector ranging from $10 \mathrm{~m}$ to $600 \mathrm{~m}$ were simulated one-by-one using MC. The angular dependence of the response was parametrized by individually simulating point sources at increasing angles relative to the central axis of the detector of up to $80^{\circ}$, at a fixed radial distance of $50 \mathrm{~m}$. All simulations of point sources used one billion collimated histories, with the point source placed in the central plane of the detector in each simulation. A combined computation time of approximately 30 hours over 10 Intel Xeon E5620 processors was required per simulation.

The simulated data were then passed through the selection criteria presented in table 6.3 in order to determine the number of valid two-hit events. The results obtained for point sources at increasing distances were then normalized to the number of simulated decays to obtain a distance-dependent efficiency, and the results obtained for increasing angle relative to the detector were normalized to the value at the central axis to obtain a correction for the angular efficiency. Figure 6.24 shows the parametrizations obtained for the response to a point-source of La-140 that will be used in the point-model fit method for estimating the source activity rapidly. 


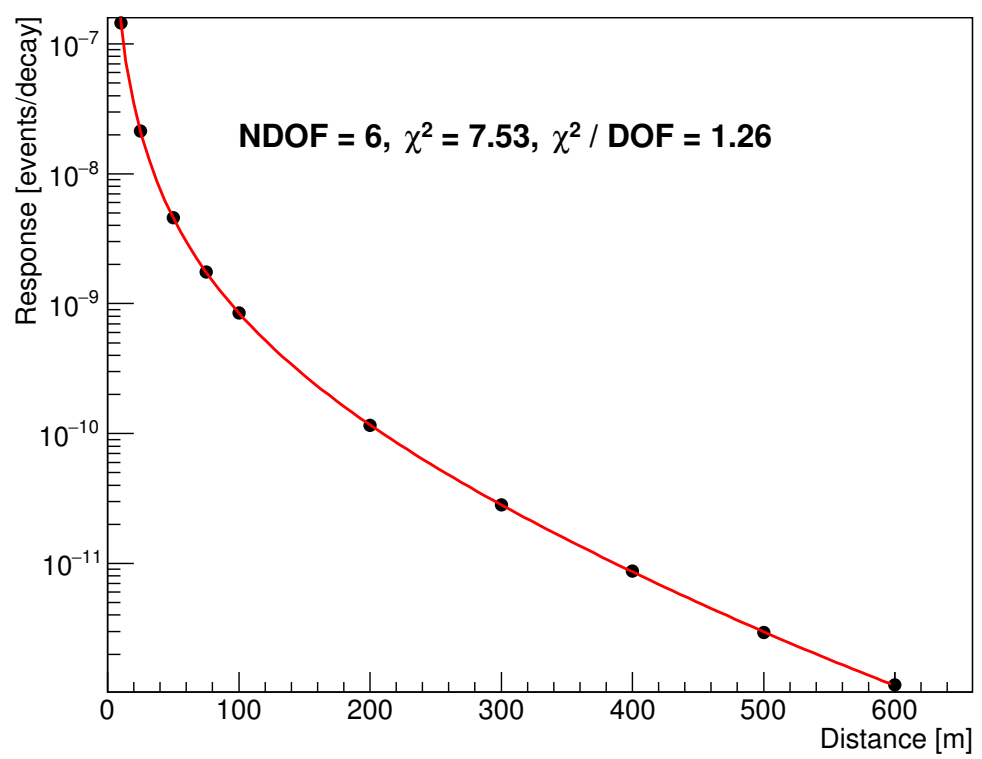

(a)

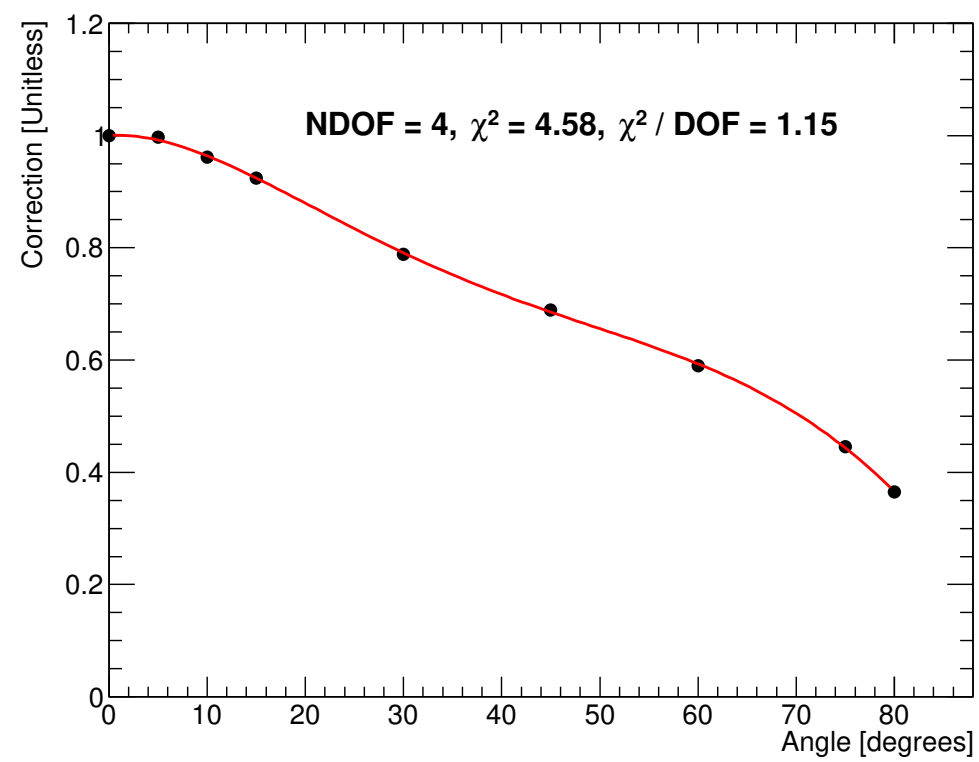

(b)

Figure 6.24: Point-response parametrization of an La-140 point source used in the Suffield experiment. a) Detector response with increasing distance along central axis, $f_{\text {dist }}(r)$. b) Correction for angular response, $f_{\text {ang }}\left(\theta_{\text {axis }}\right)$. In both subfigures, the uncertainty on the data points are too small to be visualized. 


\section{Verification of tomographic reconstruction using MC simulation}

A synthetic L-polygon source matching the dimensions of the planned source and with uniform surface activity concentration of La-140 was simulated in MC with an activity of $35 \mathrm{GBq}$ and with similar statistics to the experimental data. Figure 6.25a shows the tomographic reconstruction of the synthetic source, with symbols defined in the same way as in figure 6.1. A quality cut has been made to zero the pixels outside of the combined field of view of all six dwell points, representing an assumption that the source is enclosed by the dwell points. The tomographic reconstruction of the data places an extended band of high intensity in the vicinity of the large arm of the L-polygon, with evidence of an elevated region of intensity also along the small arm. These bands of high intensity cover a much larger spatial extent than is seen for the synthetic point source presented in section 5.1.3. Thus, the simple tomographic reconstruction method is able to provide evidence that the source is spatially extended. Furthermore, the two bands of high reconstructed intensity suggest that the source may extend in multiple directions.

The tomographic reconstruction displays the characteristic spoking artefacts found in tomographic back-projection, similar to those seen for the tomographic reconstruction of the synthetic point source shown in section 5.1.3 except for the fact that the spokes do not all intersect at a single location. Once again, the spatialunfolding method discussed later in this chapter will remove these artefacts. 


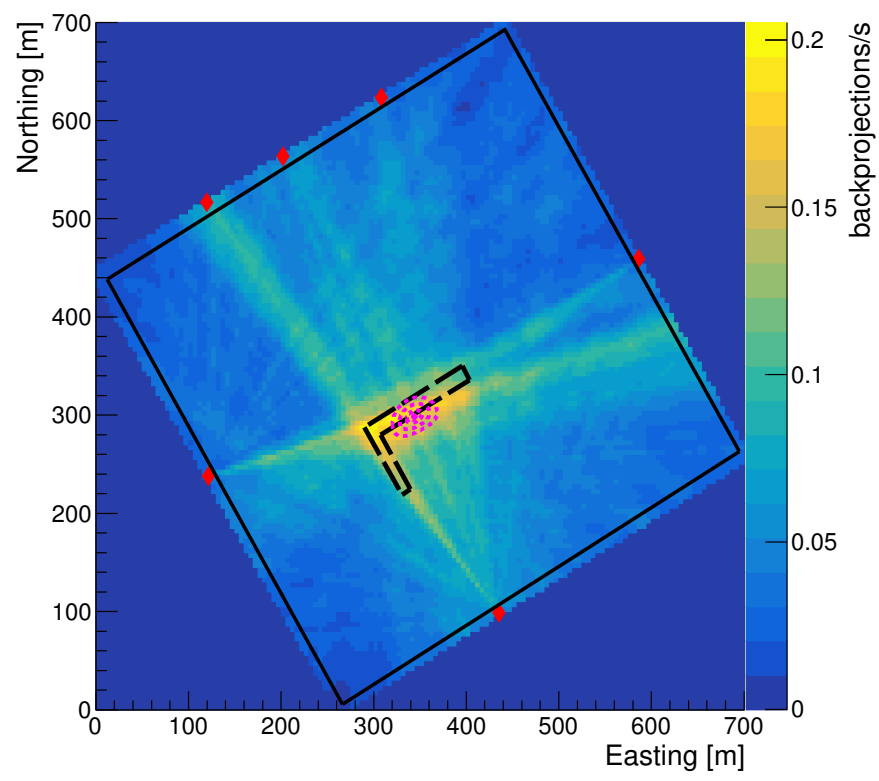

(a)

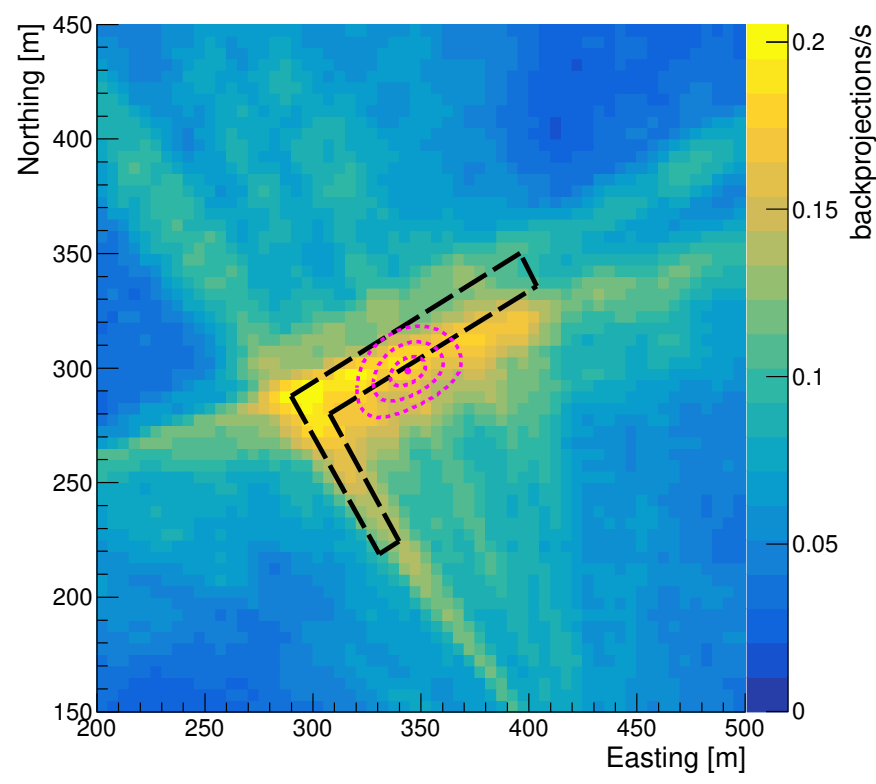

(b)

Figure 6.25: Tomographic reconstruction of synthetic La-140 "L-polygon" source with point-model approximation overlaid. a) View of full image space. b) Zoomed-in view at location of synthetic source. Symbols are defined as presented in figure 6.1, and the point-model fit result has additionally been overlaid; the magenta dot represents the placement of the fitted point model, and dashed magenta lines represent the $1 \sigma$, $2 \sigma$, and $3 \sigma$ spatial-uncertainty contours. The temporal rate arises from normalization by measurement time at each dwell point. 


\section{Verification of point-model response method using MC simulation}

Overlaid on the tomographic reconstruction in figure $6.25 \mathrm{a}$ is the point-model fit, and figure $6.25 \mathrm{~b}$ shows a zoomed-in view for clarity. A magenta dot denotes the placement of the point model, and dashed magenta lines represent the $1 \sigma, 2 \sigma$, and $3 \sigma$ spatial-uncertainty contours. The uncertainties were scaled by a factor of $\sqrt{3}$ in the point-model fit to bring the $\chi^{2}$ per degree of freedom to 1 , a step made necessary due to the fact that a point model is inconsistent with the L-polygon source. Scaling the errors to bring the $\chi^{2}$ per degree of freedom to 1 thus provides uncertainty estimates that are of the appropriate scale.

The fit placed the point source at $(344 \pm 8) \mathrm{m}$ along the easting axis and (299 \pm 6) $\mathrm{m}$ along the northing axis, and assigned an activity of $(37 \pm 2) \mathrm{GBq}$ to the source. The reconstructed activity therefore agrees with the expected value of $35 \mathrm{GBq}$ at a significance level of 0.01 , obtaining a Z-score of 1.00 and a two-sided P-value of 0.32. The placement of the point-model fit is also broadly in agreement with the placement of the L-polygon source. The centroid of the large arm of the L-polygon sat at $(347,312) \mathrm{m}$ and the centroid of the small arm sat at $(315,256) \mathrm{m}$. The location of the fit is near the middle of the long axis of the large arm of the L-polygon, and towards the side along which the small arm extends. This suggests that the fit may be sensitive to the radiation contained in the small arm of the L-polygon. However, because the large arm of the L-polygon contains $80 \%$ of the simulated decays, the signal from the large arm dominates. The spatial-uncertainty contours on the fit primarily extend along the direction of the large arm of the L-polygon, demonstrating the sensitivity of the fit to the underlying distributed nature of the source.

\section{Response templates}

For the application of the spatial-unfolding method, the response of the $3 \times 3$-module SCoTSS detector to spatially-localized sources of La-140 is required. The template response of the 3x3-module SCoTSS detector to spatially-localized sources of La-140 was obtained by simulating a $500 \mathrm{~m}$ by $500 \mathrm{~m}$ uniform sheet source of La-140, chosen to match the size of the restricted-access zone, in the appropriate location relative to each of the six dwell points in order to reproduce the measurement geometry of the

Suffield experiment. The $500 \mathrm{~m}$ by $500 \mathrm{~m}$ sheet source was placed in the same plane

\subsection{METHODOLOGY}


as the detector in each dwell point simulation, and 30 billion collimated histories were simulated over 250 Intel Xeon E5620 processors, requiring a combined total computation time of approximately 1350 hours per simulated dwell point. The use of a single $500 \mathrm{~m}$ by $500 \mathrm{~m}$ sheet source allows for the simulated response of the detector to be obtained for all regions of the restricted-access zone simultaneously, and the data can be sorted in post-processing using the known emission locations of the primary photons in order to obtain the response to specific regions within the zone.

Using the known emission location of each primary photon in the simulation, the simulated data were divided into $50 \mathrm{~m}$ by $50 \mathrm{~m}$ tiles in order to produce 100 spatially-localized sources; these correspond to the $f_{i}$ described in section 4.8. This sorting process took approximately two hours to complete on a single $3.1 \mathrm{GHz}$ Intel Core i5 processor. For each $f_{i}$, the simulated photons that reach the detector at each dwell point, deposited energy in one crystal in the scatter plane and in one crystal in the absorber plane, and that passed the selection criteria given in table 6.3 were then used to reconstruct a tomographic response template $g_{i}$ by passing the data through the reconstruction chain described in section 4.5 followed by normalization of the response template by the simulated number of decays. This last step took approximately two hours to complete on a single $3.1 \mathrm{GHz}$ Intel Core i5 processor.

\subsection{Results and discussion}

\subsubsection{Uncorrected tomographic reconstruction}

A simplifying assumption was made during reconstruction of the data acquired in the Suffield experiment that the La-140 source was distributed along the horizon in the field of view of the $3 \times 3$-module SCoTSS detector (i.e. at a vertical angle of $0^{\circ}$ in the field of view). This reduced the problem to the reconstruction of a two-dimensional tomographic image from individual one-dimensional images at each dwell point. The low height of the detector when atop the BV206 vehicle, along with the relatively flat topography of the restricted-access zone (Google Earth data indicates that the zone varies by no more than approximately $5 \mathrm{~m}$ across its extent), justify this assumption. Though it may be noted that a source on the surface of a flat earth would still subtend 
a non-zero vertical angle for points close to the detector given its $2.5 \mathrm{~m}$ height, the assumption that the source was at the horizon in the field of view of the detector was a good approximation for this particular experiment as the source was always at a distance of at least $175 \mathrm{~m}$ from the detector.

An additional quality cut was enforced which zeroed pixels outside of the combined field of view of all six dwell points. This quality cut represents an assumption that the La-140 was contained exclusively inside of the restricted-access zone.

Figure 6.26 shows the ARM distribution obtained for a synthetic La-140 point source at $250 \mathrm{~m}$ distance, generated using the MC representation described in section 6.1.4 and selecting for the $1.596 \mathrm{MeV}$ gamma-ray emission. The ARM distribution had a standard deviation of $2.89^{\circ}$. A back-projected ray width of $3^{\circ}$ was therefore chosen for tomographic reconstruction.

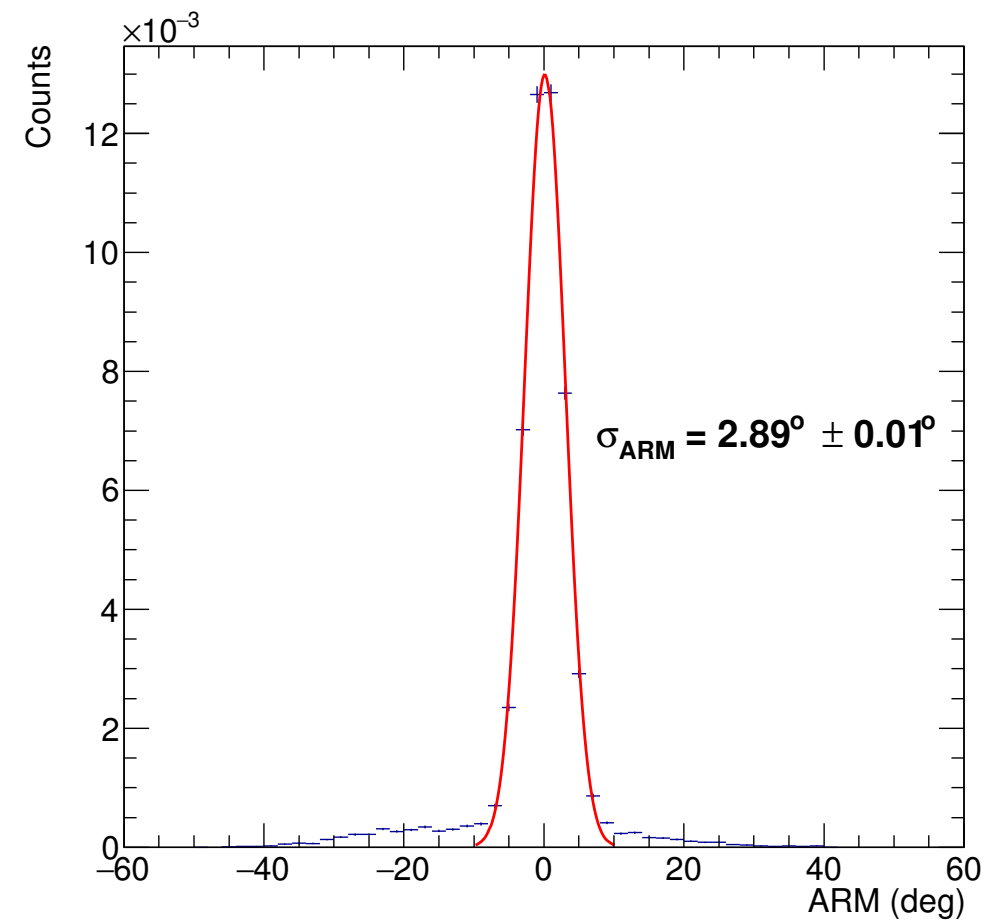

Figure 6.26: ARM distribution for a synthetic La-140 point source.

Figure 6.27 presents a background-subtracted tomographic reconstruction of the distribution of La-140, with symbols defined in the same way as was presented for 
figure 6.1. The tomographic reconstruction of the data demonstrates a band of high

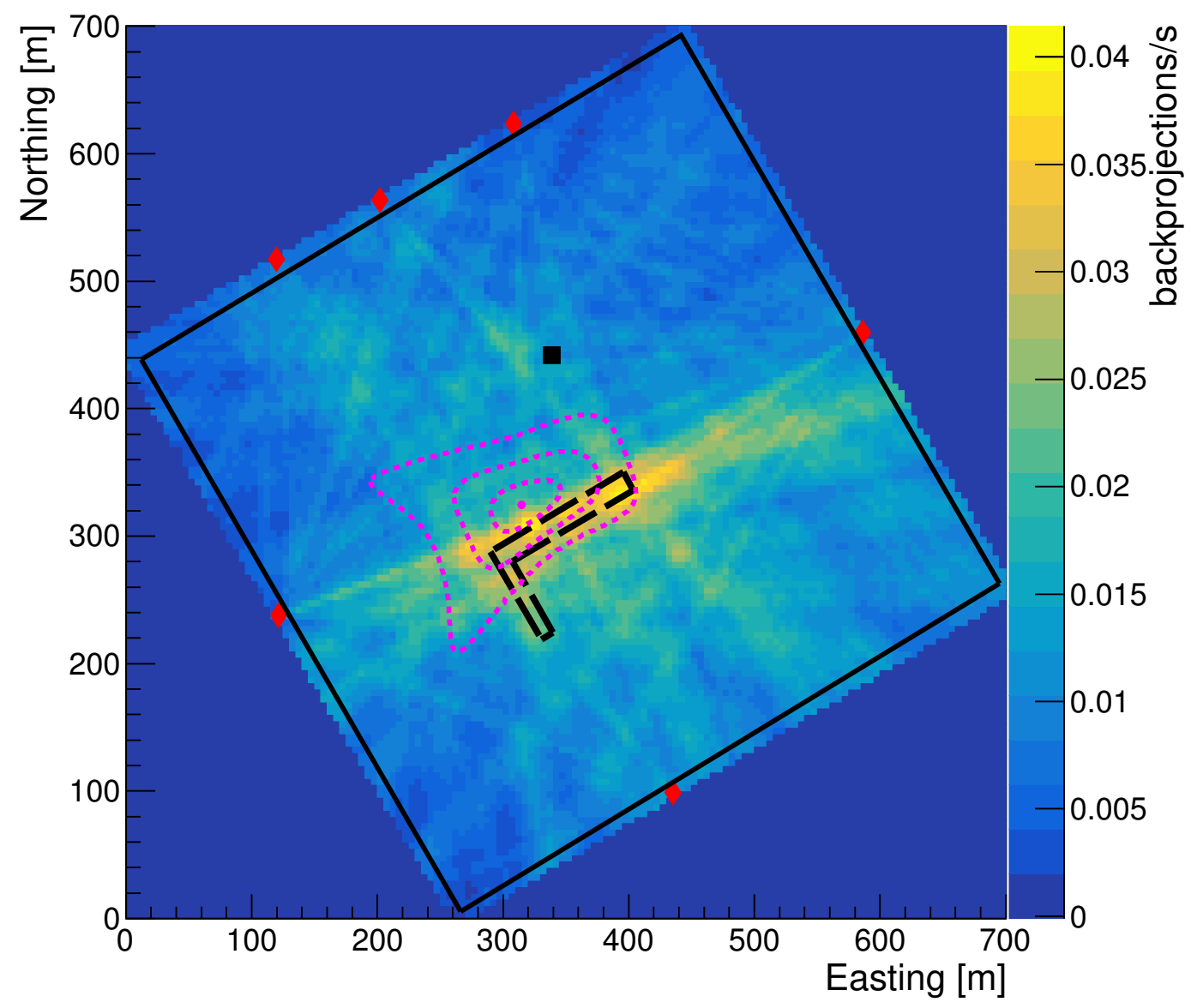

Figure 6.27: Tomographic reconstruction of experimental La-140 source with pointmodel approximation overlaid. Symbols are defined as presented in figure 6.1, and the point-model fit result has additionally been overlaid; the magenta dot represents the placement of the fitted point model, and dashed magenta lines represent the $1 \sigma, 2 \sigma$, and $3 \sigma$ spatial-uncertainty contours. The temporal rate arises from normalization by measurement time at each dwell point.

reconstructed intensity extending from the south-west to the north-east. The highintensity region coincides with the large arm of the L-polygon shape within which the La-140 was deposited. There is little evidence of reconstructed intensity inside the small arm of the L-polygon. This is likely due to the imbalance in the La-140 concentrations between the arms of the L-polygon that resulted from the sprayer malfunction, as extra La-140 was placed in the large arm. The imbalance between 
the arms of the L-polygon was independently verified by a UAV-mounted gamma spectrometry system. ${ }^{2}$ Additionally, the limited number of dwell points utilized may not have allowed for reconstruction of the weaker signal in the small arm of the L-polygon.

The tomographic reconstruction of the data in figure 6.27 contains spoking artefacts which can be observed extending from each dwell point towards the region of maximal intensity. These spoking artefacts are expected, and have been demonstrated in the validation of the tomographic reconstruction method as shown in figure 6.25a. However, unlike in the tomographic reconstruction presented in figure 5.35 for an experimentally-measured point source, the spoking artefacts do not intersect within a relatively localized region. This provides further evidence that the La-140 is spatially distributed.

The reconstruction took approximately 90 seconds to complete on a laptop computer with a $3.1 \mathrm{GHz}$ Intel Core 15 processor, demonstrating the ability of the tomographic reconstruction method to quickly locate a spatially-extended source in operational conditions in near real time.

\subsubsection{Point-model estimate}

The point-model fit has been overlaid on the tomographic reconstruction in figure 6.27. A magenta dot represents the placement of the fitted point model, and dashed magenta lines represent the $1 \sigma, 2 \sigma$, and $3 \sigma$ spatial-uncertainty contours. The fit placed the point source at $(315 \pm 26) \mathrm{m}$ along the easting axis and $(324 \pm 19) \mathrm{m}$ along the northing axis, and assigned an activity of $(7 \pm 2) \mathrm{GBq}$ to the source. In order to obtain the estimated uncertainties on the fit result, the uncertainties on the counts were scaled upwards by a factor of $\sqrt{52}$ in order to bring the $\chi^{2}$ per degree of freedom to 1 . This step was undertaken to ensure proper scaling of the uncertainties, and was required because the hypothesized point model differs from the known spatially-extended La-140 distribution.

The location of the point-model fit is just adjacent to the expected location of the large arm of the L-polygon and is between the L-polygon and the final location of the sprayer UGV. The spatial-uncertainty contours on the point-model fit extend primarily along the direction of the large arm of the L-polygon. This suggests that the 
fit is sensitive to the fact that the underlying La-140 distribution is spatially extended, and requires larger variance along the axis of the source with more contamination. The $2 \sigma$ contour largely overlaps the large arm of the L-polygon, and the large arm of the L-polygon is entirely enclosed within the $3 \sigma$ contour; this suggests that the pointmodel fit is not in disagreement with the expected placement of the La-140. Residual La-140 contamination on the sprayer UGV may have pulled the point-model fit away from the L-polygon, and unknown amounts of intermittent snow and soil attenuators not included in the MC simulations of the detector's response may have additionally influenced the fit result.

Comparison of the activity estimate of $(7 \pm 2) \mathrm{GBq}$ to the expected $35 \mathrm{GBq}$ yields a Z-score of -14.0 and a two-sided P-value of 0.00 . The activity estimated by the point-model fit is therefore in disagreement with the expected value of $35 \mathrm{GBq}$ at a significance level of 0.01. A more detailed discussion of possible reasons for this is provided in section 6.2.3 as the total activity estimated by the point-model method is in agreement with that predicted by the spatial-unfolding method discussed in that section. In short, it is believed that environmental terrain not included in the simulated response of the detector caused signal loss that was not accounted for by the response parametrization.

\subsubsection{Spatially-unfolded distribution}

\section{Tomographic fit}

The tomographic reconstruction methodology outlined in section 4.5 and the spatialunfolding methodology described in section 4.8 were used in the reconstruction of the experimental data. In the application of the minimization, it was assumed that there was no activity in the pixels immediately adjacent to the border of the restrictedaccess zone and the value of $w_{i}$ associated with them was fixed to zero within the Minuit2 minimizer.

The spatial-unfolding results are presented in a coordinate system relative to the south-western border of the restricted-access zone shown in figure 6.1. This is done for convenience when generating the response functions. Figure 6.28 shows a schematic of the relative coordinate system, with symbols defined the same was as in 
figure 6.1 .

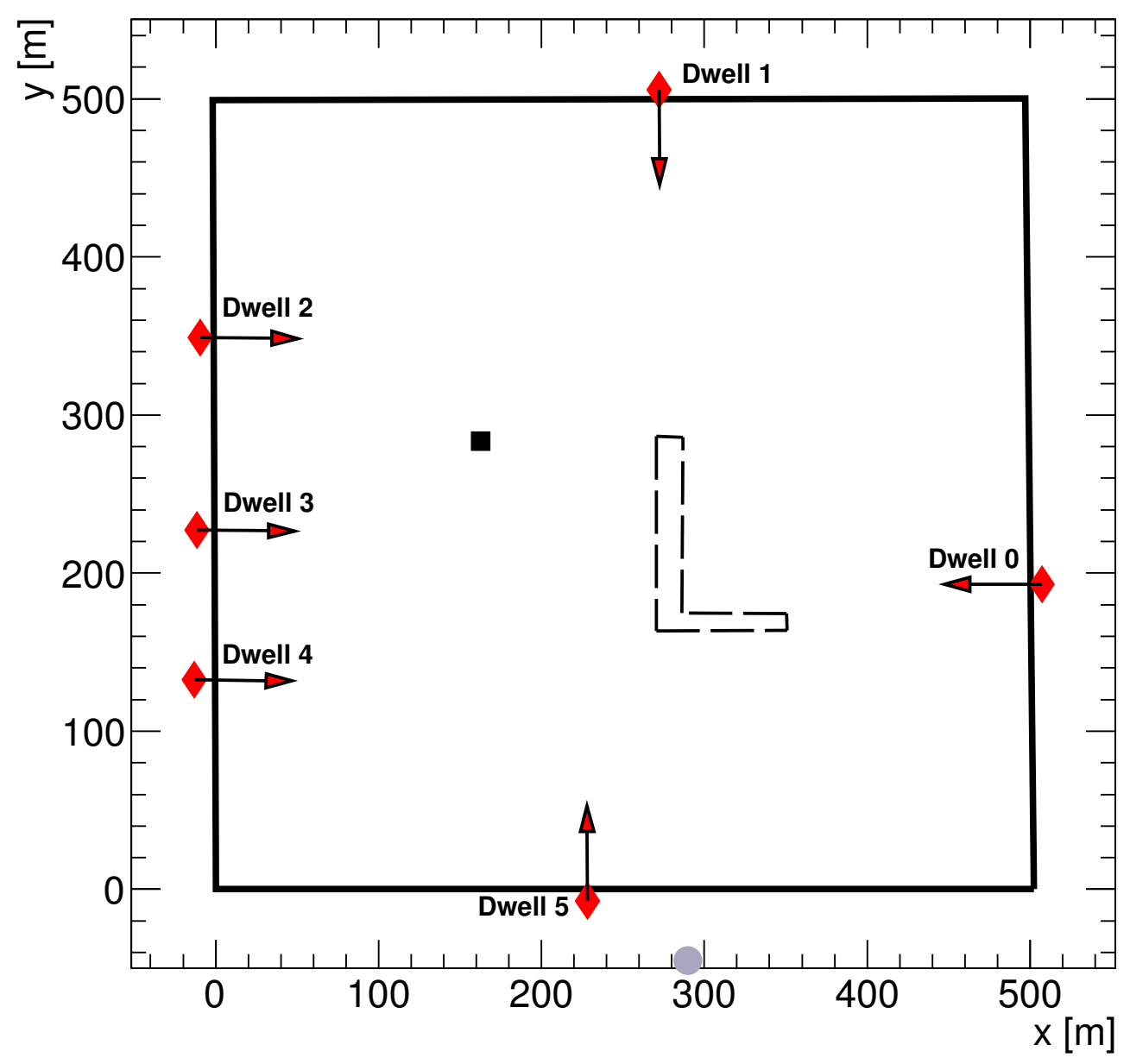

Figure 6.28: Schematic representation of the Suffield experimental site in coordinates relative to south-western border, used for presentation of the spatially-unfolded results. The symbols are defined the same way as in figure 6.1.

Given the limited statistical power of this measurement and the complexity in unfolding a large number of parameters, a $50 \mathrm{~m}$ by $50 \mathrm{~m}$ binning was chosen for the representation of the tomographic reconstruction for unfolding. Figure 6.29a shows the tomographic reconstruction of the La-140 in the $50 \mathrm{~m}$ by $50 \mathrm{~m}$ binning chosen for the unfolding. The expected location of the La-140 is indicated by dashed black lines, a closed black square marks the UGV sprayer, and the dwell points are not visible in the coordinate system that has been presented (they are just outside the limit 
of the coordinates which are shown and which correspond entirely to the interior of the restricted-access zone). A region of high intensity has been reconstructed along the large arm of the L-polygon, and the tomographic reconstruction of the data demonstrates spoking artefacts that contribute to a smearing of the distribution.

Figure $6.29 \mathrm{~b}$ shows the result of fitting the simulated response templates to figure 6.29a. The fit shares the general patterns found in the tomographic result; maximal intensity has been placed along the large arm and there is evidence of spoking that extends towards the edges of the image space. Figures $6.29 \mathrm{c}$ and $6.29 \mathrm{~d}$ show vertical profiles taken at $x=275 \mathrm{~m}$ and horizontal profiles taken at $y=275 \mathrm{~m}$, respectively. The fit tomogram generally shows good agreement with the data tomogram, as evidenced by the one-dimensional profiles.

The magnitude of the fit tomogram within the planned L-polygon is lower than that of the data tomogram. However, as shown in section 5.2.3 this is likely due to the fact that the size of the source was not commensurate with that of the response templates. To show that this effect persists in the measurement geometry of the Suffield experiment, synthetic data were generated from the unfolded distribution of La-140 (which is presented further below in this section) in MC simulation with comparable statistics to the experimental data. The simulated source was therefore composed of La-140 "tile sources" that were the same size and shape as the response templates. Figure 6.30 shows the resulting tomographic reconstruction and fit result, with subfigures defined the same way as in figure 6.29. The fit shown in figure $6.30 \mathrm{~b}$ demonstrates good reproduction of the synthetic data tomogram presented in figure 6.30a, which is further evidenced by the one-dimensional profiles shown in figures $6.30 \mathrm{c}$ and $6.30 \mathrm{~d}$. This supports the previous findings that it is the difference in shape and size between the response templates and the underlying source that contributes to a tendency to underestimate the tomographic reconstruction of the data. It was shown in section 5.2.3 using a synthetic point source that this effect does not compromise the ability of the method to reproduce the total activity of the underlying source. 


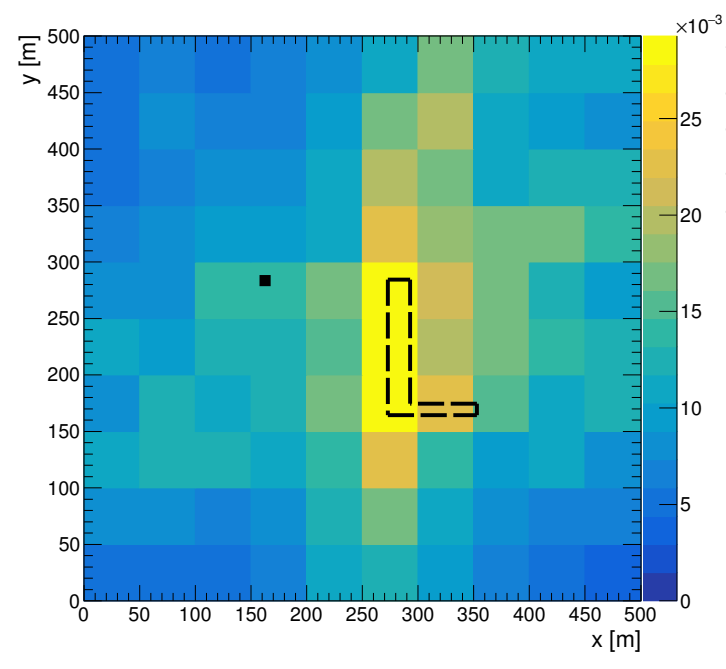

(a)

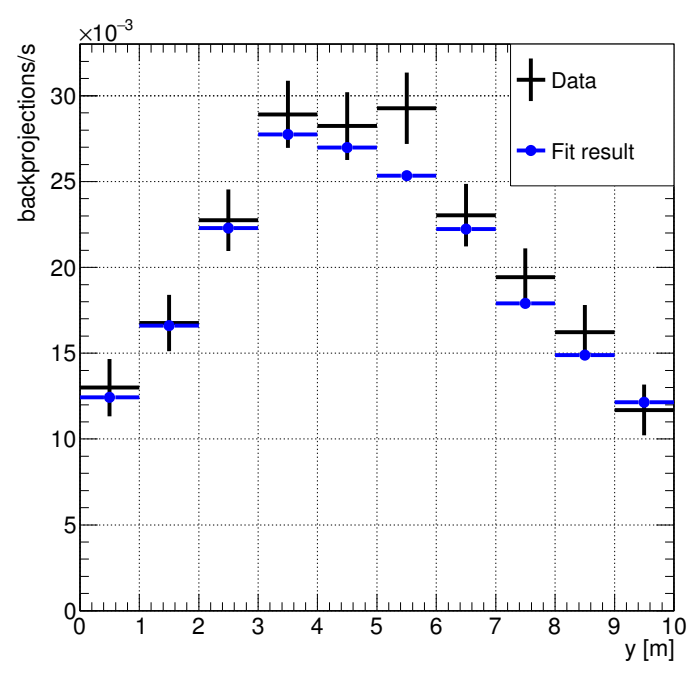

(c)

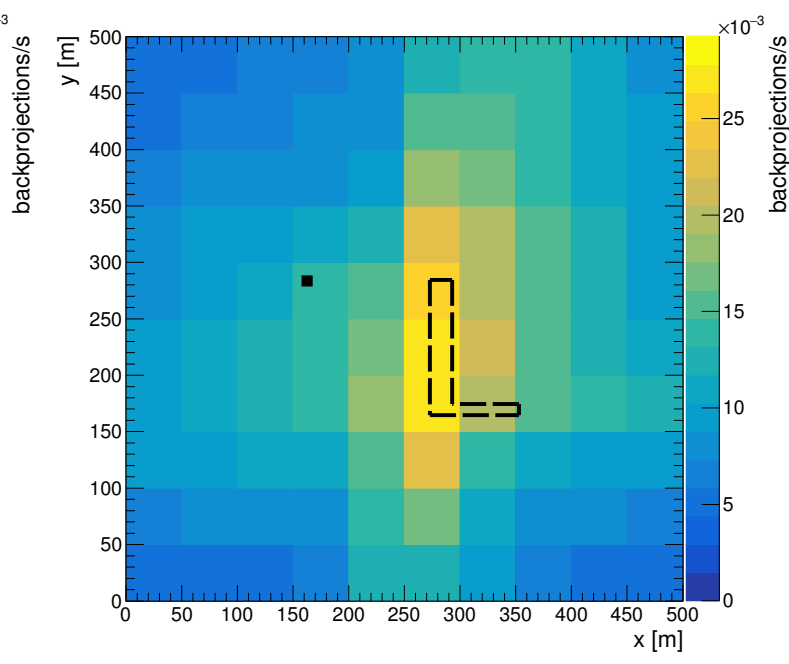

(b)

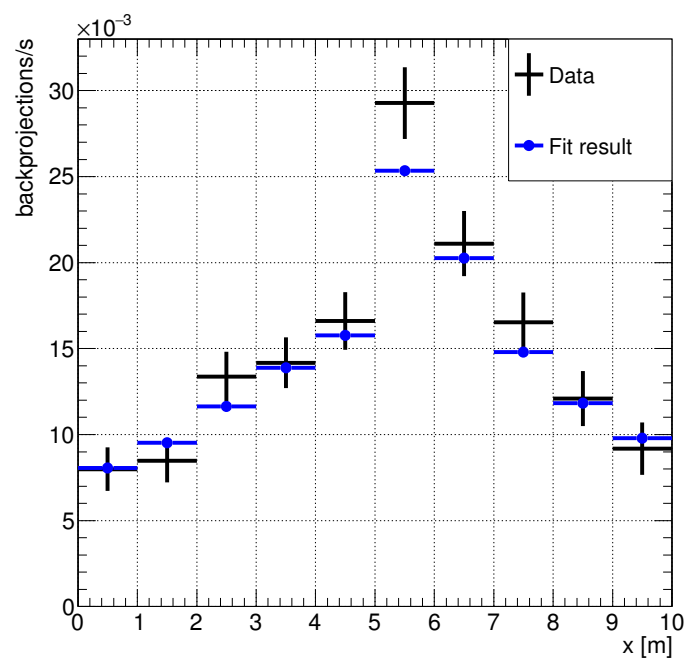

(d)

Figure 6.29: Tomographic reconstruction of experimentally-measured extended source with fit to data tomogram produced by spatial unfolding. a) Tomographic reconstruction of La-140 distribution. b) Fit to data tomogram produced by the minimization used in spatial unfolding. c) Vertical profiles of figures a and $b$ taken at $x=275 \mathrm{~m}$. d) Horizontal profile of figures $a$ and $b$ taken at $y=275 \mathrm{~m}$. All figures are presented in coordinates relative to the south-western border of the restricted access zone and on the same colour scale. Dashed black lines denote the expected distribution of La-140 and the closed black square marks the placement of the sprayer $U G V$ in figures a and $b$. 


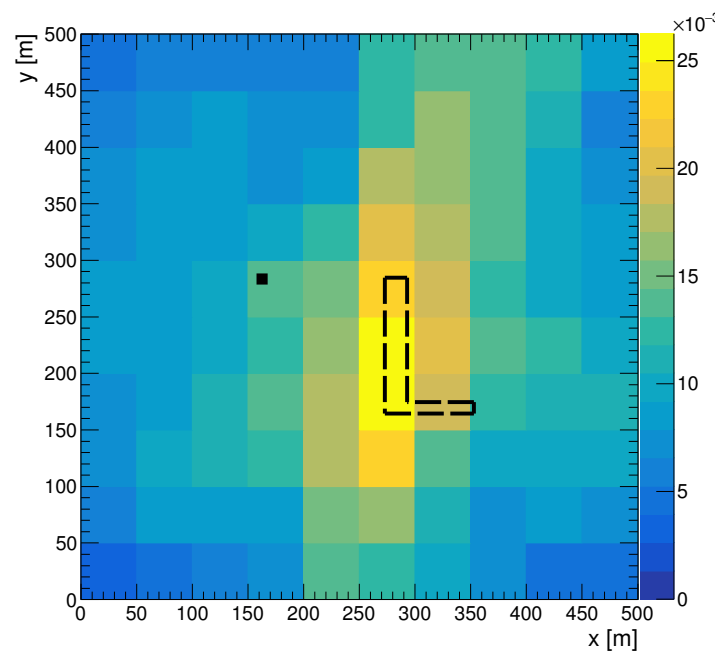

(a)

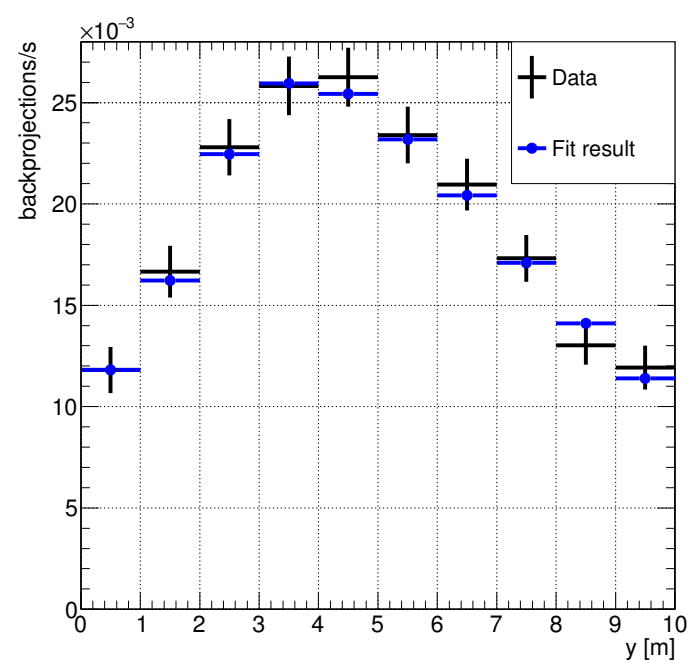

(c)

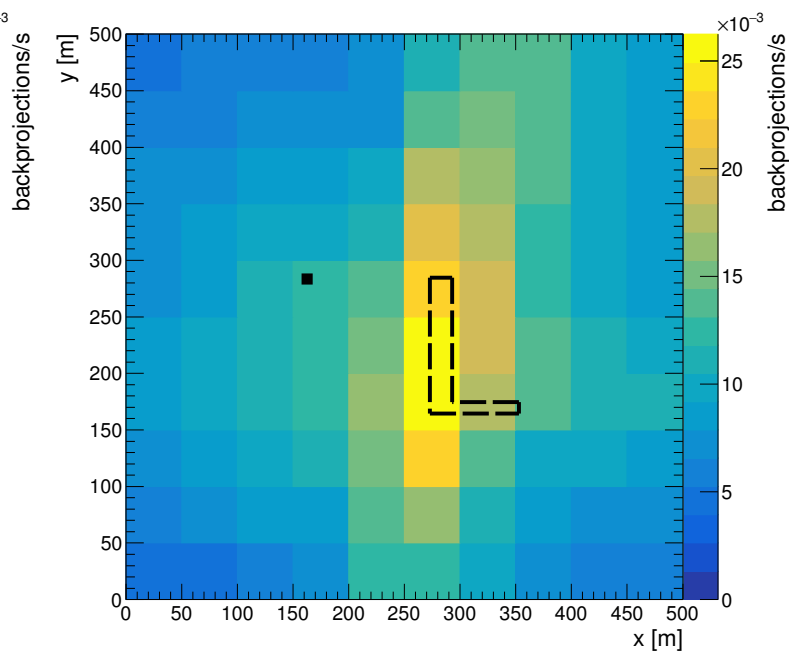

(b)

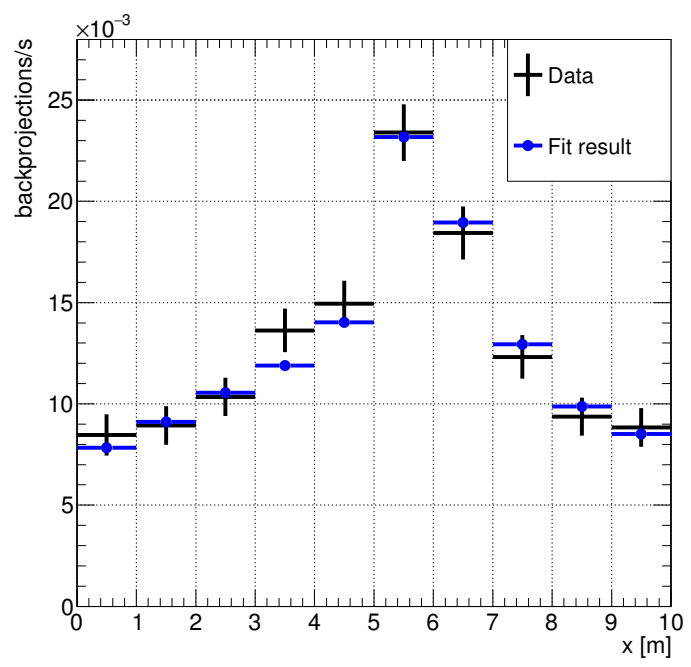

(d)

Figure 6.30: Tomographic reconstruction and fit result for synthetic data made from simulation of unfolded La-140 distribution. a) Tomographic reconstruction of La-140 distribution. b) Fit to data tomogram produced by the minimization used in spatial unfolding. c) Vertical profiles of figures $a$ and $b$ taken at $x=275 \mathrm{~m}$. d) Horizontal profile of figures $a$ and $b$ taken at $y=275 \mathrm{~m}$. All figures are presented in coordinates relative to the south-western border of the restricted access zone and on the same colour scale. Dashed black lines denote the expected distribution of La-140 and the closed black square marks the placement of the sprayer $U G V$ in figures a and $b$. 


\section{Unfolded distribution and uncertainties}

Figure 6.31a shows the estimated distribution $\tilde{f}$ of La-140 that results from unfolding the data tomogram presented in figure 6.29a using the fit result given in figure 6.29b, in units of $\mathrm{Bq} / \mathrm{m}^{2}$, where the branching ratio of La-140 has been used to recover units of activity. The spatial unfolding algorithm has eliminated the smearing and spoking that was present in the original tomographic reconstruction of the data and has placed estimates of activity at the location of the sprayer UGV and in the vicinity of the planned L-polygon. In total, the surface area within which the unfolded distribution places estimates of La-140 is $17,500 \mathrm{~m}^{2}$, representing $7 \%$ of the area of the entire image space. This result therefore drastically reduces the area within which La-140 may be suspected to lie.

The statistical uncertainties were estimated as described in section 4.8. For the repeated MC simulation method, the unfolded estimate $\tilde{f}$ shown in figure 6.31a was built in EGSnrc and 25 independent simulation datasets were generated. For the bootstrapping method, 25 bootstrapped datasets were generated. Each of the datasets were then reconstructed, and the standard deviation of the content of each pixel used as an estimate of its statistical uncertainty. Figure 6.31c shows an estimate of the statistical uncertainties obtained by the repeated MC simulation method, while figure $6.31 \mathrm{~d}$ shows an estimate of the statistical uncertainties obtained by the bootstrapping method. Both estimates of the statistical uncertainty have been presented on the same colour scale as $\tilde{f}$ in figure 6.31a. The methods give similar estimates of the statistical uncertainty at the location of the planned L-polygon and at the location of the sprayer UGV. Within the $2 \sigma$ estimate of the statistical uncertainty each method shows that there is a significant amount of La-140 in the large arm of the L-polygon and at the sprayer UGV; this is true at the $1 \sigma$ level for the small arm of the L-polygon, which was difficult to resolve in the uncorrected tomographic reconstruction of the data. Both estimates of the statistical uncertainty suggest that there may be La-140 in the pixels just above and just below the large arm of the L-polygon, as the statistical uncertainty estimates alone are not sufficient to bring the unfolded La-140 amounts to $0 \mathrm{~Bq} / \mathrm{m}^{2}$ in these regions.

The total systematic uncertainty on $\tilde{f}$ was estimated by varying different parameters during the reconstruction, each of which is described in the list below. 


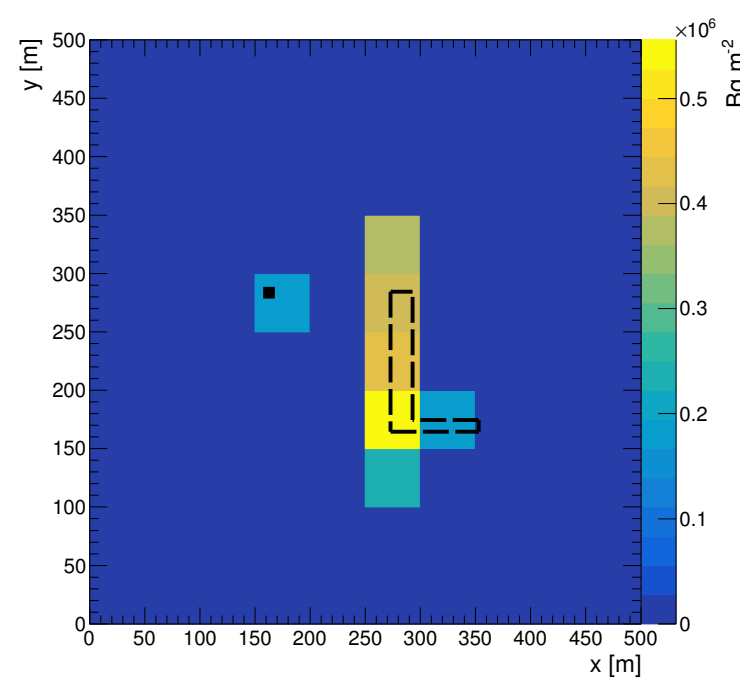

(a)

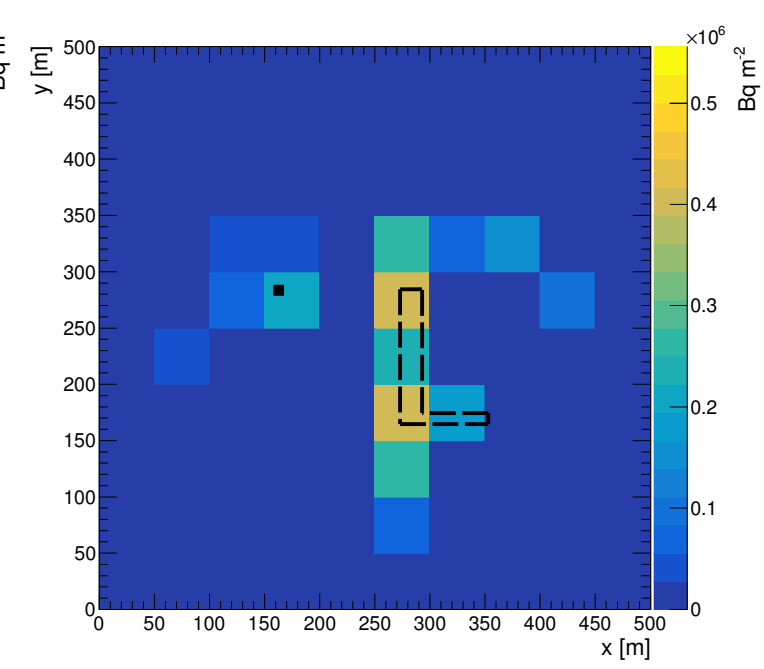

(b)

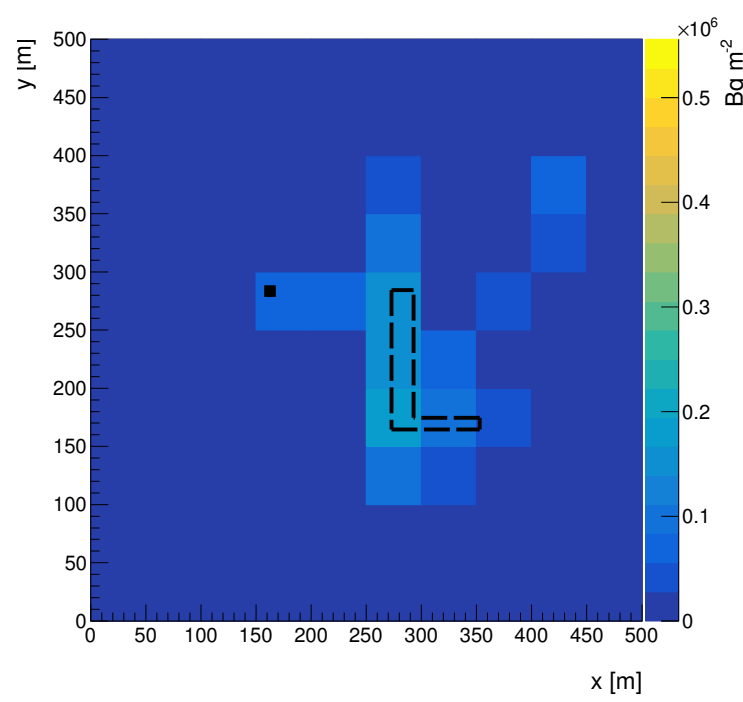

(c)

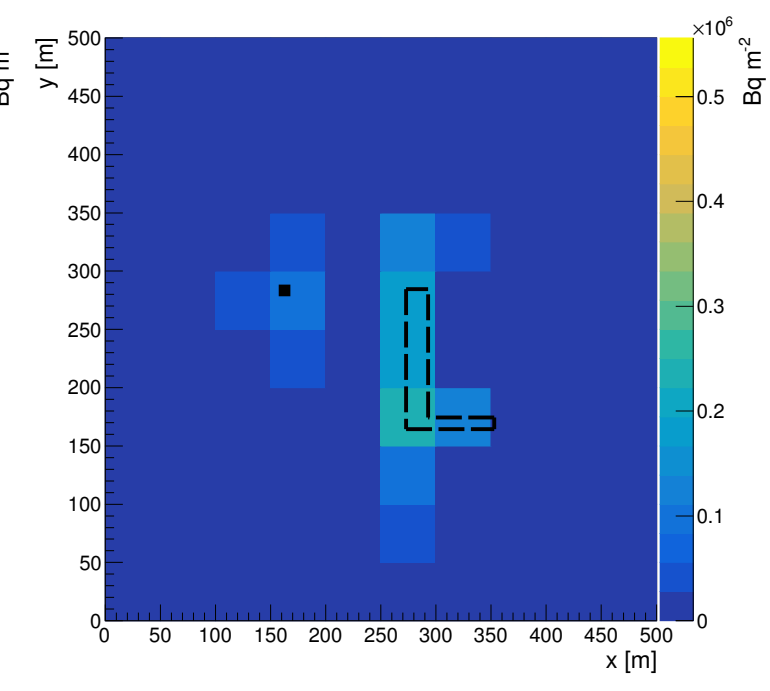

(d)

Figure 6.31: Unfolded distribution of La-140 in the Suffield experiment. a) Estimated La-140 distribution. b) Systematic uncertainty estimate. c) Statistical uncertainty estimate generated by repeated $M C$ simulation of the distribution in figure $a$. d) Statistical uncertainty estimate generated by bootstrap resampling of the experimentally measured data. All figures are presented in coordinates relative to the southwestern border of the restricted access zone and on the same colour scale. Dashed black lines denote the expected distribution of La-140, and the closed black square marks the placement of the sprayer $U G V$.

- Yaw angle of detector: The response data used in calculating $\tilde{f}$ were retained, and 12 independent unfolded reconstructions were made wherein each 
of the dwell points individually had $\pm \sigma$ added to their yaw angle during the tomographic reconstruction process, where the values of $\sigma$ used are those presented in table 6.5 .

- Pitch angle of detector: 12 independent unfolded reconstructions were performed using the same response data as were used to obtain $\tilde{f}$, individually shifting the apparent horizon by $\pm 2^{\circ}$ at each dwell point during the tomographic back-projection process.

- Detector interplane gap: The response data used to obtain $\tilde{f}$ were reused, and an uncertainty of $\pm 0.5 \mathrm{~cm}$ was assumed on the nominal value of $20 \mathrm{~cm}$. Two independent unfolded reconstructions were made wherein all detectors had their interplane gap adjusted by either $\pm 0.5 \mathrm{~cm}$ and new values of the complementary cone-axis polar angle $\alpha^{\prime}$ for each Compton event calculated using equations 4.2 and 4.3 before tomographic back-projection.

- Detector height: To estimate the systematic uncertainty associated with the assumption that the source was in the same plane as the detector, new MC response template data were generated with all six dwell points simultaneously at a height of $2.5 \mathrm{~m}$ from the plane containing the source. An unfolded reconstruction was then made using this response data.

- Air density: The systematic uncertainty due to air density was estimated by generation of new MC response template data using an environment made with air at $92 \mathrm{kPa}$ and $0{ }^{\circ} \mathrm{C}$; a choice was made to change only the air pressure in this calculation since the air pressure dominated the potential change in air density.

- Energy scale: The systematic uncertainty due to changes in the energy scale was estimated by performing 12 independent unfolded reconstructions, each time changing the scaling factor applied to the energy scale by $\pm 1 \%$.

- Background subtraction: To estimate the systematic uncertainty incurred by the background subtraction process, a result was generated using a separate background subtraction technique from the one presented in section 4.3. Assuming that the rate of NORM background contribution was consistent between all dwell points, the NORM background data were scaled by the ratio of the acquisition time at each dwell point to the acquisition time for the NORM 
background dataset. The resulting scaled NORM background data were then subtracted from the La-140 + NORM background data to obtain a backgroundsubtracted La-140 dataset, and these data were passed through the unfolding procedure and compared to $\tilde{f}$.

As outlined above, the MC response data used to obtain $\tilde{f}$ were retained when estimating the systematic uncertainties associated with the yaw angle, the pitch angle, and the interplane gap of the detector. This approach expedites the estimation of systematic uncertainties, and the assumption made is valid as it is expected that the response of the detector would not change significantly within the parameters that were varied. Not investigated were the contributions of systematic uncertainty due to any roll of the detector about its central axis and due to the uncertainty on the position of the detector. This will be discussed and justified later in this section.

The resulting estimate of the systematic uncertainties is shown in figure $6.31 \mathrm{~b}$. At the location of the sprayer UGV, the systematic uncertainty is just below the reconstructed concentration of La-140, suggesting that significant concentration of La-140 remains at the $1 \sigma$ level. This is similarly true everywhere within the L-polygon, including the small arm. Comparison of figure 6.31b to the statistical uncertainty estimates in figures $6.31 \mathrm{c}$ and $6.31 \mathrm{~d}$ shows that the systematic uncertainties dominate over statistical uncertainties. Figure 6.32 shows the individual components that contributed to the overall systematic uncertainty, all presented on the same colour scale as $\tilde{f}$ shown in figure $6.31 \mathrm{a}$. The components of systematic uncertainty due to the pitch and yaw angles of the detector and due to the energy scale correction are seen to dominate. Therefore, future experiments should seek to improve the ability to measure the orientation of the detector and to stabilize the energy scale. Since the Suffield experiment was conducted in 2018, the 3x3-module SCoTSS detector has been investigated with new technologies capable of providing better orientation estimates of the detector, which would significantly reduce the overall systematic uncertainty of an unfolded result in a future application. As shown in chapter 5, one such technology is LIDAR. An inertial measurement unit (IMU) capable of providing orientation estimates is also installed, and can be paired with LIDAR data, or used independently, to further improve the orientation estimate. To stabilize the energy scale, thermal effects may be moderated by lining the detector with an insulating jacket, or automatic gain-stabilization techniques may be investigated and applied. 


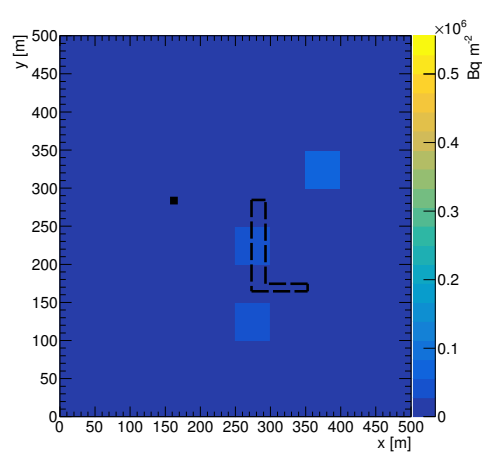

(a)

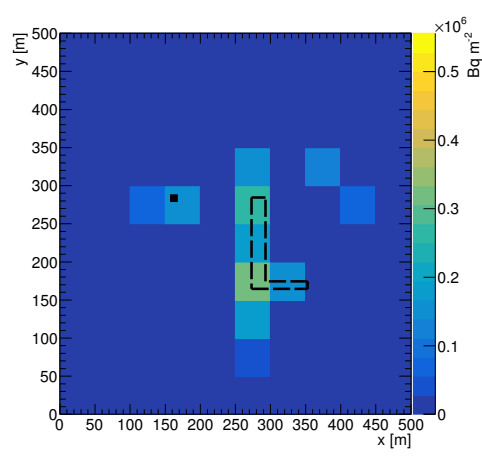

(d)

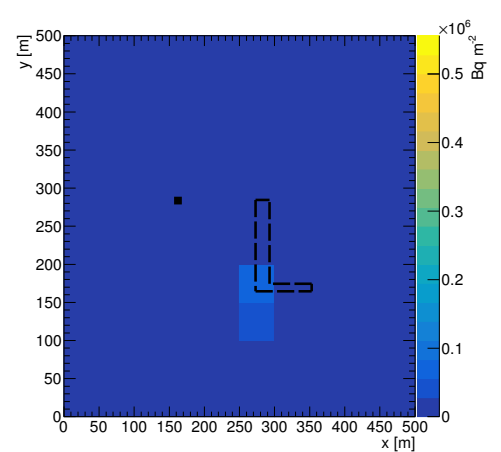

(b)

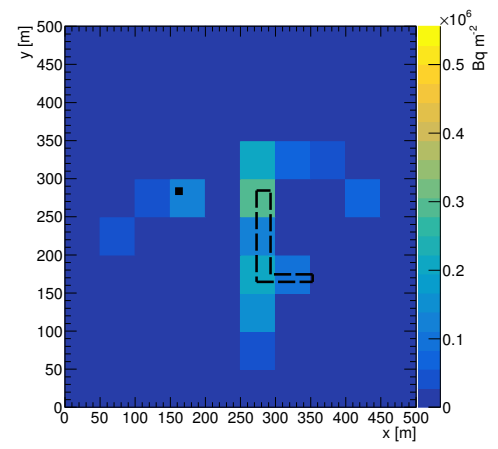

(e)

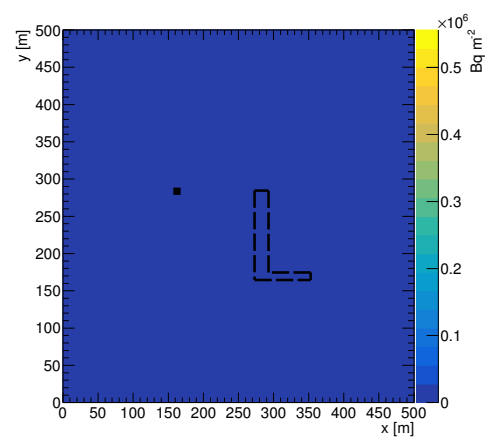

(g)

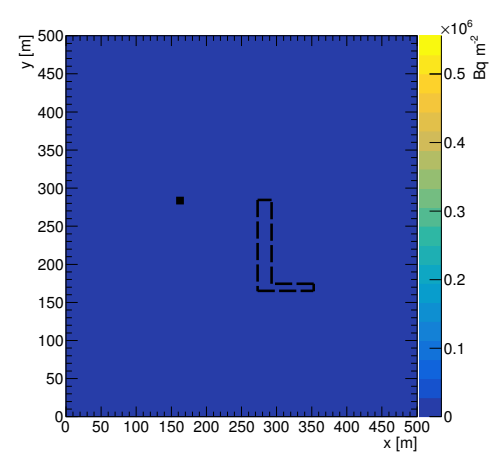

(c)

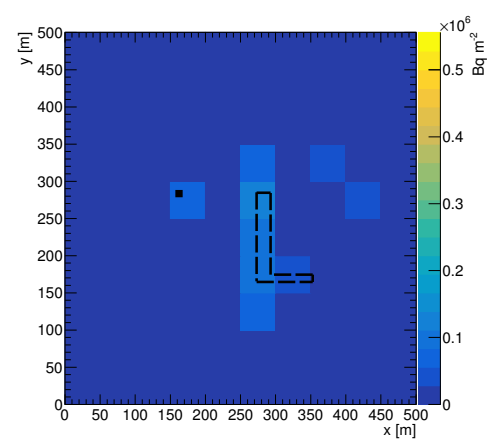

$(f)$

Figure 6.32: Components of systematic uncertainty on unfolded experimental La140 distribution. a) Effect of air density. b) Effect of detector height. c) Effect of detector inter-plane gap. d) Effect of detector pitch angle. e) Effect of detector yaw angle. f) Effect of detector energy scale. g) Effect of background subtraction. All figures are presented in the same coordinates and on the same colour scale as figure 6.31a.

Table 6.6 shows the total activity reconstructed in $\tilde{f}$, along with estimates of the systematic and statistical uncertainties. The final estimate of the total activity present in the restricted-access zone was $(6.1 \pm 1.0[$ stat.] $\pm 2.0[$ sys.] $)$ GBq for both the 
repeated MC simulation and the bootstrapping methods of statistical uncertainty estimation. Comparison of the total activity recovered by the spatial-unfolding method to the expected value of $35 \mathrm{GBq}$ yields of Z-score of -13.1 and a two-sided P-value of 0.00 , and the total recovered activity is therefore in disagreement with the expected value at a significance level of 0.01 . It is believed that snow and soil attenuators not included in the MC simulation of the detector response account for the vast underestimation of the La-140 activity, in addition to the effects mentioned previously in section 5.2.3. The possible effects of snow and soil is explored in the following section.

Table 6.6: Total estimated activity and associated uncertainties from spatial unfolding of La-140 distribution in the Suffield experiment.

\begin{tabular}{ll}
\hline Total activity & $6.1 \mathrm{GBq}$ \\
\hline Total systematic uncertainty & $2.0 \mathrm{GBq}$ \\
Air density & $0.2 \mathrm{GBq}$ \\
Detector height & $0.2 \mathrm{GBq}$ \\
Inter-plane gap & $0.1 \mathrm{GBq}$ \\
Detector pitch & $1.4 \mathrm{GBq}$ \\
Detector yaw & $1.2 \mathrm{GBq}$ \\
Energy scale & $0.6 \mathrm{GBq}$ \\
Background subtraction & $0.1 \mathrm{GBq}$ \\
Total statistical uncertainty from repeated MC simulation & $1.0 \mathrm{GBq}$ \\
Total statistical uncertainty from bootstrap estimation & $1.0 \mathrm{GBq}$ \\
\hline
\end{tabular}

The roll angle of the detector is expected to contribute very little uncertainty, since the symmetry of the detector planes about the central axis of the detector make it equivalently sensitive to incoming gamma rays no matter what the roll angle is about its central axis. A significant roll angle would cause the Compton gamma image to appear "rolled", maintaining the measured count rate but rotating the reconstructed image of La-140 relative to the true horizon and thus affecting the tomographic reconstruction and unfolding process. Inspection of the field-of-view images shown in figures 6.10 to 6.15 shows that any roll angle of the detector is small, and within the resolution of the detector specified by $\sigma_{\mathrm{ARM}}$ is insignificant.

The systematic uncertainty contributed by the position of the detector was similarly negligible. Although the GNSS-measured positions of each dwell point had 
an uncertainty on the order of $3 \mathrm{~m}$, this is a small perturbation compared to the several hundred meters over which the tomographic reconstruction was made. The uncertainty on the GNSS-measured position of each dwell point would allow for a "circle of uncertainty" about the detector at each dwell point within which the detector position may have fluctuated, with the most extreme cases corresponding to fluctuations parallel to the border of the restricted-access zone or directly towards or away from the source. The detector was no closer than $175 \mathrm{~m}$ from the source, and assuming a point source $175 \mathrm{~m}$ from the detector results in an inverse-square effect of $3-4 \%$ if the detector is brought $3 \mathrm{~m}$ closer or further from the source along the line connecting their positions. A more representative source-detector distance for all dwell points would be approximately $250 \mathrm{~m}$, for which the inverse-square law results in an effect of approximately $2 \%$. For $3 \mathrm{~m}$ translations of the detector parallel to the perimeter of the restricted-access zone, the relative angular displacement of a point source at $250 \mathrm{~m}$ distance is less than $1^{\circ}$. In either case, these effects are dominated by the systematic uncertainties introduced by the energy scale correction and by the yaw and pitch angle estimates. Furthermore, the effects discussed above represent the most extreme values that would be ascribed to the positioning uncertainty of the detector at each dwell point, and it is unlikely that the fluctuation in measured position would have been perfectly parallel to the border of the restricted-access zone or directly towards or away from the source.

Unlike the experimental measurements of Cs-137 that were discussed in chapter 5, the $1.596 \mathrm{MeV}$ gamma-ray emission selected for imaging of La-140 is not the highest-energy emission. Table 6.2 shows that an average of 4.6 photons with energies larger than $1.596 \mathrm{MeV}$ are emitted for every 100 decays of La-140. It is possible that some of these higher-energy gamma rays may have scattered from the soil or from the air and contributed a "false-positive" signal consistent with the selection criteria for the $1.596 \mathrm{MeV}$ emission, an effect which would not be captured by the simulated representation of the $3 \times 3$-module SCoTSS detector due to the use of a collimated source model and the omission of ground material from the simulated environment. This presents a negligible effect, however, due both to the relatively low proportion of higher-energy photons emitted by La-140 and to the small solid angle of the detector within which these scattered photons must have intersected while still having an energy consistent with the selection criteria for the $1.596 \mathrm{MeV}$ emission. Furthermore, 
it was demonstrated in section 6.1.4 that the $\mathrm{MC}$ representation of the experimental scenario describes the structure of the experimental data well, supporting the conjecture that the collimated-source simulations and omission of environmental terrain in the simulated geometry was reasonably able to model the physics of the gamma-ray radiation that managed to reach the detector. 


\section{Effect of environmental terrain not included in MC response}

The total activity reconstructed by the spatial-unfolding method agrees at a significance level of 0.01 with the activity estimate produced by the point-model estimate presented in section 6.2.2, yielding a Z-score of 0.31 and a two-sided P-value of 0.76. However, both values are in disagreement with the reported $35 \mathrm{GBq}$ of La-140 that was deposited. As discussed in section 5.2.3, factors not included in the simulated representation of the detector are likely the cause of the disagreement between experiment and MC. While it is true that the emission rate estimate for the $662 \mathrm{keV}$ point source presented in section 5.2 .3 was underestimated by approximately $17 \%$, the activity of the La-140 presented here has been underestimated by a much greater amount, $83 \%$ or a factor of 5.7. In addition to the discrepancies between the simulated and real detector discussed in section 5.2.3, it is very likely that this larger discrepancy is due to intervening snow and soil present during the experimental trial in Suffield, which contributed to attenuation of the La-140 signal which was not accounted for in the MC response templates used in the spatial-unfolding process.

To investigate the possible effect of snow and soil attenuators, analytic calculations were made to determine the apparent activity which would remain following attenuation of the $1.596 \mathrm{MeV}$ photons by varying amounts of snow and soil (attenuation by intervening air is accounted for in the response templates). The composition of the soil at the Suffield experimental site was unknown, so its composition was assumed to be the same as a general soil model utilized by the International Commission on Radiation Units and Measurements (ICRU). ${ }^{39}$ Table 6.7 lists the assumed composition of the soil. Previous geological surveys of the DRDC Suffield site de-

Table 6.7: Composition of the soil assumed for attenuation calculations in the Suffield experiment.

\begin{tabular}{c|c} 
Element & Percentage by mass \\
\hline $\mathrm{H}$ & 2.2 \\
$\mathrm{O}$ & 57.5 \\
$\mathrm{Al}$ & 8.5 \\
$\mathrm{Si}$ & 26.2 \\
$\mathrm{Fe}$ & 5.6
\end{tabular}

termined that the average soil density was approximately $1.6 \mathrm{~g} / \mathrm{cm}^{3}$ when dry and 
approximately $1.8 \mathrm{~g} / \mathrm{cm}^{3}$ when at typical moisture levels. ${ }^{79}$ Two extreme circumstances were investigated. In the first, settled snow with a density of $0.3 \mathrm{~g} / \mathrm{cm}^{3}$ was assumed $^{80}$ and paired with soil at a density of $1.6 \mathrm{~g} / \mathrm{cm}^{3}$. In the second, wind-packed snow with a density of $0.4 \mathrm{~g} / \mathrm{cm}^{3}$ was assumed ${ }^{80}$ and paired with soil at a density of $1.8 \mathrm{~g} / \mathrm{cm}^{3}$. These two circumstances represent the extreme limits of the effects of snow and soil which may have been encountered in the experimental conditions; the second of the two scenarios is likely closer to the truth. Mass-attenuation data for $1.596 \mathrm{MeV}$ photons in snow were obtained using the NIST XCOM database ${ }^{29}$ and were taken from the ICRU publication for the soil composition. ${ }^{39}$ Assuming combinations of differing thicknesses of snow and soil, the rate of $1.596 \mathrm{MeV}$ photons emitted from a $35 \mathrm{GBq}$ source of La-140 that escape from the snow and soil was calculated using equation 2.10 and then related back to an apparent activity of La-140 using the branching ratio of the $1.596 \mathrm{MeV}$ emission given in table 6.2.

Snow banks and light snow fall or frost may have obstructed the path of the gamma rays. Signal loss in soil likely occurred through migration of the aqueous La140 solution into the soil as well as through undulations in the terrain that partially shielded the La-140 from the detector (e.g. due to tire tracks in the soil made by the UGV sprayer). Figure 6.33 shows contour plots indicating the apparent activity of La-140 remaining after attenuation due to different path lengths in snow and soil for both of the scenarios outlined above, where the contours are in units of GBq. A contour has specifically been plotted to indicate the combinations of snow and soil for which an apparent activity of $6.1 \mathrm{GBq}$ of La-140 is detectable. By way of example, approximately $10 \mathrm{~cm}$ of intervening soil and $55 \mathrm{~cm}$ of intervening snow are required to reduce the measurable signal to $6.1 \mathrm{GBq}$ assuming snow at a density of $0.3 \mathrm{~g} / \mathrm{cm}^{3}$ and soil at a density of $1.6 \mathrm{~g} / \mathrm{cm}^{3}$, and approximately $10 \mathrm{~cm}$ of intervening soil and $37 \mathrm{~cm}$ of intervening snow are required for the scenario in which the snow had a density of $0.4 \mathrm{~g} / \mathrm{cm}^{3}$ and the soil had a density of $1.8 \mathrm{~g} / \mathrm{cm}^{3}$. The environmental conditions in March 2018 were such that these amounts of intervening snow and soil could well have occurred to reduce the measurable signal to the equivalent of approximately $6.1 \mathrm{GBq}$.

To demonstrate the potential of the method on a similar measurement geometry and in the absence of external attenuators such as snow and soil, an L-shaped distribution of La-140 with a total activity of $35 \mathrm{GBq}$ and a uniform surface activ- 
ity concentration was simulated in the experimental geometry with statistics similar to that of the experimental data. Figure 6.34 shows the results of unfolding these synthetic data, along with statistical uncertainty estimates generated by repeating the simulation and unfolding 25 times. The unfolded result shown in figure 6.34a demonstrates good localization of the source, and the errant region of activity between the arms of the L-polygon is consistent with $0 \mathrm{~Bq} / \mathrm{m}^{2}$ within the uncertainties presented in figure $6.34 \mathrm{~b}$. The total activity contained in the reconstructed estimate was $(33.1 \pm 4.0) \mathrm{GBq}$, yielding a Z-score of -0.48 when compared to the simulated activity of $35 \mathrm{GBq}$. This Z-score results in a two-sided P-value of 0.63 , and the total reconstructed activity is therefore in agreement with the expected value at a significance level of 0.01 . Note also that this result demonstrates the ability of the spatial-unfolding method to recover the total activity of a complicated source shape, even when the size of the response templates used is not commensurate with the size of the underlying source distribution. 


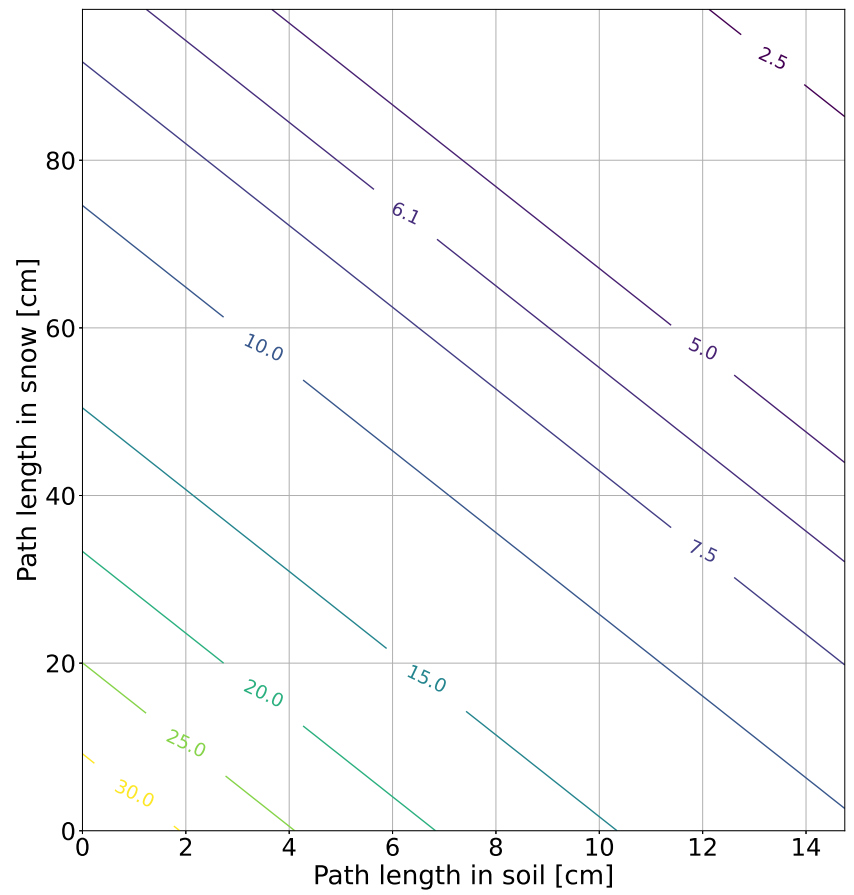

(a)

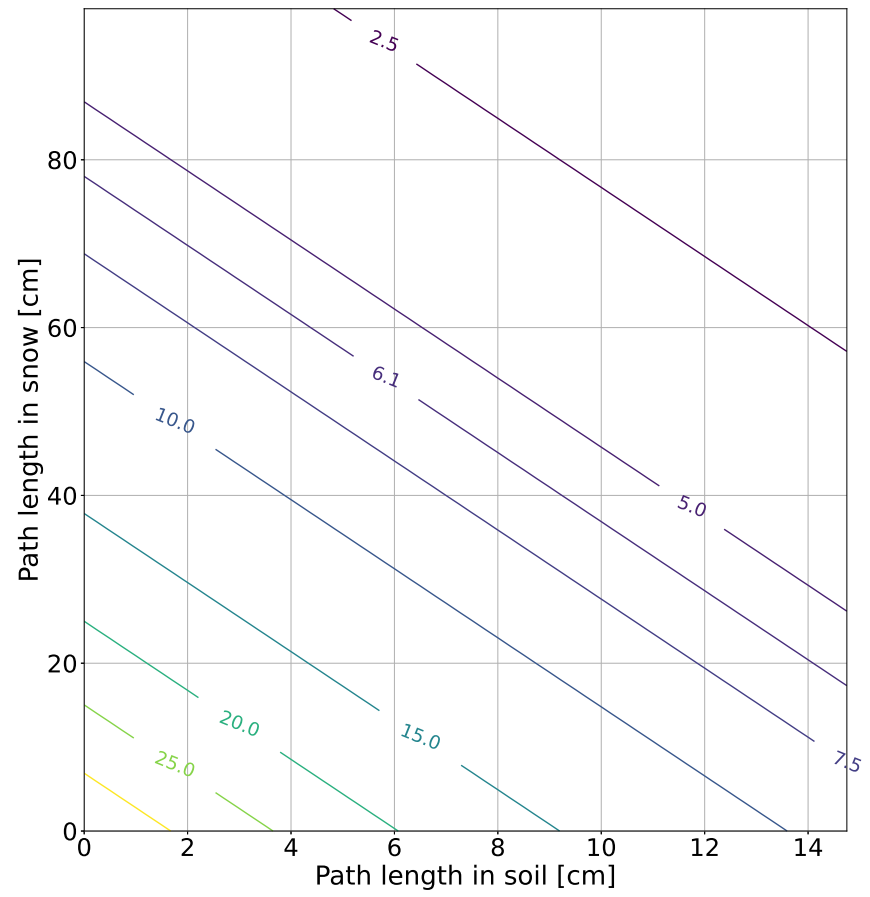

(b)

Figure 6.33: Apparent La-140 activity in the Suffield experiment following attenuation due to snow and soil. a) Effects of snow with a density of $0.3 \mathrm{~g} / \mathrm{cm}^{2}$ and soil with a density of $1.6 \mathrm{~g} / \mathrm{cm}^{2}$. b) Effects of snow with a density of $0.4 \mathrm{~g} / \mathrm{cm}^{2}$ and soil with a density of $1.8 \mathrm{~g} / \mathrm{cm}^{2}$. The units of the contours are in $G B q$. 


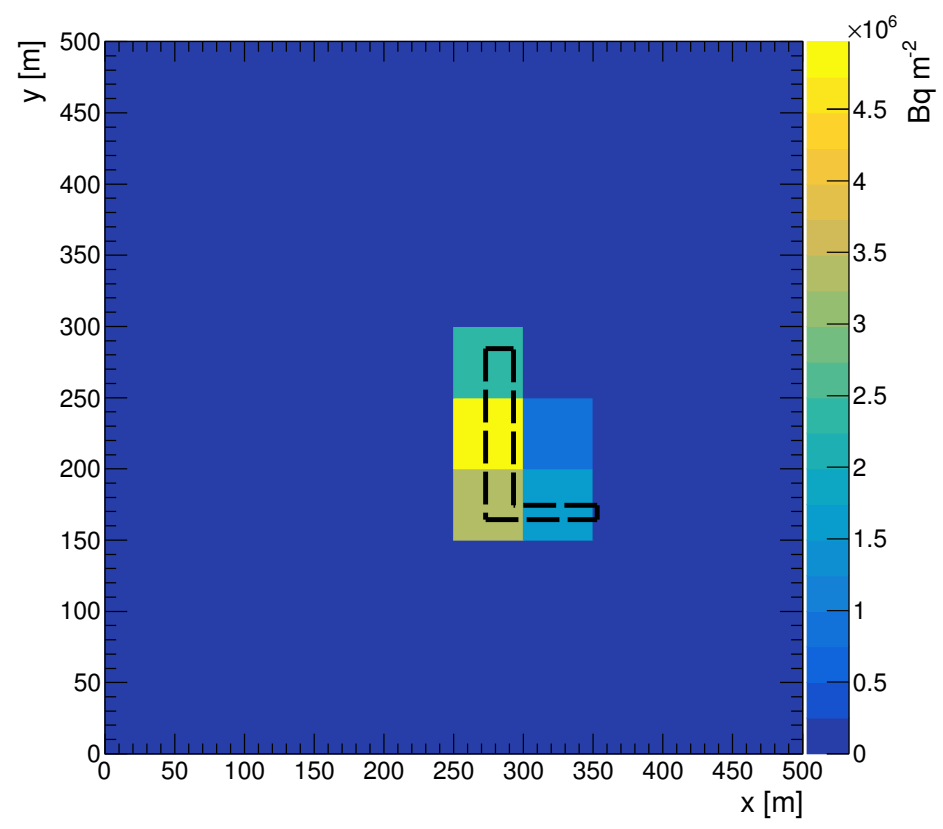

(a)

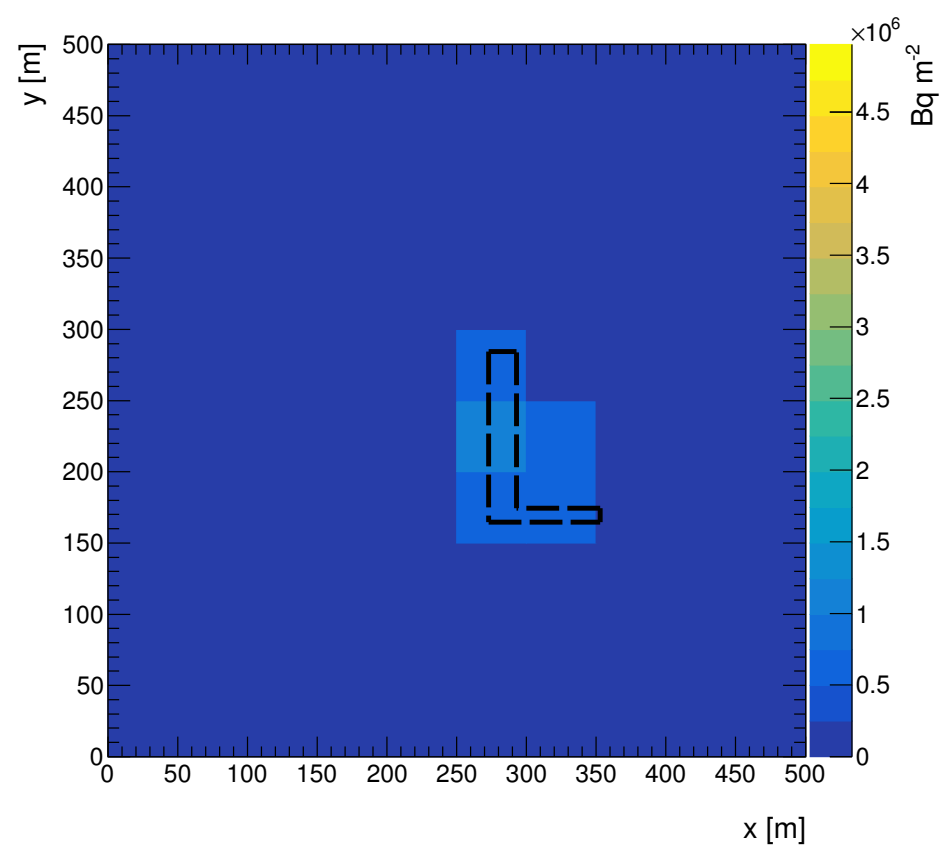

(b)

Figure 6.34: Unfolded distribution of synthetic La-140 L-shaped distribution with uniform surface activity concentration. a) Estimated La-140 distribution. b) Statistical uncertainty estimate. Figures are presented in coordinates relative to the southwestern border of the restricted access zone and on the same colour scale. Dashed black lines denote the expected distribution of La-140. 


\subsection{Summary}

The experimental trial described in this chapter was the first time that the $3 \times 3$-module SCoTSS detector technology was deployed in realistic outdoor operational conditions, and the technology performed very well. The tomographic reconstruction of the experimental data presented in section 6.2.1 completed in mere minutes, and places a region of high reconstructed intensity in a location consistent with the large arm of the expected La-140 distribution. There is little evidence of reconstructed intensity in the small arm of the L-polygon. However, this is not entirely unexpected as most of the La-140 is placed in the large arm. Thus tomographic reconstruction remains useful even for a dataset with low statistics and acquired in real-world operational conditions, which supports hypotheses 1 and 2 listed in section 1.2.

The point-model fit demonstrates reasonable localization of the experimental La140 source, despite the spatially-extended nature of the La-140 distribution. The $7 \mathrm{GBq}$ of activity reconstructed by the fit underestimates the expected value of 35 GBq. However, when the method is applied to synthetic data of a spatially extended La-140 source, it is able to reproduce the total activity of that source. Furthermore, the activity estimated by the point-model fit agrees with the total activity present in the unfolded estimate of the La-140 distribution, suggesting that there is an effect not captured by the response simulations that is present in the real-world experiment. The ability of the method to locate a real-world extended La-140 source, and the ability of the method to locate and reconstruct the activity of a synthetic extended source, lends support to hypothesis number 3 listed in section 1.2.

Finally, the spatial-unfolding method reproduces the shape of the expected distribution of La-140 reasonably well, despite the large measurement distances involved, the large systematic uncertainties incurred by estimating the direction in which the detector faced, the small sample of statistics collected, and the drift in the energy scale. The ability of the method to correct for the spoking artefacts incurred by the tomographic reconstruction results in most of the restricted-access zone being cleared of suspected La-140, a result which is of prime importance for operational efforts. The reconstructed total activity of $6.1 \mathrm{GBq}$ does not agree with the expected value of $35 \mathrm{GBq}$, but does agree with the point-model estimate. It is thought that signal attenuation due to absorption of La-140 into the soil, as well as due to intervening 
snow, is to blame for the large underestimation, as these factors are not included in the simulated response of the detector. Demonstration of the method on a synthetic L-polygon La-140 source reveals that in the absence of external attenuators, the method is able to recover the total expected activity. These results lend partial support to hypothesis number 4 listed in section 1.2 . 


\section{Chapter 7}

\section{Conclusions and future work}

\subsection{Conclusion}

Compton gamma imaging is a technology well-suited for gamma spectroscopy and source-localization applications for survey teams operating in the space of radiological safety and security. Using the kinematics of Compton scattering, gamma-ray sources can be accurately located without the need for extra mechanical collimation. In this thesis, a description of the development and testing of tomographic reconstruction methods for use in radiological safety and security applications has been presented, using the novel SCoTSS detector technology as a measurement tool. These methodologies were demonstrated using two experiments. One experiment consisted of laboratory measurements of a calibrated Cs-137 point source with a known surface emission rate of $662 \mathrm{keV}$ photons. A second experiment took place wherein a spatially-extended La-140 source was measured in realistic outdoor operational conditions. This was the first time that the SCoTSS detector technology had been deployed in such conditions.

For use in near real time during ongoing radiological safety and security applications, basic tomographic reconstructions have been investigated and shown to provide qualitatively sound source localization abilities. When measuring a point source in a controlled laboratory setting, the basic tomographic reconstruction indicates a localized region of high intensity at the known location of the source. When measuring a spatially-extended source in realistic outdoor operational conditions, the tomogram shows an extended region of high intensity consistent with the known placement of 
the source. The tomograms took only minutes to reconstruct on a laptop computer. The tomographic reconstruction methodology therefore works well in measurement conditions that may be encountered by operators in radiological safety and security operations, and the short reconstruction times further motivate the use of the method as a tool to assist in guiding operational efforts in near real time.

To estimate the strength and an effective position of a gamma-ray source during an ongoing operation, a method has been developed whereby a point-source model is assumed for the source distribution and a $\chi^{2}$ fit is performed on the measured counts using a simulated detector response model to estimate the location and strength of the hypothesized source. When applied to synthetic data generated by MC simulation, this method demonstrates excellent ability to locate a point source and estimate its activity. Additionally, application of this method to a spatially-extended synthetic source generated by MC simulation shows that the hypothesized point-source model is able to recover the activity even when the underlying distribution of radioactive materials is inconsistent with a point source. Spatial uncertainty contours on the location of the point-model fit demonstrate sensitivity to the underlying spatiallyextended source shape. When applied to experimentally measured data, the ability of the point-response model to locate the position of both a point source and a spatiallyextended source remains strong. However, the source activities are underestimated. The estimates of source activities produced by the point-model fit agree with the total activity contained in estimates produced by an advanced spatial-unfolding method, and the disagreement with the expected source activities is ascribed to factors not included in the simulations used to model the response of the detector.

Finally, a spatial-unfolding method has been developed that allows for quantitative estimation of radioactivity distributions, including spatially-extended source distributions. The method functions by simulating spatially-localized sources of radioactivity and passing them through the tomographic measurement and reconstruction process in order to provide spatial response templates. These spatial response templates are then fit to a tomographic reconstruction of the data in order to determine the amount of radioactive material at each location in the image space. The method is able to locate synthetic sources generated in MC simulation, as well as to estimate their activity. When applied to experimentally measured data, the method demonstrates excellent source-localization abilities, but underestimates the expected 
source activities. However, the underestimation is attributable to factors not included in the simulations used to generate the spatial response templates.

The hypotheses for this thesis were first presented in section 1.2. The SCoTSS detector technology performed well in realistic operational conditions. The detector was transported on the roof of a ground vehicle and was consequently subjected to bumps and vibrations. At one point, bolts holding the absorber plane shook loose, causing the absorber plane to fall out of place. However, repairs were easily accomplished at the experimental site and the detector was able to resume accurate gamma-ray measurements successfully. Throughout all of this, the detector was operated outdoors in late-winter conditions. All of this lends support to hypothesis number 1.

Constructing a tomogram by combining Compton gamma imaging measurements taken at different vantage points locates the underlying radioactive materials accurately. This is true for both point sources and spatially-extended sources. Furthermore, generation of the tomogram took only minutes on a laptop computer. These results support hypothesis number 2 .

By parametrizing the response of the detector to a simulated point-source model, a $\chi^{2}$ fit is able to recover the activity and location of synthetic sources, even when the source is spatially-extended. The method is similarly able to locate experimentallymeasured sources, but the activities are underestimated. Hypothesis number 3 cannot be rejected, because the simulated model of the detector does not include all of the effects of the real-world detector.

Finally, a spatial-unfolding method has been developed that demonstrates excellent ability to locate synthetic sources generated by MC simulation and to estimate their activity. The spatial-unfolding method continues to demonstrate excellent source-localization ability with experimental data, even when underestimating the source activity. These results, taken together, lend partial support to hypothesis number 4.

\section{$7.2 \quad$ Future work}

Development of a more accurate MC simulation of the 3x3-module SCoTSS detector is underway. This will improve the agreement between simulated and experimental 
data, improving the quality of the point-model fit method and the spatial-unfolding method. Possible explanations being investigated for the discrepancy include missing or poorly-modelled dead materials, trigger threshold effects, electronic noise, and overlapping events.

In future applications, the simulated environment used in the response simulations may also be developed in more detail to improve the agreement between MC simulated detector response and the experimental measurement. A portable electronic barometer, thermometer, and hygrometer may be used to provide estimates of the air temperature, pressure, and humidity at the location of the detector during an operational deployment, providing better estimates of the air density for use in the simulated environment. Soil samples may be acquired at the location of the detector during measurement and used to estimate the quality of the soil if a detailed simulation requires implementation of soil material, and high-resolution optical photographs may be acquired by the operator for use in estimating the shape of the terrain. In principle, a full simulation of the experimental environment would allow for accurate characterization of radioactive distributions, assuming that there is sufficient computational power available to run the simulations. To additionally estimate the effects of environmental attenuation in experimental data, a second dataset at each dwell point may be acquired with the detector placed at a larger height.

Acquiring more data in future exercises should improve the reconstruction abilities. Longer acquisition times at each dwell point can be taken, and the number of dwell points used overall may be increased. Dynamic imaging may also be investigated, allowing for the collection of data while the detector is in motion around the perimeter of a restricted-access zone. Finally, the simultaneous use of multiple gamma-ray emission energies for imaging may be investigated.

To better represent the NORM background, multiple measurements of the NORM background signal may be acquired. Ideally, a NORM background measurement would be acquired at the location of each dwell point. This may not be possible in a real-world application, however, and an alternative may be to acquire NORM background measurements in more than one region at a distance from the contaminated zone to obtain a better average proxy for the NORM background.

Researchers at the NRC and NRCan have already begun to investigate LIDAR 
and IMU technologies for use with Compton gamma imagers. The results presented in chapter 5 for a point source measured in a laboratory made use of detector orientation estimates provided by LIDAR measurements, but LIDAR technology was not available for the outdoor extended-source experiment presented in chapter 6. Estimates of the detector orientation were much more accurate with LIDAR measurements. Application of LIDAR to future experiments or operational exercises should considerably lower the systematic uncertainty on future unfolded distribution results, since the orientation of the detector was found to be a dominating source of systematic uncertainty in terms of both the amount of radioactive material that was reconstructed as well as in its placement.

The tomographic reconstruction and point-model fit methodologies are fast and may be applied during operations to assist in guiding efforts in near real time. For example, the tomographic reconstruction may guide operators to regions inside of a measured space that they should focus on, and the activity estimate provided by the point-model fit may be used to inform decisions related to factors such as radiation safety. These methods may be generalized to three dimensions with little change to the underlying theory.

The spatial-unfolding methodology developed and presented in this thesis can similarly be generalized to three dimensions. Future work will investigate the utility of the method in such an application. A multi-scale fitting approach, wherein a fit is first made with coarse response templates and regions with high levels of reconstructed activity are then re-fit with smaller response templates, may be developed to expedite the fitting process. This would prove especially useful for three-dimensional applications. The method may then be applied to analyse data acquired during radiological safety and security operations, allowing researchers to better understand the distribution of the radioactive materials that were measured. 


\section{Appendix A}

\section{Relationship between attenuation coefficient and atomic cross section}

Consider a particle that is perpendicularly incident on a slab of finite thickness $\mathrm{d} x$ and surface area $a$ with density $\rho$ and that is made from a single atomic species with atomic number $Z$ and molar mass $M_{A}$. The atomic cross section for an interaction process $i$ is ${ }_{\mathrm{a}} \sigma_{i}$. For very thin $\mathrm{d} x$ and for small ${ }_{\mathrm{a}} \sigma_{i}$, the atoms in the slab may be assumed as non-overlapping and the total effective area with respect to the $i^{\text {th }}$ interaction process, $a_{i}^{\text {eff }}$, of atoms in the slab from the point of view of the incident particle is given by the number of atoms multiplied by the atomic cross section:

$$
a_{i}^{\mathrm{eff}}=\left(\frac{\rho a N_{A} \mathrm{~d} x}{M_{A}}\right)\left({ }_{\mathrm{a}} \sigma_{i}\right)
$$

where $N_{A}$ is Avagadro's constant. The probability $\mathrm{d} P$ that the particle undergoes an interaction via the $i^{\text {th }}$ interaction process in this infinitesimal slab is given by the ratio of effective area to the actual area of the slab:

$$
\mathrm{d} P=\left(\frac{\rho N_{A}}{M_{A}}\right)\left({ }_{\mathrm{a}} \sigma_{i}\right) \mathrm{d} x .
$$

Consider now a slab of finite thickness $x$. To arrive at the standard expression for linear attenuation, the probability $P^{\prime}$ that an interaction does not occur within the slab must be calculated. This is accomplished by considering the finite slab as being composed of a series of thinner sub-slabs each with a thickness $\Delta x$, resulting in $x / \Delta x$ sub-slabs. Each sub-slab is considered as independent from the others, and 
the probability of interaction in any single sub-slab is described by equation A.2. The probability of no interaction occurring within each sub-slab is thus $(1-\mathrm{d} P)$. The overall probability of no interactions occurring anywhere within the larger slab is therefore given by the value $(1-\mathrm{d} P)$ multiplied by itself $x / \Delta x$ times for each independent sub-slab in the limit that $\Delta x \rightarrow 0$ :

$$
P^{\prime}=\lim _{\Delta x \rightarrow 0}\left(1-\frac{\rho N_{A}}{M_{A}}\left({ }_{\mathrm{a}} \sigma_{i}\right) \Delta x\right)^{x / \Delta x} .
$$

Making use of the identity $\lim _{z \rightarrow 0}(1+k z)^{m / k}=\exp (m k)$, equation A.3 reduces to

$$
P^{\prime}=\exp \left(-\frac{\rho N_{A}}{M_{A}}\left({ }_{\mathrm{a}} \sigma_{i}\right) x\right) \text {. }
$$

Equation 2.10 describing the linear attenuation process for a beam of $N_{0}$ particles perpendicularly incident on a slab of thickness $x$ is reproduced below for convenience:

$$
N=N_{0} \exp (-\mu x)
$$

where $N$ is the number of particles that traverse the slab without having undergone any interaction. Comparing equation A.4 to equation A.5 yields the following relations:

$$
\begin{aligned}
P^{\prime} & =\frac{N}{N_{0}} \\
\mu_{i} & =\frac{\rho N_{\mathrm{A}}}{M_{A}}\left({ }_{\mathrm{a}} \sigma_{i}\right)
\end{aligned}
$$

For compounds containing multiple atomic species, the effective area given by equation A.1 becomes a sum over the effective area contributions of each atomic species. Likewise, when considering multiple different interaction processes, the effective areas of each interaction process sum. Equations A.4 and A.6 are modified with the appropriate sums. 


\section{Appendix B}

\section{Significance testing with the Z-score}

The agreement between two values can be statistically assessed using the Z-score, which assumes that the difference between the values follows a standard Gaussian distribution (i.e. with a mean of zero and a standard deviation of one). ${ }^{81}$ Let $\bar{X}_{1}$ and $\bar{X}_{2}$ represent two measured values with associated uncertainties $\sigma_{\bar{X}_{1}}$ and $\sigma_{\bar{X}_{2}}$. The Z-score is given by

$$
Z=\frac{\bar{X}_{1}-\bar{X}_{2}}{\sqrt{\sigma_{\bar{X}_{1}}^{2}+\sigma_{\bar{X}_{2}}^{2}}} .
$$

The Z-score given by equation B.1 can be used in statistical tests. In this thesis, it is used in a significance test wherein the null hypothesis is "the difference between $\bar{X}_{1}$ and $\bar{X}_{2}$ is equal to zero" and the alternative hypothesis is that "the difference between $\bar{X}_{1}$ and $\bar{X}_{2}$ is not equal to zero". This is achieved through the calculation of a probability value, or P-value, which is the integral of the standard Gaussian distribution past the Z-score that was observed and is a measure of the probability that the null hypothesis would give that Z-score or a Z-score even more extreme. If the P-value is smaller than some threshold referred to as the level of significance, which is a value chosen in advance of the measurement, then the null hypothesis is rejected at that level of significance in favour of the alternative hypothesis. Values of the level of significance that are often used are 0.05 or 0.01 , but the value that is used is ultimately up to the discretion of the researcher.

There are two types of p-values which may be calculated: one-sided and twosided. The difference between the two types of P-value are illustrated in figure B.1 
using a significance value of 0.05. Figure B.1a shows a one-sided P-value, where the blue area underneath the curve encloses an area equal to 0.05. Figure B.1b shows a two-sided P-value, where each of the blue areas underneath the curve enclose an area of 0.025 for a total of 0.05 . For the purposes of testing the null hypothesis versus the alternative hypothesis as stated above, a two-sided P-value is appropriate. This is because it is a test of whether or not $\bar{X}_{1}-\bar{X}_{2}=0$, and the difference may be positive or negative.

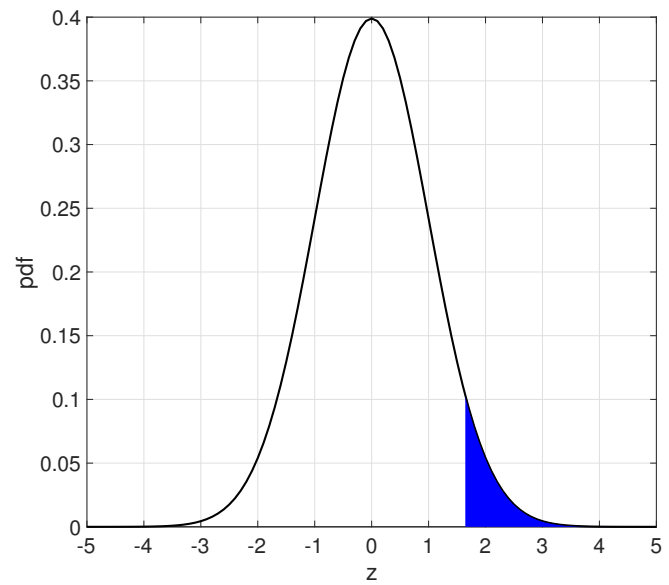

(a)

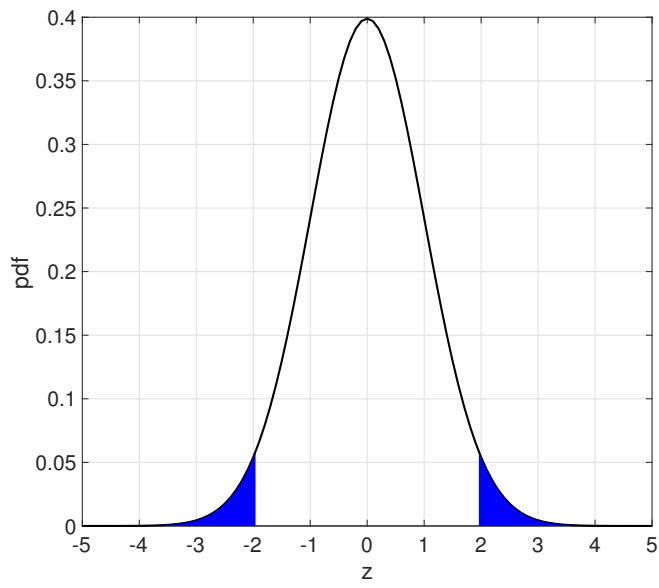

(b)

Figure B.1: Illustration of one-sided vs two-sided P-value at a significance level of 0.05. a) One-sided P-value. The blue area represents an area equal to 0.05. b) Two-sided P-value. The blue areas each represent an area equal to 0.025. 


\section{References}

1 S. Wheatley, B. K. Sovacool, and D. Sornette, "Reassessing the safety of nuclear power," Energy Research \& Social Science, vol. 15, pp. 96-100, May 2016.

2 C. Chen, L. Sinclair, R. Fortin, M. Coyle, and C. Samson, "In-flight performance of the Advanced Radiation Detector for UAV Operations (ARDUO)," Nuclear Instruments and Methods in Physics Research Section A: Accelerators, Spectrometers, Detectors and Associated Equipment, vol. 954, p. 161609, 2020. Symposium on Radiation Measurements and Applications XVII.

3 L. E. Sinclair and R. Fortin, "Spatial deconvolution of aerial radiometric survey and its application to the fallout from a radiological dispersal device," Journal of Environmental Radioactivity, vol. 197, pp. 39-47, Feb. 2019.

4 J. Zhao and C. J. Sullivan, "Detection and parameter estimation of radioactive sources with mobile sensor networks," Radiation Physics and Chemistry, vol. 155, pp. 265-270, Feb. 2019.

5 J. Zhao, Z. Zhang, and C. J. Sullivan, "Identifying anomalous nuclear radioactive sources using Poisson kriging and mobile sensor networks," PLOS ONE, vol. 14, p. e0216131, May 2019.

6 M. Tanigaki, R. Okumura, K. Takamiya, N. Sato, H. Yoshino, and H. Yamana, "Development of a car-borne gamma-ray survey system, KURAMA," Nuclear Instruments and Methods in Physics Research Section A: Accelerators, Spectrometers, Detectors and Associated Equipment, vol. 726, pp. 162-168, Oct. 2013.

7 S. Kobayashi, T. Shinomiya, H. Kitamura, T. Ishikawa, H. Imaseki, M. Oikawa, S. Kodaira, N. Miyaushiro, Y. Takashima, and Y. Uchihori, "Radioactive contamination mapping of northeastern and eastern Japan by a car-borne survey system, Radi-Probe," Journal of Environmental Radioactivity, vol. 139, pp. 281-293, Jan. 2015.

8 B. Deb, "Iterative Estimation of Location and Trajectory of Radioactive Sources With a Networked System of Detectors," IEEE Transactions on Nuclear Science, vol. 60, pp. 1315-1326, Apr. 2013.

9 "Multi-Agency Radiation Survey and Site Investigation Manual (MARSSIM)," tech. rep., United States Environmental Protection Agency, August 2000. Text accessed online on January 15th 2021 at https://www.epa.gov/radiation/ download-marssim-manual-and-resources. 
P. Martin, O. Payton, Y. Yamashiki, D. Richards, and T. Scott, "High-resolution radiation mapping to investigate FDNPP derived contaminant migration," Journal of Environmental Radioactivity, vol. 164, pp. 26-35, Nov. 2016.

11 S. Mikami, T. Maeyama, Y. Hoshide, R. Sakamoto, S. Sato, N. Okuda, T. Sato, H. Takemiya, and K. Saito, "The air dose rate around the Fukushima Dai-ichi Nuclear Power Plant: its spatial characteristics and temporal changes until December 2012," Journal of Environmental Radioactivity, vol. 139, pp. 250-259, Jan. 2015.

12 K. Saito and Y. Onda, "Outline of the national mapping projects implemented after the Fukushima accident," Journal of Environmental Radioactivity, vol. 139, pp. 240-249, Jan. 2015.

13 S. Tsuda and K. Saito, "Spectrum-dose conversion operator of $\mathrm{NaI}(\mathrm{Tl})$ and $\mathrm{CsI}(\mathrm{Tl})$ scintillation detectors for air dose rate measurement in contaminated environments," Journal of Environmental Radioactivity, vol. 166, pp. 419-426, Jan. 2017.

14 N. Matsuda, S. Mikami, T. Sato, and K. Saito, "Measurements of air dose rates in and around houses in the Fukushima Prefecture in Japan after the Fukushima accident," Journal of Environmental Radioactivity, vol. 166, pp. 427-435, Jan. 2017.

15 Nuclear Emergency Preparedness and Response Division, Health Canada, Federal nuclear emergency plan: Part 1. 2014. OCLC: 882718142, https://central. bac-lac.gc.ca/ . item?id=H129-35-2014-eng\&op=pdf\&app=Library.

16 A. MacLeod, Development of a Compton-scatter Gamma-ray Imager. PhD thesis in physics, McGill University, 2013.

17 L. Sinclair, P. Saull, D. Hanna, H. Seywerd, A. MacLeod, and P. Boyle, "Silicon Photomultiplier-Based Compton Telescope for Safety and Security (SCoTSS)," IEEE Trans. Nucl. Sci., vol. 61, pp. 2745-2752, Oct. 2014.

18 L. E. Sinclair and P. R. B. Saull, "Compton imaging for standoff radiation detection: Report A," Tech. Rep. 7478, 2017. https://geoscan.nrcan.gc.ca/ starweb/geoscan/servlet. starweb?path=geoscan/fulle. web\&search1=R= 302775.

19 L. E. Sinclair, A. McCann, P. R. Saull, R. L. Mantifel, C. V. Ouellet, P.-L. Drouin, A. M. Macleod, B. Le Gros, I. Summerell, J. H. Hovgaard, S. Monkhouse, F. Stanescu, G. Jonkmans, and N. Murtha, "End-user experience with the SCoTSS Compton imager and directional survey spectrometer," Nuclear Instruments and Methods in Physics Research Section A: Accelerators, Spectrometers, Detectors and Associated Equipment, vol. 954, p. 161683, Feb. 2020.

20 C. G. Wahl, W. R. Kaye, W. Wang, F. Zhang, J. M. Jaworski, A. King, Y. A. Boucher, and Z. He, "The polaris-h imaging spectrometer," Nuclear Instruments 
and Methods in Physics Research Section A: Accelerators, Spectrometers, Detectors and Associated Equipment, vol. 784, pp. 377-381, 2015. Symposium on Radiation Measurements and Applications 2014 (SORMA XV).

21 A. Haefner, R. Barnowski, P. Luke, M. Amman, and K. Vetter, "Handheld realtime volumetric 3-D gamma-ray imaging," Nuclear Instruments and Methods in Physics Research Section A: Accelerators, Spectrometers, Detectors and Associated Equipment, vol. 857, pp. 42-49, June 2017.

G. Knoll, Radiation Detection and Measurement. New Jersey: Wiley, 4 ed., 2010.

"CsI(pure) Cesium Iodide Scintillation Material." https://www.crystals. saint-gobain.com/sites/imdf.crystals.com/files/documents/

csi-pure-material-data-sheet_69770.pdf, 2016. Accessed: Feb 82021.

24 K. Vetter, M. Burks, C. Cork, M. Cunningham, D. Chivers, E. Hull, T. Krings, H. Manini, L. Mihailescu, K. Nelson, D. Protic, J. Valentine, and D. Wright, "High-sensitivity Compton imaging with position-sensitive Si and Ge detectors," Nuclear Instruments and Methods in Physics Research Section A: Accelerators, Spectrometers, Detectors and Associated Equipment, vol. 579, pp. 363-366, Aug. 2007.

25 A. Sokolov, V. Gostilo, A. Loupilov, and V. Zalinkevich, "Performance improvement of $\mathrm{Si}(\mathrm{Li})$ Peltier cooled detectors," IEEE Trans. Nucl. Sci., vol. 49, pp. 24272430, Oct. 2002.

26 The Preparatory Commission for the Comprehensive Nuclear Test Ban Treaty Organization, "Comprehensive Nuclear-Test-Ban Treaty (CTBT)." Text accessed online on March 25th 2020 at https://www.ctbto.org/the-treaty/ treaty-text/.

27 M. Sakai, R. K. Parajuli, M. Kikuchi, M. Yamaguchi, Y. Nagao, N. Kawachi, K. Arakawa, and T. Nakano, "Effect of number of views on cross-sectional Compton imaging: A fundamental study with backprojection," Physica Medica, vol. 56, pp. 1-9, Dec. 2018.

28 P. Andreo, D. Burns, A. Nahum, J. Seuntjens, and F. Attix, Fundamentals of Ionizing Radiation Dosimetry. Weinheim, Germany: Wiley-VCH, 2017.

29 M. Berger, J. Hubbell, S. Seltzer, J. Chang, J. Coursey, R. Sukumar, D. Zucker, and K. Olsen, "XCOM: Photon cross sections database." http://physics .nist. gov/xcom, 2010. Accessed: Sept 142020.

30 F. Attix, Introduction to Radiological Physics and Radiation Dosimetry. Weinheim, Germany: Wiley-VCH, 2004.

31 L. E. Sinclair, D. S. Hanna, A. M. L. MacLeod, and P. R. B. Saull, "Simulations of a Scintillator Compton Gamma Imager for Safety and Security," IEEE Trans. Nucl. Sci., vol. 56, pp. 1262-1268, June 2009. 
32 "CsI( $\mathrm{Tl}), \quad \mathrm{CsI}(\mathrm{Na})$ Cesium Iodide Scintillation Material." https: //www.crystals.saint-gobain.com/sites/imdf.crystals.com/files/ documents/csitl-and-na-material-data-sheet.pdf, 2016. Accessed: Feb 8 2021.

33 N. Grassi, G. Casini, M. Frosini, G. Tobia, and T. Marchi, "PIXE characterization of $\mathrm{CsI}(\mathrm{Tl})$ scintillators used for particle detection in nuclear reactions," Nuclear Instruments and Methods in Physics Research Section B: Beam Interactions with Materials and Atoms, vol. 266, pp. 2383-2386, May 2008.

34 J. D. Valentine, W. W. Moses, S. E. Derenzo, D. K. Wehe, and G. F. Knoll, "Temperature depdendence of $\mathrm{CsI}(\mathrm{Tl})$ gamma-ray excited scintillation characteristics," Nuclear Instruments and Methods in Physics Research A, pp. 147-157, 1993.

35 M. Kim, H. Kang, H. Kim, W. Kim, H. Park, and S. Kim, "A Study of CsI(Tl) Scintillator with Optimized Conditions of Large Area Avalanche Photodiode," Journal of Nuclear Science and Technology, vol. 45, pp. 586-589, June 2008.

36 M. Moszynski, M. Kapusta, D. Wolski, M. Szawlowski, and W. Klamra, "Energy resolution of scintillation detectors readout with large area avalanche photodiodes and photomultipliers," IEEE Transactions on Nuclear Science, vol. 45, pp. 472477, June 1998.

37 "An Introduction to the Silicon Photomultiplier," tech. rep., SensL, 2011. Accessed on June 132018 at https://www.sensl.com/downloads/ds/TN\%20-\% 20Intro $\% 20$ to $\% 20 \mathrm{SPM} \% 20 \mathrm{Tech}$. pdf.

38 "C-Series SiPM Sensors: Silicon Photomultipliers (SiPM), Low-Noise, BlueSensitive," tech. rep., ON Semiconductor, 2014. Accessed on Feb 162021 at https : //www . onsemi.com/pub/Collateral/MICROC-SERIES-D . PDF.

39 I. C. on Rad. Units and Meas., Gamma ray spectrometry in the environment. No. 53 in ICRU report, Bethesda, Md., U.S.A: ICRU, 1994.

40 Canadian NORM Working Group, Canada, Health Canada, and Federal Provincial Territorial Radiation Protection Committee (Canada), Canadian guidelines for the management of naturally occurring radioactive material (NORM). 2014. OCLC: 995583354.

41 International Atomic Energy Agency, Safety Report Series, Radiation Protection and NORM Residue Management in the Production of Rare Earths from Thorium Containing Minerals. No. 68, Vienna, Austria: IAEA, 2011.

42 International Atomic Energy Agency, Technical Report Series, Airborne Gamma Ray Spectrometer Surveying. No. 323, Vienna, Austria: IAEA, 1991.

43 International Atomic Energy Agency. Nuclear Fuel Cycle and Materials Section, Guidelines for radioelement mapping using gamma ray spectrometry data. Vienna: International Atomic Energy Agency, 2003. OCLC: 703977515. 
44 Gamma-Ray Spectrum Catalogue: Ge and Si Detector Spectra. Idaho National Laboratory, 4th edition ed., March 1999.

45 R. Heath, Scintillation Spectrometry: Gamma-Ray Spectrum Catalogue, vol. 1 of 2. U.S. Atomic Energy Commission, second edition ed., Aug. 1964.

Glen Cowan, Statistical Data Analysis. Toronto: Oxford University Press, 1998.

47 F. Marshall, Reconstruction of the Spatial Distribution of Surface Activity Concentration for an In-Situ, Gamma-Ray, Truck-Borne Survey. MSc thesis in physics, Carleton University, Ottawa, Ontario, 2014.

48 L. E. Sinclair and R. Fortin, "Spatial deconvolution of aerial radiometric survey and its application to the fallout from a radiological dispersal device," Journal of Environmental Radioactivity, vol. 197, pp. 39-47, Feb. 2019.

49 L. Sinclair, P. Saull, D. Hanna, H. Seywerd, A. MacLeod, and P. Boyle, "Silicon Photomultiplier-Based Compton Telescope for Safety and Security (SCoTSS)," IEEE Trans. Nucl. Sci., vol. 61, pp. 2745-2752, Oct. 2014.

50 User Manual UM3246 DT5724 2/4 Channel 14bit 100 MS/s Digitizer. CAEN Group, Jan. 2017. Accessed online November 192019 at https://www . caen.it/ products/dt5724/.

51 I. Kawrakow, E. Mainegra-Hing, D. W. O. Rogers, F. Tessier, and B. R. B. Walters, "The EGSnrc code system: Monte Carlo simulation of electron and photon transport," 2017. Accessed online at http://nrc-cnrc.github.io/EGSnrc/ doc/pirs701-egsnrc.pdf.

52 I. Kawrakow, E. Mainegra-Hing, F. Tessier, R. Townson, and B. Walters, "Egsnrc c++ class library: EGS_CollimatedSource class reference." Text accessed online on Mar 30th, 2021 at https ://nrc-cnrc .github.io/EGSnrc/doc/pirs898/ classEGS_CollimatedSource.html.

53 G. L. Zeng, Image Reconstruction: Applications in Medical Sciences. Boston: De Gruyter, first edition ed., 2017.

54 P. C. Hansen and J. S. Jorgensen, "AIR Tools II: algebraic iterative reconstruction methods, improved implementation," Numer Algor, vol. 79, pp. 107-137, Sept. 2018.

55 L. A. Shepp and Y. Vardi, "Maximum Likelihood Reconstruction for Emission Tomography," IEEE TRANSACTIONS ON MEDICAL IMAGING, no. 2, p. 10, 1982.

56 N. Bissantz, B. A. Mair, and A. Munk, "A statistical stopping rule for MLEM reconstructions in PET," in 2008 IEEE Nuclear Science Symposium Conference Record, (Dresden, Germany), pp. 4198-4200, IEEE, Oct. 2008.

57 F. Ben Bouallgue, J. F. Crouzet, and D. Mariano-Goulart, "A heuristic statistical stopping rule for iterative reconstruction in emission tomography," Ann Nucl Med, vol. 27, pp. 84-95, Jan. 2013. 

properties of SPE IEEE Trans. Nucl. Sci., vol. 49, pp. 2062-2066, Oct. 2002.

71 P. J. Markiewicz, K. Thielemans, J. M. Schott, D. Atkinson, S. R. Arridge, B. F. Hutton, and S. Ourselin, "Rapid processing of PET list-mode data for efficient uncertainty estimation and data analysis," Phys. Med. Biol., vol. 61, pp. N322N336, July 2016.

72 R. Andrae, "Error estimation in astronomy: A guide," arXiv:1009.2755 [astro-ph, physics:physics], Oct. 2010. arXiv: 1009.2755.

H. H. Barrett, T. White, and L. C. Parra, "List-mode likelihood," J. Opt. Soc. Am. A, vol. 14, p. 2914, Nov. 1997. vol. 45, pp. 957-962, June 1998. modeling of system response in list mode EM reconstruction of Compton scatter camera images," IEEE Trans. Nucl. Sci., vol. 48, pp. 111-116, Feb. 2001. //root.cern.ch/root/htmldoc/guides/minuit2/Minuit2.pdf. of the parameter errors and correlations," Computer Physics Communications, vol. 10, pp. 343-367, Dec. 1975. CERN Program Library Long Writeup D506, Version 94.1, CERN, 1994. in AIHENP'96 Workshop, Lausane, vol. 389, pp. 81-86, 1996.

R. Fletcher, "A new approach to variable metric algorithms," The Computer Journal, vol. 13, pp. 317-322, 011970.

B. Efron, "Bootstrap Methods: Another Look at the Jackknife," The Annals of Statistics, vol. 7, pp. 1-26, Jan. 1979.

B. Efron and R. Tibshirani, An Introduction to the Bootstrap. SpringerScience+Business Media, B.V., first edition ed., 1993.

M. Ibaraki, K. Matsubara, K. Nakamura, H. Yamaguchi, and T. Kinoshita, "Bootstrap methods for estimating PET image noise: experimental validation and an application to evaluation of image reconstruction algorithms," Ann Nucl Med, vol. 28, pp. 172-182, Feb. 2014.

I. Buvat, "A non-parametric bootstrap approach for analysing the statistical M. Dahlbom, "Estimation of image noise in PET using the bootstrap method," 
73 P. Besl and N. D. McKay, "A method for registration of 3-D shapes," IEEE Trans. Pattern Anal. Mach. Intell., vol. 14, pp. 239-256, Feb. 1992.

74 L. Cheng, S. Chen, X. Liu, H. Xu, Y. Wu, M. Li, and Y. Chen, "Registration of Laser Scanning Point Clouds: A Review," Sensors, vol. 18, p. 1641, May 2018.

75 J. Bushberg, J. Seibert, E. Leidholdt Jr., and J. Boone, The Essential Physics of Medical Imaging. New York: Wolters Kluwer Health, third edition ed., 2012.

76 C. Chen, Demonstration of a new gamma spectrometer designed for radiometric surveying with an unmanned aircraft system. MSc in Earth Sciences, Carleton University, Ottawa, Ontario, Canada, 2019.

77 B. Beckman, A. R. Green, L. Sinclair, B. Fairbrother, T. Munsie, and D. White, "Robotic Dispersal Technique for 35 GBq of La-140 in an L-polygon Pattern.," Health Phys, vol. 118, pp. 448-457, Apr. 2020.

78 Laboratoire National Henri Becquerel, "Atomic and nuclear data." Text accessed online on September 10th, 2018 at http://www.lnhb.fr/nuclides/La-140_ tables.pdf.

79 J. Barchard, A. Kupper, and S. Hlady, "DRDC Suffield Soil Laboratory Program: Progress Report - Piston and Onager Sites," Jan. 2004. Accessed at https: //apps.dtic.mil/sti/pdfs/ADA436651.pdf.

80 W. Paterson, Physics of Glaciers. New York: Elsevier Science Ltd., third ed., 1994.

81 M. Spiegel, J. Schiller, and R. Alu Srinivasan, Schaum's Outlines: Probability and Statistics. Toronto: McGraw Hill, fourth ed., 2013. 